\title{
Symmetries and stabilization for sheaves of vanishing cycles
}

\author{
Christopher Brav, Vittoria Bussi, Delphine Dupont, \\ Dominic Joyce, and Balázs Szendrői, \\ with an Appendix by Jörg Schürmann
}

\begin{abstract}
We study symmetries and stabilization properties of perverse sheaves of vanishing cycles $\mathcal{P} \mathcal{V}_{U, f}^{\bullet}$ of a regular function $f: U \rightarrow \mathbb{C}$ on a smooth $\mathbb{C}$-scheme $U$, with critical locus $X=\operatorname{Crit}(f)$. We prove four main results:

(a) If $\Phi: U \rightarrow U$ is an isomorphism fixing $X$ and compatible with $f$, then the action of $\Phi_{*}$ on $\mathcal{P} \mathcal{V}_{U, f}^{\bullet}$ is multiplication by $\operatorname{det}\left(\left.\mathrm{d} \Phi\right|_{X^{\text {red }}}\right)= \pm 1$.

(b) $\mathcal{P} \mathcal{V}_{U, f}^{\bullet}$ depends up to canonical isomorphism only on $\left(X^{(3)}, f^{(3)}\right)$, for $X^{(3)}$ the third-order thickening of $X$ in $U$, and $f^{(3)}=\left.f\right|_{X^{(3)}}: X^{(3)} \rightarrow \mathbb{C}$.

(c) If $U, V$ are smooth $\mathbb{C}$-schemes, $f: U \rightarrow \mathbb{C}, g: V \rightarrow \mathbb{C}$ are regular, $X=\operatorname{Crit}(f), Y=\operatorname{Crit}(g)$, and $\Phi: U \rightarrow V$ is an embedding with $f=$ $g \circ \Phi$ and $\left.\Phi\right|_{X}: X \rightarrow Y$ an isomorphism, there is a natural isomorphism $\Theta_{\Phi}:\left.\mathcal{P} \mathcal{V}_{U, f}^{\bullet} \rightarrow \Phi\right|_{X} ^{*}\left(\mathcal{P} \mathcal{V}_{V, g}^{\bullet}\right) \otimes_{\mathbb{Z} / 2 \mathbb{Z}} P_{\Phi}$, for $P_{\Phi}$ a principal $\mathbb{Z} / 2 \mathbb{Z}$-bundle on $X$.

(d) If $(X, s)$ is an oriented d-critical locus in the sense of Joyce 23], there is a natural perverse sheaf $P_{X, s}^{\bullet}$ on $X$, such that if $(X, s)$ is locally modelled on $\operatorname{Crit}(f: U \rightarrow \mathbb{C})$ then $P_{X, s}^{\bullet}$ is locally modelled on $\mathcal{P} \mathcal{V}_{U, f}^{\bullet}$.

We also generalize our results to replace $U, X$ by complex analytic spaces, and $\mathcal{P} \mathcal{V}_{U, f}^{\bullet}$ by $\mathscr{D}$-modules or mixed Hodge modules.

We discuss applications of (d) to categorifying Donaldson-Thomas invariants of Calabi-Yau 3-folds, and to defining a 'Fukaya category' of Lagrangians in a complex symplectic manifold using perverse sheaves.
\end{abstract}

\section{Contents}

1 Introduction

2 Background on perverse sheaves 5

2.1 Constructible complexes on $\mathbb{C}$-schemes . . . . . . . . . . . . 6

2.2 Perverse sheaves on $\mathbb{C}$-schemes . . . . . . . . . . . . . . 7

2.3 Nearby cycles and vanishing cycles on $\mathbb{C}$-schemes . . . . . . . . 10

2.4 Perverse sheaves of vanishing cycles on $\mathbb{C}$-schemes . . . . . . . . 12

2.5 Summary of the properties we use in this paper . . . . . . . . 15 
2.6 Perverse sheaves on complex analytic spaces . . . . . . . . . 17

$2.7 \mathscr{D}$-modules on $\mathbb{C}$-schemes and complex analytic spaces . . . . 18

2.8 Mixed Hodge modules: basics . . . . . . . . . . . . . . . . . 18

2.9 Monodromic mixed Hodge modules . . . . . . . . . . . . . . 20

2.10 Mixed Hodge modules of vanishing cycles . . . . . . . . . . 23

3 Action of symmetries on vanishing cycles 26

3.1 Proof of Proposition $3.4 \ldots \ldots \ldots \ldots$. . . . . . . . . . . . . . .

3.2 Theorem 3.1(a): $\operatorname{det}\left(\left.\left.\mathrm{d} \Psi\right|_{X^{\text {red }}} ^{-1} \circ \mathrm{d} \Phi\right|_{X^{\text {red }}}\right)= \pm 1 \ldots \ldots \ldots \ldots$

3.3 Theorem 3.1(b): $\mathcal{P} \mathcal{V}_{\Phi}=\operatorname{det}\left(\left.\left.\mathrm{d} \Psi\right|_{X^{\text {red }}} ^{-1} \circ \mathrm{d} \Phi\right|_{X^{\text {red }}}\right) \cdot \mathcal{P} \mathcal{V}_{\Psi} \ldots \ldots .34$

3.4 D-modules and mixed Hodge modules . . . . . . . . . . 38

4 Dependence of $\mathcal{P} \mathcal{V}_{U, f}^{\bullet}$ on $f \quad 38$

4.1 Proof of Proposition $4.3 \ldots \ldots \ldots \ldots$. . . . . . . . . 41

4.2 Proof of Theorem 4.2 for perverse sheaves on $\mathbb{C}$-schemes . . . . . 45

$4.3 \mathscr{D}$-modules and mixed Hodge modules . . . . . . . . . . . 48

5 Stabilizing perverse sheaves of vanishing cycles 48

5.1 Theorem 5.4(a): the isomorphism $\Theta_{\Phi} \ldots \ldots \ldots \ldots$

5.2 Theorem 5.4(b): $\Theta_{\Phi}$ depends only on $\left.\Phi\right|_{X}: X \rightarrow Y \ldots \ldots \ldots$

5.3 Theorem 5.4 (c): composition of the $\Theta_{\Phi} \ldots \ldots \ldots \ldots \ldots$

$5.4 \mathscr{D}$-modules and mixed Hodge modules . . . . . . . . . . . 59

6 Perverse sheaves on oriented d-critical loci 5

6.1 Background material on d-critical loci . . . . . . . . . . 59

6.2 The main result, and applications . . . . . . . . . . . . 62

6.3 Proof of Theorem 6.9 for perverse sheaves on $\mathbb{C}$-schemes $\ldots . .667$

6.4 D-modules and mixed Hodge modules . . . . . . . . . . 71

A Compatibility results, by Jörg Schürmann 71

$\begin{array}{ll}\text { References } & 74\end{array}$

\section{Introduction}

Let $U$ be a smooth $\mathbb{C}$-scheme and $f: U \rightarrow \mathbb{C}$ a regular function, and write $X=\operatorname{Crit}(f)$, as a $\mathbb{C}$-subscheme of $U$. Then one can define the perverse sheaf of vanishing cycles $\mathcal{P} \mathcal{V}_{U, f}^{\bullet}$ on $X$. Formally, $X=\coprod_{c \in f(X)} X_{c}$, where $X_{c} \subseteq X$ is the open and closed $\mathbb{C}$-subscheme of points $x \in X$ with $f(x)=c$, and $\left.\mathcal{P} \mathcal{V}_{U, f}^{\bullet}\right|_{X_{c}}=$ $\left.\phi_{f-c}^{p}\left(A_{U}[\operatorname{dim} U]\right)\right|_{X_{c}}$ for each $c \in f(X)$, where $A_{U}[\operatorname{dim} U]$ is the constant perverse sheaf on $U$ over a base ring $A$, and $\phi_{f-c}^{p}: \operatorname{Perv}(U) \rightarrow \operatorname{Perv}\left(f^{-1}(c)\right)$ is the vanishing cycle functor for $f-c: U \rightarrow \mathbb{C}$. See $\oint_{2}$ for an introduction to perverse sheaves, and an explanation of this notation.

This paper will prove four main results, Theorems 3.1, 4.2, 5.4 and 6.9, The first three give properties of the $\mathcal{P} \mathcal{V}_{U, f}^{\bullet}$, which we may summarize as follows: 
(a) Let $U, f, X$ be as above, and write $X^{\text {red }}$ for the reduced $\mathbb{C}$-subscheme of $X$. Suppose $\Phi: U \rightarrow U$ is an isomorphism with $f \circ \Phi=f$ and $\left.\Phi\right|_{X}=\operatorname{id}_{X}$. Then $\Phi$ induces a natural isomorphism $\Phi_{*}: \mathcal{P} \mathcal{V}_{U, f}^{\bullet} \rightarrow \mathcal{P} \mathcal{V}_{U, f}^{\bullet}$.

Theorem 3.1 implies that $\left.\mathrm{d} \Phi\right|_{\left.T U\right|_{X^{\text {red }}}}:\left.\left.T U\right|_{X^{\text {red }}} \rightarrow T U\right|_{X^{\text {red }}}$ has determinant $\operatorname{det}\left(\left.\mathrm{d} \Phi\right|_{X^{\text {red }}}\right): X^{\text {red }} \rightarrow \mathbb{C} \backslash\{0\}$ which is a locally constant map $X^{\text {red }} \rightarrow\{ \pm 1\}$, and $\Phi_{*}: \mathcal{P} \mathcal{V}_{U, f}^{\bullet} \rightarrow \mathcal{P} \mathcal{V}_{U, f}^{\bullet}$ is multiplication by $\operatorname{det}\left(\left.\mathrm{d} \Phi\right|_{X^{\text {red }}}\right)$.

In fact Theorem 3.1 proves a more complicated statement, which only requires $\Phi$ to be defined étale locally on $U$.

(b) Let $U, f, X$ be as above, and write $I_{X} \subseteq \mathcal{O}_{U}$ for the sheaf of ideals of regular functions $U \rightarrow \mathbb{C}$ vanishing on $X$. For each $k=1,2, \ldots$, write $X^{(k)}$ for the $k^{\text {th }}$ order thickening of $X$ in $U$, that is, $X^{(k)}$ is the closed $\mathbb{C}$-subscheme of $U$ defined by the vanishing of the sheaf of ideals $I_{X}^{k}$ in $\mathcal{O}_{U}$. Write $f^{(k)}:=\left.f\right|_{X^{(k)}}: X^{(k)} \rightarrow \mathbb{C}$.

Theorem 4.2 says that the perverse sheaf $\mathcal{P} \mathcal{V}_{U, f}^{\bullet}$ depends only on the third-order thickenings $\left(X^{(3)}, f^{(3)}\right)$ up to canonical isomorphism.

As in Remark 4.5, étale locally, $\mathcal{P} \mathcal{V}_{U, f}^{\bullet}$ depends only on $\left(X^{(2)}, f^{(2)}\right)$ up to non-canonical isomorphism, with isomorphisms natural up to sign.

(c) Let $U, V$ be smooth $\mathbb{C}$-schemes, $f: U \rightarrow \mathbb{C}, g: V \rightarrow \mathbb{C}$ be regular, and $X=\operatorname{Crit}(f), Y=\operatorname{Crit}(g)$ as $\mathbb{C}$-subschemes of $U, V$. Let $\Phi: U \hookrightarrow V$ be a closed embedding of $\mathbb{C}$-schemes with $f=g \circ \Phi: U \rightarrow \mathbb{C}$, and suppose $\left.\Phi\right|_{X}: X \rightarrow Y$ is an isomorphism. Then Theorem 5.4 constructs a natural isomorphism of perverse sheaves on $X$ :

$$
\Theta_{\Phi}:\left.\mathcal{P} \mathcal{V}_{U, f}^{\bullet} \longrightarrow \Phi\right|_{X} ^{*}\left(\mathcal{P} \mathcal{V}_{V, g}^{\bullet}\right) \otimes_{\mathbb{Z} / 2 \mathbb{Z}} P_{\Phi}
$$

where $\pi_{\Phi}: P_{\Phi} \rightarrow X$ is a certain principal $\mathbb{Z} / 2 \mathbb{Z}$-bundle on $X$. Writing $N_{U V}$ for the normal bundle of $U$ in $V$, then the Hessian Hess $g$ induces a nondegenerate quadratic form $q_{U V}$ on $\left.N_{U V}\right|_{X}$, and $P_{\Phi}$ parametrizes square roots of $\operatorname{det}\left(q_{U V}\right):\left.\left.K_{U}^{2}\right|_{X} \rightarrow \Phi\right|_{X} ^{*}\left(K_{V}^{2}\right)$.

Theorem 5.4 also shows that the $\Theta_{\Phi}$ in (1.1) are functorial in a suitable sense under compositions of embeddings $\Phi: U \hookrightarrow V, \Psi: V \hookrightarrow W$.

Here (c) is proved by showing that étale locally there exist equivalences $V \simeq$ $U \times \mathbb{C}^{n}$ identifying $\Phi(U)$ with $U \times\{0\}$ and $g: V \rightarrow \mathbb{C}$ with $f \boxplus z_{1}^{2}+\cdots+z_{n}^{2}$ : $U \times \mathbb{C}^{n} \rightarrow \mathbb{C}$, and applying étale local isomorphisms of perverse sheaves

$$
\mathcal{P} \mathcal{V}_{U, f}^{\bullet} \cong \mathcal{P} \mathcal{V}_{U, f}^{\bullet} \stackrel{L}{\otimes} \mathcal{P} \mathcal{V}_{\mathbb{C}^{n}, z_{1}^{2}+\cdots+z_{n}^{2}}^{\bullet} \cong \mathcal{P} \mathcal{V}_{U \times \mathbb{C}^{n}, f \boxplus z_{1}^{2}+\cdots+z_{n}^{2}}^{\bullet} \cong \mathcal{P} \mathcal{V}_{V, g}^{\bullet}
$$

using $\mathcal{P} \mathcal{V}_{\mathbb{C}^{n}, z_{1}^{2}+\cdots+z_{n}^{2}}^{\bullet} \cong A_{\{0\}}$ in the first step, and the Thom-Sebastiani Theorem for perverse sheaves in the second.

Passing from $f: U \rightarrow \mathbb{C}$ to $g=f \boxplus z_{1}^{2}+\cdots+z_{n}^{2}: U \times \mathbb{C}^{n} \rightarrow \mathbb{C}$ is an important idea in singularity theory, as in Arnold et al. 11 for instance. It is known as stabilization, and $f$ and $g$ are called stably equivalent. So, Theorem 5.4 concerns the behaviour of perverse sheaves of vanishing cycles under stabilization. 
Our fourth main result, Theorem [6.9, concerns a new class of geometric objects called $d$-critical loci, introduced in Joyce [23, and explained in 6.1 An (algebraic) d-critical locus $(X, s)$ over $\mathbb{C}$ is a $\mathbb{C}$-scheme $X$ with a section $s$ of a certain natural sheaf $\mathcal{S}_{X}^{0}$ on $X$. A d-critical locus $(X, s)$ may be written Zariski locally as a critical locus $\operatorname{Crit}(f: U \rightarrow \mathbb{C})$ of a regular function $f$ on a smooth $\mathbb{C}$-scheme $U$, and $s$ records some information about $U, f$ (in the notation of (b) above, $s$ remembers $\left.f^{(2)}\right)$. There is also a complex analytic version.

Algebraic d-critical loci are classical truncations of the derived critical loci (more precisely, -1-shifted symplectic derived schemes) introduced in derived algebraic geometry by Pantev, Toën, Vaquié and Vezzosi [42. Theorem 6.9 roughly says that if $(X, s)$ is an algebraic d-critical locus over $\mathbb{C}$ with an 'orientation', then we may define a natural perverse sheaf $P_{X, s}^{\bullet}$ on $X$, such that if $(X, s)$ is locally modelled on $\operatorname{Crit}(f: U \rightarrow \mathbb{C})$ then $P_{X, s}^{\bullet}$ is locally modelled on $\mathcal{P} \mathcal{V}_{U, f}^{\bullet}$. The proof uses Theorem [5.4

These results have exciting applications in the categorification of DonaldsonThomas theory on Calabi-Yau 3-folds, and in defining a new kind of 'Fukaya category' of complex Lagrangians in complex symplectic manifold, which we will discuss at length in Remarks 6.14 and 6.15.

Although we have explained our results only for $\mathbb{C}$-schemes and perverse sheaves upon them, the proofs are quite general and work in several contexts:

(i) Perverse sheaves on $\mathbb{C}$-schemes or complex analytic spaces with coefficients in any well-behaved commutative $\operatorname{ring} A$, such as $\mathbb{Z}, \mathbb{Q}$ or $\mathbb{C}$.

(ii) $\mathscr{D}$-modules on $\mathbb{C}$-schemes or complex analytic spaces.

(iii) Saito's mixed Hodge modules on $\mathbb{C}$-schemes or complex analytic spaces.

We discuss all these in $\sqrt{2}$, before proving our four main results in $\sqrt{3} \sqrt{6}$, Appendix A, by Jörg Schürmann, proves two compatibility results between duality and Thom-Sebastiani type isomorphisms needed in the main text.

This is one of six linked papers [6, 9, 11, 23, with more to come. The best logical order is that the first is Joyce 23] defining d-critical loci, and the second Bussi, Brav and Joyce 9, which proves Darboux-type theorems for the $k$-shifted symplectic derived schemes of Pantev et al. [42, and defines a truncation functor from -1-shifted symplectic derived schemes to algebraic d-critical loci.

This paper is the third in the sequence. Combining our results with [23, 42 gives new results on categorifying Donaldson-Thomas invariants of Calabi-Yau 3 -folds, as in Remark 6.14. In the fourth paper Bussi, Joyce and Meinhardt [11] will generalize the ideas of this paper to motivic Milnor fibres (we explain the relationship between the motivic and cohomological approaches below in Remark 6.10), and deduce new results on motivic Donaldson-Thomas invariants using [2342]. In the fifth, Ben-Bassat, Brav, Bussi and Joyce [6] generalize 9.11] and this paper from (derived) schemes to (derived) Artin stacks.

Sixthly, Bussi [10] will show that if $(S, \omega)$ is a complex symplectic manifold, and $L, M$ are complex Lagrangians in $S$, then the intersection $X=L \cap M$, as a complex analytic subspace of $S$, extends naturally to a complex analytic d-critical locus $(X, s)$. If the canonical bundles $K_{L}, K_{M}$ have square roots 
$K_{L}^{1 / 2}, K_{M}^{1 / 2}$ then $(X, s)$ is oriented, and so Theorem 6.9 below defines a perverse sheaf $P_{L, M}^{\bullet}$ on $X$, which Bussi also constructs directly. As in Remark 6.15, we hope in future work to define a 'Fukaya category' of complex Lagrangians in $(X, \omega)$ in which $\operatorname{Hom}(L, M) \cong \mathbb{H}^{-n}\left(P_{L, M}^{\bullet}\right)$.

Conventions. All $\mathbb{C}$-schemes are assumed separated and of finite type. All complex analytic spaces are Hausdorff and locally of finite type.

Acknowledgements. We would like to thank Oren Ben-Bassat, Alexandru Dimca, Young-Hoon Kiem, Jun Li, Kevin McGerty, Sven Meinhardt, Pierre Schapira, and especially Morihiko Saito and Jörg Schürmann for useful conversations and correspondence, and Jörg Schürmann for a very careful reading of our manuscript, leading to many improvements, as well as providing the Appendix. This research was supported by EPSRC Programme Grant EP/I033343/1.

\section{Background on perverse sheaves}

Perverse sheaves, and the related theories of $\mathscr{D}$-modules and mixed Hodge modules, make sense in several contexts, both algebraic and complex analytic:

(a) Perverse sheaves on $\mathbb{C}$-schemes with coefficients in a ring $A$ (usually $\mathbb{Z}, \mathbb{Q}$ or $\mathbb{C}$ ), as in Beilinson, Bernstein and Deligne [5] and Dimca [14.

(b) Perverse sheaves on complex analytic spaces with coefficients in a ring $A$ (usually $\mathbb{Z}, \mathbb{Q}$ or $\mathbb{C}$ ), as in Dimca [14].

(c) $\mathscr{D}$-modules on $\mathbb{C}$-schemes, as in Borel [8] in the smooth case, and Saito [48] in general.

(d) $\mathscr{D}$-modules on complex manifolds as in Björk [7, and on complex analytic spaces as in Saito [48].

(e) Mixed Hodge modules on $\mathbb{C}$-schemes, as in Saito [45, 47].

(f) Mixed Hodge modules on complex analytic spaces, as in Saito [45,47.

All our main results and proofs work, with minor modifications, in all six settings (a)-(f). As (a) is arguably the simplest and most complete theory, we begin in $2.1-2.4$ with a general introduction to constructible complexes and perverse sheaves on $\mathbb{C}$-schemes, the nearby and vanishing cycle functors, and perverse sheaves of vanishing cycles $P V_{U, f}^{\bullet}$ on $\mathbb{C}$-schemes, following Dimca [14.

Several important properties of perverse sheaves in (a) either do not work, or become more complicated, in settings (b)-(f). Section 2.5 lists the parts of $2.1-4$ that we will use in proofs in this paper, so the reader can check that they do work in (b)-(f). Then $\$ 2.6-10$ give brief discussions of settings (b)-(f), focussing on the differences with (a) in $\$ 2.1-\$ 2.4$.

A good introductory reference on perverse sheaves on $\mathbb{C}$-schemes and complex analytic spaces is Dimca [14. Three other books are Kashiwara and Schapira [27], Schürmann [50, and Hotta, Tanisaki and Takeuchi 21]. Massey [36] and Rietsch [43] are surveys on perverse sheaves, and Beilinson, Bernstein 
and Deligne [5] is an important primary source, who cover both $\mathbb{Q}$-perverse sheaves on $\mathbb{C}$-schemes as in (a), and $\mathbb{Q}_{l}$-perverse sheaves on $\mathbb{K}$-schemes as in (g) below.

Remark 2.1. Two further possible settings, in which not all the results we need are available in the literature, are the following.

(g) Perverse sheaves on $\mathbb{K}$-schemes with coefficients in $\mathbb{Z} / l^{n} \mathbb{Z}, \mathbb{Z}_{l}, \mathbb{Q}_{l}$, or $\overline{\mathbb{Q}}_{l}$ for $l \neq \operatorname{char} \mathbb{K} \neq 2$ a prime, as in Beilinson et al. [5].

(h) $\mathscr{D}$-modules on $\mathbb{K}$-schemes for $\mathbb{K}$ an algebraically closed field, as in Borel [8].

The issue is that the Thom-Sebastiani theorem is not available in these contexts in the generality we need it. Once an appropriate form of this result becomes available, our main theorems will hold also in these two contexts, sometimes under the further assumption that char $\mathbb{K}=0$, needed for the results quoted from [9, 42]. We leave the details to the interested reader.

\subsection{Constructible complexes on $\mathbb{C}$-schemes}

We begin by discussing constructible complexes, following Dimca [14, §2-§4].

Definition 2.2. Fix a well-behaved commutative base ring $A$ (where 'wellbehaved' means that we need assumptions on $A$ such as $A$ is regular noetherian, of finite global dimension or finite Krull dimension, a principal ideal domain, or a Dedekind domain, at various points in the theory), to study sheaves of $A$-modules. For some results $A$ must be a field. Usually we take $A=\mathbb{Z}, \mathbb{Q}$ or $\mathbb{C}$.

Let $X$ be a $\mathbb{C}$-scheme, always assumed of finite type. Write $X^{\text {an }}$ for the set of $\mathbb{C}$-points of $X$ with the complex analytic topology. Consider sheaves of $A$-modules $\mathcal{S}$ on $X^{\text {an }}$. A sheaf $\mathcal{S}$ is called (algebraically) constructible if all the stalks $\mathcal{S}_{x}$ for $x \in X^{\text {an }}$ are finite type $A$-modules, and there is a finite stratification $X^{\text {an }}=\coprod_{j \in J} X_{j}^{\text {an }}$ of $X^{\text {an }}$, where $X_{j} \subseteq X$ for $j \in J$ are $\mathbb{C}$-subschemes of $X$ and $X_{j}^{\text {an }} \subseteq X^{\text {an }}$ the corresponding subsets of $\mathbb{C}$-points, such that $\left.\mathcal{S}\right|_{X_{j}^{\text {an }}}$ is an $A$-local system for all $j \in J$.

Write $D(X)$ for the derived category of complexes $\mathcal{C}^{\bullet}$ of sheaves of $A$-modules on $X^{\text {an }}$. Write $D_{c}^{b}(X)$ for the full subcategory of bounded complexes $\mathcal{C}^{\bullet}$ in $D(X)$ whose cohomology sheaves $\mathcal{H}^{m}\left(\mathcal{C}^{\bullet}\right)$ are constructible for all $m \in \mathbb{Z}$. Then $D(X), D_{c}^{b}(X)$ are triangulated categories. An example of a constructible complex on $X$ is the constant sheaf $A_{X}$ on $X$ with fibre $A$ at each point.

Grothendieck's "six operations on sheaves" $f^{*}, f^{!}, R f_{*}, R f_{!}, \mathcal{R H o m}, \otimes$ act on $D(X)$ preserving the subcategory $D_{c}^{b}(X)$. That is, if $f: X \rightarrow Y$ is a morphism of $\mathbb{C}$-schemes, then we have two different pullback functors $f^{*}, f^{!}: D(Y) \rightarrow D(X)$, which also map $D_{c}^{b}(Y) \rightarrow D_{c}^{b}(X)$. Here $f^{*}$ is called the inverse image [14, §2.3], and $f^{!}$the exceptional inverse image [14, §3.2].

We also have two different pushforward functors $R f_{*}, R f_{!}: D(X) \rightarrow D(Y)$ mapping $D_{c}^{b}(X) \rightarrow D_{c}^{b}(Y)$, where $R f_{*}$ is called the direct image [14, §2.3] and is right adjoint to $f^{*}: D(Y) \rightarrow D(X)$, and $R f_{\text {! }}$ is called the direct image with proper supports [14, §2.3] and is left adjoint to $f^{!}: D(Y) \rightarrow D(X)$. We 
need the assumptions from $\$ 1$ that $X, Y$ are separated and of finite type for $R f_{*}, R f_{!}: D_{c}^{b}(X) \rightarrow D_{c}^{b}(Y)$ to be defined for arbitrary morphisms $f: X \rightarrow Y$.

For $\mathcal{B}^{\bullet}, \mathcal{C}^{\bullet}$ in $D_{c}^{b}(X)$, we may form their derived $\operatorname{Hom} \mathcal{R} \mathcal{H o m}\left(\mathcal{B}^{\bullet}, \mathcal{C}^{\bullet}\right)$ [14, $\S 2.1]$, and left derived tensor product $\mathcal{B}^{\bullet}{ }^{L} \otimes \mathcal{C}^{\bullet}$ in $D_{c}^{b}(X)$, 14, §2.2]. Given $\mathcal{B}^{\bullet} \in$ $D_{c}^{b}(X)$ and $\mathcal{C}^{\bullet} \in D_{c}^{b}(Y)$, we define $\mathcal{B}^{\bullet} \stackrel{L}{\otimes} \mathcal{C}^{\bullet}=\pi_{X}^{*}\left(\mathcal{B}^{\bullet}\right) \stackrel{L}{\otimes} \pi_{Y}^{*}\left(\mathcal{C}^{\bullet}\right)$ in $D_{c}^{b}(X \times Y)$, where $\pi_{X}: X \times Y \rightarrow X, \pi_{Y}: X \times Y \rightarrow Y$ are the projections.

If $X$ is a $\mathbb{C}$-scheme, there is a functor $\mathbb{D}_{X}: D_{c}^{b}(X) \rightarrow D_{c}^{b}(X)^{\text {op }}$ with $\mathbb{D}_{X} \circ$ $\mathbb{D}_{X} \cong \mathrm{id}: D_{c}^{b}(X) \rightarrow D_{c}^{b}(X)$, called Verdier duality. It reverses shifts, that is, $\mathbb{D}_{X}\left(\mathcal{C}^{\bullet}[k]\right)=\left(\mathbb{D}_{X}\left(\mathcal{C}^{\bullet}\right)\right)[-k]$ for $\mathcal{C}^{\bullet}$ in $D_{c}^{b}(X)$ and $k \in \mathbb{Z}$.

Remark 2.3. Note how Definition 2.2 mixes the complex analytic and the complex algebraic: we consider sheaves on $X^{\text {an }}$ in the analytic topology, which are constructible with respect to an algebraic stratification $X=\bigsqcup_{j} X_{j}$.

Here are some properties of all these:

Theorem 2.4. In the following, $X, Y, Z$ are $\mathbb{C}$-schemes, and $f, g$ are morphisms, and all isomorphisms ' $\cong$ ' of functors or objects are canonical.

(i) For $f: X \rightarrow Y$ and $g: Y \rightarrow Z$, there are natural isomorphisms of functors

$$
\begin{aligned}
R(g \circ f)_{*} & \cong R g_{*} \circ R f_{*}, & R(g \circ f)_{!} \cong R g_{!} \circ R f_{!}, \\
(g \circ f)^{*} & \cong f^{*} \circ g^{*}, & (g \circ f)^{!} \cong f^{!} \circ g^{!} .
\end{aligned}
$$

(ii) If $f: X \rightarrow Y$ is proper then $R f_{*} \cong R f_{!}$.

(iii) If $f: X \rightarrow Y$ is étale then $f^{*} \cong f^{!}$. More generally, if $f: X \rightarrow$ $Y$ is smooth of relative (complex) dimension $d$, then $f^{*}[d] \cong f^{!}[-d]$, where $f^{*}[d], f^{!}[-d]$ are the functors $f^{*}, f^{!}$shifted by $\pm d$.

(iv) If $f: X \rightarrow Y$ then $R f_{!} \cong \mathbb{D}_{Y} \circ R f_{*} \circ \mathbb{D}_{X}$ and $f^{!} \cong \mathbb{D}_{X} \circ f^{*} \circ \mathbb{D}_{Y}$.

(v) If $U$ is a smooth $\mathbb{C}$-scheme then $\mathbb{D}_{U}\left(A_{U}\right) \cong A_{U}[2 \operatorname{dim} U]$.

If $X$ is a $\mathbb{C}$-scheme and $\mathcal{C}^{\bullet} \in D_{c}^{b}(X)$, the hypercohomology $\mathbb{H}^{*}\left(\mathcal{C}^{\bullet}\right)$ and compactly-supported hypercohomology $\mathbb{H}_{\mathrm{c}}^{*}\left(\mathcal{C}^{\bullet}\right)$, both graded $A$-modules, are

$$
\mathbb{H}^{k}\left(\mathcal{C}^{\bullet}\right)=H^{k}\left(R \pi_{*}\left(\mathcal{C}^{\bullet}\right)\right) \quad \text { and } \quad \mathbb{H}_{\mathrm{c}}^{k}\left(\mathcal{C}^{\bullet}\right)=H^{k}\left(R \pi_{!}\left(\mathcal{C}^{\bullet}\right)\right) \quad \text { for } k \in \mathbb{Z},
$$

where $\pi: X \rightarrow *$ is projection to a point. If $X$ is proper then $\mathbb{H}^{*}\left(\mathcal{C}^{\bullet}\right) \cong$ $\mathbb{H}_{\mathrm{c}}^{*}\left(\mathcal{C}^{\bullet}\right)$ by Theorem 2.4 (ii). They are related to usual cohomology by $\mathbb{H}^{k}\left(A_{X}\right) \cong$ $H^{k}(X ; A)$ and $\mathbb{H}_{\mathrm{c}}^{k}\left(A_{X}\right) \cong H_{\mathrm{c}}^{k}(X ; A)$. If $A$ is a field then under Verdier duality we have $\mathbb{H}^{k}\left(\mathcal{C}^{\bullet}\right) \cong \mathbb{H}_{\mathrm{c}}^{-k}\left(\mathbb{D}_{X}\left(\mathcal{C}^{\bullet}\right)\right)^{*}$.

\subsection{Perverse sheaves on $\mathbb{C}$-schemes}

Next we review perverse sheaves, following Dimca [14, §5]. 
Definition 2.5. Let $X$ be a $\mathbb{C}$-scheme, and for each $x \in X^{\text {an }}$, let $i_{x}: * \rightarrow X$ map $i_{x}: * \mapsto x$. If $\mathcal{C}^{\bullet} \in D_{c}^{b}(X)$, then the support $\operatorname{supp}^{m} \mathcal{C}^{\bullet}$ and cosupport $\operatorname{cosupp}^{m} \mathcal{C}^{\bullet}$ of $\mathcal{H}^{m}\left(\mathcal{C}^{\bullet}\right)$ for $m \in \mathbb{Z}$ are

$$
\begin{aligned}
\operatorname{supp}^{m} \mathcal{C}^{\bullet} & =\overline{\left\{x \in X^{\text {an }}: \mathcal{H}^{m}\left(i_{x}^{*}\left(\mathcal{C}^{\bullet}\right)\right) \neq 0\right\}}, \\
\operatorname{cosupp}^{m} \mathcal{C}^{\bullet} & =\overline{\left\{x \in X^{\text {an }}: \mathcal{H}^{m}\left(i_{x}^{!}\left(\mathcal{C}^{\bullet}\right)\right) \neq 0\right\}},
\end{aligned}
$$

where $\overline{\{\cdots\}}$ means the closure in $X^{\text {an }}$. If $A$ is a field then $\operatorname{cosupp}^{m} \mathcal{C}^{\bullet}=$ $\operatorname{supp}^{-m} \mathbb{D}_{X}\left(\mathcal{C}^{\bullet}\right)$. We call $\mathcal{C}^{\bullet}$ perverse, or a perverse sheaf, if $\operatorname{dim}_{\mathbb{C}} \operatorname{supp}^{-m} \mathcal{C}^{\bullet} \leqslant m$ and $\operatorname{dim}_{\mathbb{C}} \operatorname{cosupp}{ }^{m} \mathcal{C}^{\bullet} \leqslant m$ for all $m \in \mathbb{Z}$, where by convention $\operatorname{dim}_{\mathbb{C}} \emptyset=-\infty$. Write $\operatorname{Perv}(X)$ for the full subcategory of perverse sheaves in $D_{c}^{b}(X)$. Then $\operatorname{Perv}(X)$ is an abelian category, the heart of a t-structure on $D_{c}^{b}(X)$.

Perverse sheaves have the following properties:

Theorem 2.6. (a) If $A$ is a field then $\operatorname{Perv}(X)$ is noetherian and artinian.

(b) If $A$ is a field then $\mathbb{D}_{X}: D_{c}^{b}(X) \rightarrow D_{c}^{b}(X)$ maps $\operatorname{Perv}(X) \rightarrow \operatorname{Perv}(X)$.

(c) If $i: X \hookrightarrow Y$ is inclusion of a closed $\mathbb{C}$-subscheme, then $R i_{*}, R i_{!}$(which are naturally isomorphic) map $\operatorname{Perv}(X) \rightarrow \operatorname{Perv}(Y)$.

Write $\operatorname{Perv}(Y)_{X}$ for the full subcategory of objects in $\operatorname{Perv}(Y)$ supported on $X$. Then $R i_{*} \cong R i_{\text {! }}$ are equivalences of categories $\operatorname{Perv}(X) \stackrel{\sim}{\longrightarrow} \operatorname{Perv}(Y)_{X}$. The restrictions $\left.i^{*}\right|_{\operatorname{Perv}(Y)_{X}},\left.i^{!}\right|_{\operatorname{Perv}(Y)_{X}}$ map $\operatorname{Perv}(Y)_{X} \rightarrow \operatorname{Perv}(X)$, are naturally isomorphic, and are quasi-inverses for $R i_{*}, R i_{!}: \operatorname{Perv}(X) \rightarrow \operatorname{Perv}(Y)_{X}$.

(d) If $f: X \rightarrow Y$ is étale then $f^{*}$ and $f^{!}$(which are naturally isomorphic) map $\operatorname{Perv}(Y) \rightarrow \operatorname{Perv}(X)$. More generally, if $f: X \rightarrow Y$ is smooth of relative dimension $d$, then $f^{*}[d] \cong f^{!}[-d]$ map $\operatorname{Perv}(Y) \rightarrow \operatorname{Perv}(X)$.

(e) $\stackrel{L}{\otimes}: D_{c}^{b}(X) \times D_{c}^{b}(Y) \rightarrow D_{c}^{b}(X \times Y)$ maps $\operatorname{Perv}(X) \times \operatorname{Perv}(Y) \rightarrow \operatorname{Perv}(X \times Y)$.

(f) Let $U$ be a smooth $\mathbb{C}$-scheme. Then $A_{U}[\operatorname{dim} U]$ is perverse, where $A_{U}$ is the constant sheaf on $U$ with fibre $A$, and $[\operatorname{dim} U]$ means shift by $\operatorname{dim} U$ in the triangulated category $D_{c}^{b}(X)$. Note that Theorem 2.4(v) gives a canonical isomorphism $\mathbb{D}_{U}\left(A_{U}[\operatorname{dim} U]\right) \cong A_{U}[\operatorname{dim} U]$.

When $A=\mathbb{Q}$, so that $\operatorname{Perv}(X)$ is noetherian and artinian by Theorem 2.6(a), the simple objects in $\operatorname{Perv}(X)$ admit a complete description: they are all isomorphic to intersection cohomology complexes $I C_{\bar{V}}(\mathcal{L})$ for $V \subseteq X$ a smooth locally closed $\mathbb{C}$-subscheme and $\mathcal{L} \rightarrow V$ an irreducible $\mathbb{Q}$-local system, [14, §5.4]. Furthermore, if $f: X \rightarrow Y$ is a proper morphism of $\mathbb{C}$-schemes, then the Decomposition Theorem [5, 6.2.5], [14, Th. 5.4.10], [45, Cor. 3] says that, in case $I C_{\bar{V}}(\mathcal{L})$ is of geometric origin, $R f_{*}\left(I C_{\bar{V}}(\mathcal{L})\right)$ is isomorphic to a finite direct sum of shifts of simple objects $I C_{\bar{V}^{\prime}}\left(\mathcal{L}^{\prime}\right)$ in $\operatorname{Perv}(Y)$.

The next theorem is proved by Beilinson et al. [5, Cor. 2.1.23, $\$ 2.2 .19$, \& Th. 3.2.4]. The analogue for $D_{c}^{b}(X)$ or $D(X)$ rather than $\operatorname{Perv}(X)$ is false. One moral is that perverse sheaves behave like sheaves, rather than like complexes.

Theorem 2.7(i) will be used throughout $\$ 3$ - $\$ 6$. Theorem 2.7(ii) will be used only once, in the proof of Theorem 6.9 in 46.3 and we only need Theorem 2.7(ii) to hold in the Zariski topology, rather than the étale topology. 
Theorem 2.7. Let $X$ be $a \mathbb{C}$-scheme. Then perverse sheaves on $X$ form a stack (a kind of sheaf of categories) on $X$ in the étale topology.

Explicitly, this means the following. Let $\left\{u_{i}: U_{i} \rightarrow X\right\}_{i \in I}$ be an étale open cover for $X$, so that $u_{i}: U_{i} \rightarrow X$ is an étale morphism of $\mathbb{C}$-schemes for $i \in I$ with $\coprod_{i} u_{i}$ surjective. Write $U_{i j}=U_{i} \times_{u_{i}, X, u_{j}} U_{j}$ for $i, j \in I$ with projections

$$
\pi_{i j}^{i}: U_{i j} \longrightarrow U_{i}, \quad \pi_{i j}^{j}: U_{i j} \longrightarrow U_{j}, \quad u_{i j}=u_{i} \circ \pi_{i j}^{i}=u_{j} \circ \pi_{i j}^{j}: U_{i j} \longrightarrow X .
$$

Similarly, write $U_{i j k}=U_{i} \times_{X} U_{j} \times_{X} U_{k}$ for $i, j, k \in I$ with projections

$$
\begin{gathered}
\pi_{i j k}^{i j}: U_{i j k} \longrightarrow U_{i j}, \quad \pi_{i j k}^{i k}: U_{i j k} \longrightarrow U_{i k}, \quad \pi_{i j k}^{j k}: U_{i j k} \longrightarrow U_{j k}, \\
\pi_{i j k}^{i}: U_{i j k} \rightarrow U_{i}, \pi_{i j k}^{j}: U_{i j k} \rightarrow U_{j}, \pi_{i j k}^{k}: U_{i j k} \rightarrow U_{k}, u_{i j k}: U_{i j k} \rightarrow X,
\end{gathered}
$$

so that $\pi_{i j k}^{i}=\pi_{i j}^{i} \circ \pi_{i j k}^{i j}, u_{i j k}=u_{i j} \circ \pi_{i j k}^{i j}=u_{i} \circ \pi_{i j k}^{i}$, and so on. All these morphisms $u_{i}, \pi_{i j}^{i}, \ldots, u_{i j k}$ are étale, so by Theorem [2.6(d) $u_{i}^{*} \cong u_{i}^{!}$maps $\operatorname{Perv}(X) \rightarrow \operatorname{Perv}\left(U_{i}\right)$, and similarly for $\pi_{i j}^{i}, \ldots, u_{i j k}$. With this notation:

(i) Suppose $\mathcal{P}^{\bullet}, \mathcal{Q}^{\bullet} \in \operatorname{Perv}(X)$, and we are given $\alpha_{i}: u_{i}^{*}\left(\mathcal{P}^{\bullet}\right) \rightarrow u_{i}^{*}\left(\mathcal{Q}^{\bullet}\right)$ in $\operatorname{Perv}\left(U_{i}\right)$ for all $i \in I$ such that for all $i, j \in I$ we have

$$
\left(\pi_{i j}^{i}\right)^{*}\left(\alpha_{i}\right)=\left(\pi_{i j}^{i}\right)^{*}\left(\alpha_{j}\right): u_{i j}^{*}\left(\mathcal{P}^{\bullet}\right) \longrightarrow u_{i j}^{*}\left(\mathcal{Q}^{\bullet}\right) .
$$

Then there is a unique $\alpha: \mathcal{P}^{\bullet} \rightarrow \mathcal{Q}^{\bullet}$ in $\operatorname{Perv}(X)$ with $\alpha_{i}=u_{i}^{*}(\alpha)$ for all $i \in I$.

(ii) Suppose we are given objects $\mathcal{P}_{i}^{\bullet} \in \operatorname{Perv}\left(U_{i}\right)$ for all $i \in I$ and isomorphisms $\alpha_{i j}:\left(\pi_{i j}^{i}\right)^{*}\left(\mathcal{P}_{i}^{\bullet}\right) \rightarrow\left(\pi_{i j}^{j}\right)^{*}\left(\mathcal{P}_{j}^{\bullet}\right)$ in $\operatorname{Perv}\left(U_{i j}\right)$ for all $i, j \in I$ with $\alpha_{i i}=$ id and

$$
\left(\pi_{i j k}^{j k}\right)^{*}\left(\alpha_{j k}\right) \circ\left(\pi_{i j k}^{i j}\right)^{*}\left(\alpha_{i j}\right)=\left(\pi_{i j k}^{i k}\right)^{*}\left(\alpha_{i k}\right):\left(\pi_{i j k}^{i}\right)^{*}\left(\mathcal{P}_{i}\right) \longrightarrow\left(\pi_{i j k}^{k}\right)^{*}\left(\mathcal{P}_{k}\right)
$$

in $\operatorname{Perv}\left(U_{i j k}\right)$ for all $i, j, k \in I$. Then there exists $\mathcal{P}^{\bullet}$ in $\operatorname{Perv}(X)$, unique up to canonical isomorphism, with isomorphisms $\beta_{i}: u_{i}^{*}\left(\mathcal{P}^{\bullet}\right) \rightarrow \mathcal{P}_{i}^{\bullet}$ for each $i \in I$, satisfying $\alpha_{i j} \circ\left(\pi_{i j}^{i}\right)^{*}\left(\beta_{i}\right)=\left(\pi_{i j}^{j}\right)^{*}\left(\beta_{j}\right): u_{i j}^{*}\left(\mathcal{P}^{\bullet}\right) \rightarrow\left(\pi_{i j}^{j}\right)^{*}\left(\mathcal{P}_{j}^{\bullet}\right)$ for all $i, j \in I$.

We will need the following proposition in $\$ 3.3$ to prove Theorem 3.1(b). Most of it is setting up notation, only the last part $\left.\alpha\right|_{X^{\prime}}=\left.\beta\right|_{X^{\prime}}$ is nontrivial.

Proposition 2.8. Let $W, X$ be $\mathbb{C}$-schemes, $x \in X$, and $\pi_{\mathbb{C}}: W \rightarrow \mathbb{C}, \pi_{X}$ : $W \rightarrow X, \iota: \mathbb{C} \rightarrow W$ morphisms, such that $\pi_{\mathbb{C}} \times \pi_{X}: W \rightarrow \mathbb{C} \times X$ is étale, and $\pi_{\mathbb{C}} \circ \iota=\mathrm{id}_{\mathbb{C}}: \mathbb{C} \rightarrow \mathbb{C}$, and $\pi_{X} \circ \iota(t)=x$ for all $t \in \mathbb{C}$. Write $W_{t}=\pi_{\mathbb{C}}^{-1}(t) \subset W$ for each $t \in \mathbb{C}$, and $j_{t}: W_{t} \hookrightarrow W$ for the inclusion. Then $\left.\pi_{X}\right|_{W_{t}}=\pi_{X} \circ j_{t}$ : $W_{t} \rightarrow X$ is étale, and $\iota(t) \in W_{t}$ with $\left.\pi_{X}\right|_{W_{t}}(\iota(t))=x$, so we may think of $W_{t}$ for $t \in \mathbb{C}$ as a 1-parameter family of étale open neighbourhoods of $x$ in $X$.

Let $\mathcal{P}^{\bullet}, \mathcal{Q}^{\bullet} \in \operatorname{Perv}(X)$, so that by Theorem 2.6(d) as $\pi_{X}$ is smooth of relative dimension 1 and $\left.\pi_{X}\right|_{W_{t}}$ is étale, we have $\pi_{X}^{*}[1]\left(\mathcal{P}^{\bullet}\right) \in \operatorname{Perv}(W)$ and $\left.\pi_{X}\right|_{W_{t}} ^{*}\left(\mathcal{P}^{\bullet}\right)=j_{t}^{*}[-1]\left(\pi_{X}^{*}[1]\left(\mathcal{P}^{\bullet}\right)\right) \in \operatorname{Perv}\left(W_{t}\right)$, and similarly for $\mathcal{Q}^{\bullet}$.

Suppose $\alpha, \beta: \mathcal{P}^{\bullet} \rightarrow \mathcal{Q}^{\bullet}$ in $\operatorname{Perv}(X)$ and $\gamma: \pi_{X}^{*}[1]\left(\mathcal{P}^{\bullet}\right) \rightarrow \pi_{X}^{*}[1]\left(\mathcal{Q}^{\bullet}\right)$ in $\operatorname{Perv}(W)$ are morphisms such that $\left.\pi_{X}\right|_{W_{0}} ^{*}(\alpha)=j_{0}^{*}[-1](\gamma)$ in $\operatorname{Perv}\left(W_{0}\right)$ and $\left.\pi_{X}\right|_{W_{1}} ^{*}(\beta)=j_{1}^{*}[-1](\gamma)$ in $\operatorname{Perv}\left(W_{1}\right)$. Then there exists a Zariski open neighbourhood $X^{\prime}$ of $x$ in $X$ such that $\left.\alpha\right|_{X^{\prime}}=\left.\beta\right|_{X^{\prime}}:\left.\left.\mathcal{P}^{\bullet}\right|_{X^{\prime}} \rightarrow \mathcal{Q}^{\bullet}\right|_{X^{\prime}}$. 
Here we should think of $j_{t}^{*}[-1](\gamma)$ for $t \in \mathbb{C}$ as a family of perverse sheaf morphisms $\mathcal{P}^{\bullet} \rightarrow \mathcal{Q}^{\bullet}$, defined near $x$ in $X$ locally in the étale topology. But morphisms of perverse sheaves are discrete (to see this, note that we can take $A=\mathbb{Z})$, so as $j_{t}^{*}[-1](\gamma)$ depends continuously on $t$, it should be locally constant in $t$ near $x$, in a suitable sense. The conclusion $\left.\alpha\right|_{X^{\prime}}=\left.\beta\right|_{X^{\prime}}$ essentially says that $j_{0}^{*}[-1](\gamma)=j_{1}^{*}[-1](\gamma)$ near $x$.

If $P \rightarrow X$ is a principal $\mathbb{Z} / 2 \mathbb{Z}$-bundle on a $\mathbb{C}$-scheme $X$, and $\mathcal{Q}^{\bullet} \in \operatorname{Perv}(X)$, we will define a perverse sheaf $\mathcal{Q}^{\bullet} \otimes_{\mathbb{Z} / 2 \mathbb{Z}} P$, which will be important in 5 .

Definition 2.9. Let $X$ be a $\mathbb{C}$-scheme. A principal $\mathbb{Z} / 2 \mathbb{Z}$-bundle $P \rightarrow X$ is a proper, surjective, étale morphism of $\mathbb{C}$-schemes $\pi: P \rightarrow X$ together with a free involution $\sigma: P \rightarrow P$, such that the orbits of $\mathbb{Z} / 2 \mathbb{Z}=\{1, \sigma\}$ are the fibres of $\pi$. We will use the ideas of isomorphism of principal bundles $\iota: P \rightarrow P^{\prime}$, section $s: X \rightarrow P$, tensor product $P \otimes_{\mathbb{Z} / 2 \mathbb{Z}} P^{\prime}$, and pullback $f^{*}(P) \rightarrow W$ under a $\mathbb{C}$-scheme morphism $f: W \rightarrow X$, all of which are defined in the obvious ways.

Let $P \rightarrow X$ be a principal $\mathbb{Z} / 2 \mathbb{Z}$-bundle. Write $\mathcal{L}_{P} \in D_{c}^{b}(X)$ for the rank one $A$-local system on $X$ induced from $P$ by the nontrivial representation of $\mathbb{Z} / 2 \mathbb{Z} \cong\{ \pm 1\}$ on $A$. It is characterized by $\pi_{*}\left(A_{P}\right) \cong A_{X} \oplus \mathcal{L}_{P}$. For each $\mathcal{Q}^{\bullet} \in D_{c}^{b}(X)$, write $\mathcal{Q}^{\bullet} \otimes_{\mathbb{Z} / 2 \mathbb{Z}} P \in D_{c}^{b}(X)$ for $\mathcal{Q}^{\bullet}{ }^{L} \otimes \mathcal{L}_{P}$, and call it $\mathcal{Q}^{\bullet}$ twisted by $P$. If $\mathcal{Q}^{\bullet}$ is perverse then $\mathcal{Q}^{\bullet} \otimes_{\mathbb{Z} / 2 \mathbb{Z}} P$ is perverse.

Perverse sheaves and complexes twisted by principal $\mathbb{Z} / 2 \mathbb{Z}$-bundles have the obvious functorial behaviour. For example, if $P \rightarrow X, P^{\prime} \rightarrow X$ are principal $\mathbb{Z} / 2 \mathbb{Z}$-bundles and $\mathcal{Q}^{\bullet} \in D_{c}^{b}(X)$ there is a canonical isomorphism $\left(\mathcal{Q}^{\bullet} \otimes_{\mathbb{Z} / 2 \mathbb{Z}}\right.$ $P) \otimes_{\mathbb{Z} / 2 \mathbb{Z}} P^{\prime} \cong \mathcal{Q}^{\bullet} \otimes_{\mathbb{Z} / 2 \mathbb{Z}}\left(P \otimes_{\mathbb{Z} / 2 \mathbb{Z}} P^{\prime}\right)$, and if $f: W \rightarrow X$ is a $\mathbb{C}$-scheme morphism there is a canonical isomorphism $f^{*}\left(\mathcal{Q}^{\bullet} \otimes_{\mathbb{Z} / 2 \mathbb{Z}} P\right) \cong f^{*}\left(\mathcal{Q}^{\bullet}\right) \otimes_{\mathbb{Z} / 2 \mathbb{Z}} f^{*}(P)$.

\subsection{Nearby cycles and vanishing cycles on $\mathbb{C}$-schemes}

We explain nearby cycles and vanishing cycles, as in Dimca [14, §4.2]. The definition is complex analytic, $\widetilde{X_{*}^{\text {an }}}, \widetilde{\mathbb{C}^{*}}$ in $(2.2)$ do not come from $\mathbb{C}$-schemes.

Definition 2.10. Let $X$ be a $\mathbb{C}$-scheme, and $f: X \rightarrow \mathbb{C}$ a regular function. Define $X_{0}=f^{-1}(0)$, as a $\mathbb{C}$-subscheme of $X$, and $X_{*}=X \backslash X_{0}$. Consider the commutative diagram of complex analytic spaces:

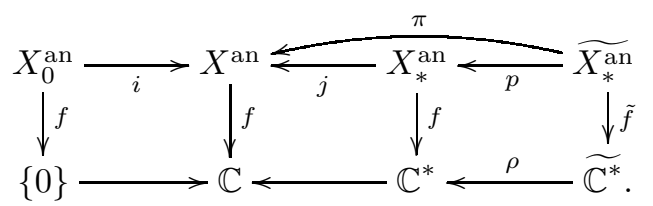

Here $X^{\text {an }}, X_{0}^{\text {an }}, X_{*}^{\text {an }}$ are the complex analytic spaces associated to the $\mathbb{C}$-schemes $X_{0}, X, X_{*}$, and $i: X_{0}^{\text {an }} \hookrightarrow X^{\text {an }}, j: X_{*}^{\text {an }} \hookrightarrow X^{\text {an }}$ are the inclusions, $\rho: \widetilde{\mathbb{C}^{*}} \rightarrow \mathbb{C}^{*}$ is the universal cover of $\mathbb{C}^{*}=\mathbb{C} \backslash\{0\}$, and $\widetilde{X_{*}^{\text {an }}}=X_{*}^{\text {an }} \times_{f, \mathbb{C}^{*}, \rho} \widetilde{\mathbb{C}^{*}}$ the corresponding cover of $X_{*}^{\text {an }}$, with covering map $p: \widetilde{X_{*}^{\text {an }}} \rightarrow X_{*}^{\text {an }}$, and $\pi=j \circ p$.

As in \$2.6. the triangulated categories $D(X), D_{c}^{b}(X)$ and six operations $f^{*}, f^{!}, R f_{*}, R f_{!}, \mathcal{R H}$ Hom,$\stackrel{L}{\otimes}$ also make sense for complex analytic spaces. So 
we can define the nearby cycle functor $\psi_{f}: D_{c}^{b}(X) \rightarrow D_{c}^{b}\left(X_{0}\right)$ to be $\psi_{f}=$ $i^{*} \circ R \pi_{*} \circ \pi^{*}$. Since this definition goes via $\widehat{X_{*}^{\text {an }}}$ which is not a $\mathbb{C}$-scheme, it is not obvious that $\psi_{f}$ maps to (algebraically) constructible complexes $D_{c}^{b}\left(X_{0}\right)$ rather than just to $D\left(X_{0}\right)$, but it does [14, p. 103], [27, p. 352].

There is a natural transformation $\Xi: i^{*} \Rightarrow \psi_{f}$ between the functors $i^{*}, \psi_{f}$ : $D_{c}^{b}(X) \rightarrow D_{c}^{b}\left(X_{0}\right)$. The vanishing cycle functor $\phi_{f}: D_{c}^{b}(X) \rightarrow D_{c}^{b}\left(X_{0}\right)$ is a functor such that for every $\mathcal{C}^{\bullet}$ in $D_{c}^{b}(X)$ we have a distinguished triangle

$$
i^{*}\left(\mathcal{C}^{\bullet}\right) \stackrel{\Xi\left(\mathcal{C}^{\bullet}\right)}{\longrightarrow} \psi_{f}\left(\mathcal{C}^{\bullet}\right) \longrightarrow \phi_{f}\left(\mathcal{C}^{\bullet}\right) \stackrel{[+1]}{\longrightarrow} i^{*}\left(\mathcal{C}^{\bullet}\right)
$$

in $D_{c}^{b}\left(X_{0}\right)$. Following Dimca [14, p. 108], we write $\psi_{f}^{p}, \phi_{f}^{p}$ for the shifted functors $\psi_{f}[-1], \phi_{f}[-1]: D_{c}^{b}(X) \rightarrow D_{c}^{b}\left(X_{0}\right)$.

The generator of $\mathbb{Z}=\pi_{1}\left(\mathbb{C}^{*}\right)$ on $\widetilde{\mathbb{C}^{*}}$ induces a deck transformation $\delta_{\mathbb{C}^{*}}$ : $\widetilde{\mathbb{C}^{*}} \rightarrow \widetilde{\mathbb{C}^{*}}$ which lifts to a deck transformation $\delta_{X^{*}}: \widetilde{X^{*}} \rightarrow \widetilde{X^{*}}$ with $p \circ \delta_{X^{*}}=p$ and $\tilde{f} \circ \delta_{X^{*}}=\delta_{\mathbb{C}^{*}} \circ \tilde{f}$. As in [14, p. 103, p. 105], we can use $\delta_{X^{*}}$ to define natural transformations $M_{X, f}: \psi_{f}^{p} \Rightarrow \psi_{f}^{p}$ and $M_{X, f}: \phi_{f}^{p} \Rightarrow \phi_{f}^{p}$, called monodromy.

Alternative definitions of $\psi_{f}, \phi_{f}$ in terms of specialization and microlocalization functors are given by Kashiwara and Schapira [27, Prop. 8.6.3]. Here are some properties of nearby and vanishing cycles. Parts (i),(ii) can be found in Dimca [14, Th. 5.2.21 \& Prop. 4.2.11]. Part (iv) is proved by Massey 37; compare also Proposition A.1 in the Appendix.

Theorem 2.11. (i) If $X$ is a $\mathbb{C}$-scheme and $f: X \rightarrow \mathbb{C}$ is regular, then $\psi_{f}^{p}, \phi_{f}^{p}: D_{c}^{b}(X) \rightarrow D_{c}^{b}\left(X_{0}\right)$ both map $\operatorname{Perv}(X) \rightarrow \operatorname{Perv}\left(X_{0}\right)$.

(ii) Let $\Phi: X \rightarrow Y$ be a proper morphism of $\mathbb{C}$-schemes, and $g: Y \rightarrow \mathbb{C}$ be regular. Write $f=g \circ \Phi: X \rightarrow \mathbb{C}, X_{0}=f^{-1}(0) \subseteq X, Y_{0}=g^{-1}(0) \subseteq Y$, and $\Phi_{0}=\left.\Phi\right|_{X_{0}}: X_{0} \rightarrow Y_{0}$. Then we have natural isomorphisms

$$
R\left(\Phi_{0}\right)_{*} \circ \psi_{f}^{p} \cong \psi_{g}^{p} \circ R \Phi_{*} \quad \text { and } \quad R\left(\Phi_{0}\right)_{*} \circ \phi_{f}^{p} \cong \phi_{g}^{p} \circ R \Phi_{*} .
$$

Note too that $R \Phi_{*} \cong R \Phi_{\text {! }}$ and $R\left(\Phi_{0}\right)_{*} \cong R\left(\Phi_{0}\right)_{\text {! }}$, as $\Phi, \Phi_{0}$ are proper.

(iii) Let $\Phi: X \rightarrow Y$ be an étale morphism of $\mathbb{C}$-schemes, and $g: Y \rightarrow \mathbb{C}$ be regular. Write $f=g \circ \Phi: X \rightarrow \mathbb{C}, X_{0}=f^{-1}(0) \subseteq X, Y_{0}=g^{-1}(0) \subseteq Y$, and $\Phi_{0}=\left.\Phi\right|_{X_{0}}: X_{0} \rightarrow Y_{0}$. Then we have natural isomorphisms

$$
\Phi_{0}^{*} \circ \psi_{f}^{p} \cong \psi_{g}^{p} \circ \Phi^{*} \quad \text { and } \quad \Phi_{0}^{*} \circ \phi_{f}^{p} \cong \phi_{g}^{p} \circ \Phi^{*} .
$$

Note too that $\Phi^{*} \cong \Phi^{!}$and $\Phi_{0}^{*} \cong \Phi_{0}^{!}$, as $\Phi, \Phi_{0}$ are étale.

More generally, if $\Phi: X \rightarrow Y$ is smooth of relative (complex) dimension d and $g, f, X_{0}, Y_{0}, \Phi_{0}$ are as above then we have natural isomorphisms

$$
\Phi_{0}^{*}[d] \circ \psi_{f}^{p} \cong \psi_{g}^{p} \circ \Phi^{*}[d] \quad \text { and } \quad \Phi_{0}^{*}[d] \circ \phi_{f}^{p} \cong \phi_{g}^{p} \circ \Phi^{*}[d] .
$$

Note too that $\Phi^{*}[d] \cong \Phi^{!}[-d]$ and $\Phi_{0}^{*}[d] \cong \Phi_{0}^{!}[-d]$.

(iv) If $X$ is a $\mathbb{C}$-scheme and $f: X \rightarrow \mathbb{C}$ is regular, then there are natural isomorphisms $\psi_{f}^{p} \circ \mathbb{D}_{X} \cong \mathbb{D}_{X_{0}} \circ \psi_{f}^{p}$ and $\phi_{f}^{p} \circ \mathbb{D}_{X} \cong \mathbb{D}_{X_{0}} \circ \phi_{f}^{p}$. 


\subsection{Perverse sheaves of vanishing cycles on $\mathbb{C}$-schemes}

We can now define the main subject of this paper, the perverse sheaf of vanishing cycles $\mathcal{P} \mathcal{V}_{U, f}^{\bullet}$ for a regular function $f: U \rightarrow \mathbb{C}$.

Definition 2.12. Let $U$ be a smooth $\mathbb{C}$-scheme, and $f: U \rightarrow \mathbb{C}$ a regular function. Write $X=\operatorname{Crit}(f)$, as a closed $\mathbb{C}$-subscheme of $U$. Then as a map of topological spaces, $\left.f\right|_{X}: X \rightarrow \mathbb{C}$ is locally constant, with finite image $f(X)$, so we have a decomposition $X=\coprod_{c \in f(X)} X_{c}$, for $X_{c} \subseteq X$ the open and closed $\mathbb{C}$-subscheme with $f(x)=c$ for each $\mathbb{C}$-point $x \in X_{c}$.

(Note that if $X$ is non-reduced, then $\left.f\right|_{X}: X \rightarrow \mathbb{C}$ need not be locally constant as a morphism of $\mathbb{C}$-schemes, but $\left.f\right|_{X^{\text {red }}}: X^{\text {red }} \rightarrow \mathbb{C}$ is locally constant, where $X^{\text {red }}$ is the reduced $\mathbb{C}$-subscheme of $X$. Since $X, X^{\text {red }}$ have the same topological space, $\left.f\right|_{X}: X \rightarrow \mathbb{C}$ is locally constant on topological spaces.)

For each $c \in \mathbb{C}$, write $U_{c}=f^{-1}(c) \subseteq U$. Then as in $\$ 2.3$, we have a vanishing cycle functor $\phi_{f-c}^{p}: \operatorname{Perv}(U) \rightarrow \operatorname{Perv}\left(U_{c}\right)$. So we may form $\phi_{f-c}^{p}\left(A_{U}[\operatorname{dim} U]\right)$ in $\operatorname{Perv}\left(U_{c}\right)$, since $A_{U}[\operatorname{dim} U] \in \operatorname{Perv}(U)$ by Theorem 2.6(f). One can show $\phi_{f-c}^{p}\left(A_{U}[\operatorname{dim} U]\right)$ is supported on the closed subset $X_{c}=\operatorname{Crit}(f) \cap U_{c}$ in $U_{c}$, where $X_{c}=\emptyset$ unless $c \in f(X)$. That is, $\phi_{f-c}^{p}\left(A_{U}[\operatorname{dim} U]\right)$ lies in $\operatorname{Perv}\left(U_{c}\right)_{X_{c}}$.

But Theorem 2.6 (c) says $\operatorname{Perv}\left(U_{c}\right)_{X_{c}}$ and $\operatorname{Perv}\left(X_{c}\right)$ are equivalent categories, so we may regard $\phi_{f-c}^{p}\left(A_{U}[\operatorname{dim} U]\right)$ as a perverse sheaf on $X_{c}$. That is, we can consider $\left.\phi_{f-c}^{p}\left(A_{U}[\operatorname{dim} U]\right)\right|_{X_{c}}=i_{X_{c}, U_{c}}^{*}\left(\phi_{f-c}^{p}\left(A_{U}[\operatorname{dim} U]\right)\right)$ in $\operatorname{Perv}\left(X_{c}\right)$, where $i_{X_{c}, U_{c}}: X_{c} \rightarrow U_{c}$ is the inclusion morphism.

As $X=\coprod_{c \in f(X)} X_{c}$ with each $X_{c}$ open and closed in $X$, we have $\operatorname{Perv}(X)=$ $\bigoplus_{c \in f(X)} \operatorname{Perv}\left(X_{c}\right)$. Define the perverse sheaf of vanishing cycles $\mathcal{P} \mathcal{V}_{U, f}^{\bullet}$ of $U, f$ in $\operatorname{Perv}(X)$ to be $\mathcal{P} \mathcal{V}_{U, f}^{\bullet}=\left.\bigoplus_{c \in f(X)} \phi_{f-c}^{p}\left(A_{U}[\operatorname{dim} U]\right)\right|_{X_{c}}$. That is, $\mathcal{P} \mathcal{V}_{U, f}^{\bullet}$ is the unique perverse sheaf on $X=\operatorname{Crit}(f)$ with $\left.\mathcal{P} \mathcal{V}_{U, f}^{\bullet}\right|_{X_{c}}=\left.\phi_{f-c}^{p}\left(A_{U}[\operatorname{dim} U]\right)\right|_{X_{c}}$ for all $c \in f(X)$.

Under Verdier duality, we have $A_{U}[\operatorname{dim} U] \cong \mathbb{D}_{U}\left(A_{U}[\operatorname{dim} U]\right)$ by Theorem 2.6(f), so $\phi_{f-c}^{p}\left(A_{U}[\operatorname{dim} U]\right) \cong \mathbb{D}_{U_{c}}\left(\phi_{f-c}^{p}\left(A_{U}[\operatorname{dim} U]\right)\right)$ by Theorem2.11(iv). Applying $i_{X_{c}, U_{c}}^{*}$ and using $\mathbb{D}_{X_{c}} \circ i_{X_{c}, U_{c}}^{*} \cong i_{X_{c}, U_{c}}^{!} \circ \mathbb{D}_{U_{c}}$ by Theorem 2.4(iv) and $i_{X_{c}, U_{c}}^{!} \cong i_{X_{c}, U_{c}}^{*}$ on $\operatorname{Perv}\left(U_{c}\right)_{X_{c}}$ by Theorem 2.6 (c) also gives

$$
\left.\phi_{f-c}^{p}\left(A_{U}[\operatorname{dim} U]\right)\right|_{X_{c}} \cong \mathbb{D}_{X_{c}}\left(\left.\phi_{f-c}^{p}\left(A_{U}[\operatorname{dim} U]\right)\right|_{X_{c}}\right)
$$

Summing over all $c \in f(X)$ yields a canonical isomorphism

$$
\sigma_{U, f}: \mathcal{P} \mathcal{V}_{U, f}^{\bullet} \stackrel{\cong}{\longrightarrow} \mathbb{D}_{X}\left(\mathcal{P} \mathcal{V}_{U, f}^{\bullet}\right)
$$

For $c \in f(X)$, we have a monodromy operator $M_{U, f-c}: \phi_{f-c}^{p}\left(A_{U}[\operatorname{dim} U]\right)$ $\rightarrow \phi_{f-c}^{p}\left(A_{U}[\operatorname{dim} U]\right)$, which restricts to $\left.\phi_{f-c}^{p}\left(A_{U}[\operatorname{dim} U]\right)\right|_{X_{c}}$. Define the twisted monodromy operator $\tau_{U, f}: \mathcal{P} \mathcal{V}_{U, f}^{\bullet} \rightarrow \mathcal{P} \mathcal{V}_{U, f}^{\bullet}$ by

$$
\begin{aligned}
\left.\tau_{U, f}\right|_{X_{c}}=\left.(-1)^{\operatorname{dim} U} M_{U, f-c}\right|_{X_{c}}: \\
\left.\left.\phi_{f-c}^{p}\left(A_{U}[\operatorname{dim} U]\right)\right|_{X_{c}} \longrightarrow \phi_{f-c}^{p}\left(A_{U}[\operatorname{dim} U]\right)\right|_{X_{c}}
\end{aligned}
$$


for each $c \in f(X)$. Here 'twisted' refers to the sign $(-1)^{\operatorname{dim} U}$ in (2.7). We include this sign change as it makes monodromy act naturally under transformations which change dimension - without it, equation (5.15) below would only commute up to a sign $(-1)^{\operatorname{dim} V-\operatorname{dim} U}$, not commute - and it normalizes the monodromy of any nondegenerate quadratic form to be the identity, as in (2.13). The sign $(-1)^{\operatorname{dim} U}$ also corresponds to the twist ' $\left(\frac{1}{2} \operatorname{dim} U\right)$ ' in the definition (2.24) of the mixed Hodge module of vanishing cycles $\mathcal{H} \mathcal{V}_{U, f}^{\bullet}$ in $\$ 2.10$

The (compactly-supported) hypercohomology $\mathbb{H}^{*}\left(\mathcal{P} \mathcal{V}_{U, f}^{\bullet}\right), \mathbb{H}_{\mathrm{c}}^{*}\left(\mathcal{P} \mathcal{V}_{U, f}^{\bullet}\right)$ from (2.1) is an important invariant of $U, f$. If $A$ is a field then the isomorphism $\sigma_{U, f}$ in (2.6) implies that $\mathbb{H}^{k}\left(\mathcal{P} \mathcal{V}_{U, f}^{\bullet}\right) \cong \mathbb{H}_{\mathrm{c}}^{-k}\left(\mathcal{P} \mathcal{V}_{U, f}^{\bullet}\right)^{*}$, a form of Poincaré duality.

We defined $\mathcal{P} \mathcal{V}_{U, f}^{\bullet}$ in perverse sheaves over a base ring $A$. Writing $\mathcal{P} \mathcal{V}_{U, f}^{\bullet}(A)$ to denote the base ring, one can show that $\mathcal{P} \mathcal{V}_{U, f}^{\bullet}(A) \cong \mathcal{P} \mathcal{V}_{U, f}^{\bullet}(\mathbb{Z})^{L} \otimes_{\mathbb{Z}} A$. Thus, we may as well take $A=\mathbb{Z}$, or $A=\mathbb{Q}$ if we want $A$ to be a field, since the case of general $A$ contains no more information.

There is a Thom-Sebastiani Theorem for perverse sheaves, due to Massey [35] and Schürmann [50, Cor. 1.3.4]. Applied to $\mathcal{P} \mathcal{V}_{U, f}^{\bullet}$, it yields:

Theorem 2.13. Let $U, V$ be smooth $\mathbb{C}$-schemes and $f: U \rightarrow \mathbb{C}, g: V \rightarrow \mathbb{C}$ be regular, so that $f \boxplus g: U \times V \rightarrow \mathbb{C}$ is regular with $(f \boxplus g)(u, v):=f(u)+g(v)$. Set $X=\operatorname{Crit}(f)$ and $Y=\operatorname{Crit}(g)$ as $\mathbb{C}$-subschemes of $U, V$, so that $\operatorname{Crit}(f \boxplus g)=$ $X \times Y$. Then there is a natural isomorphism

$$
\mathcal{T} \mathcal{S}_{U, f, V, g}: \mathcal{P} \mathcal{V}_{U \times V, f \boxplus g}^{\bullet} \longrightarrow \mathcal{P} \mathcal{V}_{U, f}^{\bullet} \stackrel{L}{ } \mathcal{P} \mathcal{V}_{V, g}^{\bullet}
$$

in $\operatorname{Perv}(X \times Y)$, such that the following diagrams commute:

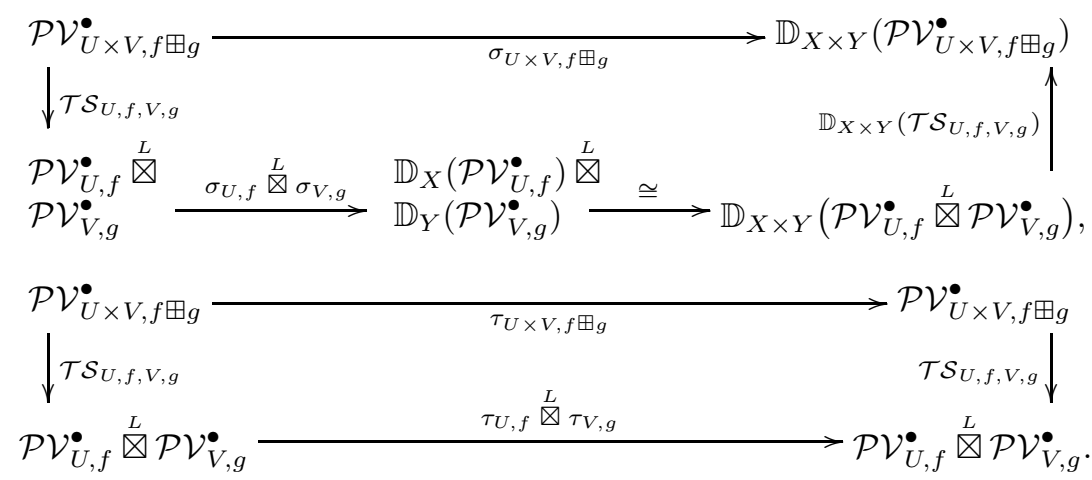

The next example will be important later.

Example 2.14. Define $f: \mathbb{C}^{n} \rightarrow \mathbb{C}$ by $f\left(z_{1}, \ldots, z_{n}\right)=z_{1}^{2}+\cdots+z_{n}^{2}$ for $n>1$. Then $\operatorname{Crit}(f)=\{0\}$, so $\mathcal{P} \mathcal{V}_{\mathbb{C}^{n}, z_{1}^{2}+\cdots+z_{n}^{2}}^{\bullet}=\left.\phi_{f}^{p}\left(A_{\mathbb{C}^{n}}[n]\right)\right|_{\{0\}}$ is a perverse sheaf on the point $\{0\}$. Following Dimca [14, Prop. 4.2.2, Ex. 4.2.3 \& Ex. 4.2.6], we find that there is a canonical isomorphism

$$
\mathcal{P} \mathcal{V}_{\mathbb{C}^{n}, z_{1}^{2}+\cdots+z_{n}^{2}}^{\bullet} H^{n-1}\left(M F_{f}(0) ; A\right) \otimes_{A} A_{\{0\}},
$$


where $M F_{f}(0)$ is the Milnor fibre of $f$ at 0 , as in [14, p. 103]. Since $f(z)=$ $z_{1}^{2}+\cdots+z_{n}^{2}$ is homogeneous, we see that

$$
M F_{f}(0) \cong\left\{\left(z_{1}, \ldots, z_{n}\right) \in \mathbb{C}^{n}: f\left(z_{1}, \ldots, z_{n}\right)=1\right\} \cong T^{*} \mathcal{S}^{n-1},
$$

so that $H^{n-1}\left(M F_{f}(0) ; A\right) \cong H^{n-1}\left(\mathcal{S}^{n-1} ; A\right) \cong A$. Therefore we have

$$
\mathcal{P} \mathcal{V}_{\mathbb{C}^{n}, z_{1}^{2}+\cdots+z_{n}^{2}}^{\bullet} \cong A_{\{0\}} \text {. }
$$

This isomorphism (2.12) is natural up to sign (unless the base ring $A$ has characteristic 2, in which case (2.12) is natural), as it depends on the choice of isomorphism $H^{n-1}\left(\mathcal{S}^{n-1}, A\right) \cong A$, which corresponds to an orientation for $\mathcal{S}^{n-1}$. This uncertainty of signs will be important in 55 -

We can also use Milnor fibres to compute the monodromy operator on $\mathcal{P} \mathcal{V}_{\mathbb{C}^{n}, z_{1}^{2}+\cdots+z_{n}^{2}}^{\bullet}$ There is a monodromy map $\mu_{f}: M F_{f}(0) \rightarrow M F_{f}(0)$, natural up to isotopy, which is the monodromy in the Milnor fibration of $f$ at 0 . Under the identification $M F_{f}(0) \cong T^{*} \mathcal{S}^{n-1}$ we may take $\mu_{f}$ to be the map d(-1): $T^{*} \mathcal{S}^{n-1} \rightarrow T^{*} \mathcal{S}^{n-1}$ induced by $-1: \mathcal{S}^{n-1} \rightarrow \mathcal{S}^{n-1}$ mapping $-1:\left(x_{1}, \ldots, x_{n}\right) \mapsto\left(-x_{1}, \ldots,-x_{n}\right)$. This multiplies orientations on $\mathcal{S}^{n-1}$ by $(-1)^{n}$. Thus, $\mu_{f *}: H^{n-1}\left(\mathcal{S}^{n-1}, A\right) \rightarrow H^{n-1}\left(\mathcal{S}^{n-1}, A\right)$ multiplies by $(-1)^{n}$.

By [14, Prop. 4.2.2], equation (2.11) identifies the action of the monodromy operator $\left.M_{\mathbb{C}^{n}, f}\right|_{\{0\}}$ on $\mathcal{P} \mathcal{V}_{\mathbb{C}^{n}, z_{1}^{2}+\cdots+z_{n}^{2}}^{\bullet}$ with the action of $\mu_{f *}$ on $H^{n-1}\left(\mathcal{S}^{n-1}, A\right)$. So $\left.M_{\mathbb{C}^{n}, f}\right|_{\{0\}}$ is multiplication by $(-1)^{n}$. Combining this with the sign change $(-1)^{\operatorname{dim} U}$ in (2.7) for $U=\mathbb{C}^{n}$ shows that the twisted monodromy is

$$
\tau_{\mathbb{C}^{n}, z_{1}^{2}+\cdots+z_{n}^{2}}=\mathrm{id}: \mathcal{P} \mathcal{V}_{\mathbb{C}^{n}, z_{1}^{2}+\cdots+z_{n}^{2}}^{\bullet} \longrightarrow \mathcal{P} \mathcal{V}_{\mathbb{C}^{n}, z_{1}^{2}+\cdots+z_{n}^{2}}^{\bullet}
$$

Equations (2.12)-(2.13) also hold for $n=0,1$, though (2.11) does not.

Note also that these results are compatible with the Thom-Sebastiani Theorem 2.13 and can be deduced from it and the case $n=1$.

We introduce some notation for pullbacks of $\mathcal{P} \mathcal{V}_{V, g}^{\bullet}$ by étale morphisms.

Definition 2.15. Let $U, V$ be smooth $\mathbb{C}$-schemes, $\Phi: U \rightarrow V$ an étale morphism, and $g: V \rightarrow \mathbb{C}$ a regular function. Write $f=g \circ \Phi: U \rightarrow \mathbb{C}$, and $X=\operatorname{Crit}(f), Y=\operatorname{Crit}(g)$ as $\mathbb{C}$-subschemes of $U, V$. Then $\left.\Phi\right|_{X}: X \rightarrow Y$ is étale. Define an isomorphism

$$
\mathcal{P} \mathcal{V}_{\Phi}:\left.\mathcal{P} \mathcal{V}_{U, f}^{\bullet} \longrightarrow \Phi\right|_{X} ^{*}\left(\mathcal{P} \mathcal{V}_{V, g}^{\bullet}\right) \quad \text { in } \operatorname{Perv}(X)
$$

by the commutative diagram for each $c \in f(X) \subseteq g(Y)$ :

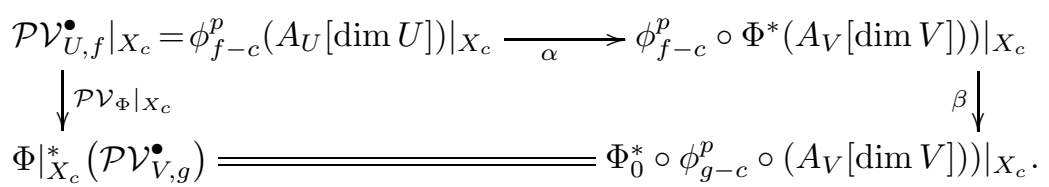

Here $\alpha$ is $\phi_{f-c}^{p}$ applied to the canonical isomorphism $A_{U} \rightarrow \Phi^{*}\left(A_{V}\right)$, noting that $\operatorname{dim} U=\operatorname{dim} V$ as $\Phi$ is étale, and $\beta$ is induced by (2.4). 
By naturality of the isomorphisms $\alpha, \beta$ in (2.15) we find the following commute, where $\sigma_{U, f}, \tau_{U, f}$ are as in (2.6)-2.7):

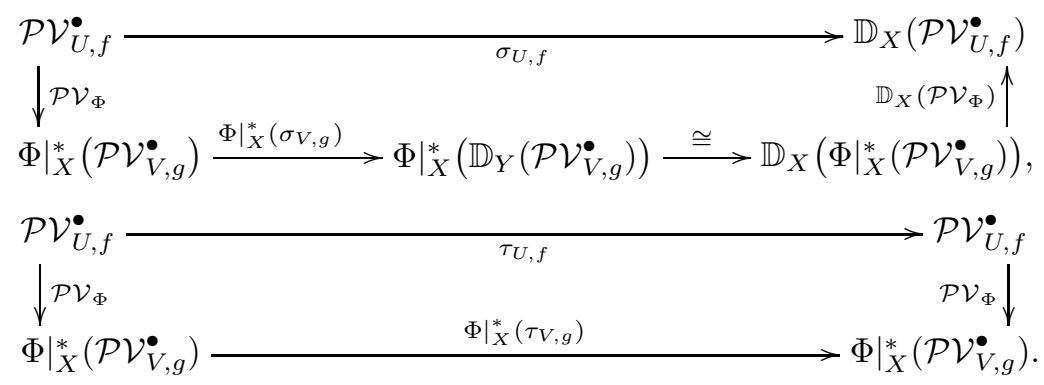

If $U=V, f=g$ and $\Phi=\operatorname{id}_{U}$ then $\mathcal{P} \mathcal{V}_{\operatorname{id}_{U}}=\operatorname{id}_{\mathcal{P} \mathcal{V}_{U, f}^{\bullet}}$.

If $W$ is another smooth $\mathbb{C}$-scheme, $\Psi: V \rightarrow W$ is étale, and $h: W \rightarrow \mathbb{C}$ is regular with $g=h \circ \Psi: V \rightarrow \mathbb{C}$, then composing (2.15) for $\Phi$ with $\left.\Phi\right|_{X_{c}} ^{*}$ of (2.15) for $\Psi$ shows that

$$
\mathcal{P} \mathcal{V}_{\Psi \circ \Phi}=\left.\Phi\right|_{X} ^{*}\left(\mathcal{P} \mathcal{V}_{\Psi}\right) \circ \mathcal{P} \mathcal{V}_{\Phi}:\left.\mathcal{P} \mathcal{V}_{U, f}^{\bullet} \longrightarrow(\Psi \circ \Phi)\right|_{X} ^{*}\left(\mathcal{P} \mathcal{V}_{W, h}^{\bullet}\right)
$$

That is, the isomorphisms $\mathcal{P} \mathcal{V}_{\Phi}$ are functorial.

Example 2.16. In Definition 2.15, set $U=V=\mathbb{C}^{n}$ and $f\left(z_{1}, \ldots, z_{n}\right)=$ $g\left(z_{1}, \ldots, z_{n}\right)=z_{1}^{2}+\cdots+z_{n}^{2}$, so that $Y=Z=\{0\} \subset \mathbb{C}^{n}$. Let $M \in \mathrm{O}(n, \mathbb{C})$ be an orthogonal matrix, so that $M: \mathbb{C}^{n} \rightarrow \mathbb{C}^{n}$ is an isomorphism with $f=g \circ M$ and $\left.M\right|_{\{0\}}=\operatorname{id}_{\{0\}}$. As $\left.M\right|_{Y}=\operatorname{id}_{Y}$, Definition 2.15 defines an isomorphism

$$
\mathcal{P} \mathcal{V}_{M}: \mathcal{P} \mathcal{V}_{\mathbb{C}^{n}, z_{1}^{2}+\cdots+z_{n}^{2}}^{\bullet} \mathcal{P} \mathcal{V}_{\mathbb{C}^{n}, z_{1}^{2}+\cdots+z_{n}^{2}}^{\bullet}
$$

Equation (2.11) describes $\mathcal{P} \mathcal{V}_{\mathbb{C}^{n}, z_{1}^{2}+\cdots+z_{n}^{2}}$ in terms of $M F_{f}(0) \cong T^{*} \mathcal{S}^{n-1}$. Now $\left.M\right|_{M F_{f}(0)}: M F_{f}(0) \rightarrow M F_{f}(0)$ multiplies orientations on $\mathcal{S}^{n-1}$ by $\operatorname{det} M$, so $\left(\left.M\right|_{M F_{f}(0)}\right)_{*}: H^{n-1}\left(M F_{f}(0) ; A\right) \rightarrow H^{n-1}\left(M F_{f}(0) ; A\right)$ is multiplication by $\operatorname{det} M$. Thus (2.11) implies that $\mathcal{P} \mathcal{V}_{M}$ in (2.19) is multiplication by $\operatorname{det} M= \pm 1$.

\subsection{Summary of the properties we use in this paper}

Since parts of $92.1-2.4$ do not work for the other kinds of perverse sheaves, $\mathscr{D}$ modules and mixed Hodge modules in $\$ 2.6$ - whe list what we will need for \6, to make it easy to check they are also valid in the settings of $\$ 2.6-22.10$.

(i) There should be an $A$-linear abelian category $\mathcal{P}(X)$ of $\mathcal{P}$-objects defined for each scheme or complex analytic space $X$, over a fixed, well-behaved base $\operatorname{ring} A$. We do not require $A$ to be a field.

(ii) There should be a Verdier duality functor $\mathbb{D}_{X}$ with $\mathbb{D}_{X} \circ \mathbb{D}_{X} \cong \mathrm{id}$, defined on a suitable subcategory of $\mathcal{P}$-objects on $X$ which includes the objects we are interested in. We do not need $\mathbb{D}_{X}$ to be defined on all objects in $\mathcal{P}(X)$. 
(iii) If $U$ is a smooth scheme or complex manifold, then there should be a canonical object $A_{U}[\operatorname{dim} U] \in \mathcal{P}(U)$, with a canonical isomorphism

$$
\mathbb{D}_{U}\left(A_{U}[\operatorname{dim} U]\right) \cong A_{U}[\operatorname{dim} U] .
$$

(iv) Let $f: X \hookrightarrow Y$ be a closed embedding of schemes or complex analytic spaces; this implies $f$ is proper. Then $f_{*}, f_{!}: \mathcal{P}(X) \rightarrow \mathcal{P}(Y)$ should exist, inducing an equivalence of categories $\mathcal{P}(X) \stackrel{\sim}{\longrightarrow} \mathcal{P}_{X}(Y)$ as in Theorem 2.6)(c), where $\mathcal{P}_{X}(Y)$ is the full subcategory of objects in $\mathcal{P}(Y)$ supported on $X$.

(v) Let $f: X \rightarrow Y$ be an étale morphism. Then the pullbacks $f^{*}, f^{!}: \mathcal{P}(Y) \rightarrow$ $\mathcal{P}(X)$ should exist. More generally, if $f: X \rightarrow Y$ is smooth of relative dimension $d$, then there should be pullbacks $f^{*}[d], f^{!}[-d]$ mapping $\mathcal{P}(Y) \rightarrow \mathcal{P}(X)$. If $X, Y$ are smooth, there should be a canonical isomorphism $f^{*}[d]\left(A_{Y}[\operatorname{dim} Y]\right) \cong A_{X}[\operatorname{dim} X]$. We do not need pullbacks to exist for general morphisms $f: X \rightarrow Y$, though see (xi) below.

(vi) An external tensor product $\stackrel{L}{\otimes}: \mathcal{P}(X) \times \mathcal{P}(Y) \rightarrow \mathcal{P}(X \times Y)$ should exist for all $X, Y$.

(vii) If $X$ is a scheme or complex analytic space, $P \rightarrow X$ a principal $\mathbb{Z} / 2 \mathbb{Z}$ bundle, and $\mathcal{Q}^{\bullet} \in \mathcal{P}(X)$, the twisted perverse sheaf $\mathcal{Q}^{\bullet} \otimes_{\mathbb{Z} / 2 \mathbb{Z}} P \in \mathcal{P}(X)$ should make sense as in Definition [2.9, and have the obvious functorial properties.

(viii) A vanishing cycle functor $\phi_{f}^{p}: \mathcal{P}(U) \rightarrow \mathcal{P}\left(U_{0}\right)$ and monodromy transformation $M_{U, f}: \phi_{f}^{p} \Rightarrow \phi_{f}^{p}$ in $\$ 2.4$ should exist for all smooth $U$ and regular/holomorphic $f: U \rightarrow \mathbb{A}^{1}$.

(ix) The functors $\mathbb{D}_{X}, f^{*}, f^{!}, f_{*}, f_{!}, \phi_{f}^{p}$ should satisfy the natural isomorphisms in Theorems 2.4 and 2.11 provided they exist. They should have the obvious compatibilities with $\stackrel{L}{\otimes}$, and restriction to (Zariski) open sets.

(x) There should be suitable subcategories of $\mathcal{P}$-objects which form a stack in the étale or complex analytic topologies, as in Theorem 2.7 In the algebraic case we only need Theorem 2.7(ii) to hold for Zariski open covers, not étale open covers.

(xi) Proposition 2.8 must hold. This involves pullbacks $j_{t}^{*}$ by a morphism $j_{t}: W_{t} \hookrightarrow W$ which is not étale or smooth, as in (v) above. But on objects we only consider $j_{t}^{*}\left(\pi_{X}^{*}\left(\mathcal{P}^{\bullet}\right)\right)=\left.\pi_{X}\right|_{W_{t}} ^{*}\left(\mathcal{P}^{\bullet}\right)$ which exists in $\mathcal{P}\left(W_{t}\right)$ by (v) as $\left.\pi_{X}\right|_{W_{t}}$ is étale, so $j_{t}^{*}$ is defined on the objects we need.

(xii) There should be a Thom-Sebastiani Theorem for $\mathcal{P}$-objects, so that the analogue of Theorem 2.13 holds.

Remark 2.17. The existence of a (bounded) derived category of $\mathcal{P}$-objects will not be assumed, or used, in this paper. On the other hand, in all the cases we consider, there will be a realization functor from the category of $\mathcal{P}$-objects to an appropriate category of constructible complexes, and the notation used above 
reflects this. So in (iii),(v) above, [1] does not stand for a shift in any derived category; the notation means a $\mathcal{P}$-object or morphism whose realization is the appropriate constructible object or morphism. See Remark 2.20 below.

\subsection{Perverse sheaves on complex analytic spaces}

Next we discuss perverse sheaves on complex analytic spaces, as in Dimca [14. The theory follows 22.1 2.4 replacing (smooth) $\mathbb{C}$-schemes by complex analytic spaces (complex manifolds), and regular functions by holomorphic functions.

Let $X$ be a complex analytic space, always assumed locally of finite type (that is, locally embeddable in $\mathbb{C}^{n}$ ). In the analogue of Definition 2.2, we fix a well-behaved commutative ring $A$, and consider sheaves of $A$-modules $\mathcal{S}$ on $X$ in the complex analytic topology. A sheaf $\mathcal{S}$ is called (analytically) constructible if all the stalks $\mathcal{S}_{x}$ for $x \in X$ are finite type $A$-modules, and there is a locally finite stratification $X=\coprod_{j \in J} X_{j}$ of $X$, where now $X_{j} \subseteq X$ for $j \in J$ are complex analytic subspaces of $X$, such that $\left.\mathcal{S}\right|_{X_{j}}$ is an $A$-local system for all $j \in J$.

Write $D(X)$ for the derived category of complexes $\mathcal{C}^{\bullet}$ of sheaves of $A$-modules on $X$, exactly as in $\$ 2.1$ and $D_{c}^{b}(X)$ for the full subcategory of bounded complexes $\mathcal{C}^{\bullet}$ in $D(X)$ whose cohomology sheaves $\mathcal{H}^{m}\left(\mathcal{C}^{\bullet}\right)$ are analytically constructible for all $m \in \mathbb{Z}$. Then $D(X), D_{c}^{b}(X)$ are triangulated categories.

When we wish to distinguish the complex algebraic and complex analytic theories, we will write $D_{c}^{b}(X)^{\text {alg }}, \operatorname{Perv}(X)^{\text {alg }}$ for the algebraic versions in 92.1 22.2 with $X$ a $\mathbb{C}$-scheme, and $D_{c}^{b}(X)^{\text {an }}, \operatorname{Perv}(X)^{\text {an }}$ for the analytic versions.

Here are the main differences between the material of $2.1-22.4$ for perverse sheaves on $\mathbb{C}$-schemes and on complex analytic spaces:

(a) If $f: X \rightarrow Y$ is an arbitrary morphism of $\mathbb{C}$-schemes, then as in $\$ 2.1$ the pushforwards $R f_{*}, R f_{!}: D(X) \rightarrow D(Y)$ also map $D_{c}^{b}(X)^{\text {alg }} \rightarrow D_{c}^{b}(Y)^{\text {alg }}$.

However, if $f: X \rightarrow Y$ is a morphism of complex analytic spaces, then $R f_{*}, R f_{!}: D(X) \rightarrow D(Y)$ need not map $D_{c}^{b}(X)^{\text {an }} \rightarrow D_{c}^{b}(Y)^{\text {an }}$ without extra assumptions on $f$, for example, if $f: X \rightarrow Y$ is proper.

(b) The analogue of Theorem 2.7 says that perverse sheaves on a complex analytic space $X$ form a stack in the complex analytic topology. This is proved in the subanalytic context in [27, Th. 10.2.9]; the analytic case follows upon noting that a sheaf is complex analytically constructible if and only if is locally at all points, as proved in [14, Prop. 4.1.13]. See also [21, Prop. 8.1.26].

The analogues of (i)-(xii) in $\$ 2.5$ work for complex analytic perverse sheaves, and so our main results hold in this context.

If $X$ is a $\mathbb{C}$-scheme, and $X^{\text {an }}$ the corresponding complex analytic space, then $D(X)$ in 2.1 for $X$ a $\mathbb{C}$-scheme coincides with $D\left(X^{\text {an }}\right)$ for $X^{\text {an }}$ a complex analytic space, and $D_{c}^{b}(X)^{\text {alg }} \subset D_{c}^{b}\left(X^{\text {an }}\right)^{\text {an }}, \operatorname{Perv}(X)^{\text {alg }} \subset \operatorname{Perv}\left(X^{\text {an }}\right)^{\text {an }}$ are

full subcategories, and the six functors $f^{*}, f^{!}, R f_{*}, R f_{!}, \mathcal{R H o m}, \stackrel{L}{\otimes}$ for $\mathbb{C}$-scheme morphisms $f: X \rightarrow Y$ agree in the algebraic and analytic cases. 


\section{$2.7 \mathscr{D}$-modules on $\mathbb{C}$-schemes and complex analytic spaces}

$\mathscr{D}$-modules on a smooth $\mathbb{C}$-scheme or smooth complex analytic space $X$ are sheaves of modules over a certain sheaf of rings of differential operators $\mathscr{D}_{X}$ on $X$. Some books on them are Borel et al. 8, Coutinho [12, and Hotta, Takeuchi and Tanisaki [21] in the $\mathbb{C}$-scheme case, and Björk [7] and Kashiwara [26] in the complex analytic case. For a singular complex $\mathbb{C}$-scheme or complex analytic space $X$, the definition of a well-behaved category of $\mathscr{D}$-modules is given by Saito [48, via locally embedding $X$ into a smooth scheme or space.

The analogue of perverse sheaves on $X$ are called regular holonomic $\mathscr{D}$ modules, which form an abelian category $\operatorname{Mod}_{\mathrm{rh}}\left(\mathscr{D}_{X}\right)$, the heart in the derived category $D_{\mathrm{rh}}^{b}\left(\operatorname{Mod}\left(\mathscr{D}_{X}\right)\right)$ of bounded complexes of $\mathscr{D}_{X}$-modules with regular holonomic cohomology modules. The whole package of $92.1-\sqrt{2.4}$ works for $\mathscr{D}$ modules. Our next theorem is known as the Riemann-Hilbert correspondence [7, $\S$ V.5], [21, Th. 7.2.1], see Borel [8, §14.4] for $\mathbb{C}$-schemes, Kashiwara [25] for complex manifolds, and Saito [48, §6] for complex analytic spaces, and also Maisonobe and Mekhbout 34.

Theorem 2.18. Let $X$ be $a \mathbb{C}$-scheme or complex analytic space. Then there is a de Rham functor $\mathrm{DR}: D_{\mathrm{rh}}^{b}\left(\operatorname{Mod}\left(\mathscr{D}_{X}\right)\right) \stackrel{\sim}{\longrightarrow} D_{c}^{b}(X, \mathbb{C})$, which is an equivalence of categories, restricts to an equivalence $\operatorname{Mod}_{\mathrm{rh}}\left(\mathscr{D}_{X}\right) \stackrel{\sim}{\longrightarrow} \operatorname{Perv}(X, \mathbb{C})$, and commutes with $f^{*}, f^{!}, R f_{*}, R f_{!}, \mathcal{R H o m}, \stackrel{L}{\otimes}$, and also with $\psi_{f}^{p}, \phi_{f}^{p}$ for $X$ smooth. Here $D_{c}^{b}(X, \mathbb{C}), \operatorname{Perv}(X, \mathbb{C})$ are constructible complexes and perverse sheaves over the base ring $A=\mathbb{C}$.

Because of the Riemann-Hilbert correspondence, all our results on perverse sheaves of vanishing cycles on $\mathbb{C}$-schemes and complex analytic spaces in $\sqrt{3}-96$ over a well-behaved base ring $A$, translate immediately when $A=\mathbb{C}$ to the corresponding results for $\mathscr{D}$-modules of vanishing cycles, with no extra work.

\subsection{Mixed Hodge modules: basics}

We write this section in the minimal generality needed for our applications. The statements made work equally well in the category of (algebraic) $\mathbb{C}$-schemes and the category of complex analytic spaces. By space, we will mean an object in either of these categories. The theory of mixed Hodge modules works with reduced spaces; should a space $X$ be non-reduced, the following constructions are taken by definition on its reduction.

For a space $X$, let $\operatorname{HM}(X)$ denote Saito's category [45] of polarizable pure Hodge modules, (locally) a direct sum of subcategories $\operatorname{HM}(X)^{w}$ of pure Hodge modules of fixed weight $w$. On a smooth $X$, a pure Hodge module $M^{\bullet}$ consists of a triple of data: a filtered holonomic $\mathscr{D}$-module $(M, F)$, a $\mathbb{Q}$-perverse sheaf, and a comparison map identifying the former with the complexification of the latter under the Riemann-Hilbert correspondence; see [45, §5.1.1, p. 952] and [47, §4]. This triple has to satisfy many other properties; in particular, the underlying holonomic $\mathscr{D}$-module is automatically regular, and algebraic Hodge modules are asked to be extendable to an algebraic compactification. Thus 
there is a forgetful functor $\operatorname{HM}(X) \rightarrow \operatorname{Mod}_{\mathrm{rh}}\left(\mathscr{D}_{X}\right)$ from Hodge modules to regular holonomic (algebraic) $\mathscr{D}$-modules. Hodge modules on singular spaces are defined, similarly to $\mathscr{D}$-modules, via embeddings into smooth varieties; see Saito [4] and also Maxim, Saito and Schürmann [39, §1.8].

There is a duality functor $\mathbb{D}_{X}^{H}: \operatorname{HM}(X) \rightarrow \operatorname{HM}(X)$. Pure Hodge modules also admit a Tate twist functor $M^{\bullet} \mapsto M^{\bullet}(1)$, see [45, §5.1.3, p. 952]. This functor shifts the filtration and rotates the rational structure on the underlying perverse sheaf: the $\mathscr{D}$-module filtration $(M, F)$ is shifted to $(M, F[n])$ with $(F[n])_{i}=F_{i-n}$; the underlying perverse sheaf is tensored by $\mathbb{Z}(n)=(2 \pi i)^{n} \mathbb{Z} \subset$ $\mathbb{C}$, as in [45, (2.0.2), p. 876].

A polarization of weight $w$ on a pure Hodge module $M^{\bullet} \in \operatorname{HM}(X)^{w}$ is a morphism of pure Hodge modules

$$
\sigma: M^{\bullet} \longrightarrow \mathbb{D}_{X}^{H}\left(M^{\bullet}\right)(-w)
$$

satisfying the extra conditions using vanishing cycles described on [45, (5.1.6.2) on p. 956 and (5.2.10.2) on p. 968], as well as the condition that on points it should correspond to the classical notion of a polarization of a pure Hodge structure (including positive definiteness).

Next, let $\operatorname{MHM}(X)$ denote the category of graded polarizable mixed Hodge modules 45, 47. A graded polarizable mixed Hodge module carries a functorial weight filtration $W$, with graded pieces being polarizable pure Hodge modules, see [45, §5.2.10, p. 967-8]. The forgetful functor rat : $\operatorname{MHM}(X) \rightarrow \operatorname{Perv}(X)$ to the appropriate category of perverse $\mathbb{Q}$-sheaves on $X$ is faithful and exact; faithfulness in particular means that a morphism in $\operatorname{MHM}(X)$ is uniquely determined by the underlying morphism of perverse sheaves. The Tate twist functor extends to $\operatorname{MHM}(X)$; under this functor, the weight filtration $W$ of the mixed Hodge module is changed to $W[2 n]$ with $W[2 n]_{i}=W_{i+2 n}$ as on [45, p. 855]. The duality functor $\mathbb{D}_{X}^{H}$ also extends to $\operatorname{MHM}(X)$ and is compatible with Verdier duality on the perverse realization. There is also a forgetful functor $\operatorname{MHM}(X) \rightarrow \operatorname{Mod}_{\mathrm{rh}}\left(\mathscr{D}_{X}\right)$ to regular holonomic $\mathscr{D}$-modules, even for singular spaces.

Theorem 2.19. The categories of graded polarizable mixed Hodge modules have the following properties:

(i) By [47, Th. 3.9, p. 288], the category of mixed Hodge modules for $X$ a point is canonically equivalent to Deligne's category of graded polarizable mixed Hodge structures.

(ii) For a smooth space $U$, we have a canonical object of weight $\operatorname{dim} U$

$$
\mathbb{Q}_{U}^{H}[\operatorname{dim} U] \in \operatorname{HM}(U) \subset \operatorname{MHM}(U),
$$

which by [45, Prop. 5.2.16, p. 971] possesses a canonical polarization $\sigma$ : $\mathbb{Q}_{U}^{H}[\operatorname{dim} U] \rightarrow \mathbb{D}_{U}^{H} \mathbb{Q}_{U}^{H}[\operatorname{dim} U](-\operatorname{dim} U)$.

(iii) For an open inclusion $f: Y \hookrightarrow X$ of spaces, there is a pullback functor $f^{*}=f^{!}: \operatorname{MHM}(X) \rightarrow \operatorname{MHM}(Y)$. More generally, by [47, Prop. 2.19, 
p. 258], for an arbitrary morphism $f: Y \rightarrow X$, there exist cohomological pullback functors $L^{j} f^{*}, L^{j} f^{!}: \operatorname{MHM}(X) \rightarrow \operatorname{MHM}(Y)$ compatible with (perverse) cohomological pullback on the perverse sheaf level.

(iv) For a closed embedding $i: X \hookrightarrow Y$, there is a pushforward functor

$$
i_{*}=i_{!}: \operatorname{MHM}(X) \longrightarrow \operatorname{MHM}(Y)
$$

whose essential image is the full subcategory $\operatorname{MHM}_{X}(Y)$ of objects in $\operatorname{MHM}(Y)$ supported on $X$. Its inverse is $i^{*}=i^{!}: \operatorname{MHM}_{X}(Y) \rightarrow \operatorname{MHM}(X)$. More generally, by [45, Th. 5.3.1, p. 977] and [47, Th. 2.14, p. 252], for a projective map $f: X \rightarrow Y$ there are cohomological pushforward functors

$$
R^{j} f_{*}: \operatorname{MHM}(X) \longrightarrow \operatorname{MHM}(Y) .
$$

(v) There is an external tensor product functor

$$
\stackrel{L}{\otimes}: \operatorname{MHM}(X) \times \operatorname{MHM}(Y) \longrightarrow \operatorname{MHM}(X \times Y),
$$

which is compatible with duality in the sense that for $M^{\bullet} \in \operatorname{MHM}(X)$ and $N^{\bullet} \in \operatorname{MHM}(Y)$, there is a natural isomorphism

$$
\mathbb{D}_{X}^{H} M^{\bullet} \stackrel{L}{\otimes} \mathbb{D}_{Y}^{H} N^{\bullet} \cong \mathbb{D}_{X \times Y}^{H}\left(M^{\bullet} \stackrel{L}{\otimes} N^{\bullet}\right) .
$$

Remark 2.20. We will not need to use any derived category $D^{\text {? }} \operatorname{MHM}(X)$ of mixed Hodge modules in this paper, which is just as well since on singular analytic $X$, the appropriate boundedness conditions do not appear to be well understood, and the general pullback and pushforward functors of Theorem 2.19(iii),(iv) are not known to exist as derived functors outside of the algebraic context of [47, §4]. Hence, in part (ii) above, [1] does not stand for a shift in the derived category; $\mathbb{Q}_{U}^{H}[\operatorname{dim} U]$ just denotes a mixed Hodge module whose realization is the perverse sheaf $\mathbb{Q}_{U}[\operatorname{dim} U]$ on $U$. Compare Remark 2.17 above.

Using the functors above, we can now define the twist of a mixed Hodge module by a principal $\mathbb{Z} / 2 \mathbb{Z}$-bundle. In the setup of Definition 2.9 , given a $\mathbb{Z} / 2 \mathbb{Z}$-bundle $\pi: P \rightarrow X$, and an object $M^{\bullet} \in \operatorname{MHM}(X)$ on a space $X$, we have a natural map $M^{\bullet} \rightarrow \pi_{*} \pi^{*} M^{\bullet}$, which is an injection by faithfulness of the realization functor and the fact that it is an injection on the perverse sheaf level. The quotient object will be denoted, by abuse of notation, by $M^{\bullet} \otimes_{\mathbb{Z} / 2 \mathbb{Z}} P$ in $\operatorname{MHM}(X)$.

\subsection{Monodromic mixed Hodge modules}

To discuss nearby and vanishing cycle functors in a way consistent with monodromy, we need an extension of the category of mixed Hodge modules. For a space $X$, following Saito [49, $\S 4.2]$ denote by $\operatorname{MHM}\left(X ; T_{s}, N\right)$ the category of mixed Hodge modules $M^{\bullet}$ on $X$ with commuting actions of a finite order operator $T_{s}: M^{\bullet} \rightarrow M^{\bullet}$ and a locally nilpotent operator $N: M^{\bullet} \rightarrow M^{\bullet}(-1)$. There 
is an embedding of categories $\operatorname{MHM}(X) \rightarrow \operatorname{MHM}\left(X ; T_{s}, N\right)$ defined by setting $T=\mathrm{id}$ and $N=0$. As proved by [49, (4.6.2)], the category $\operatorname{MHM}\left(X ; T_{s}, N\right)$ is equivalent to the category $\operatorname{MHM}(X \times \mathbb{C})_{\text {mon,! }}$ of monodromic mixed Hodge modules on $X \times \mathbb{C}^{*}$ extended by zero to $X \times \mathbb{C}$; compare also [33, $\$ 4.2$ ].

Every object $M^{\bullet} \in \operatorname{MHM}\left(X ; T_{s}, N\right)$ decomposes into a direct sum $M^{\bullet}=$ $M_{1}^{\bullet} \oplus M_{\neq 1}^{\bullet}$ of the $T_{s}$-invariant part and its $T_{s}$-equivariant complement. The Tate twist, and appropriate cohomological pullback and pushforward functors continue to exist. There is a duality functor

$$
\mathbb{D}_{X}^{T}: \operatorname{MHM}\left(X ; T_{s}, N\right) \longrightarrow \operatorname{MHM}\left(X ; T_{s}, N\right)
$$

defined by

$$
\mathbb{D}_{X}^{T}\left(M^{\bullet}\right)=\mathbb{D}_{X}^{H}\left(M_{1}^{\bullet}\right) \oplus \mathbb{D}_{T}^{H}\left(M_{\neq 1}^{\bullet}\right)(1),
$$

equipped with the finite-order operator $\mathbb{D}_{X}\left(T_{s}\right)^{-1}$ and the nilpotent operator $-\mathbb{D}_{X}(N)$. This duality functor still satisfies $\mathbb{D}_{X}^{T} \circ \mathbb{D}_{X}^{T}=\mathrm{id}$.

Saito [4, §5.1] also defines an external tensor product

$$
\stackrel{T}{\otimes}: \operatorname{MHM}\left(X_{1} ; T_{s}, N\right) \times \operatorname{MHM}\left(X_{2} ; T_{s}, N\right) \longrightarrow \operatorname{MHM}\left(X_{1} \times X_{2} ; T_{s}, N\right) .
$$

defined on the monodromic category as follows. The addition map on fibres

$$
\pi:\left(X_{1} \times \mathbb{C}\right) \times\left(X_{2} \times \mathbb{C}\right) \rightarrow\left(X_{1} \times X_{2}\right) \times \mathbb{C}
$$

induces the additive convolution

$\pi_{*}(-\otimes-): \operatorname{MHM}\left(X_{1} \times \mathbb{C}\right)_{\operatorname{mon}, !} \times \operatorname{MHM}\left(X_{2} \times \mathbb{C}\right)_{\text {mon, },} \rightarrow \operatorname{MHM}\left(X_{1} \times x_{2} \times \mathbb{C}\right)_{\text {mon }, !}$

One can translate this external tensor product $\stackrel{T}{\otimes}^{\mathrm{T}}$ to the $\operatorname{MHM}\left(X ; T_{s}, N\right)$ defined by concrete data $\left(M^{\bullet}, T_{s}, N\right)$. On the underlying $\mathscr{D}$-modules and perverse sheaves, it is just the usual product $\otimes$. The operators are defined by $T_{s}=T_{s} \otimes T_{s}$ and $N=N \otimes$ id + id $\otimes N$. However, the Hodge and weight filtrations on the underlying $\mathscr{D}$-modules and perverse sheaves are shifted using the finite order endomorphisms $T_{s}$; for details, see [49, (5.1.1)-(5.1.2)]. Note that as a consequence of these definitions, the forgetful functors $\operatorname{MHM}\left(-; T_{s}, N\right) \rightarrow \operatorname{MHM}(-)$ do not map $\stackrel{T}{\otimes}$ to $\stackrel{L}{\otimes}$. Twisted duality and the twisted tensor product commute in the sense that given $M^{\bullet} \in \operatorname{MHM}\left(X ; T_{s}, N\right)$ and $N^{\bullet} \in \operatorname{MHM}\left(Y ; T_{s}, N\right)$, we have a natural isomorphism in $\operatorname{MHM}\left(X \times Y ; T_{s}, N\right)$

$$
\mathbb{D}_{X}^{T}\left(M^{\bullet}\right) \stackrel{T}{\otimes} \mathbb{D}_{Y}^{T}\left(N^{\bullet}\right) \cong \mathbb{D}_{X \times Y}^{T}\left(M^{\bullet} \stackrel{T}{\otimes} N^{\bullet}\right) .
$$

For an object $M^{\bullet} \in \operatorname{MHM}\left(X ; T_{s}, N\right)$ whose weight filtration is a (suitable shifted) monodromy filtration of the nilpotent morphism $N$, there is a stronger notion of polarization which will be useful for us. A strong polarization of weight $w$ of such an object $M^{\bullet}$ is a morphism $\sigma: M^{\bullet} \rightarrow \mathbb{D}_{X}^{T}\left(M^{\bullet}\right)(-w)$ in $\operatorname{MHM}(X)$, compatible with $T_{s}$ and $N$, such that $\sigma$ defines polarizations on the $N$-primitive parts of $M^{\bullet}$, compatible with Hodge filtrations; for precise conditions, see [45], 
p. 855]. A polarization on a pure Hodge module is a strong polarization (with $N=0$ ); a strongly polarized mixed Hodge module is graded polarizable. The partial twist in the definition of $\mathbb{D}_{X}^{T}$ implies that $M^{\bullet}$ is of weight $w$ if and only if $M_{1}^{\bullet}$, respectively $M_{\neq 1}^{\bullet}$ are of weights $w, w-1$ in the sense of [45, p. 855].

Given strongly polarized mixed Hodge modules $M_{i}^{\bullet} \in \operatorname{MHM}\left(X_{i} ; T_{s}, N\right)$ of weight $w_{i}$ for $i=1,2$, polarized by $\sigma_{i}: M_{i}^{\bullet} \rightarrow \mathbb{D}_{X_{i}}^{T}\left(M_{i}^{\bullet}\right)\left(-w_{i}\right)$, there is an induced morphism $\sigma$ in a commutative diagram

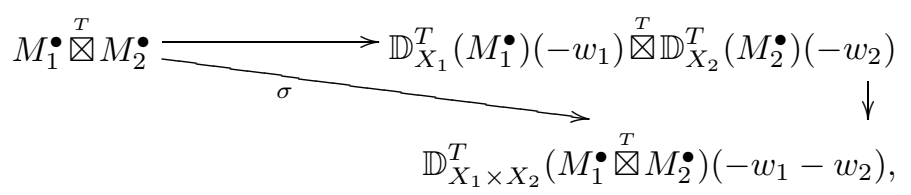

where the top map is $\sigma_{1}{ }^{T} \sigma_{2}$ and the right is the isomorphism (2.20). In general, it is not clear whether this morphism is a strong polarization of the tensor product $M_{1}^{\bullet}{ }^{T} M_{2}^{\bullet}$; this result is not available in the literature. However, in this paper we only use this construction in cases where one of the monodromic mixed Hodge modules is essentially trivial, living on $X_{1}=$ pt with $N=0$, in which case it is easy to check that the resulting $\sigma$ is a strong polarization.

Note also that if $M^{\bullet}$ is strongly polarized by $\sigma: M^{\bullet} \rightarrow \mathbb{D}_{X}^{T}\left(M^{\bullet}\right)(-w)$, then its Tate twist is also strongly polarized by the composition

$$
M \bullet(1) \stackrel{\sigma(1)}{\longrightarrow} \mathbb{D}_{X}^{T}\left(M^{\bullet}\right)(-w+1) \stackrel{\sim}{\longrightarrow} \mathbb{D}_{X}^{T}(M \bullet(1))(-w+2) .
$$

The notion of strong polarization leads to gluing, in the following way.

Theorem 2.21. Let $X=\bigcup_{i} U_{i}$ be an open cover of a space $X$, in any of the Zariski, étale or complex analytic topologies. Then:

(i) Suppose we are given mixed Hodge modules $M^{\bullet}, N^{\bullet} \in \operatorname{MHM}(X)$, with morphisms $f_{i}:\left.\left.M^{\bullet}\right|_{U_{i}} \rightarrow N^{\bullet}\right|_{U_{i}}$ in $\operatorname{MHM}\left(U_{i}\right)$ which agree on overlaps $U_{i j}$. Then there is a unique $f \in \operatorname{Hom}_{\operatorname{MHM}(X)}\left(M^{\bullet}, N^{\bullet}\right)$ with $\left.f\right|_{U_{i}}=f_{i}$.

(ii) Suppose we are given mixed Hodge modules $M_{i}^{\bullet} \in \operatorname{MHM}\left(U_{i} ; T_{s}, N\right)$, each equipped with a strong polarization $\sigma_{i}$. Suppose also that we are given isomorphisms $\alpha_{i j}:\left.\left.M_{i}^{\bullet}\right|_{U_{i j}} \rightarrow M_{j}^{\bullet}\right|_{U_{i j}}$ on intersections, commuting with the restrictions of the maps $T_{s i}, N_{i}$ and $\sigma_{i}$, with $\left.\left.\alpha_{j k}\right|_{U_{i j k}} \circ \alpha_{i j}\right|_{U_{i j k}}=\left.\alpha_{i k}\right|_{U_{i j k}}$ on triple intersections. Then there is a strongly polarized mixed Hodge module $M^{\bullet} \in \operatorname{MHM}\left(X ; T_{s}, N\right)$, restricting to $M_{i}^{\bullet}$ on $U_{i}$.

Proof. To prove (i), it is enough to note that the $f_{i}$ glue on the perverse sheaf and $\mathscr{D}$-module levels, respecting filtrations.

To prove (ii), we begin with the case of pure Hodge modules of fixed weight $w$. The data of a pure Hodge module consists of a pair of a filtered holonomic $\mathscr{D}$-module and a $\mathbb{Q}$-perverse sheaf, with an identification of the former with the complexification of the latter under the Riemann-Hilbert correspondence. Since 
both filtered holonomic $\mathscr{D}$-modules and $\mathbb{Q}$-perverse sheaves form stacks (both in the algebraic and the analytic case), this data glues over $X$. As for the (strong) polarization, $T_{s i}=\mathrm{id}$ and $N_{i}=0$ glue to $T_{s}=\mathrm{id}$ and $N=0$, whereas the map $\sigma$ on the perverse sheaf level glues from the maps $\sigma_{i}$ once again from the stack property (now for morphisms) of perverse sheaves.

The conditions [45, §5.1.6, p. 955] which make such a pair a pure Hodge module come from local conditions as well as conditions on vanishing cycles; the latter glue by induction on the dimension. So strongly polarized pure Hodge modules form a stack. The case of mixed Hodge modules is similar: we need to glue filtrations and polarizations, as well as the maps $T_{s i}, N_{i}$ and $\sigma_{i}$, first on the level of perverse sheaves, and then checking the axioms, which are local or follow by induction.

Remark 2.22. Given a projective $\mathbb{C}$-scheme $X$, and a polarizable mixed Hodge module $M^{\bullet} \in \operatorname{MHM}(X)$ on it, the second part of Theorem 2.19(iv) applied to $f: X \rightarrow$ pt shows that the hypercohomology $\mathbb{H}^{*}\left(X, M^{\bullet}\right)$ carries a mixed Hodge structure. In particular, it carries a weight filtration and therefore has a weight polynomial, which will be useful in refinements of Donaldson-Thomas theory, see the discussion in Remark 6.14 below. So we need to glue polarizable objects from local data. On the other hand, graded polarizable mixed Hodge modules may fail to form a stack in the analytic category unless the polarizations glue. This is the reason for using the stronger form of polarization, which allows for gluing as shown above.

\subsection{Mixed Hodge modules of vanishing cycles}

By Saito's work [45 47], for a regular function $f: U \rightarrow \mathbb{C}$ on a smooth space $U$, the perverse nearby and vanishing cycle functors $\psi_{f}^{p}, \phi_{f}^{p}$ defined on perverse sheaves in $\$ 2.3$ lift to functors

$$
\psi_{f}^{H}, \phi_{f}^{H}: \operatorname{MHM}(U) \longrightarrow \operatorname{MHM}\left(U_{0} ; T_{s}, N\right),
$$

where $U_{0}=f^{-1}(0)$. The actions of the finite order and nilpotent operators $T_{s}, N$ are given by the semisimple part of the monodromy operator, and the logarithm of its unipotent part. The analogue

$$
\phi_{f}^{H} \circ \mathbb{D}_{U}^{T} \cong \mathbb{D}_{U_{0}}^{T} \circ \phi_{f}^{H}
$$

of Theorem 2.11(iv) is proved in 46]; to make this isomorphism work is the reason for the the twist in the definition of $\mathbb{D}_{U}^{T}$. Note also that [46, Th. 1.6] fits with the convention that $T_{s}$ and $N$ are defined on dual objects as $\mathbb{D}_{U}\left(T_{s}\right)^{-1}$ and $\mathbb{D}_{U}(N)$, respectively.

By [45, 55.2$]$, if $M^{\bullet} \in \mathrm{HM}(U)$ is a pure Hodge module, then a polarization of $M^{\bullet}$ induces a strong polarization on the (mixed) Hodge module of vanishing cycles $\phi_{f}^{H}\left(M^{\bullet}\right)$, of the same weight. In particular, if $M^{\bullet}=\mathbb{Q}_{U}^{H}[\operatorname{dim} U]$ is the canonical object with its canonical polarization from Theorem [2.19)(ii), then 
$\phi_{f}^{H}\left(\mathbb{Q}_{U}^{H}[\operatorname{dim} U]\right) \in \operatorname{MHM}\left(\operatorname{Crit}(f) ; T_{s}, N\right)$ is a strongly polarized mixed Hodge module on the critical locus of $f$, with polarization

$$
\sigma: \phi_{f}^{H}\left(\mathbb{Q}_{U}^{H}[\operatorname{dim} U]\right) \longrightarrow \mathbb{D}_{\operatorname{Crit}(f)}^{T} \circ \phi_{f}^{H}\left(\mathbb{Q}_{U}^{H}[\operatorname{dim} U]\right)(-\operatorname{dim} U) .
$$

Example 2.23. Define $f: \mathbb{C} \rightarrow \mathbb{C}$ by $f(z)=z^{2}$. Then $\operatorname{Crit}(f)=\{0\}$, and we obtain an object $\phi_{f}^{H}\left(\mathbb{Q}_{\mathbb{C}}^{H}[1]\right)$ in $\operatorname{MHM}\left(\mathrm{pt} ; T_{s}, N\right)$, a one-dimensional polarized mixed Hodge structure with monodromy acting by $T_{s}=-\mathrm{id}$ and $N=0$. For $g: \mathbb{C}^{2} \rightarrow \mathbb{C}$ given by $g\left(z_{1}, z_{2}\right)=z_{1}^{2}+z_{2}^{2}$, it is well known that

$$
\phi_{z_{1}^{2}+z_{2}^{2}}^{H}\left(\mathbb{Q}_{\mathbb{C}^{2}}^{H}[2]\right) \cong \mathbb{Q}(-1)
$$

with trivial monodromy action. Applying the Thom-Sebastiani formula for mixed Hodge modules [49, Th. 5.4], we see that

$$
\phi_{z^{2}}^{H}\left(\mathbb{Q}_{\mathbb{C}}^{H}[1]\right) \stackrel{T}{\otimes} \phi_{z^{2}}^{H}\left(\mathbb{Q}_{\mathbb{C}}^{H}[1]\right) \cong \mathbb{Q}(-1)
$$

in the category $\operatorname{MHM}\left(\mathrm{pt} ; T_{s}, N\right)$. The objects $\mathbb{Q}(1)$ and $\mathbb{Q}(-1)$ thus admit square roots under $\stackrel{T}{\otimes}$ in this category, which we will denote by $\mathbb{Q}\left(\frac{1}{2}\right)$ and $\mathbb{Q}\left(-\frac{1}{2}\right)$, where

$$
\phi_{z^{2}}^{H}\left(\mathbb{Q}_{\mathbb{C}}^{H}[1]\right)=\mathbb{Q}\left(-\frac{1}{2}\right) .
$$

More explicitly, we have

$$
\mathbb{Q}\left(-\frac{1}{2}\right)=(\mathbb{Q}(0),-\mathrm{id}, 0)
$$

and

$$
\mathbb{Q}\left(\frac{1}{2}\right)=(\mathbb{Q}(1),-\mathrm{id}, 0) .
$$

Define an object $\mathbb{Q}\left(\frac{n}{2}\right) \in \operatorname{MHM}\left(\mathrm{pt} ; T_{s}, N\right)$ for each $n \in \mathbb{Z}$ by $\mathbb{Q}\left(\frac{n}{2}\right)=\mathbb{Q}\left(\frac{1}{2}\right)^{\mathbb{\boxplus}^{n}}$ for $n \geqslant 0$, and $\mathbb{Q}\left(\frac{n}{2}\right)=\mathbb{Q}\left(-\frac{1}{2}\right)^{\frac{T}{\otimes^{-n}}}$ for $n<0$. For any space $X$ with structure morphism $\pi: X \rightarrow$ pt, and any $M^{\bullet} \in D^{b} \operatorname{MHM}\left(X ; T_{s}, N\right)$, we define the $\frac{n}{2}$ twist of $M^{\bullet}$ to be $M^{\bullet}\left(\frac{n}{2}\right)=M^{\bullet} \stackrel{T}{\otimes}\left(\mathbb{Q}\left(\frac{n}{2}\right)\right)$. If $M^{\bullet}$ is strongly polarized, then this tensor product is also strongly polarized by the tensor polarization by our comments above.

Let $U$ be a smooth space, $f: U \rightarrow \mathbb{C}$ a regular function, and $X=\operatorname{Crit}(f)$ its critical locus, as a subspace of $U$. The perverse sheaf of vanishing cycles $\mathcal{P} \mathcal{V}_{U, f}^{\bullet} \in \operatorname{Perv}(X)$ from $\$ 2.4$ has a lift to a mixed Hodge module $\mathcal{H V}_{U, f}^{\bullet}$ in $\operatorname{MHM}\left(X ; T_{s}, N\right)$, defined for each $c \in f(X)$ by

$$
\left.\mathcal{H} \mathcal{V}_{U, f}^{\bullet}\right|_{X_{c}}=\left.\phi_{f-c}^{H}\left(\mathbb{Q}_{U}^{H}[\operatorname{dim} U]\right)\right|_{X_{c}}\left(\frac{1}{2} \operatorname{dim} U\right) \in \operatorname{MHM}\left(X_{c} ; T_{s}, N\right) .
$$

This mixed Hodge module inherits a strong polarization of weight 0 (compare (2.21) and (2.22)

$$
\sigma_{U, f}^{H}: \mathcal{H} \mathcal{V}_{U, f}^{\bullet} \longrightarrow \mathbb{D}_{X}^{T}\left(\mathcal{H} \mathcal{V}_{U, f}^{\bullet}\right)
$$


The twist $\left(\frac{1}{2} \operatorname{dim} U\right)$ in (2.24), using the notation of Example 2.23, is included for the same reason as the $(-1)^{\operatorname{dim} U}$ in the definition (2.7) of $\tau_{U, f}$. It makes $\mathcal{H} \mathcal{V}_{U, f}^{\bullet}$ act naturally under transformations which change dimension - without it, the mixed Hodge module version of (5.15) below would have to include a twist $\left(\frac{1}{2} n\right)$ for $n=\operatorname{dim} V-\operatorname{dim} U$. Then $\mathcal{H} \mathcal{V}_{U, f}^{\bullet}, T_{s}: \mathcal{H V}_{U, f}^{\bullet} \rightarrow \mathcal{H V}_{U, f}^{\bullet}, N$ : $\mathcal{H} \mathcal{V}_{U, f}^{\bullet} \rightarrow \mathcal{H} \mathcal{V}_{U, f}^{\bullet}(-1)$ and $\sigma_{U, f}^{H}: \mathcal{H} \mathcal{V}_{U, f}^{\bullet} \rightarrow \mathbb{D}_{X}^{T}\left(\mathcal{H} \mathcal{V}_{U, f}^{\bullet}\right)$ are related to $\mathcal{P} \mathcal{V}_{U, f}^{\bullet}$ $\tau_{U, f}: \mathcal{P} \mathcal{V}_{U, f}^{\bullet} \rightarrow \mathcal{P} \mathcal{V}_{U, f}^{\bullet}$ and $\sigma_{U, f}: \mathcal{P} \mathcal{V}_{U, f}^{\bullet} \cong \mathbb{D}_{X}\left(\mathcal{P} \mathcal{V}_{U, f}^{\bullet}\right)$ in $\llbracket 2.4$ by

$$
\mathcal{P} \mathcal{V}_{U, f}^{\bullet}=\operatorname{rat}\left(\mathcal{H} \mathcal{V}_{U, f}^{\bullet}\right), \tau_{U, f}=\operatorname{rat}\left(T_{s}\right) \circ \exp (2 \pi i \operatorname{rat}(N)), \quad \sigma_{U, f}=\operatorname{rat}\left(\sigma_{U, f}^{H}\right)
$$

for the last statement, see Proposition A.1 in the Appendix.

The following Thom-Sebastiani type result is the analogue of Theorem 2.13 .

Theorem 2.24. Let $U, V$ be smooth spaces and $f: U \rightarrow \mathbb{C}, g: V \rightarrow \mathbb{C}$ be regular functions, so that $f \boxplus g: U \times V \rightarrow \mathbb{C}$ is given by $(f \boxplus g)(u, v):=$ $f(u)+g(v)$. Set $X=\operatorname{Crit}(f)$ and $Y=\operatorname{Crit}(g)$ as subspaces of $U, V$, so that $\operatorname{Crit}(f \boxplus g)=X \times Y$. Then there is a natural isomorphism

$$
\mathcal{T} \mathcal{S}_{U, f, V, g}^{H}: \mathcal{H} \mathcal{V}_{U \times V, f \boxplus g}^{\bullet} \stackrel{\cong}{\longrightarrow} \mathcal{V}_{U, f}^{\bullet}{ }^{T} \mathcal{H} \mathcal{V}_{V, g}^{\bullet} \text { in } \operatorname{MHM}\left(X \times Y ; T_{s}, N\right),
$$

so that the following diagram commutes:

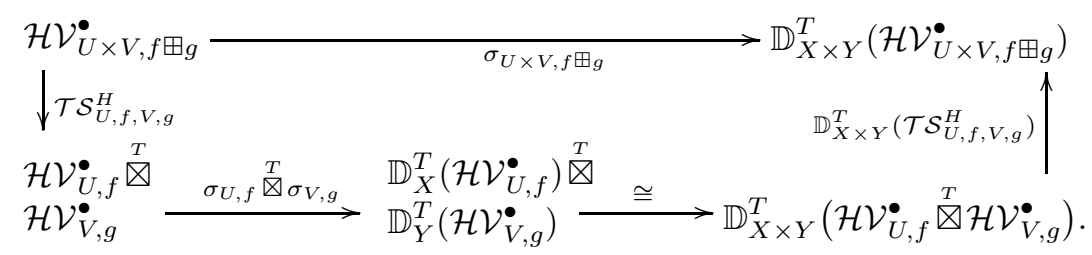

Proof. The existence of the isomorphism (2.26) follows from the Thom-Sebastiani Theorem for mixed Hodge modules due to Saito [49, Th. 5.4], applied to $\mathcal{H} \mathcal{V}_{U, f}^{\bullet}$. The diagram (2.27) exists by (2.20); its commutativity can be checked on the level of the underlying perverse sheaves which is (2.9), in light of Propositions A.1 A.2 in the Appendix. Note that (2.26) also includes the analogue of (2.10) in Theorem 2.13 according to which we have a matching of the monodromy actions $\tau_{U \times V, f \boxplus g} \cong \tau_{U, f} \stackrel{L}{\otimes} \tau_{V, g}$, as (2.26) holds in $\operatorname{MHM}\left(X \times Y ; T_{s}, N\right)$ rather than just $\operatorname{MHM}(X \times Y)$.

In this paper we will only ever apply Theorem 2.24 when $V=\mathbb{C}^{n}, g=$ $z_{1}^{2}+\cdots+z_{n}^{2}$ and $Y=\{0\}$. Combining (2.23) and (2.24) shows that

$$
\mathcal{H} \mathcal{V}_{\mathbb{C}, z^{2}}^{\bullet}=\left(\mathbb{Q}\left(-\frac{1}{2}\right)\right)\left(\frac{1}{2}\right) \cong \mathbb{Q}(0) \cong \mathbb{Q}_{\{0\}}^{H}
$$

Thus, by Theorem 2.24, $\mathbb{Q}_{\{0\}}^{H} \stackrel{T}{\bigotimes}^{T} \mathbb{Q}_{\{0\}}^{H} \cong \mathbb{Q}_{\{0\}}^{H}$, and induction on $n$, we see that

$$
\mathcal{H} \mathcal{V}_{\mathbb{C}^{n}, z_{1}^{2}+\cdots+z_{n}^{2}}^{\bullet} \mathbb{Q}_{\{0\}}^{H} \text {. }
$$

As for (2.12), this isomorphism is natural up to sign, depending on a choice of orientation for the complex Euclidean space $\left(\mathbb{C}^{n}, \mathrm{~d} z_{1}^{2}+\cdots+\mathrm{d} z_{n}^{2}\right)$. 


\section{Action of symmetries on vanishing cycles}

Here is our first main result.

Theorem 3.1. Let $U, V$ be smooth $\mathbb{C}$-schemes, $\Phi, \Psi: U \rightarrow V$ étale morphisms, and $f: U \rightarrow \mathbb{C}, g: V \rightarrow \mathbb{C}$ regular functions with $g \circ \Phi=f=g \circ \Psi$. Write $X=$ $\operatorname{Crit}(f)$ and $Y=\operatorname{Crit}(g)$ as $\mathbb{C}$-subschemes of $U, V$, so that $\left.\Phi\right|_{X},\left.\Psi\right|_{X}: X \rightarrow Y$ are étale morphisms. Suppose $\left.\Phi\right|_{X}=\left.\Psi\right|_{X}$. Then:

(a) As $\Phi, \Psi$ are étale, $\mathrm{d} \Phi: T U \rightarrow \Phi^{*}(T V), \mathrm{d} \Psi: T U \rightarrow \Psi^{*}(T V)$ are isomorphisms of vector bundles. Restricting to the reduced $\mathbb{C}$-subscheme $X^{\mathrm{red}}$ of $X$, and using $\left.\Phi\right|_{X^{\text {red }}}=\left.\Psi\right|_{X^{\text {red }}}$ as $\left.\Phi\right|_{X}=\left.\Psi\right|_{X}$, gives isomorphisms

$$
\begin{gathered}
\left.\mathrm{d} \Phi\right|_{X^{\text {red }}},\left.\mathrm{d} \Psi\right|_{X^{\text {red }}}:\left.\left.T U\right|_{X^{\text {red }}} \longrightarrow \Phi\right|_{X^{\text {red }}} ^{*}(T V), \\
\text { and thus }\left.\left.\mathrm{d} \Psi\right|_{X^{\text {red }}} ^{-1} \circ \mathrm{d} \Phi\right|_{X^{\text {red }}}:\left.\left.T U\right|_{X^{\text {red }}} \longrightarrow T U\right|_{X^{\text {red }}} .
\end{gathered}
$$

Hence $\operatorname{det}\left(\left.\left.\mathrm{d} \Psi\right|_{X^{\text {red }}} ^{-1} \circ \mathrm{d} \Phi\right|_{X^{\text {red }}}\right): X^{\text {red }} \rightarrow \mathbb{C} \backslash\{0\}$ is a regular function. Then $\operatorname{det}\left(\left.\left.\mathrm{d} \Psi\right|_{X^{\text {red }}} ^{-1} \circ \mathrm{d} \Phi\right|_{X^{\text {red }}}\right)$ is a locally constant map $X^{\text {red }} \rightarrow\{ \pm 1\} \subset \mathbb{C} \backslash\{0\}$.

(b) Definition 2.15 defines isomorphisms $\mathcal{P} \mathcal{V}_{\Phi}, \mathcal{P} \mathcal{V}_{\Psi}:\left.\mathcal{P} \mathcal{V}_{U, f}^{\bullet} \rightarrow \Phi\right|_{X} ^{*}\left(\mathcal{P} \mathcal{V}_{V, g}^{\bullet}\right)$ in $\operatorname{Perv}(X)$. These are related by

$$
\mathcal{P} \mathcal{V}_{\Phi}=\operatorname{det}\left(\left.\left.\mathrm{d} \Psi\right|_{X^{\mathrm{red}}} ^{-1} \circ \mathrm{d} \Phi\right|_{X^{\mathrm{red}}}\right) \cdot \mathcal{P} \mathcal{V}_{\Psi}
$$

regarding $\operatorname{det}\left(\left.\left.\mathrm{d} \Psi\right|_{X^{\text {red }}} ^{-1} \circ \mathrm{d} \Phi\right|_{X^{\text {red }}}\right): X \rightarrow\{ \pm 1\}$ as a locally constant map of topological spaces, where $X, X^{\text {red }}$ have the same topological space.

The analogues of these results also hold for $\mathscr{D}$-modules and mixed Hodge modules on $\mathbb{C}$-schemes, and (with $\Phi, \Psi$ local biholomorphisms and $f, g$ analytic functions) for perverse sheaves, $\mathscr{D}$-modules and mixed Hodge modules on complex analytic spaces, as in $\$ 2.6-12.10$.

By taking $U=V, f=g, \Phi$ an isomorphism and $\Psi=\mathrm{id}_{U}$, we deduce a result on the action of symmetries on perverse sheaves of vanishing cycles:

Corollary 3.2. Let $U$ be a smooth $\mathbb{C}$-scheme, $\Phi: U \rightarrow U$ an isomorphism, and $f: U \rightarrow \mathbb{C}$ be regular with $f \circ \Phi=f$. Write $X=\operatorname{Crit}(f)$ as a $\mathbb{C}$-subscheme of $U$ and $X^{\text {red }}$ for its reduced $\mathbb{C}$-subscheme, and suppose $\left.\Phi\right|_{X}=\operatorname{id}_{X}$. Then $\operatorname{det}\left(\left.\mathrm{d} \Phi\right|_{X^{\mathrm{red}}}:\left.\left.T U\right|_{X^{\mathrm{red}}} \rightarrow T U\right|_{X^{\mathrm{red}}}\right)$ is a locally constant map $X^{\mathrm{red}} \rightarrow\{ \pm 1\}$, and $\mathcal{P} \mathcal{V}_{\Phi}: \mathcal{P} \mathcal{V}_{U, f}^{\bullet} \cong \mathcal{P} \mathcal{V}_{U, f}^{\bullet}$ in $\operatorname{Perv}(X)$ from Definition 2.15 is multiplication by $\operatorname{det}\left(\left.\mathrm{d} \Phi\right|_{X^{\text {red }}}\right)= \pm 1$. The analogues hold in the settings of $\$ 2.6$ - 92.10 .

Example 3.3. Let $U=V=\mathbb{C}^{n}$ and $f\left(z_{1}, \ldots, z_{n}\right)=g\left(z_{1}, \ldots, z_{n}\right)=z_{1}^{2}+$ $\cdots+z_{n}^{2}$, so that $X=Y=\{0\} \subset \mathbb{C}^{n}$. Let $\Phi, \Psi \in \mathrm{O}(n, \mathbb{C})$ be orthogonal matrices, so that $\operatorname{det} \Phi, \operatorname{det} \Psi \in\{ \pm 1\}$ and $\Phi, \Psi: \mathbb{C}^{n} \rightarrow \mathbb{C}^{n}$ are isomorphisms with $f=g \circ \Phi=g \circ \Phi$ and $\left.\Phi\right|_{\{0\}}=\left.\Psi\right|_{\{0\}}=\operatorname{id}_{\{0\}}$. In Theorem 3.1(a) we have

$$
\left.\left.\mathrm{d} \Psi\right|_{X^{\text {red }}} ^{-1} \circ \mathrm{d} \Phi\right|_{X^{\text {red }}}=\Psi^{-1} \circ \Phi: \mathbb{C}^{n} \longrightarrow \mathbb{C}^{n},
$$


so that $\operatorname{det}\left(\left.\left.\mathrm{d} \Psi\right|_{X^{\text {red }}} ^{-1} \circ \mathrm{d} \Phi\right|_{X^{\text {red }}}\right)=\operatorname{det} \Psi^{-1} \operatorname{det} \Phi= \pm 1$.

For Theorem[3.1(b), Example 2.16] shows that $\mathcal{P} \mathcal{V}_{\Phi}, \mathcal{P} \mathcal{V}_{\Psi}: A_{\{0\}} \rightarrow A_{\{0\}}$ are multiplication by $\operatorname{det} \Phi, \operatorname{det} \Psi$, so $\mathcal{P} \mathcal{V}_{\Phi}=\left(\operatorname{det} \Psi^{-1} \operatorname{det} \Phi\right) \cdot \mathcal{P} \mathcal{V}_{\Psi}$, as in (3.1).

The proof of Theorem 3.1(b) uses the following proposition. To interpret it, pretend for simplicity that the étale morphisms $\left.\pi_{U}\right|_{W_{t}}: W_{t} \rightarrow U$ in (b) are invertible. Then $\Theta_{t}:=\left.\left.\pi_{V}\right|_{W_{t}} \circ \pi_{U}\right|_{W_{t}} ^{-1}$ for $t \in \mathbb{C}$ are a 1-parameter family of morphisms $U \rightarrow V$, which satisfy $f=g \circ \Theta_{t}$ and $\left.\Theta_{t}\right|_{X}=\left.\Phi\right|_{X}=\left.\Psi\right|_{X}$ for $t \in \mathbb{C}$, with $\Theta_{0}=\Phi$ and $\Theta_{1}=\Psi$. Thus, modulo taking étale covers of $U$, the family $\left\{\Theta_{t}: t \in \mathbb{C}\right\}$ interpolates between $\Phi$ and $\Psi$.

Proposition 3.4. Let $U, V$ be smooth $\mathbb{C}$-schemes, $\Phi, \Psi: U \rightarrow V$ étale morphisms, and $f: U \rightarrow \mathbb{C}, g: V \rightarrow \mathbb{C}$ regular functions with $g \circ \Phi=f=$ $g \circ \Psi$. Write $X=\operatorname{Crit}(f)$ and $Y=\operatorname{Crit}(g)$ as $\mathbb{C}$-subschemes of $U, V$, so that $\left.\Phi\right|_{X},\left.\Psi\right|_{X}: X \rightarrow Y$ are étale. Suppose $\left.\Phi\right|_{X}=\left.\Psi\right|_{X}$, and $x \in X$ such that $\left.\left.\mathrm{d} \Psi\right|_{x} ^{-1} \circ \mathrm{d} \Phi\right|_{x}: T_{x} U \rightarrow T_{x} U$ satisfies $\left(\left.\left.\mathrm{d} \Psi\right|_{x} ^{-1} \circ \mathrm{d} \Phi\right|_{x}-\mathrm{id}_{T_{x} U}\right)^{2}=0$. Then there exist a smooth $\mathbb{C}$-scheme $W$ and morphisms $\pi_{\mathbb{C}}: W \rightarrow \mathbb{C}, \pi_{U}: W \rightarrow U$, $\pi_{V}: W \rightarrow V$ and $\iota: \mathbb{C} \rightarrow W$ such that:

(a) $\pi_{\mathbb{C}} \circ \iota(t)=t, \pi_{U} \circ \iota(t)=x$ and $\pi_{V} \circ \iota(t)=\Phi(x)$ for all $t \in \mathbb{C}$;

(b) $\pi_{\mathbb{C}} \times \pi_{U}: W \rightarrow \mathbb{C} \times U$ and $\pi_{\mathbb{C}} \times \pi_{V}: W \rightarrow \mathbb{C} \times V$ are étale. Thus, $W_{t}:=\pi_{\mathbb{C}}^{-1}(t)$ is a smooth $\mathbb{C}$-scheme for each $t \in \mathbb{C}$, and $\left.\pi_{U}\right|_{W_{t}}: W_{t} \rightarrow U,\left.\pi_{V}\right|_{W_{t}}: W_{t} \rightarrow$ $V$ are étale, and $\iota(t) \in W_{t}$ with $\pi_{U}: \iota(t) \mapsto x, \pi_{V}: \iota(t) \mapsto \Phi(x)$;

(c) $h:=f \circ \pi_{U}=g \circ \pi_{V}: W \rightarrow \mathbb{C}$. Thus, $\left.\left(\pi_{\mathbb{C}} \times \pi_{U}\right)\right|_{Z}: Z \rightarrow \mathbb{C} \times X$ and $\left.\left(\pi_{\mathbb{C}} \times \pi_{V}\right)\right|_{Z}: Z \rightarrow \mathbb{C} \times Y$ are étale, where $Z:=\operatorname{Crit}(h)$

(d) $\left.\left.\Phi\right|_{X} \circ \pi_{U}\right|_{Z}=\left.\left.\Psi\right|_{X} \circ \pi_{U}\right|_{Z}=\left.\pi_{V}\right|_{Z}: Z \rightarrow Y \subseteq V$; and

(e) $\left.\Phi \circ \pi_{U}\right|_{W_{0}}=\left.\pi_{V}\right|_{W_{0}}$ and $\left.\Psi \circ \pi_{U}\right|_{W_{1}}=\left.\pi_{V}\right|_{W_{1}}$, for $W_{0}, W_{1}$ as in (b).

We will prove Proposition 3.4 in 3.1 and Theorem 3.1 in 3.23 .4

\subsection{Proof of Proposition 3.4}

Let $U, V, \Phi, \Psi, f, g, X, Y, x$ be as in Proposition 3.4. Choose a Zariski open neighbourhood $V^{\prime}$ of $\Phi(x)=\Psi(x)$ in $V$ and étale coordinates $\left(z_{1}, \ldots, z_{n}\right): V^{\prime} \rightarrow \mathbb{C}^{n}$ on $V^{\prime}$, with $z_{1}=\cdots=z_{n}=0$ at $\Phi(x)$. Let $m$ be the rank of the symmetric matrix $\left(\left.\frac{\partial^{2} g}{\partial z_{i} \partial z_{j}}\right|_{\Phi(x)}\right)_{i, j=1}^{n}$, so that $m \in\{0, \ldots, n\}$. By applying an element of $\mathrm{GL}(n, \mathbb{C})$ to the coordinates $\left(z_{1}, \ldots, z_{n}\right)$ we can suppose that

$$
\left.\frac{\partial^{2} g}{\partial z_{i} \partial z_{j}}\right|_{\Phi(x)}= \begin{cases}1, & i=j \in\{1, \ldots, m\} \\ 0, & \text { otherwise. }\end{cases}
$$

Then $\frac{1}{2} \frac{\partial g}{\partial z_{i}}$ agrees with $z_{i}$ to first order at $\Phi(x)$ for $i=1, \ldots, m$, so replacing $z_{i}$ by $\frac{1}{2} \frac{\partial g}{\partial z_{i}}$ for $i=1, \ldots, m$ and making $V^{\prime}$ smaller, we can suppose (3.2) holds and $z_{1}, \ldots, z_{m}$ lie in the ideal $\left(\frac{\partial g}{\partial z_{i}}, i=1, \ldots, n\right)$ in $\mathcal{O}_{V^{\prime}}$. Thus we may write

$$
z_{i}=\sum_{j=1}^{n} A_{i j} \cdot \frac{\partial g}{\partial z_{j}}, \quad i=1, \ldots, m
$$


where $A_{i j}: V^{\prime} \rightarrow \mathbb{A}^{1}$ are regular functions for $i=1, \ldots, m$ and $j=1, \ldots, n$. Taking $\frac{\partial}{\partial z_{j}}$ of (3.3) for $j=1, \ldots, m$ and using (3.2) gives

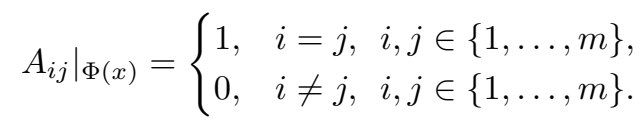

Set $U^{\prime}=\Phi^{-1}\left(V^{\prime}\right) \cap \Psi^{-1}\left(V^{\prime}\right)$, so that $U^{\prime}$ is a Zariski open neighbourhood of $x$ in $U$. Define étale coordinates $\left(x_{1}, \ldots, x_{n}\right): U^{\prime} \rightarrow \mathbb{C}^{n}$ and $\left(y_{1}, \ldots, y_{n}\right): U^{\prime} \rightarrow$ $\mathbb{C}^{n}$ by $x_{i}=z_{i} \circ \Phi$ and $y_{i}=z_{i} \circ \Psi$, so that $x_{1}=\cdots=x_{n}=y_{1}=\cdots=y_{n}=0$ at $x$. Since $f=g \circ \Phi=g \circ \Psi$ we have $\frac{\partial f}{\partial x_{j}}=\frac{\partial g}{\partial z_{j}} \circ \Phi$ and $\frac{\partial f}{\partial y_{j}}=\frac{\partial g}{\partial z_{j}} \circ \Psi$. Thus (3.2) and (3.3) imply that

$$
\begin{gathered}
\left.\frac{\partial^{2} f}{\partial x_{i} \partial x_{j}}\right|_{x}=\left.\frac{\partial^{2} f}{\partial y_{i} \partial y_{j}}\right|_{x}= \begin{cases}1, & i=j \in\{1, \ldots, m\}, \\
0, & \text { otherwise },\end{cases} \\
x_{i}=\sum_{j=1}^{n}\left(A_{i j} \circ \Phi\right) \cdot \frac{\partial f}{\partial x_{j}}, \quad y_{i}=\sum_{j=1}^{n}\left(A_{i j} \circ \Psi\right) \cdot \frac{\partial f}{\partial y_{j}}, \quad i=1, \ldots, m .
\end{gathered}
$$

Now $\left.\mathrm{d} \Phi\right|_{x}: T_{x} U \rightarrow T_{\Phi(x)} V$ maps $\frac{\partial}{\partial x_{j}} \mapsto \frac{\partial}{\partial z_{j}}$, as $x_{j}=z_{j} \circ \Phi$, and $\left.\mathrm{d} \Psi\right|_{x}:$ $T_{x} U \rightarrow T_{\Phi(x)} V$ maps $\frac{\partial}{\partial y_{j}} \mapsto \frac{\partial}{\partial z_{j}}$. Hence $\left.\left.\mathrm{d} \Psi\right|_{x} ^{-1} \circ \mathrm{d} \Phi\right|_{x}: T_{x} U \rightarrow T_{x} U$ maps $\frac{\partial}{\partial x_{j}} \mapsto \frac{\partial}{\partial y_{j}}=\sum_{i=1}^{n} \frac{\partial x_{i}}{\partial y_{j}} \cdot \frac{\partial}{\partial x_{i}}$. Define $B_{i j} \in \mathbb{C}$ for $i, j=1, \ldots, n$ by

$$
\delta_{i j}+B_{i j}=\left.\frac{\partial x_{i}}{\partial y_{j}}\right|_{x} .
$$

Then $\left(\delta_{i j}+B_{i j}\right)_{i, j=1}^{n}$ is the matrix of $\left.\left.\mathrm{d} \Psi\right|_{x} ^{-1} \circ \mathrm{d} \Phi\right|_{x}$ w.r.t. the basis $\frac{\partial}{\partial x_{1}}, \ldots, \frac{\partial}{\partial x_{n}}$, and $\left(B_{i j}\right)_{i, j=1}^{n}$ is the matrix of $\left.\left.\mathrm{d} \Psi\right|_{x} ^{-1} \circ \mathrm{d} \Phi\right|_{x}-\mathrm{id}_{T_{x} U}$, so by assumption $\left(B_{i j}\right)^{2}=$ 0 . Therefore the inverse matrix of $\left(\delta_{i j}+B_{i j}\right)$ is $\left(\delta_{i j}-B_{i j}\right)$, so (3.7) gives

$$
\delta_{i j}-B_{i j}=\left.\frac{\partial y_{i}}{\partial x_{j}}\right|_{x} .
$$

More generally, $\left(\delta_{i j}+t B_{i j}\right)$ is invertible for $t \in \mathbb{C}$, with inverse $\left(\delta_{i j}-t B_{i j}\right)$.

Now $\left.\Phi\right|_{X}=\left.\Psi\right|_{X}$ implies that $\left.\left.\mathrm{d} \Psi\right|_{x} ^{-1} \circ \mathrm{d} \Phi\right|_{x}$ is the identity on $T_{x} X \subseteq T_{x} U$, and $T_{x} X=\operatorname{Ker}\left(\operatorname{Hess}_{x} f\right)=\left\langle\frac{\partial}{\partial x_{m+1}}, \ldots, \frac{\partial}{\partial x_{n}}\right\rangle$ by (3.5), so

$$
B_{i j}=0 \quad \text { for all } i=1, \ldots, n \text { and } j=m+1, \ldots, n \text {. }
$$

We have $\left.\frac{\partial^{2} f}{\partial y_{i} \partial y_{l}}\right|_{x}=\left.\sum_{j, k} \frac{\partial x_{j}}{\partial y_{i}} \frac{\partial^{2} f}{\partial x_{j} \partial x_{k}} \frac{\partial x_{k}}{\partial y_{l}}\right|_{x},\left.\frac{\partial^{2} f}{\partial x_{i} \partial x_{l}}\right|_{x}=\left.\sum_{j, k} \frac{\partial y_{j}}{\partial x_{i}} \frac{\partial^{2} f}{\partial y_{j} \partial y_{k}} \frac{\partial y_{k}}{\partial x_{l}}\right|_{x}$, which by (3.5) and (3.7)-(3.9) give equations equivalent to

$$
B_{i j}+B_{j i}=\sum_{k=1}^{n} B_{k i} B_{k j}=0 \quad \text { for all } i, j=1, \ldots, m .
$$

Define regular $t^{\prime}, x_{1}^{\prime}, \ldots, x_{n}^{\prime}, y_{1}^{\prime}, \ldots, y_{n}^{\prime}, z_{1}^{\prime}, \ldots, z_{n}^{\prime}: \mathbb{C} \times U^{\prime} \times V^{\prime} \rightarrow \mathbb{C}$ by

$$
\begin{aligned}
t^{\prime}(t, u, v) & =t, & x_{i}^{\prime}(t, u, v) & =x_{i}(u)=z_{i} \circ \Phi(u), \\
y_{i}^{\prime}(t, u, v) & =y_{i}(u)=z_{i} \circ \Psi(u), & z_{i}^{\prime}(t, u, v) & =z_{i}(v) .
\end{aligned}
$$


Then $\left(t^{\prime}, y_{1}^{\prime}, \ldots, y_{n}^{\prime}, z_{1}^{\prime}, \ldots, z_{n}^{\prime}\right)$ are étale coordinates on $\mathbb{C} \times U^{\prime} \times V^{\prime}$.

Let $S$ be an affine Zariski open neighbourhood of $\mathbb{C} \times(x, \Phi(x))$ in $\mathbb{C} \times U^{\prime} \times V^{\prime}$, satisfying a series of smallness conditions we will give during the proof. Regard $\left(t^{\prime}, y_{1}^{\prime}, \ldots, y_{n}^{\prime}, z_{1}^{\prime}, \ldots, z_{n}^{\prime}\right)$ as étale coordinates on $S$, and write $\pi_{\mathbb{C}}: S \rightarrow \mathbb{C}$, $\pi_{U}: S \rightarrow U, \pi_{V}: S \rightarrow V$ for the projections. We will work with (sheaves of) ideals in $\mathcal{O}_{S}$, using notation $\left(x_{i}^{\prime}-z_{i}^{\prime}, i=1, \ldots, n\right)$ to denote the ideal generated by the functions $x_{1}^{\prime}-z_{1}^{\prime}, \ldots, x_{n}^{\prime}-z_{n}^{\prime}$, and $f \circ \pi_{U}-g \circ \pi_{V} \in\left(x_{i}^{\prime}-z_{i}^{\prime}, i=1, \ldots, n\right)$ to mean that $f \circ \pi_{U}-g \circ \pi_{V} \in H^{0}\left(\mathcal{O}_{S}\right)$ is a section of the ideal $\left(x_{i}^{\prime}-z_{i}^{\prime}, i=1, \ldots, n\right)$. Write $I_{X} \subset \mathcal{O}_{U}, I_{Y} \subset \mathcal{O}_{V}$ for the ideals of functions on $U, V$ vanishing on $X, Y$, and $\pi_{U}^{-1}\left(I_{X}\right), \pi_{V}^{-1}\left(I_{Y}\right) \subset \mathcal{O}_{S}$ for the preimage ideals.

Since $x_{i}=z_{i} \circ \Phi$, the functions $x_{i}^{\prime}-z_{i}^{\prime}$ for $i=1, \ldots, n$ vanish on the smooth, closed $\mathbb{C}$-subscheme $(\mathbb{C} \times($ id $\times \Phi)(U)) \cap S$ in $S$, and locally these functions cut out this $\mathbb{C}$-subscheme. So making $S$ smaller we can suppose $(\mathbb{C} \times(\mathrm{id} \times \Phi)(U)) \cap S$ is the $\mathbb{C}$-subscheme $x_{1}^{\prime}-z_{1}^{\prime}=\cdots=x_{n}^{\prime}-z_{n}^{\prime}=0$ in $S$. As $f=g \circ \Phi$, the function $f \circ \pi_{U}-g \circ \pi_{V}$ is zero on $(\mathbb{C} \times(\operatorname{id} \times \Phi)(U)) \cap S$. Hence

$$
f \circ \pi_{U}-g \circ \pi_{V} \in\left(x_{i}^{\prime}-z_{i}^{\prime}, i=1, \ldots, n\right) \subset \mathcal{O}_{S} .
$$

Lifting (3.11) from $\left(x_{i}^{\prime}-z_{i}^{\prime}, i=1, \ldots, n\right)$ to $\left(x_{i}^{\prime}-z_{i}^{\prime}, i=1, \ldots, n\right)^{2}$, making $S$ smaller if necessary, we may choose regular $C_{i}: S \rightarrow \mathbb{C}$ for $i=1, \ldots, n$ with

$$
f \circ \pi_{U}-g \circ \pi_{V}-\sum_{i=1}^{n} C_{i} \cdot\left(x_{i}^{\prime}-z_{i}^{\prime}\right) \in\left(x_{i}^{\prime}-z_{i}^{\prime}, i=1, \ldots, n\right)^{2} .
$$

Apply $\frac{\partial}{\partial z_{i}^{\prime}}$ to (3.12), using the étale coordinates $\left(t^{\prime}, y_{1}^{\prime}, \ldots, y_{n}^{\prime}, z_{1}^{\prime}, \ldots, z_{n}^{\prime}\right)$ on $S$. Since $\frac{\partial}{\partial z_{i}^{\prime}}\left(g \circ \pi_{V}\right)=\frac{\partial g}{\partial z_{i}} \circ \pi_{V}$ and $\frac{\partial}{\partial z_{i}^{\prime}}\left(f \circ \pi_{U}\right)=0=\frac{\partial x_{j}^{\prime}}{\partial z_{i}^{\prime}}$, this gives

$$
C_{i}-\frac{\partial g}{\partial z_{i}} \circ \pi_{V} \in\left(x_{i}^{\prime}-z_{i}^{\prime}, i=1, \ldots, n\right) .
$$

Combining this with (3.12) yields

$$
f \circ \pi_{U}-g \circ \pi_{V}-\sum_{i=1}^{n}\left(\frac{\partial g}{\partial z_{i}} \circ \pi_{V}\right) \cdot\left(x_{i}^{\prime}-z_{i}^{\prime}\right) \in\left(x_{i}^{\prime}-z_{i}^{\prime}, i=1, \ldots, n\right)^{2} .
$$

So making $S$ smaller we can choose regular $D_{i j}: S \rightarrow \mathbb{C}$ for $i, j=1, \ldots, n$ with $D_{i j}=D_{j i}$ and

$$
f \circ \pi_{U}-g \circ \pi_{V}=\sum_{i=1}^{n}\left(\frac{\partial g}{\partial z_{i}} \circ \pi_{V}\right) \cdot\left(x_{i}^{\prime}-z_{i}^{\prime}\right)+\sum_{i, j=1}^{n} D_{i j} \cdot\left(x_{i}^{\prime}-z_{i}^{\prime}\right)\left(x_{j}^{\prime}-z_{j}^{\prime}\right) .
$$

Similarly, starting from $y_{i}=z_{i} \circ \Psi$ and $f=g \circ \Psi$ we may choose regular $E_{i j}: S \rightarrow \mathbb{C}$ for $i, j=1, \ldots, n$ with $E_{i j}=E_{j i}$ and

$$
f \circ \pi_{U}-g \circ \pi_{V}=\sum_{i=1}^{n}\left(\frac{\partial g}{\partial z_{i}} \circ \pi_{V}\right) \cdot\left(y_{i}^{\prime}-z_{i}^{\prime}\right)+\sum_{i, j=1}^{n} E_{i j} \cdot\left(y_{i}^{\prime}-z_{i}^{\prime}\right)\left(y_{j}^{\prime}-z_{j}^{\prime}\right) .
$$


Applying $\frac{\partial^{2}}{\partial z_{i}^{\prime} \partial z_{j}^{\prime}}$ to (3.13) and (3.14), restricting to $(t, x, \Phi(x))$ for $t \in \mathbb{C}$, noting that $x_{i}^{\prime}=y_{i}^{\prime}=z_{i}^{\prime}=0$ at $(t, x, \Phi(x))$, and using (3.2), we deduce that

$$
\begin{aligned}
D_{i j}(t, x, \Phi(x))=E_{i j}(t, x, \Phi(x)) & =\left.\frac{1}{2} \frac{\partial^{2} g}{\partial z_{i} \partial z_{j}}\right|_{\Phi(x)} \\
& = \begin{cases}\frac{1}{2}, & i=j \in\{1, \ldots, m\}, \\
0, & \text { otherwise. }\end{cases}
\end{aligned}
$$

Summing $1-t^{\prime}$ times (3.13) with $t^{\prime}$ times (3.14) and rearranging yields

$$
\begin{aligned}
& f \circ \pi_{U}-g \circ \pi_{V}=\sum_{i=1}^{n}\left[\frac{\partial g}{\partial z_{i}} \circ \pi_{V}+2 t^{\prime}\left(1-t^{\prime}\right) \sum_{j=1}^{n}\left(D_{i j}-E_{i j}\right)\left(x_{j}^{\prime}-y_{j}^{\prime}\right)\right] \\
& \cdot\left(\left(1-t^{\prime}\right) x_{i}^{\prime}+t^{\prime} y_{i}^{\prime}-z_{i}^{\prime}\right) \\
&+\sum_{i, j=1}^{n}\left[\left(1-t^{\prime}\right) D_{i j}+t^{\prime} E_{i j}\right] \cdot\left(\left(1-t^{\prime}\right) x_{i}^{\prime}+t^{\prime} y_{i}^{\prime}-z_{i}^{\prime}\right)\left(\left(1-t^{\prime}\right) x_{j}^{\prime}+t^{\prime} y_{j}^{\prime}-z_{j}^{\prime}\right) \\
&+\sum_{i, j=1}^{n} t^{\prime}\left(1-t^{\prime}\right)\left[t^{\prime} D_{i j}+\left(1-t^{\prime}\right) E_{i j}\right] \cdot\left(x_{i}^{\prime}-y_{i}^{\prime}\right)\left(x_{j}^{\prime}-y_{j}^{\prime}\right) .
\end{aligned}
$$

Since $x_{i}^{\prime}-y_{i}^{\prime}=\left(x_{i}-y_{i}\right) \circ \pi_{U}$, and $\left.\left(x_{i}-y_{i}\right)\right|_{X}=\left.z_{i} \circ \Phi\right|_{X}-\left.z_{i} \circ \Psi\right|_{X}=0$ as $\left.\Phi\right|_{X}=\left.\Psi\right|_{X}$, we see that $x_{i}^{\prime}-y_{i}^{\prime} \in \pi_{U}^{-1}\left(I_{X}\right)$. Thus making $S$ smaller if necessary, we may choose regular $F_{i j}: S \rightarrow \mathbb{C}$ such that

$$
x_{i}^{\prime}-y_{i}^{\prime}=\sum_{j=1}^{n} F_{i j} \cdot\left(\frac{\partial f}{\partial y_{j}} \circ \pi_{U}\right) \text { for } i=1, \ldots, n .
$$

Furthermore, by (3.6) when $i=1, \ldots, m$ we may take

$$
F_{i j}=\left(\sum_{k=1}^{n}\left(A_{i k} \circ \Phi\right) \cdot \frac{\partial y_{j}}{\partial x_{k}}-A_{i j} \circ \Psi\right) \circ \pi_{U} .
$$

Restricting to $(t, x, \Phi(x))$ and using (3.4), (3.8), (3.9) and $\Phi(x)=\Psi(x)$ gives

$$
F_{i j}(t, x, \Phi(x))=-B_{j i} \quad \text { for } i=1, \ldots, m \text { and } j=1, \ldots, n .
$$

Applying $\frac{\partial}{\partial x_{i}^{\prime}}$ to equation (3.13) shows that

$$
\frac{\partial g}{\partial z_{i}} \circ \pi_{V}-\frac{\partial f}{\partial x_{i}} \circ \pi_{U} \in\left(x_{j}^{\prime}-z_{j}^{\prime}, j=1, \ldots, n\right) .
$$

Thus we may write

$$
\frac{\partial g}{\partial z_{i}} \circ \pi_{V}=\sum_{j=1}^{n}\left(\frac{\partial y_{j}}{\partial x_{i}} \circ \pi_{U}\right) \cdot\left(\frac{\partial f}{\partial y_{j}} \circ \pi_{U}\right)+\sum_{j=1}^{n} G_{i j} \cdot\left(x_{j}^{\prime}-z_{j}^{\prime}\right),
$$

where $G_{i j}: S \rightarrow \mathbb{C}$ are regular. Applying $\frac{\partial}{\partial z_{j}^{\prime}}$ to (3.19), restricting to $(t, x, \Phi(x))$ and using (3.2) yields

$$
G_{i j}(t, x, \Phi(x))= \begin{cases}-1, & i=j \in\{1, \ldots, m\} \\ 0, & \text { otherwise }\end{cases}
$$


From (3.17) and (3.19) we see that

$$
\frac{\partial g}{\partial z_{i}} \circ \pi_{V}=\sum_{j=1}^{n} H_{i j} \cdot\left(\frac{\partial f}{\partial y_{j}} \circ \pi_{U}\right)+\sum_{j=1}^{n} G_{i j} \cdot\left(\left(1-t^{\prime}\right) x_{j}^{\prime}+t^{\prime} y_{j}^{\prime}-z_{j}^{\prime}\right),
$$

where $H_{i j}=\frac{\partial y_{j}}{\partial x_{i}} \circ \pi_{U}+t^{\prime} \sum_{k=1}^{n} G_{i k} F_{k j}$, so that from equations (3.8), (3.9), (3.18) and (3.20) we deduce that

$$
H_{i j}(t, x, \Phi(x))=\delta_{i j}-(1-t) B_{j i} .
$$

Combining (3.16), (3.17) and (3.21) gives

$$
\begin{aligned}
& f \circ \pi_{U}-g \circ \pi_{V}-\sum_{i=1}^{n} I_{i} \cdot\left(\left(1-t^{\prime}\right) x_{i}^{\prime}+t^{\prime} y_{i}^{\prime}-z_{i}^{\prime}\right) \\
& -\sum_{i, j, k, l=1}^{n} t^{\prime}\left(1-t^{\prime}\right)\left[t^{\prime} D_{i j}+\left(1-t^{\prime}\right) E_{i j}\right] F_{i k} F_{j l} \cdot\left(\frac{\partial f}{\partial y_{k}} \circ \pi_{U}\right)\left(\frac{\partial f}{\partial y_{l}} \circ \pi_{U}\right) \\
& \quad \in\left(\left(1-t^{\prime}\right) x_{i}^{\prime}+t^{\prime} y_{i}^{\prime}-z_{i}^{\prime}, i=1, \ldots, n\right)^{2}, \\
& \text { where } \quad I_{i}=\sum_{j=1}^{n}\left[H_{i j}+2 t^{\prime}\left(1-t^{\prime}\right) \sum_{k=1}^{n}\left(D_{i k}-E_{i k}\right) F_{k j}\right] \cdot\left(\frac{\partial f}{\partial y_{j}} \circ \pi_{U}\right) .
\end{aligned}
$$

Consider the matrix of functions $\left[H_{i j}+\cdots\right]_{i, j=1}^{n}$ appearing in (3.24). Equations (3.15) and (3.22) imply that at $(t, x, \Phi(x))$ this reduces to $\left(\delta_{i j}-(1-t) B_{j i}\right)$, which is invertible from above. Thus, making $S$ smaller, we can suppose that $\left[H_{i j}+\cdots\right]_{i, j=1}^{n}$ in (3.24) is an invertible matrix on $S$. Write $\left[J_{i j}\right]_{i, j=1}^{n}$ for the inverse matrix. Then we have

$$
\begin{aligned}
& \sum_{i, j, k, l=1}^{n} t^{\prime}\left(1-t^{\prime}\right)\left[t^{\prime} D_{i j}+\left(1-t^{\prime}\right) E_{i j}\right] F_{i k} F_{j l} \cdot\left(\frac{\partial f}{\partial y_{k}} \circ \pi_{U}\right)\left(\frac{\partial f}{\partial y_{l}} \circ \pi_{U}\right) \\
& \quad=t^{\prime}\left(1-t^{\prime}\right) \sum_{i, j=1}^{n} K_{i j} \cdot I_{i} I_{j}, \quad \text { where } \\
& K_{i j}=\sum_{k, l, p, q=1}^{n} t^{\prime}\left(1-t^{\prime}\right)\left[t^{\prime} D_{k l}+\left(1-t^{\prime}\right) E_{k l}\right] F_{k p} F_{l q} J_{p i} J_{q j} .
\end{aligned}
$$

Using (3.9), (3.10), (3.15) and (3.18) we find that

$$
K_{i j}(t, x, \Phi(x))=0 \quad \text { for all } t \in \mathbb{C} .
$$

Combining (3.23) and (3.25), making $S$ smaller if necessary we may write

$$
\begin{aligned}
f & \circ \pi_{U}-g \circ \pi_{V}=\sum_{i=1}^{n} I_{i} \cdot\left(\left(1-t^{\prime}\right) x_{i}^{\prime}+t^{\prime} y_{i}^{\prime}-z_{i}^{\prime}\right)+t^{\prime}\left(1-t^{\prime}\right) \sum_{i, j=1}^{n} K_{i j} \cdot I_{i} I_{j} \\
& +\sum_{i, j=1}^{n} L_{i j} \cdot\left(\left(1-t^{\prime}\right) x_{i}^{\prime}+t^{\prime} y_{i}^{\prime}-z_{i}^{\prime}\right)\left(\left(1-t^{\prime}\right) x_{j}^{\prime}+t^{\prime} y_{j}^{\prime}-z_{j}^{\prime}\right),
\end{aligned}
$$

for regular $L_{i j}: S \rightarrow \mathbb{C}$ for $i, j=1, \ldots, n$. 
Write $\left(r_{i j}\right)_{i, j=1}^{n}$ for the coordinates on $\mathbb{C}^{n^{2}}$. Let $T$ be a Zariski open neighbourhood of $\mathbb{C} \times\left(x, \Phi(x),(0)_{i, j=1}^{n}\right)$ in $S \times \mathbb{C}^{n^{2}}$ to be chosen shortly, and let $W$ be the closed $\mathbb{C}$-subscheme of $T$ defined by

$$
\begin{aligned}
W=\{ & \left(t, u, v,\left(r_{i j}\right)_{i, j=1}^{n}\right) \in T \subseteq S \times \mathbb{C}^{n^{2}} \subseteq \mathbb{C} \times U \times V \times \mathbb{C}^{n^{2}}: \\
& \left(\left(1-t^{\prime}\right) x_{i}^{\prime}+t^{\prime} y_{i}^{\prime}-z_{i}^{\prime}\right)(t, u, v)=\sum_{j=1}^{n} r_{i j} \cdot I_{j}(t, u, v), \quad i=1, \ldots, n, \\
& \left.r_{i j}+t(1-t) K_{i j}(t, u, v)+\sum_{k, l=1}^{n} L_{k l}(t, u, v) \cdot r_{k i} r_{l j}=0, i, j=1, \ldots, n\right\} .
\end{aligned}
$$

Define $\mathbb{C}$-scheme morphisms $\pi_{\mathbb{C}}: W \rightarrow \mathbb{C}, \pi_{U}: W \rightarrow U, \pi_{V}: W \rightarrow V$ to map $\left(t, u, v,\left(r_{i j}\right)_{i, j=1}^{n}\right)$ to $t, u, v$, respectively.

At $(t, x, \Phi(x)) \in S$ for $t \in \mathbb{C}$ we have $x_{i}^{\prime}=y_{i}^{\prime}=z_{i}^{\prime}=0$, and $I_{i}=0$ by (3.24) as $\left.\frac{\partial f}{\partial y_{j}}\right|_{x}=0$, and $K_{i j}=0$ by (3.26). Hence $\left(t, x, \Phi(x),(0)_{i, j=1}^{n}\right)$ satisfies the equations of (3.28), and lies in $W$. Define $\iota: \mathbb{C} \rightarrow W$ by

$$
\iota(t)=\left(t, x, \Phi(x),(0)_{i, j=1}^{n}\right) .
$$

Now $T \subseteq \mathbb{C} \times U \times V \times \mathbb{C}^{n^{2}}$ is smooth of dimension $1+n+n+n^{2}$, and in (3.28) we impose $n+n^{2}$ equations, so the expected dimension of $W$ is $(1+2 n+$ $\left.n^{2}\right)-\left(n+n^{2}\right)=n+1$. The linearizations of the $n+n^{2}$ equations in (3.28) at $\left(t, u, v,\left(r_{i j}\right)_{i, j=1}^{n}\right)=\left(t, x, \Phi(x),(0)_{i, j=1}^{n}\right)=\iota(t)$ are

$$
\begin{aligned}
\left.\mathrm{d} y_{i}\right|_{x}(\delta u)-\left.\mathrm{d} z_{i}\right|_{\Phi(x)}(\delta v)=0, & i=1, \ldots, n, \\
\delta r_{i j}+\left.\mathrm{d} K_{i j}\right|_{(t, x, \Phi(x))}(\delta t \oplus \delta u \oplus \delta v)=0, \quad i, j & =1, \ldots, n,
\end{aligned}
$$

for $\delta t \in T_{t} \mathbb{C}, \delta u \in T_{x} U, \delta v \in T_{\Phi(x)} V$, and $\left(\delta r_{i j}\right)_{i, j=1}^{n} \in T_{(0)_{i, j=1}^{n}} \mathbb{C}^{n^{2}}$, where we have used $\mathrm{d} x_{i}^{\prime}=\mathrm{d} y_{i}^{\prime}$ and $I_{j}=K_{i j}=0$ at $(t, x, \Phi(x))$. As $\left.\mathrm{d} y_{1}\right|_{x}, \ldots,\left.\mathrm{d} y_{n}\right|_{x}$ are a basis for $T_{x}^{*} U$, equations (3.30) are transverse, so $W$ is smooth of dimension $n+1$ near $\iota(t)$. Hence, taking $T$ small enough, we can suppose $W$ is smooth.

It remains to prove Proposition 3.4(a)-(e). Part (a) is immediate from (3.29). For (b), the vector space of solutions $\left(\delta t, \delta u, \delta v,\left(\delta r_{i j}\right)_{i, j=1}^{n}\right)$ to (3.30) is $T_{\iota(t)} W$, where $\left.\mathrm{d}\left(\pi_{\mathbb{C}} \times \pi_{U}\right)\right|_{\iota(t)}: T_{\iota(t)} W \rightarrow T_{(t, x)}(\mathbb{C} \times U)$ and $\left.\mathrm{d}\left(\pi_{\mathbb{C}} \times \pi_{V}\right)\right|_{\iota(t)}: T_{\iota(t)} W \rightarrow$ $T_{(t, \Phi(x))}(\mathbb{C} \times V)$ map $\left(\delta t, \delta u, \delta v,\left(\delta r_{i j}\right)_{i, j=1}^{n}\right)$ to $(\delta t, \delta u)$ and $(\delta t, \delta v)$. By (3.30), these are isomorphisms, so $\pi_{\mathbb{C}} \times \pi_{U}$ and $\pi_{\mathbb{C}} \times \pi_{V}$ are étale near $\iota(\mathbb{C})$. Making $T, W$ smaller, we can suppose $\pi_{\mathbb{C}} \times \pi_{U}$ and $\pi_{\mathbb{C}} \times \pi_{V}$ are étale. 
For (c), we have

$$
\begin{aligned}
(f \circ & \left.\pi_{W}-g \circ \pi_{U}\right)\left(t, u, v,\left(r_{i j}\right)_{i, j=1}^{n}\right)=f(u)-g(v)=\left(f \circ \pi_{U}-g \circ \pi_{V}\right)(t, u, v) \\
& =\sum_{i=1}^{n} I_{i} \cdot\left(\left(1-t^{\prime}\right) x_{i}^{\prime}+t^{\prime} y_{i}^{\prime}-z_{i}^{\prime}\right)+t^{\prime}\left(1-t^{\prime}\right) \sum_{i, j=1}^{n} K_{i j} I_{i} I_{j} \\
& +\sum_{i, j=1}^{n} L_{i j} \cdot\left(\left(1-t^{\prime}\right) x_{i}^{\prime}+t^{\prime} y_{i}^{\prime}-z_{i}^{\prime}\right)\left(\left(1-t^{\prime}\right) x_{j}^{\prime}+t^{\prime} y_{j}^{\prime}-z_{j}^{\prime}\right) \\
& =\sum_{i=1}^{n} I_{i} \cdot\left(\sum_{j=1}^{n} r_{i j} \cdot I_{j}\right)+t^{\prime}\left(1-t^{\prime}\right) \sum_{i, j=1}^{n} K_{i j} I_{i} I_{j} \\
& +\sum_{i, j=1}^{n} L_{i j} \cdot\left(\sum_{k=1}^{n} r_{i k} \cdot I_{k}\right)\left(\sum_{l=1}^{n} r_{j l} \cdot I_{l}\right) \\
& =\sum_{i, j=1}^{n} I_{i} I_{j} \cdot\left[r_{i j}+t^{\prime}\left(1-t^{\prime}\right) K_{i j}+\sum_{k, l=1}^{n} L_{k l} \cdot r_{k i} r_{l j}\right]=0,
\end{aligned}
$$

using (3.27) in the third step, the first equation of (3.28) in the fourth, rearranging and exchanging labels $i, k$ and $j, l$ in the fifth, and the second equation of (3.28) in the sixth. Hence $f \circ \pi_{U}-g \circ \pi_{V}=0: W \rightarrow \mathbb{C}$, proving (c).

For (d), from (3.28) we can show that $\left(\mathbb{C} \times(\right.$ id $\left.\times \Phi)(X) \times \mathbb{C}^{n^{2}}\right) \cap W$ is open and closed in $Z=\operatorname{Crit}(h)$, and contains $\iota(\mathbb{C})$. So making $T, W$ smaller we can take $Z=\left(\mathbb{C} \times(\right.$ id $\left.\times \Phi)(X) \times \mathbb{C}^{n^{2}}\right) \cap W$, and then (d) follows as $\left.\Phi\right|_{X}=\left.\Psi\right|_{X}$.

For (e), observe that when $t=0$ in (3.28), the second equation reduces to $r_{i j}=0$ near $\iota(\mathbb{C})$ as $t(1-t) K_{i j}(t, u, v)=0$, so making $T, W$ smaller gives

$$
\begin{aligned}
W_{0} & =\left\{\left(0, u, v,(0)_{i, j=1}^{n}\right) \in T:\left(x_{i}^{\prime}-z_{i}^{\prime}\right)(0, u, v)=0, i=1, \ldots, n\right\} \\
& =\left\{\left(0, u, v,(0)_{i, j=1}^{n}\right) \in T: v=\Phi(u)\right\} .
\end{aligned}
$$

Hence $\left.\Phi \circ \pi_{W}\right|_{W_{0}}=\left.\pi_{U}\right|_{W_{0}}$. Similarly, when $t=1$ we have

$$
\begin{aligned}
W_{1} & =\left\{\left(1, u, v,(0)_{i, j=1}^{n}\right) \in T:\left(y_{i}^{\prime}-z_{i}^{\prime}\right)(0, u, v)=0, i=1, \ldots, n\right\} \\
& =\left\{\left(0, u, v,(0)_{i, j=1}^{n}\right) \in T: v=\Psi(u)\right\},
\end{aligned}
$$

so that $\left.\Psi \circ \pi_{W}\right|_{W_{1}}=\left.\pi_{U}\right|_{W_{1}}$. This proves (e), and Proposition 3.4

3.2 Part (a): $\operatorname{det}\left(\left.\left.\mathrm{d} \Psi\right|_{X^{\text {red }}} ^{-1} \circ \mathrm{d} \Phi\right|_{X^{\text {red }}}\right)= \pm 1$

We work in the situation of Theorem 3.1. For each $x \in X \subseteq U$, consider the diagram of linear maps of vector spaces:

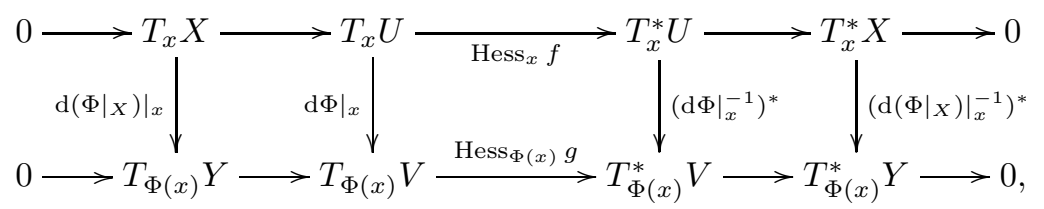


where $T_{x} X$ is the Zariski tangent space of $X$, and $\operatorname{Hess}_{x} f=\left.\left(\partial^{2} f\right)\right|_{x}$ the Hessian of $f$ at $x$. The rows of (3.31) are exact, and the columns isomorphisms. The outer squares of (3.31) clearly commute. We can show the central square commutes by taking second derivatives of $f=g \circ \Phi$ to get $\left.\partial^{2} f\right|_{x}=$ $\left.\partial^{2} g\right|_{\Phi(x)} \circ\left(\left.\left.\mathrm{d} \Phi\right|_{x} \otimes \mathrm{d} \Phi\right|_{x}\right)$, and composing with id $\left.\otimes \mathrm{d} \Phi\right|_{x} ^{-1}$. Thus (3.31) is commutative.

There is also an analogue of (3.31) for $\Psi$. Since $\Psi(x)=\Phi(x)$, we may compose the columns of (3.31) for $\Phi$ with the inverses of the columns of (3.31) for $\Psi$ to get a commutative diagram

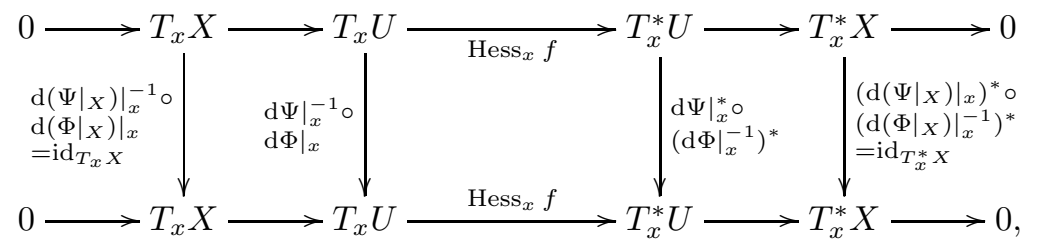

where the outer morphisms are identities as $\left.\Phi\right|_{X}=\left.\Psi\right|_{X}$.

Choose a complementary vector subspace $N_{x}$ to $T_{x} X$ in $T_{x} U$, which we think of as the normal to $X$ in $U$ at $x$, so that $T_{x} U=T_{x} X \oplus N_{x}$. Write $\operatorname{Hess}_{x}^{\prime} f$ for the restriction of $\operatorname{Hess}_{x} f$ to a symmetric bilinear form on $N_{x}$. Since $T_{x} X=\operatorname{Ker}\left(\operatorname{Hess}_{x} f\right)$, we see that $\operatorname{Hess}_{x}^{\prime} f$ is a nondegenerate symmetric bilinear form on $N_{x}$. We may write equation (3.32) as

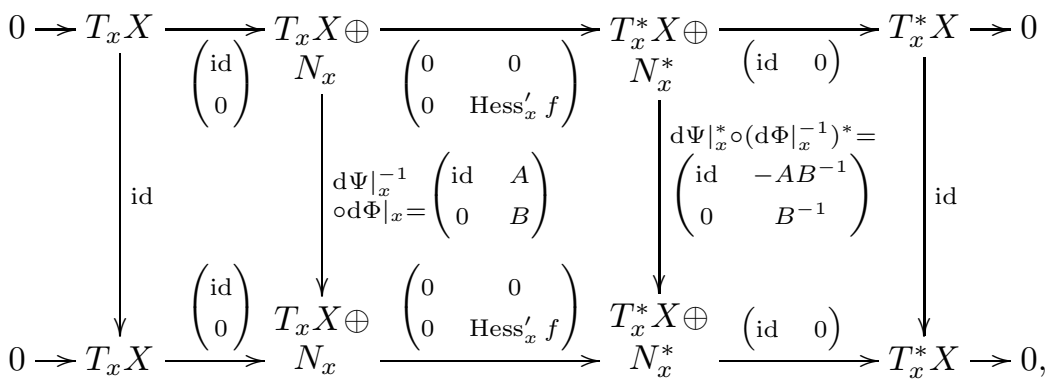

for some linear $A: N_{x} \rightarrow T_{x} X$ and $B: N_{x} \rightarrow N_{x}$. Then (3.33) commuting implies that $B$ preserves the nondegenerate symmetric bilinear form $\operatorname{Hess}_{x}^{\prime} f$ on $N_{x}$, and $\operatorname{det} B= \pm 1$. So $\operatorname{det}\left(\left.\left.\mathrm{d} \Psi\right|_{x} ^{-1} \circ \mathrm{d} \Phi\right|_{x}\right)=\operatorname{det}\left(\begin{array}{cc}\text { id } & A \\ 0 & B\end{array}\right)=\operatorname{det} B= \pm 1$ for $x \in X$.

Thus, as a map of topological spaces, $\operatorname{det}\left(\left.\left.\mathrm{d} \Psi\right|_{X^{\text {red }}} ^{-1} \circ \mathrm{d} \Phi\right|_{X^{\text {red }}}\right): X^{\text {red }} \rightarrow \mathbb{C} \backslash\{0\}$ actually maps $X^{\text {red }} \rightarrow\{ \pm 1\}$. Since it is continuous, it is locally constant. Now if $f, g: Y \rightarrow Z$ are morphisms of $\mathbb{C}$-schemes with $Y$ reduced, then $f=g$ if and only if $f(y)=g(y)$ for each point $y \in Y$. Applying this to compare $\operatorname{det}\left(\left.\left.\mathrm{d} \Psi\right|_{X^{\text {red }}} ^{-1} \circ \mathrm{d} \Phi\right|_{X^{\text {red }}}\right): X^{\text {red }} \rightarrow \mathbb{C} \backslash\{0\}$ locally with the constant maps 1 or -1 on $X^{\text {red }}$ shows that $\operatorname{det}\left(\left.\left.\mathrm{d} \Psi\right|_{X^{\text {red }}} ^{-1} \circ \mathrm{d} \Phi\right|_{X^{\text {red }}}\right)$ is a locally constant map $X^{\text {red }} \rightarrow\{ \pm 1\} \subset \mathbb{C} \backslash\{0\}$ as a $\mathbb{C}$-scheme morphism. This proves Theorem 3.1(a).

3.3 Part (b): $\mathcal{P} \mathcal{V}_{\Phi}=\operatorname{det}\left(\left.\left.\mathrm{d} \Psi\right|_{X^{\text {red }}} ^{-1} \circ \mathrm{d} \Phi\right|_{X^{\mathrm{red}}}\right) \cdot \mathcal{P} \mathcal{V}_{\Psi}$

For Theorem 3.1(b), we begin with the following proposition. 
Proposition 3.5. Let $U, V, \Phi, \Psi, f, g, X, Y$ be as in Theorem 3.1, and suppose $x \in X$ with $\left(\left.\left.\mathrm{d} \Psi\right|_{x} ^{-1} \circ \mathrm{d} \Phi\right|_{x}-\mathrm{id}_{T_{x} U}\right)^{2}=0$. Then there exists a Zariski open neighbourhood $X^{\prime}$ of $x$ in $X$ such that $\left.\mathcal{P} \mathcal{V}_{\Phi}\right|_{X^{\prime}}=\left.\mathcal{P} \mathcal{V}_{\Psi}\right|_{X^{\prime}}$.

Proof. Apply Proposition 3.4 to get $W, \pi_{\mathbb{C}}, \pi_{U}, \pi_{V}, \iota, h, Z$. Then apply Proposition 2.8 with $Z, X, x,\left.\pi_{\mathbb{C}}\right|_{Z},\left.\pi_{U}\right|_{Z}, \iota, \mathcal{P} \mathcal{V}_{U, f}^{\bullet},\left.\Phi\right|_{X} ^{*}\left(\mathcal{P} \mathcal{V}_{V, g}^{\bullet}\right), \mathcal{P} \mathcal{V}_{\Phi}, \mathcal{P} \mathcal{V}_{\Psi}$ in place of $W, X, x, \pi_{\mathbb{C}}, \pi_{X}, \iota, \mathcal{P}^{\bullet}, \mathcal{Q}^{\bullet}, \alpha, \beta$, respectively, and with $\gamma$ defined by the commuting diagram of isomorphisms:

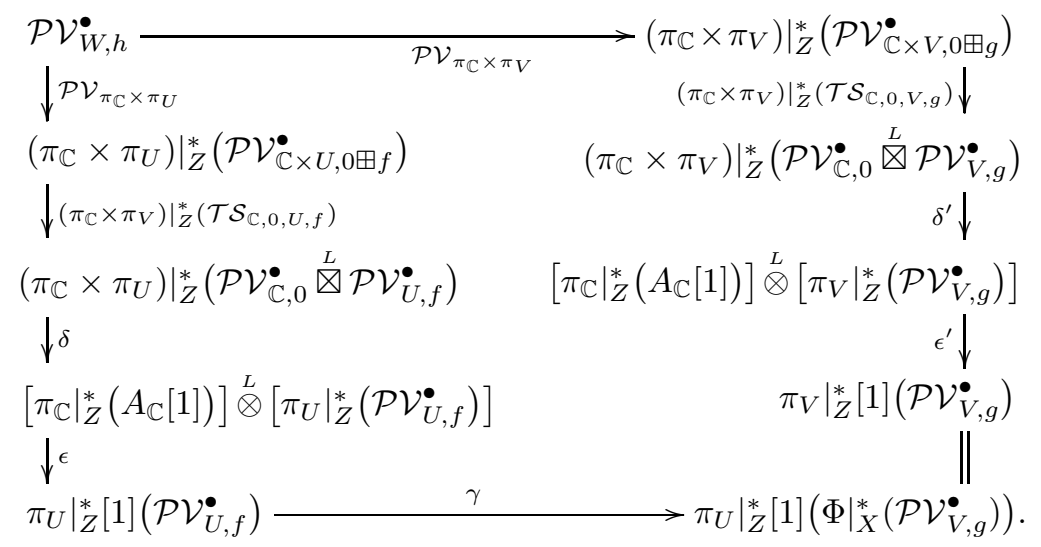

Here $\mathcal{T} \mathcal{S}_{\mathbb{C}, 0, U, f}, \mathcal{T} \mathcal{S}_{\mathbb{C}, 0, V, g}$ are as in ${ }_{L}(2.8), \delta, \delta^{\prime}$ come from $\mathcal{P} \mathcal{V}_{\mathbb{C}, 0} \cong A_{\mathbb{C}}[1]$, and $\epsilon, \epsilon^{\prime}$ from $\left.\pi_{\mathbb{C}}\right|_{Z} ^{*}\left(A_{\mathbb{C}}\right) \cong A_{Z}$ and $A_{Z} \otimes \mathcal{P}^{\bullet} \cong \mathcal{P}^{\bullet}$ for $\mathcal{P}^{\bullet} \in \operatorname{Perv}(Z)$.

Then the hypothesis $\left.\pi_{X}\right|_{W_{0}} ^{*}(\alpha)=j_{0}^{*}[-1](\gamma)$ in Proposition 2.8 follows from comparing $j_{0}^{*}[-1]$ applied to (3.34) with the commuting diagram

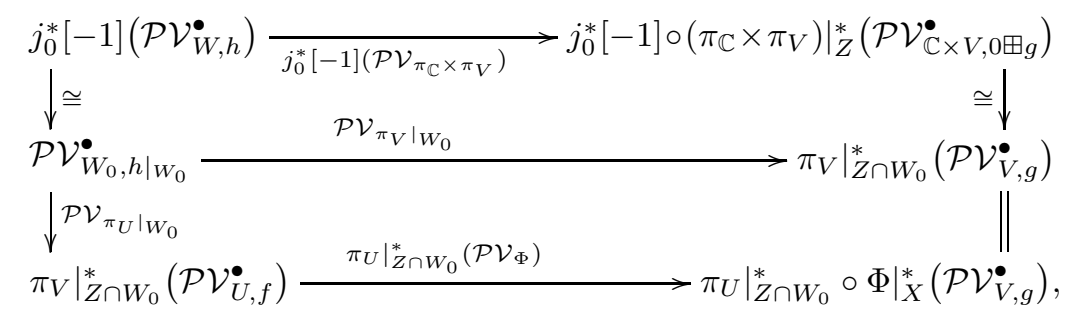

where $j_{0}: Z \cap W_{0} \hookrightarrow Z$ is the inclusion, and the bottom square commutes by Proposition 3.4(e) and (2.18). Similarly $\left.\pi_{X}\right|_{W_{1}} ^{*}(\beta)=j_{1}^{*}[-1](\gamma)$. Hence Proposition 2.8 gives Zariski open $x \in X^{\prime} \subseteq X$ with $\left.\mathcal{P} \mathcal{V}_{\Phi}\right|_{X^{\prime}}=\left.\mathcal{P} \mathcal{V}_{\Psi}\right|_{X^{\prime}}$.

Now to prove Theorem 3.1(b), let $x \in X$ be arbitrary. As in $\$ 3.2$, we can choose a splitting $T_{x} U=T_{x} X \oplus N_{x}$ such that

$$
\left.\left.\mathrm{d} \Psi\right|_{x} ^{-1} \circ \mathrm{d} \Phi\right|_{x}=\left(\begin{array}{cc}
\mathrm{id} & A \\
0 & B
\end{array}\right): \begin{gathered}
T_{x} X \oplus \\
N_{x}
\end{gathered} \longrightarrow \begin{gathered}
T_{x} X \oplus \\
N_{x}
\end{gathered}
$$

for linear $A: N_{x} \rightarrow T_{x} X$ and $B: N_{x} \rightarrow N_{x}$, where $B$ preserves the nondegenerate symmetric bilinear form $\operatorname{Hess}_{x}^{\prime} f$ on $N_{x}$. 
Choose a Zariski open neighbourhood $U^{\prime}$ of $x$ in $U$ and a splitting $T U^{\prime}=E \oplus$ $F$ for algebraic vector subbundles $E, F \subseteq T U$ with $\left.E\right|_{x}=T_{x} X$ and $\left.F\right|_{x}=N_{x}$. Then $\left.\mathrm{d} f\right|_{U^{\prime}}=\alpha \oplus \beta$ for unique $\alpha \in H^{0}(E)$ and $\beta \in H^{0}(F)$, and $X \cap U^{\prime}$ is defined by $\alpha=\beta=0$. Since $\operatorname{Hess}_{x} f=\left.\partial(\mathrm{d} f)\right|_{x}$ is nondegenerate on $N_{x}$, we see that $\left.\nabla \beta\right|_{x}:\left.T_{x} U \rightarrow F\right|_{x}$ induces an isomorphism $\left.N_{x} \rightarrow F\right|_{x}$, so $\left.\nabla \beta\right|_{x}$ is surjective. Therefore $S:=\beta^{-1}(0)$ is a smooth $\mathbb{C}$-subscheme of $U^{\prime}$ near $x$, and making $U^{\prime}$ smaller, we can suppose $S$ is smooth. Set $e=\left.f\right|_{S}: S \rightarrow \mathbb{C}$. Then the isomorphism $\left.T^{*} S \cong E\right|_{S}$ identifies de $\in H^{0}\left(T^{*} S\right)$ with $\left.\alpha\right|_{S} \in H^{0}\left(\left.E\right|_{S}\right)$. Hence $\operatorname{Crit}(e: S \rightarrow \mathbb{C})=\operatorname{Crit}\left(\left.f\right|_{U^{\prime}}: U^{\prime} \rightarrow \mathbb{C}\right)=X \cap U^{\prime}$, as $\mathbb{C}$-subschemes of $U$.

By [23. Prop. 2.23] quoted in Theorem 5.1(i) below, there exist a smooth $\mathbb{C}$-scheme $R$, morphisms $\gamma: R \rightarrow U^{\prime}, \delta: R \rightarrow S, \epsilon: R \rightarrow \mathbb{C}^{n}$ where $n=\operatorname{dim} U^{\prime}-$ $\operatorname{dim} S$, and $r \in R$, such that $\gamma(r)=x,\left.\gamma\right|_{Q}=\left.\delta\right|_{Q}, f \circ \gamma=e \circ \delta+\left(z_{1}^{2}+\cdots+z_{n}^{2}\right) \circ \epsilon$ : $R \rightarrow \mathbb{C}$, and the following commutes with horizontal morphisms étale:

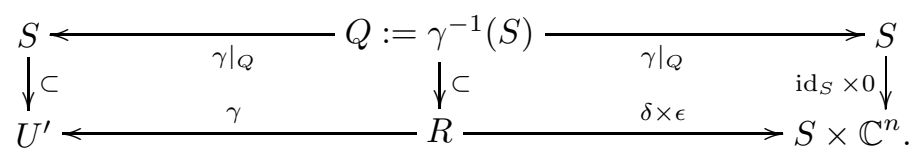

Taking derivatives at $r \in Q \subseteq R$ in (3.36) gives a commutative diagram

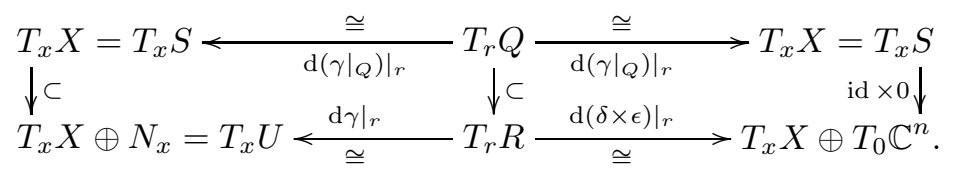

Therefore $\left.\left.\mathrm{d}(\delta \times \epsilon)\right|_{r} \circ \mathrm{d} \gamma\right|_{r} ^{-1}: T_{x} X \oplus N_{x} \rightarrow T_{x} X \oplus T_{0} \mathbb{C}^{n}$ is the identity on $T_{x} X$, and induces an isomorphism $N_{x} \rightarrow T_{0} \mathbb{C}^{n}$, which as $f \circ \gamma=e \circ \delta+\left(z_{1}^{2}+\cdots+z_{n}^{2}\right) \circ \epsilon$ identifies $\operatorname{Hess}_{x}^{\prime} f$ on $N_{x}$ with $\operatorname{Hess}_{0}\left(z_{1}^{2}+\cdots+z_{n}^{2}\right)=\mathrm{d} z_{1} \otimes \mathrm{d} z_{1}+\cdots+\mathrm{d} z_{n} \otimes \mathrm{d} z_{n}$ on $T_{0} \mathbb{C}^{n}$. Thus, the linear isomorphism $B: N_{x} \rightarrow N_{x}$ above preserving $\operatorname{Hess}_{x}^{\prime} f$ is identified with a linear isomorphism $M: \mathbb{C}^{n} \rightarrow \mathbb{C}^{n}$ preserving $\mathrm{d} z_{1} \otimes \mathrm{d} z_{1}+$ $\cdots+\mathrm{d} z_{n} \otimes \mathrm{d} z_{n}$, that is, $M \in \mathrm{O}(n, \mathbb{C})$ satisfies

$$
\left.\left.\left(\begin{array}{cc}
\text { id } & 0 \\
0 & B
\end{array}\right) \circ \mathrm{d} \gamma\right|_{r} \circ \mathrm{d}(\delta \times \epsilon)\right|_{r} ^{-1}=\left.\left.\mathrm{d} \gamma\right|_{r} \circ \mathrm{d}(\delta \times \epsilon)\right|_{r} ^{-1} \circ\left(\begin{array}{cc}
\text { id } & 0 \\
0 & M
\end{array}\right) \text {. }
$$

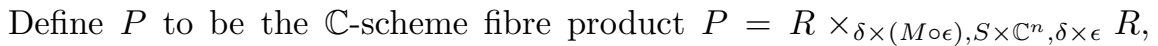
with projections $\pi_{1}, \pi_{2}: P \rightarrow R$. Then $P$ is smooth and $\pi_{1}, \pi_{2}$ are étale, as $R, S \times \mathbb{C}^{n}$ are smooth and $\delta \times(M \circ \epsilon), \delta \times \epsilon: R \rightarrow S \times \mathbb{C}^{n}$ are étale. As $r \in R$ with $(\delta \times(M \circ \epsilon))(r)=(x, 0)=(\delta \times \epsilon)(r)$, we have a point $p \in P$ with $\pi_{1}(p)=\pi_{2}(p)=r$. Define $d=f \circ \gamma \circ \pi_{1}: P \rightarrow \mathbb{C}$ and $Z=\operatorname{Crit}(d)$. Then

$$
\begin{aligned}
d & =f \circ \gamma \circ \pi_{1}=\left(e \boxplus z_{1}^{2}+\cdots+z_{n}^{2}\right) \circ(\delta \times \epsilon) \circ \pi_{1} \\
& =\left(e \boxplus z_{1}^{2}+\cdots+z_{n}^{2}\right) \circ(\delta \times(M \circ \epsilon)) \circ \pi_{1} \\
& =\left(e \boxplus z_{1}^{2}+\cdots+z_{n}^{2}\right) \circ(\delta \times \epsilon) \circ \pi_{2}=f \circ \gamma \circ \pi_{2} .
\end{aligned}
$$

Consider the étale morphisms $\Phi \circ \gamma \circ \pi_{1}, \Psi \circ \gamma \circ \pi_{2}: P \rightarrow V$. Both map $p \mapsto \Phi(x)$, and satisfy $g \circ\left(\Phi \circ \gamma \circ \pi_{1}\right)=d=g \circ\left(\Psi \circ \gamma \circ \pi_{2}\right)$ by (3.38) and 
$g \circ \Phi=f=g \circ \Psi$. Taking derivatives at $p$ to get linear maps $T_{p} P \rightarrow T_{\Phi(x)} V$, we find that

$$
\begin{aligned}
\left.\mathrm{d}\left(\Psi \circ \gamma \circ \pi_{2}\right)\right|_{p}=\left.\left.\left.\left.\mathrm{d} \Psi\right|_{x} \circ \mathrm{d} \gamma\right|_{r} \circ \mathrm{d}(\delta \times \epsilon)\right|_{r} ^{-1} \circ \mathrm{d}\left((\delta \times \epsilon) \circ \pi_{2}\right)\right|_{p} \\
\quad=\left.\left.\left.\left.\mathrm{d} \Psi\right|_{x} \circ \mathrm{d} \gamma\right|_{r} \circ \mathrm{d}(\delta \times \epsilon)\right|_{r} ^{-1} \circ \mathrm{d}\left((\delta \times(M \circ \epsilon)) \circ \pi_{1}\right)\right|_{p} \\
\quad=\left.\left.\left.\left.\left.\mathrm{d} \Psi\right|_{x} \circ \mathrm{d} \gamma\right|_{r} \circ \mathrm{d}(\delta \times \epsilon)\right|_{r} ^{-1} \circ\left(\begin{array}{cc}
\text { id } & 0 \\
0 & M
\end{array}\right) \circ \mathrm{d}(\delta \times \epsilon)\right|_{r} \circ \mathrm{d} \pi_{1}\right|_{p} \\
\quad=\left.\left.\left.\left.\left.\mathrm{d} \Psi\right|_{x} \circ\left(\begin{array}{cc}
\text { id } & 0 \\
0 & B
\end{array}\right) \circ \mathrm{d} \gamma\right|_{r} \circ \mathrm{d}(\delta \times \epsilon)\right|_{r} ^{-1} \circ \mathrm{d}(\delta \times \epsilon)\right|_{r} \circ \mathrm{d} \pi_{1}\right|_{p} \\
\quad=\left.\left.\left.\mathrm{d} \Psi\right|_{x} \circ\left(\begin{array}{cc}
\text { id } & -A B^{-1} \\
0 & \text { id }
\end{array}\right)\left(\begin{array}{cc}
\text { id } & A \\
0 & B
\end{array}\right) \circ \mathrm{d} \gamma\right|_{r} \circ \mathrm{d} \pi_{1}\right|_{p} \\
\quad=\left.\left.\left.\mathrm{d} \Psi\right|_{x} \circ\left(\begin{array}{cc}
\text { id } & -A B^{-1} \\
0 & \text { id }
\end{array}\right) \circ \mathrm{d} \Psi\right|_{x} ^{-1} \circ \mathrm{d}\left(\Phi \circ \gamma \circ \pi_{1}\right)\right|_{p},
\end{aligned}
$$

using $(\delta \times(M \circ \epsilon)) \circ \pi_{1}=(\delta \times \epsilon) \circ \pi_{2}$ in the second step, (3.37) in the fourth, and (3.35) in the sixth. Since $\left[\left(\begin{array}{c}\mathrm{id}-A B^{-1} \\ 0\end{array}\right)-\mathrm{id}\right]^{2}=0$, equation (3.39) implies that $\left(\left.\left.\mathrm{d}\left(\Psi \circ \gamma \circ \pi_{2}\right)\right|_{p} ^{-1} \circ \mathrm{d}\left(\Phi \circ \gamma \circ \pi_{1}\right)\right|_{p}-\mathrm{id}_{T_{p} P}\right)^{2}=0$, and thus Proposition 3.5 gives a Zariski open neighbourhood $P^{\prime}$ of $p$ in $P$ such that

$$
\left.\mathcal{P} \mathcal{V}_{\Phi \circ \gamma \circ \pi_{1}}\right|_{P^{\prime}}=\left.\mathcal{P} \mathcal{V}_{\Psi \circ \gamma \circ \pi_{2}}\right|_{P^{\prime}}:\left.\left.\left.\mathcal{P} \mathcal{V}_{P, d}^{\bullet}\right|_{P^{\prime}} \longrightarrow\left(\Phi \circ \gamma \circ \pi_{1}\right)\right|_{Z} ^{*}\left(\mathcal{P} \mathcal{V}_{V, g}^{\bullet}\right)\right|_{P^{\prime}}
$$

Since $(\delta \times(M \circ \epsilon)) \circ \pi_{1}=(\delta \times \epsilon) \circ \pi_{2}: P \rightarrow S \times \mathbb{C}^{n}$ are étale with $\left(e \boxplus z_{1}^{2}+\cdots+z_{n}^{2}\right) \circ(\delta \times(M \circ \epsilon)) \circ \pi_{1}=d=\left(e \boxplus z_{1}^{2}+\cdots+z_{n}^{2}\right) \circ(\delta \times \epsilon) \circ \pi_{2}$, we see using (2.8) and (2.18) that

$$
\begin{aligned}
\left.\pi_{1}\right|_{Z} ^{*}[ & \left.\mathcal{P} \mathcal{V}_{\delta} \stackrel{L}{\otimes}\left(\left.M\right|_{\{0\}} ^{*}\left(\mathcal{P} \mathcal{V}_{\epsilon}\right) \circ \mathcal{P} \mathcal{V}_{M}\right)\right] \circ \mathcal{P} \mathcal{V}_{\pi_{1}}=\left.\pi_{1}\right|_{Z} ^{*}\left[\mathcal{P} \mathcal{V}_{\delta}{ }^{L} \mathcal{P} \mathcal{V}_{M \circ \epsilon}\right] \circ \mathcal{P} \mathcal{V}_{\pi_{1}} \\
& \left.\cong \pi_{1}\right|_{Z} ^{*}\left(\mathcal{P} \mathcal{V}_{\delta \times(M \circ \epsilon)}\right) \circ \mathcal{P} \mathcal{V}_{\pi_{1}}=\mathcal{P} \mathcal{V}_{(\delta \times(M \circ \epsilon)) \circ \pi_{1}}=\mathcal{P} \mathcal{V}_{(\delta \times \epsilon) \circ \pi_{2}} \\
& =\left.\left.\pi_{2}\right|_{Z} ^{*}\left(\mathcal{P} \mathcal{V}_{\delta \times \epsilon}\right) \circ \mathcal{P} \mathcal{V}_{\pi_{2}} \cong \pi_{2}\right|_{Z} ^{*}\left[\mathcal{P} \mathcal{V}_{\delta} \stackrel{L}{\otimes} \mathcal{P} \mathcal{V}_{\epsilon}\right] \circ \mathcal{P} \mathcal{V}_{\pi_{2}}
\end{aligned}
$$

where ' $\cong$ ' are equalities after identifying both sides of (2.8). Since $\left.\pi_{1}\right|_{Z}=\left.\pi_{2}\right|_{Z}$, and $\left.M\right|_{\{0\}}=\operatorname{id}_{\{0\}}$, and Example 2.16 shows that $\mathcal{P} \mathcal{V}_{M}$ in (2.19) is multiplication by $\operatorname{det} M$, equation (3.41) implies that

$$
\left.\operatorname{det} M \cdot \pi_{1}\right|_{Z} ^{*}\left[\mathcal{P} \mathcal{V}_{\delta} \stackrel{L}{\otimes} \mathcal{P} \mathcal{V}_{\epsilon}\right] \circ \mathcal{P} \mathcal{V}_{\pi_{1}}=\left.\pi_{1}\right|_{Z} ^{*}\left[\mathcal{P} \mathcal{V}_{\delta} \stackrel{L}{\otimes} \mathcal{P} \mathcal{V}_{\epsilon}\right] \circ \mathcal{P} \mathcal{V}_{\pi_{2}}
$$

As $\left.\pi_{1}\right|_{Z} ^{*}\left[\mathcal{P} \mathcal{V}_{\delta} \otimes^{L} \mathcal{P} \mathcal{V}_{\epsilon}\right]$ is an isomorphism, this gives

$$
\operatorname{det} M \cdot \mathcal{P} \mathcal{V}_{\pi_{1}}=\mathcal{P} \mathcal{V}_{\pi_{2}}:\left.\mathcal{P} \mathcal{V}_{P, d}^{\bullet} \longrightarrow \pi_{1}\right|_{Z} ^{*}\left(\mathcal{P} \mathcal{V}_{R, e}^{\bullet}\right)
$$

Writing $Z^{\prime}=Z \cap P^{\prime}$, we now have

$$
\begin{aligned}
\left.\left(\gamma \circ \pi_{1}\right)\right|_{Z^{\prime}} ^{*}\left(\mathcal{P} \mathcal{V}_{\Phi}\right) \circ \mathcal{P} \mathcal{V}_{\gamma \circ \pi_{1}}\left|P^{\prime}=\mathcal{P} \mathcal{V}_{\Phi \circ \gamma \circ \pi_{1}}\right| P_{P^{\prime}}=\left.\mathcal{P} \mathcal{V}_{\Psi \circ \gamma \circ \pi_{2}}\right|_{P^{\prime}} \\
\quad=\left.\pi_{2}\right|_{Z^{\prime}} ^{*}\left(\mathcal{P} \mathcal{V}_{\Psi \circ \gamma}\right) \circ \mathcal{P} \mathcal{V}_{\pi_{2}} \mid P^{\prime} \\
\quad=\left.\left.\operatorname{det} M \cdot \pi_{1}\right|_{Z^{\prime}} ^{*}\left(\mathcal{P} \mathcal{V}_{\Psi \circ \gamma}\right) \circ \mathcal{P} \mathcal{V}_{\pi_{1}}\right|_{P^{\prime}}=\left.\operatorname{det} M \cdot \mathcal{P} \mathcal{V}_{\Psi \circ \gamma \circ \pi_{1}}\right|_{P^{\prime}} \\
\quad=\left.\operatorname{det} M \cdot\left(\gamma \circ \pi_{1}\right)\right|_{Z^{\prime}} ^{*}\left(\mathcal{P} \mathcal{V}_{\Psi}\right) \circ \mathcal{P} \mathcal{V}_{\gamma \circ \pi_{1}} \mid P_{P^{\prime}},
\end{aligned}
$$


using (3.40) in the second step, (3.42) and $\left.\pi_{1}\right|_{Z^{\prime}}=\left.\pi_{2}\right|_{Z^{\prime}}$ in the fourth, and (2.18) in the rest. As $\left.\mathcal{P} \mathcal{V}_{\gamma \circ \pi_{1}}\right|_{P^{\prime}}$ is an isomorphism, (3.43) implies that

$$
\left.\left(\gamma \circ \pi_{1}\right)\right|_{Z^{\prime}} ^{*}\left(\mathcal{P} \mathcal{V}_{\Phi}\right)=\left.\operatorname{det} M \cdot\left(\gamma \circ \pi_{1}\right)\right|_{Z^{\prime}} ^{*}\left(\mathcal{P} \mathcal{V}_{\Psi}\right)
$$

and by Theorem 2.7(i) this implies that

$$
\left.\mathcal{P} \mathcal{V}_{\Phi}\right|_{X^{\prime}}=\left.\operatorname{det} M \cdot \mathcal{P} \mathcal{V}_{\Phi}\right|_{X^{\prime}}
$$

where $X^{\prime}=\left(\gamma \circ \pi_{1}\right)\left(Z^{\prime}\right)$ is a Zariski open neighbourhood of $x$ in $X$, since $\left.\left(\gamma \circ \pi_{1}\right)\right|_{Z^{\prime}}: Z^{\prime} \rightarrow X$ is étale with $\gamma \circ \pi_{1}(p)=x$. Now (3.35) and (3.37) give $\operatorname{det}\left(\left.\left.\mathrm{d} \Psi\right|_{x} ^{-1} \circ \mathrm{d} \Phi\right|_{x}\right)=\operatorname{det}\left(\begin{array}{cc}\mathrm{id} & A \\ 0 & B\end{array}\right)=\operatorname{det} B=\operatorname{det} M$. So (3.44) proves that (3.1) holds near $x$ in $X$. As this is true for all $x \in X$, Theorem 3.1(b) follows.

\section{$3.4 \mathscr{D}$-modules and mixed Hodge modules}

The proof of Proposition 3.4 applies verbatim also in the analytic context. Theorem 3.1 (a),(b) then follow from Proposition 3.4 and the argument given above, using 42.5 including the Sheaf Property (x) for morphisms. Hence all these results carry over to our other contexts $2.6-2.10$.

\section{Dependence of $\mathcal{P} \mathcal{V}_{U, f}^{\bullet}$ on $f$}

We will use the following notation:

Definition 4.1. Let $U$ be a smooth $\mathbb{C}$-scheme, $f: U \rightarrow \mathbb{C}$ a regular function, and $X=\operatorname{Crit}(f)$ as a closed $\mathbb{C}$-subscheme of $U$. Write $I_{X} \subseteq \mathcal{O}_{U}$ for the sheaf of ideals of regular functions $U \rightarrow \mathbb{C}$ vanishing on $X$, so that $I_{X}=I_{\mathrm{d} f}$. For each $k=1,2, \ldots$, write $X^{(k)}$ for the $k^{\text {th }}$ order thickening of $X$ in $U$, that is, $X^{(k)}$ is the closed $\mathbb{C}$-subscheme of $U$ defined by the sheaf of ideals $I_{X}^{k}$ in $\mathcal{O}_{U}$. Also write $X^{\text {red }}$ for the reduced $\mathbb{C}$-subscheme of $U$.

Then we have a chain of inclusions of closed $\mathbb{C}$-subschemes

$$
X^{\mathrm{red}} \subseteq X=X^{(1)} \subseteq X^{(2)} \subseteq X^{(3)} \subseteq \cdots \subseteq U .
$$

Write $f^{(k)}:=\left.f\right|_{X^{(k)}}: X^{(k)} \rightarrow \mathbb{C}$, and $f^{\text {red }}:=\left.f\right|_{X^{\text {red }}}: X^{\text {red }} \rightarrow \mathbb{C}$, so that $f^{(k)}, f^{\text {red }}$ are regular functions on the $\mathbb{C}$-schemes $X^{(k)}, X^{\text {red }}$. Note that $f^{\text {red }}$ : $X^{\text {red }} \rightarrow \mathbb{C}$ is locally constant, since $X=\operatorname{Crit}(f)$.

We also use the same notation for complex analytic spaces.

In $\$ 2.4$ we defined the perverse sheaf of vanishing cycles $\mathcal{P} \mathcal{V}_{U, f}^{\bullet}$ in $\operatorname{Perv}(X)$. So we can ask: how much of the sequence (4.1) does $\mathcal{P} \mathcal{V}_{U, f}^{\bullet}$ depend on? That is, is $\mathcal{P} \mathcal{V}_{U, f}^{\bullet}$ (canonically?) determined by $\left(X^{\text {red }}, f^{\text {red }}\right.$ ), or by $\left(X^{(k)}, f^{(k)}\right)$ for some $k \geq 1$, as well as by $(U, f)$ ? Our next theorem shows that $\mathcal{P} \mathcal{V}_{U, f}^{\bullet}$ is determined up to canonical isomorphism by $\left(X^{(3)}, f^{(3)}\right)$, and hence a fortiori also by $\left(X^{(k)}, f^{(k)}\right)$ for $k>3$ : 
Theorem 4.2. Let $U, V$ be smooth $\mathbb{C}$-schemes, $f: U \rightarrow \mathbb{C}, g: V \rightarrow \mathbb{C}$ be regular functions, and $X=\operatorname{Crit}(f), Y=\operatorname{Crit}(g)$ as closed $\mathbb{C}$-subschemes of $U, V$, so that 2.4 defines perverse sheaves $\mathcal{P} \mathcal{V}_{U, f}^{\bullet}, \mathcal{P} \mathcal{V}_{V, g}^{\bullet}$ on $X, Y$. Define $X^{(3)}, f^{(3)}$ and $Y^{(3)}, g^{(3)}$ as in Definition 4.1, and suppose $\Phi: X^{(3)} \rightarrow Y^{(3)}$ is an isomorphism with $g^{(3)} \circ \Phi=f^{(3)}$, so that $\left.\Phi\right|_{X}: X \rightarrow Y \subseteq Y^{(3)}$ is an isomorphism.

Then there is a canonical isomorphism in $\operatorname{Perv}(X)$

$$
\Omega_{\Phi}:\left.\mathcal{P} \mathcal{V}_{U, f}^{\bullet} \longrightarrow \Phi\right|_{X} ^{*}\left(\mathcal{P} \mathcal{V}_{V, g}^{\bullet}\right)
$$

which is characterized by the property that if $T$ is a smooth $\mathbb{C}$-scheme and $\pi_{U}: T \rightarrow U, \pi_{V}: T \rightarrow V$ are étale morphisms with $e:=f \circ \pi_{U}=g \circ \pi_{V}:$ $T \rightarrow \mathbb{C}$, so that $\left.\pi_{U}\right|_{Q}: Q \rightarrow X,\left.\pi_{V}\right|_{Q}: Q \rightarrow Y$ are étale for $Q:=\operatorname{Crit}(e)$, and $\left.\Phi \circ \pi_{U}\right|_{Q^{(2)}}=\left.\pi_{V}\right|_{Q^{(2)}}: Q^{(2)} \rightarrow Y^{(2)}$, then

$$
\left.\pi_{U}\right|_{Q} ^{*}\left(\Omega_{\Phi}\right) \circ \mathcal{P} \mathcal{V}_{\pi_{U}}=\mathcal{P} \mathcal{V}_{\pi_{V}}:\left.\mathcal{P} \mathcal{V}_{T, e}^{\bullet} \longrightarrow \pi_{V}\right|_{Q} ^{*}\left(\mathcal{P} \mathcal{V}_{U, f}^{\bullet}\right)
$$

Also the following commute, where $\sigma_{U, f}, \sigma_{V, g}, \tau_{U, f}, \tau_{V, g}$ are as in (2.6)-(2.7):

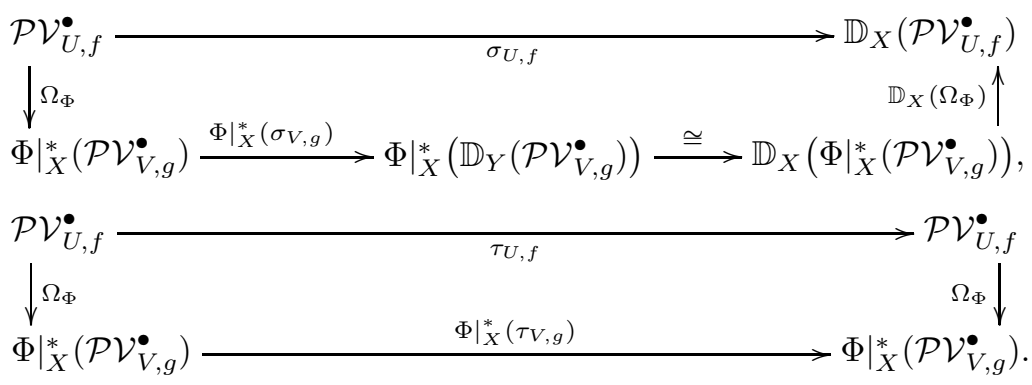

If there exists an étale morphism $\Xi: U \rightarrow V$ with $g \circ \Xi=f: U \rightarrow \mathbb{C}$ and $\left.\Xi\right|_{X^{(3)}}=\Phi: X^{(3)} \rightarrow Y^{(3)}$ then $\Omega_{\Phi}=\mathcal{P} \mathcal{V}_{\Xi}$, for $\mathcal{P} \mathcal{V}_{\Xi}$ as in (2.14).

If $W$ is another smooth $\mathbb{C}$-scheme, $h: W \rightarrow \mathbb{C}$ is regular, $Z=\operatorname{Crit}(h)$, and $\Psi: Y^{(3)} \rightarrow Z^{(3)}$ is an isomorphism with $h^{(3)} \circ \Psi=g^{(3)}$ then

$$
\Omega_{\Psi \circ \Phi}=\left.\Phi\right|_{X} ^{*}\left(\Omega_{\Psi}\right) \circ \Omega_{\Phi}:\left.\mathcal{P} \mathcal{V}_{U, f}^{\bullet} \longrightarrow(\Psi \circ \Phi)\right|_{X} ^{*}\left(\mathcal{P} \mathcal{V}_{W, h}^{\bullet}\right) .
$$

If $U=V, f=g, X=Y$ and $\Phi=\operatorname{id}_{X^{(3)}}$ then $\Omega_{\mathrm{id}_{X^{(3)}}}=\mathrm{id}_{\mathcal{P} V_{U, f}^{\bullet}}$.

The analogues of all the above also hold with appropriate modifications for $\mathscr{D}$ modules on $\mathbb{C}$-schemes, for perverse sheaves and $\mathscr{D}$-modules on complex analytic spaces, and for mixed Hodge modules on $\mathbb{C}$-schemes and complex analytic spaces, as in 2.6 2.10.

We will prove Theorem 4.2 in 4.24 .3 . The proof for $\mathbb{C}$-schemes depends on the case $k=2$ of the following proposition, proved in $\$ 4.1$

Proposition 4.3. Let $U, V$ be smooth $\mathbb{C}$-schemes, $f: U \rightarrow \mathbb{C}, g: V \rightarrow \mathbb{C}$ be regular functions, and $X=\operatorname{Crit}(f) \subseteq U, Y=\operatorname{Crit}(g) \subseteq V$. Using the notation of Definition 4.1, suppose $\Phi: \bar{X}^{(k+1)} \rightarrow Y^{(k+1)}$ is an isomorphism with $g^{(k+1)} \circ \Phi=f^{(k+1)}$ for some $k \geqslant 2$. Then for each $x \in X$ we can choose a smooth $\mathbb{C}$-scheme $T$ and étale morphisms $\pi_{U}: T \rightarrow U, \pi_{V}: T \rightarrow V$ such that 
(a) $e:=f \circ \pi_{U}=g \circ \pi_{V}: T \rightarrow \mathbb{C}$;

(b) setting $Q=\operatorname{Crit}(e)$, then $\left.\pi_{U}\right|_{Q^{(k)}}: Q^{(k)} \rightarrow X^{(k)} \subseteq U$ is an isomorphism with a Zariski open neighbourhood $\tilde{X}^{(k)}$ of $x$ in $X^{(k)}$; and

(c) $\left.\Phi \circ \pi_{U}\right|_{Q^{(k)}}=\left.\pi_{V}\right|_{Q^{(k)}}: Q^{(k)} \rightarrow Y^{(k)}$.

The proof of Proposition 4.3 is similar to that of Proposition 3.5 in 3.1 One can also prove an analogue of Proposition 4.3 when $k=1$, but in part (b) $\left.\pi_{U}\right|_{Q^{(1)}}: Q^{(1)} \rightarrow X^{(1)}$ must be étale rather than a Zariski open inclusion.

In Proposition 4.3, we start with $\Phi: X^{(k+1)} \stackrel{\cong}{\longrightarrow} Y^{(k+1)}$, but we construct $T, \pi_{U}, \pi_{V}$ with $\left.\Phi \circ \pi_{U}\right|_{Q^{(k)}}=\left.\pi_{V}\right|_{Q^{(k)}}$. One might expect to find $T, \pi_{U}, \pi_{V}$ with $\left.\Phi \circ \pi_{U}\right|_{Q^{(k+1)}}=\left.\pi_{V}\right|_{Q^{(k+1)}}$, but the next example shows this is not possible.

Example 4.4. Let $U, V$ be open neighbourhoods of 0 in $\mathbb{C}$, and $f: U \rightarrow$ $\mathbb{C}, g: V \rightarrow \mathbb{C}$ be regular functions given as power series by $f(x)=x^{m+1}$ and $g(y)=y^{m+1}+A y^{(k+1) m}+\cdots$, for $k, m \geqslant 2$ and $0 \neq A \in \mathbb{C}$, where 0 is the only critical point of $g$. Then $X:=\operatorname{Crit}(f)=\operatorname{Spec}\left(\mathbb{C}[x] /\left(x^{m}\right)\right)$ and $Y:=\operatorname{Crit}(g)=\operatorname{Spec}\left(\mathbb{C}[y] /\left(y^{m}\right)\right)$, so $X^{(k+1)}=\operatorname{Spec}\left(\mathbb{C}[x] /\left(x^{(k+1) m}\right)\right)$, $f^{(k+1)}=x^{m+1}+\left(x^{(k+1) m}\right)$, and $Y^{(k+1)}=\operatorname{Spec}\left(\mathbb{C}[y] /\left(y^{(k+1) m}\right)\right), g^{(k+1)}=$ $y^{m+1}+\left(y^{(k+1) m}\right)$. Thus $\Phi: X^{(k+1)} \rightarrow Y^{(k+1)}$ acing on functions by $y+$ $\left(y^{(k+1) m}\right) \mapsto x+\left(x^{(k+1) m}\right)$ is an isomorphism with $f^{(k+1)}=g^{(k+1)} \circ \Phi$.

Suppose $T, \pi_{U}, \pi_{V}$ are as in Proposition 4.3 and use $w=x \circ \pi_{U}$ as a coordinate on $T$. Then $e(w)=w^{m+1}$, and $Q=\operatorname{Crit}(e)=\operatorname{Spec}\left(\mathbb{C}[w] /\left(w^{m}\right)\right)$. We have $\pi_{U}(w)=w$, so $\Phi \circ \pi_{U}(w)=w$, but $w^{m+1}=\pi_{V}(w)^{m+1}+A \pi_{V}(w)^{(k+1) m}+\cdots$, so that $\pi_{V}(w)=w-\frac{1}{m+1} A w^{k m}+\cdots$. Thus, $\Phi \circ \pi_{U}: T \rightarrow V$ and $\pi_{V}: T \rightarrow V$ differ by $-\frac{1}{m+1} A w^{k m}+\cdots$, which is zero on $Q^{(k)}$ but not on $Q^{(k+1)}$. Hence in this example there do not exist $T, \pi_{U}, \pi_{V}$ with $\left.\Phi \circ \pi_{U}\right|_{Q^{(k+1)}}=\left.\pi_{V}\right|_{Q^{(k+1)}}$.

Remark 4.5. We can also ask: can we improve $\left(X^{(3)}, f^{(3)}\right)$ in Theorem 4.2 to $\left(X^{(2)}, f^{(2)}\right)$ or $\left(X^{(1)}, f^{(1)}\right)$ or $\left(X^{\text {red }}, f^{\text {red }}\right)$ ? Here are some thoughts on this.

(a) The analogue of Proposition 4.3 for $k=1$ mentioned above implies that étale or complex analytically locally on $X,(U, f)$ and hence $\mathcal{P} \mathcal{V}_{U, f}^{\bullet}$ are determined up to non-canonical isomorphism by $\left(X^{(2)}, f^{(2)}\right)$. Using the ideas of $95-\sqrt{6}$, one can show that these non-canonical isomorphisms of $\mathcal{P} \mathcal{V}_{U, f}^{\bullet}$ are unique up to sign.

(b) Consider the following example: let $U=(\mathbb{C} \backslash\{0\}) \times \mathbb{C}=V$, and define $f$ : $U \rightarrow \mathbb{C}$ and $g: V \rightarrow \mathbb{C}$ by $f(x, y)=y^{2}$ and $g(x, y)=x y^{2}$. Then $X:=\operatorname{Crit}(f)=$ $\{y=0\}=\operatorname{Crit}(g)=: Y$, and $f^{(2)}=g^{(2)}=0$, so that $\left(X^{(2)}, f^{(2)}\right)=\left(Y^{(2)}, g^{(2)}\right)$. However, as in Example 5.5 below, $\mathcal{P} \mathcal{V}_{U, f}^{\bullet} \neq \mathcal{P} \mathcal{V}_{V, g}^{\bullet}$. Thus, globally, $\mathcal{P} \mathcal{V}_{U, f}^{\bullet}$ is not determined up to isomorphism by $\left(X^{(2)}, f^{(2)}\right)$.

(c) Suppose $U$ is a complex manifold and $f: U \rightarrow \mathbb{C}$ is holomorphic, with Crit $(f)$ a single (not necessarily reduced) point $x$. The Mather-Yau Theorem [38. shows that the germ of $(U, f)$ at $x$ is determined up to non-canonical isomorphism by the complex analytic subspace $f^{(1)}=0$ in $X^{(1)}$, and hence by the pair $\left(X^{(1)}, f^{(1)}\right)$. Therefore, for isolated singularities, $\mathcal{P} \mathcal{V}_{U, f}^{\bullet}$ is determined up to non-canonical isomorphism by $\left(X^{(1)}, f^{(1)}\right)$. 
(d) Define $f: U \rightarrow \mathbb{C}$ by $U=\mathbb{C}$ and $f(z)=c z^{n}$ for $0 \neq c \in \mathbb{C}$ and $n>2$. This has an isolated singularity at 0 , and $\left(X^{(1)}, f^{(1)}\right)$ is independent of $c$. By moving $c$ in a circle round zero, we see that in this example $\mathcal{P} \mathcal{V}_{U, f}^{\bullet}$ is determined up to a $\mathbb{Z} / n \mathbb{Z}$ group of automorphisms. So the non-canonical isomorphisms of $\mathcal{P} \mathcal{V}_{U, f}^{\bullet}$ are not unique up to sign, in contrast to (a).

(e) Parts (a)-(d) leave open the question of whether $\mathcal{P} \mathcal{V}_{U, f}^{\bullet}$ is determined locally up to non-canonical isomorphism by $\left(X^{(1)}, f^{(1)}\right)$ for non-isolated singularities. We do not have a counterexample to this.

However, Gaffney and Hauser [19, §4] give examples of complex manifolds $U$ and holomorphic $f: U \rightarrow \mathbb{C}$ with $X=\operatorname{Crit}(f)$ non-isolated, such that the germ of $(U, f)$ at $x \in X$ is not determined up to non-canonical isomorphism by the germ of $\left(X^{(1)}, f^{(1)}\right)$ at $x$, in contrast to the Mather-Yau Theorem, and continuous families of distinct germs $[U, f, x]$ can have the same germ $\left[X^{(1)}, f^{(1)}, x\right]$. It seems likely that in examples of this kind, the mixed Hodge module $\mathcal{H V}_{U, f}^{\bullet}$ (which contains continuous Hodge-theoretic information) is not locally determined up to non-canonical isomorphism by $\left(X^{(1)}, f^{(1)}\right)$.

(f) For the example in (d), $\mathcal{P} \mathcal{V}_{U, f}^{\bullet}$ depends on $n=3,4, \ldots$, but $\left(X^{\text {red }}, f^{\text {red }}\right)=$ $(\{0\}, 0)$ is independent of $n$. So $\mathcal{P} \mathcal{V}_{U, f}^{\bullet}$ is not determined even locally up to non-canonical isomorphism by $\left(X^{\mathrm{red}}, f^{\mathrm{red}}\right)$.

\subsection{Proof of Proposition 4.3}

The $\mathbb{C}$-subscheme $X^{(k+1)}$ in $U$ is the zeroes of the ideal $I_{X}^{k+1} \subset \mathcal{O}_{U}$, which vanishes to order $k+1 \geqslant 2$ at $x \in X \subseteq X^{(k+1)} \subseteq U$. Hence $T_{x} X^{(k+1)}=T_{x} U$. As $\Phi: X^{(k+1)} \rightarrow Y^{(k+1)}$ is an isomorphism, it follows that

$$
T_{x} U=T_{x} X^{(k+1)} \cong T_{\Phi(x)} Y^{(k+1)}=T_{\Phi(x)} V .
$$

Therefore $n:=\operatorname{dim} U=\operatorname{dim} V$.

Choose a Zariski open neighbourhood $V^{\prime}$ of $\Phi(x)$ in $V$ and étale coordinates $\left(y_{1}, \ldots, y_{n}\right): V^{\prime} \rightarrow \mathbb{C}^{n}$ on $V^{\prime}$. Write $g^{\prime}=\left.g\right|_{V^{\prime}}$ and $Y^{\prime}=\operatorname{Crit}\left(g^{\prime}\right)=Y \cap V^{\prime}$, so that $Y^{\prime(k+1)}=Y^{(k+1)} \cap V^{\prime}$. Then $y_{a} \circ \Phi$ are regular functions on the open neighbourhood $\Phi^{-1}\left(V^{\prime}\right) \subseteq X^{(k+1)}$ of $x$ in $X^{(k+1)}$, so they extend Zariski locally from $X^{(k+1)}$ to $U$. Thus we can choose a Zariski open neighbourhood $U^{\prime}$ of $x$ in $U$ with $\Phi\left(X^{(k+1)} \cap U^{\prime}\right) \subseteq Y^{(k+1)} \cap V^{\prime}$, and regular functions $x_{i}: U^{\prime} \rightarrow \mathbb{C}$ with $\left.x_{i}\right|_{X^{(k+1)} \cap U^{\prime}}=\left.y_{i} \circ \Phi\right|_{X^{(k+1)} \cap U^{\prime}}$ for $i=1, \ldots, n$.

Write $f^{\prime}=\left.f\right|_{U^{\prime}}$ and $X^{\prime}=\operatorname{Crit}\left(f^{\prime}\right)=X \cap U^{\prime}$, so that $X^{\prime(k+1)}=X^{(k+1)} \cap X^{\prime}$. Since $\left(y_{1}, \ldots, y_{n}\right)$ are étale coordinates, $\left.\mathrm{d} y_{1}\right|_{\Phi(x)}, \ldots,\left.\mathrm{d} y_{n}\right|_{\Phi(x)}$ are a basis for $T_{\Phi(x)}^{*} V$, so $\left.\mathrm{d} x_{1}\right|_{x}, \ldots,\left.\mathrm{d} x_{n}\right|_{x}$ are a basis for $T_{x}^{*} X$ by (4.7). Hence by making $U^{\prime}$ smaller, we can suppose $\left(x_{1}, \ldots, x_{n}\right)$ are étale coordinates on $U^{\prime}$.

Consider the $\mathbb{C}$-scheme $U^{\prime} \times V^{\prime}$, with projections $\pi_{U^{\prime}}: U^{\prime} \times V^{\prime} \rightarrow U^{\prime}$, $\pi_{V^{\prime}}: U^{\prime} \times V^{\prime} \rightarrow V^{\prime}$, and write $x_{i}^{\prime}=x_{i} \circ \pi_{U^{\prime}}: U^{\prime} \times V^{\prime} \rightarrow \mathbb{C}, y_{i}^{\prime}=y_{i} \circ \pi_{V^{\prime}}:$ $U^{\prime} \times V^{\prime} \rightarrow \mathbb{C}$, so that $\left(x_{1}^{\prime}, \ldots, x_{n}^{\prime}, y_{1}^{\prime}, \ldots, y_{n}^{\prime}\right)$ are étale coordinates on $U^{\prime} \times V^{\prime}$. We have a morphism id $\times\left.\Phi\right|_{X^{\prime(k+1)}}: X^{(k+1)} \rightarrow U^{\prime} \times V^{\prime}$ which embeds $X^{\prime(k+1)}$ as a closed $\mathbb{C}$-subscheme of $U^{\prime} \times V^{\prime}$. The image $\left(\mathrm{id} \times\left.\Phi\right|_{X^{\prime(k+1)}}\right)\left(X^{\prime(k+1)}\right)$ is locally 
the zeroes of the sheaf of ideals

$$
\left(x_{i}^{\prime}-y_{i}^{\prime}, i=1, \ldots, n\right)+\pi_{U^{\prime}}^{-1}\left(I_{X}^{k+1}\right) \subset \mathcal{O}_{U^{\prime} \times V^{\prime}},
$$

where $\left(x_{i}^{\prime}-y_{i}^{\prime}, i=1, \ldots, n\right)$ denotes the ideal generated by $x_{i}^{\prime}-y_{i}^{\prime}: U^{\prime} \times V^{\prime} \rightarrow \mathbb{C}$ for $i=1, \ldots, n$, and $\pi_{U^{\prime}}^{-1}\left(I_{X}^{k+1}\right) \subset \mathcal{O}_{U^{\prime} \times V^{\prime}}$ the preimage ideal of $\left.I_{X}^{k+1}\right|_{U^{\prime}} \subset \mathcal{O}_{U^{\prime}}$.

Now $\left.\left(f \circ \pi_{U^{\prime}}-g \circ \pi_{V^{\prime}}\right)\right|_{(\mathrm{id} \times \Phi)\left(X^{\prime}(k+1)\right)}=0$ as $f^{(k+1)}=g^{(k+1)} \circ \Phi$. Hence

$$
f \circ \pi_{U^{\prime}}-g \circ \pi_{V^{\prime}} \in\left(x_{i}^{\prime}-y_{i}^{\prime}, i=1, \ldots, n\right)+\pi_{U^{\prime}}^{-1}\left(I_{X}^{k+1}\right) .
$$

Lifting (4.8) from $\left(x_{i}^{\prime}-y_{i}^{\prime}, i=1, \ldots, n\right)$ to $\left(x_{i}^{\prime}-y_{i}^{\prime}, i=1, \ldots, n\right)^{2}$, after making $U^{\prime}, V^{\prime}$ smaller if necessary, we can choose regular functions $A_{i}: U^{\prime} \times V^{\prime} \rightarrow \mathbb{C}$ for $i=1, \ldots, n$ such that

$$
f \circ \pi_{U^{\prime}}-g \circ \pi_{V^{\prime}}-\sum_{i=1}^{n} A_{i} \cdot\left(x_{i}^{\prime}-y_{i}^{\prime}\right) \in\left(x_{i}^{\prime}-y_{i}^{\prime}, i=1, \ldots, n\right)^{2}+\pi_{U^{\prime}}^{-1}\left(I_{X}^{k+1}\right) .
$$

Apply $\frac{\partial}{\partial x_{i}^{\prime}}$ to (4.9), using the étale coordinates $\left(x_{1}^{\prime}, \ldots, x_{n}^{\prime}, y_{1}^{\prime}, \ldots, y_{n}^{\prime}\right)$ on $U^{\prime} \times V^{\prime}$. Since $\frac{\partial}{\partial x_{i}^{\prime}}\left(f \circ \pi_{U^{\prime}}\right)=\frac{\partial f}{\partial x_{i}} \circ \pi_{U^{\prime}}$ and $\frac{\partial}{\partial x_{i}^{\prime}}\left(g \circ \pi_{V^{\prime}}\right)=0$, this gives

$$
\frac{\partial f}{\partial x_{i}} \circ \pi_{U^{\prime}}-A_{i} \in\left(x_{i}^{\prime}-y_{i}^{\prime}, i=1, \ldots, n\right)+\pi_{U^{\prime}}^{-1}\left(I_{X}^{k}\right) .
$$

Changing $A_{i}$ by an element of $\left(x_{i}^{\prime}-y_{i}^{\prime}, i=1, \ldots, n\right)$ can be absorbed in the ideal $\left(x_{i}^{\prime}-y_{i}^{\prime}, i=1, \ldots, n\right)^{2}$ in (4.9), so we can suppose $\frac{\partial f}{\partial x_{i}} \circ \pi_{U^{\prime}}-A_{i} \in \pi_{U^{\prime}}^{-1}\left(I_{X}^{k}\right)$. As $I_{X}=\left(\frac{\partial f}{\partial x_{j}}, j=1, \ldots, n\right)$, after making $U^{\prime}, V^{\prime}$ smaller we may write

$$
A_{i}=\frac{\partial f}{\partial x_{i}} \circ \pi_{U^{\prime}}+\sum_{j=1}^{n} B_{i j} \cdot \frac{\partial f}{\partial x_{j}} \circ \pi_{U^{\prime}}
$$

with $B_{i j} \in \pi_{U^{\prime}}^{-1}\left(I_{X}^{k-1}\right)$ for $i, j=1, \ldots, n$. Consider the matrix of functions $\left(\delta_{i j}+B_{i j}\right)_{i, j=1}^{n}$ on $U^{\prime} \times V^{\prime}$. At the point $(x, \Phi(x))$ in $U^{\prime} \times V^{\prime}$ this matrix is the identity, since $B_{i j}(x, \Phi(x))=0$ as $B_{i j} \in \pi_{U^{\prime}}^{-1}\left(I_{X}^{k-1}\right)$ with $k \geqslant 2$, so $\left(\delta_{i j}+B_{i j}\right)_{i, j=1}^{n}$ is invertible near $(x, \Phi(x))$, and making $U^{\prime}, V^{\prime}$ smaller we can suppose $\left(\delta_{i j}+B_{i j}\right)_{i, j=1}^{n}$ is invertible on $U^{\prime} \times V^{\prime}$. But in matrix notation we have

$$
\left(A_{i}\right)_{i=1}^{n}=\left(\delta_{i j}+B_{i j}\right)_{i, j=1}^{n}\left(\frac{\partial f}{\partial x_{j}} \circ \pi_{U^{\prime}}\right)_{j=1}^{n} .
$$

Hence in ideals in $\mathcal{O}_{U^{\prime} \times V^{\prime}}$ we have

$$
\left(A_{i}, i=1, \ldots, n\right)=\left(\frac{\partial f}{\partial x_{j}} \circ \pi_{U^{\prime}}, j=1, \ldots, n\right)=\pi_{U^{\prime}}^{-1}\left(I_{X}\right) \subset \mathcal{O}_{U^{\prime} \times V^{\prime}} .
$$

Now by (4.9), after making $U^{\prime}, V^{\prime}$ smaller if necessary, we may write

$$
\begin{aligned}
f \circ \pi_{U^{\prime}}- & g \circ \pi_{V^{\prime}}=\sum_{i=1}^{n} A_{i} \cdot\left(x_{i}^{\prime}-y_{i}^{\prime}\right) \\
& +\sum_{i, j=1}^{n} C_{i j} \cdot\left(x_{i}^{\prime}-y_{i}^{\prime}\right)\left(x_{j}^{\prime}-y_{j}^{\prime}\right)+\sum_{i, j=1}^{n} D_{i j} \cdot A_{i} A_{j},
\end{aligned}
$$


for regular functions $C_{i j}, D_{i j}: U^{\prime} \times V^{\prime} \rightarrow \mathbb{C}$ with $D_{i j} \in H^{0}\left(\pi_{U^{\prime}}^{-1}\left(I_{X}^{k-1}\right)\right)$ for $i, j=1, \ldots, n$, where in the last term we have used (4.12) to write two factors of $\pi_{U^{\prime}}^{-1}\left(I_{X}\right)$ in terms of $A_{1}, \ldots, A_{n}$.

Write $\left(z_{i j}\right)_{i, j=1}^{n}$ for the coordinates on $\mathbb{C}^{n^{2}}$. Let $W$ be a Zariski open neighbourhood of $\left(x, \Phi(x),(0)_{i, j=1}^{n}\right)$ in $U^{\prime} \times V^{\prime} \times \mathbb{C}^{n^{2}}$ to be chosen shortly, and let $T$ be the $\mathbb{C}$-subscheme of $W$ defined by

$$
\begin{aligned}
T=\{ & \left(u, v,\left(z_{i j}\right)_{i, j=1}^{n}\right) \in W \subseteq U^{\prime} \times V^{\prime} \times \mathbb{C}^{n^{2}}: \\
& x_{i}(u)-y_{i}(v)=\sum_{j=1}^{n} z_{i j} \cdot A_{j}(u, v), \quad i=1, \ldots, n, \\
& \left.z_{i j}+\sum_{l, m=1}^{n} C_{l m}(u, v) \cdot z_{l i} z_{m j}+D_{i j}(u, v)=0, \quad i, j=1, \ldots, n\right\} .
\end{aligned}
$$

Define $\mathbb{C}$-scheme morphisms $\pi_{U}: T \rightarrow U$ by $\pi_{U}:\left(u, v,\left(z_{i j}\right)_{i, j=1}^{n}\right) \mapsto u$ and $\pi_{V}: T \rightarrow V$ by $\pi_{V}:\left(u, v,\left(z_{i j}\right)_{i, j=1}^{n}\right) \mapsto v$.

Now $W \subseteq U^{\prime} \times V^{\prime} \times \mathbb{C}^{n^{2}}$ is smooth of dimension $n+n+n^{2}$, and in (4.14) we impose $n+n^{2}$ equations, so the expected dimension of $T$ is $\left(2 n+n^{2}\right)-\left(n+n^{2}\right)=$ $n$. The linearizations of the $n+n^{2}$ equations in (4.14) at $\left(u, v,\left(z_{i j}\right)_{i, j=1}^{n}\right)=$ $\left(x, \Phi(x),(0)_{i, j=1}^{n}\right)$ are

$$
\begin{array}{r}
\left.\mathrm{d} x_{i}\right|_{x}(\delta u)-\left.\mathrm{d} y_{i}\right|_{\Phi(x)}(\delta v)=0, \quad i=1, \ldots, n, \\
\delta z_{i j}+\left.\mathrm{d} D_{i j}\right|_{(x, \Phi(x))}(\delta u \oplus \delta v)=0, \quad i, j=1, \ldots, n,
\end{array}
$$

for $\delta u \in T_{x} U^{\prime}, \delta v \in T_{\Phi(x)} V^{\prime}$, and $\left(\delta z_{i j}\right)_{i, j=1}^{n} \in T_{(0)_{i, j=1}^{n}} \mathbb{C}^{n^{2}}$. As $\left.\mathrm{d} x_{1}\right|_{x}, \ldots,\left.\mathrm{d} x_{n}\right|_{x}$ are a basis for $T_{x}^{*} U^{\prime}$, the equations (4.15) are transverse, so that $T$ is smooth of dimension $n$ near $\left(x, \Phi(x),(0)_{i, j=1}^{n}\right)$.

The vector space of solutions $\left(\delta u, \delta v,\left(\delta z_{i j}\right)_{i, j=1}^{n}\right)$ to (4.15) is $T_{(x, \Phi(x),(0))} T$, where $\left.\mathrm{d} \pi_{U}\right|_{(x, \Phi(x),(0))}: T_{(x, \Phi(x),(0))} T \rightarrow T_{x} U$ maps $\left(\delta u, \delta v,\left(\delta z_{i j}\right)_{i, j=1}^{n}\right) \mapsto \delta u$, and $\left.\mathrm{d} \pi_{V}\right|_{(x, \Phi(x),(0))}: T_{(x, \Phi(x),(0))} T \rightarrow T_{\Phi(x)} V$ maps $\left(\delta u, \delta v,\left(\delta z_{i j}\right)_{i, j=1}^{n}\right) \mapsto \delta v$. Clearly, $\left.\mathrm{d} \pi_{U}\right|_{(x, \Phi(x),(0))},\left.\mathrm{d} \pi_{V}\right|_{(x, \Phi(x),(0))}$ are isomorphisms, so as $T$ is smooth near $\left(x, \Phi(x),(0)_{i, j=1}^{n}\right)$ and $U, V$ are smooth, we see that $\pi_{U}, \pi_{V}$ are étale near $(x, \Phi(x),(0))$. Thus, by choosing the open neighbourhood $(x, \Phi(x),(0)) \in W \subseteq$ $U^{\prime} \times V^{\prime} \times \mathbb{C}^{n^{2}}$ sufficiently small, we can suppose that $T$ is smooth of dimension $n$ and $\pi_{U}: T \rightarrow U, \pi_{V}: T \rightarrow V$ are étale. 
It remains to prove Proposition 4.3(a)-(c). For (a), we have

$$
\begin{aligned}
\left(f \circ \pi_{U}-g \circ \pi_{V}\right)\left(u, v,\left(z_{i j}\right)_{i, j=1}^{n}\right)=f(u)-g(v)=\left(f \circ \pi_{U^{\prime}}-g \circ \pi_{V^{\prime}}\right)(u, v) \\
=\sum_{i=1}^{n} A_{i}(u, v) \cdot\left(x_{i}(u)-y_{i}(v)\right)+\sum_{i, j=1}^{n} C_{i j}(u, v) \cdot\left(x_{i}(u)-y_{i}(v)\right) \\
\quad+\sum_{i, j=1}^{n} D_{i j}(u, v) \cdot A_{i}(u, v) A_{j}(u, v) \\
=\sum_{i=1}^{n} A_{i}(u, v) \cdot\left(\sum_{j=1}^{n} z_{i j} \cdot A_{j}(u, v)\right)+\sum_{i, j=1}^{n} C_{i j}(u, v) \cdot\left(\sum_{l=1}^{n} z_{i l} \cdot A_{l}(u, v)\right) \\
\quad+\sum_{i, j=1}^{n} D_{i j}(u, v) \cdot A_{i}(u, v) A_{j}(u, v) \\
=\sum_{i, j=1}^{n} A_{i}(u, v) A_{j}(u, v)\left[z_{i j}+\sum_{l, m=1}^{n}(u, v)\right)
\end{aligned}
$$

using (4.13) in the third step, the first equation of (4.14) in the fourth, rearranging and exchanging labels $i, l$ and $j, m$ in the fifth, and the second equation of (4.14) in the sixth. Hence $f \circ \pi_{U}-g \circ \pi_{V}=0: T \rightarrow \mathbb{C}$, proving (a).

For (b), using the morphism id $\times\left.\Phi\right|_{X} \times(0): X \rightarrow U \times V \times \mathbb{C}^{n^{2}} \supseteq W$, define $\tilde{X}=\left(\operatorname{id} \times\left.\Phi\right|_{X} \times(0)\right)^{-1}(W)$, so that $\tilde{X}$ is a Zariski open neighbourhood of $x$ in $X$. Then $\left(\mathrm{id} \times\left.\Phi\right|_{X} \times(0)\right)(\tilde{X})$ is a closed $\mathbb{C}$-subscheme of $W$. We claim that:

(i) $\left(\right.$ id $\left.\times\left.\Phi\right|_{X} \times(0)\right)(\tilde{X})$ is a closed $\mathbb{C}$-subscheme of $T \subseteq W$; and

(ii) $\left(\right.$ id $\left.\times\left.\Phi\right|_{X} \times(0)\right)(\tilde{X})$ is open and closed in $Q:=\operatorname{Crit}(e) \subseteq T$,

where $e:=f \circ \pi_{U}=g \circ \pi_{V}: T \rightarrow \mathbb{C}$. To prove (i), we have to show that the equations of (4.14) hold on $\left(\mathrm{id} \times\left.\Phi\right|_{X} \times(0)\right)(\tilde{X})$, which is true as $\left.x_{i}\right|_{\tilde{X}}=\left.y_{i} \circ \Phi\right|_{\tilde{X}}$, and $z_{i j} \circ(0)=0$, and $D_{i j} \circ\left(\mathrm{id} \times\left.\Phi\right|_{\tilde{X}}\right)=0$ as $D_{i j} \in H^{0}\left(\pi_{U^{\prime}}^{-1}\left(I_{X}^{k-1}\right)\right)$ for $k \geqslant 2$.

For (ii), as $\pi_{U}: T \rightarrow U$ is étale with $e=f \circ \pi_{U}$, we see that $\left.\pi_{U}\right|_{Q}: Q \rightarrow X$ is étale. But $\left.\left.\pi_{U}\right|_{Q} \circ\left(\mathrm{id} \times\left.\Phi\right|_{X} \times(0)\right)\right|_{\tilde{X}}=\mathrm{id}_{\tilde{X}}$. Hence $\left(\mathrm{id} \times\left.\Phi\right|_{X} \times(0)\right)(\tilde{X})$ is open in $Q$, and is also closed in $Q$ as it is closed in $T$. Thus, by making $W, T$ smaller to delete other components of $Q$, we can suppose that $Q=\left(\mathrm{id} \times\left.\Phi\right|_{X} \times(0)\right)(\tilde{X})$. Then $\left.\pi_{U}\right|_{Q}: Q \rightarrow \tilde{X}$ is an isomorphism with the Zariski open neighbourhood $\tilde{X}$ of $x$ in $X$. Since $\pi_{U}: T \rightarrow U$ is étale with $e=f \circ \pi_{U}$, this extends to the $k^{\text {th }}$ order thickenings, so $\left.\pi_{U}\right|_{Q^{(k)}}: Q^{(k)} \rightarrow \tilde{X}^{(k)}$ is an isomorphism, proving (b).

For (c), first note that $Q=\left(\mathrm{id} \times\left.\Phi\right|_{X} \times(0)\right)(\tilde{X})$, so $\left.\Phi \circ \pi_{U}\right|_{Q}=\left.\pi_{V}\right|_{Q}$ is immediate. We have to extend this to the thickening $Q^{(k)}$. Write $I_{Q} \subset \mathcal{O}_{T}$ for the ideal of functions vanishing on $Q$. Then $I_{Q}=\pi_{U}^{-1}\left(I_{X}\right)$ as $\pi_{U}$ identifies $Q$ with $\tilde{X} \subseteq X$. We have

$$
A_{i} \circ \pi_{U^{\prime} \times V^{\prime}} \in I_{Q} \quad \text { and } \quad D_{i j} \circ \pi_{U^{\prime} \times V^{\prime}} \in I_{Q}^{k-1},
$$

as $A_{i} \in H^{0}\left(\pi_{U^{\prime}}^{-1}\left(I_{X}\right)\right), D_{i j} \in H^{0}\left(\pi_{U^{\prime}}^{-1}\left(I_{X}^{k-1}\right)\right)$. The second equation of (4.14) then shows that

$$
z_{i j} \circ \pi_{\mathbb{C}^{n^{2}}} \in I_{Q}^{k-1}
$$


since $Q=\left(\mathrm{id} \times\left.\Phi\right|_{X} \times(0)\right)(\tilde{X})$ implies that $z_{i j} \circ \pi_{\mathbb{C}^{n^{2}}}=0$ on $Q$, so we can neglect the terms $\sum_{l, m=1}^{n} C_{l m}(u, v) \cdot z_{l i} z_{m j}$. Hence the first equation of (4.14) gives

$$
x_{i} \circ \pi_{U}-y_{i} \circ \pi_{V} \in I_{Q}^{k} .
$$

As $I_{Q}^{k}$ vanishes on $Q^{(k)}$, and $\left.x_{i}\right|_{X^{\prime}}=\left.y_{i} \circ \Phi\right|_{X^{\prime}}$, this gives

$$
y_{i} \circ\left(\left.\Phi \circ \pi_{U}\right|_{Q^{(k)}}\right)=\left.x_{i} \circ \pi_{U}\right|_{Q^{(k)}}=y_{i} \circ\left(\left.\pi_{V}\right|_{Q^{(k)}}\right) .
$$

Thus $\left.\Phi \circ \pi_{U}\right|_{Q^{(k)}}=\left.\pi_{V}\right|_{Q^{(k)}}$ follows, as $\left(y_{1}, \ldots, y_{n}\right)$ are étale coordinates on $V$ near $\pi_{V}(Q)$ and $\left.\Phi \circ \pi_{U}\right|_{Q}=\left.\pi_{V}\right|_{Q}$. This proves (c), and Proposition 4.3.

\subsection{Proof of Theorem 4.2 for $\mathbb{C}$-schemes}

Let $U, V, f, g, X, Y$ and $\Phi: X^{(3)} \rightarrow Y^{(3)}$ be as in Theorem 4.2. Pick $x \in X$, and apply Proposition 4.3 with $k=2$. This gives a smooth $\mathbb{C}$-scheme $T$ and étale morphisms $\pi_{U}: T \rightarrow U, \pi_{V}: T \rightarrow V$ with $e:=f \circ \pi_{U}=g \circ \pi_{V}: T \rightarrow \mathbb{C}$ and $Q:=\operatorname{Crit}(e)$, such that $\left.\pi_{U}\right|_{Q^{(2)}}: Q^{(2)} \rightarrow X^{(2)}$ is an étale open neighbourhood of $x$ in $X^{(2)}$, and $\left.\Phi \circ \pi_{U}\right|_{Q^{(2)}}=\left.\pi_{V}\right|_{Q^{(2)}}: Q^{(2)} \rightarrow Y^{(2)}$. Actually Proposition 4.3 proves more, that $\left.\pi_{U}\right|_{Q^{(2)}}: Q^{(2)} \rightarrow X^{(2)}$ is an isomorphism with a Zariski open set $x \in \tilde{X}^{(2)} \subseteq X^{(2)}$, but we will not use this.

Thus, we can choose $\left\{\left(T^{a}, \pi_{U}^{a}, \pi_{V}^{a}, e^{a}, Q^{a}\right): a \in A\right\}$, where $A$ is an indexing set, such that $T^{a}, \pi_{U}^{a}, \pi_{V}^{a}, e^{a}, Q^{a}$ satisfy the conditions above for each $a \in A$, and $\left\{\left.\pi_{U}^{a}\right|_{Q^{a}}: Q^{a} \rightarrow X\right\}_{a \in A}$ is an étale open cover of $X$. Then for each $a \in A$, by Definition 2.15 we have isomorphisms

$$
\mathcal{P} \mathcal{V}_{\pi_{U}^{a}}:\left.\mathcal{P} \mathcal{V}_{T^{a}, e^{a}}^{\bullet} \longrightarrow \pi_{U}^{a}\right|_{Q^{a}} ^{*}\left(\mathcal{P} \mathcal{V}_{U, f}^{\bullet}\right), \quad \mathcal{P} \mathcal{V}_{\pi_{V}^{a}}:\left.\mathcal{P} \mathcal{V}_{T^{a}, e^{a}}^{\bullet} \longrightarrow \pi_{V}^{a}\right|_{Q^{a}} ^{*}\left(\mathcal{P} \mathcal{V}_{V, f}^{\bullet}\right)
$$

Noting that $\left.\pi_{V}^{a}\right|_{Q^{a}}=\left.\left.\Phi\right|_{X} \circ \pi_{U}^{a}\right|_{Q^{a}}$, we may define an isomorphism

$$
\Omega^{a}=\mathcal{P} \mathcal{V}_{\pi_{V}^{a}} \circ \mathcal{P} \mathcal{V}_{\pi_{U}^{a}}^{-1}:\left.\left.\pi_{U}^{a}\right|_{Q^{a}} ^{*}\left(\mathcal{P} \mathcal{V}_{U, f}^{\bullet}\right) \longrightarrow \pi_{U}^{a}\right|_{Q^{a}} ^{*}\left(\left.\Phi\right|_{X} ^{*}\left(\mathcal{P} \mathcal{V}_{V, f}^{\bullet}\right)\right)
$$

For $a, b \in A$, define $T^{a b}=T^{a} \times_{\pi_{U}^{a}, U, \pi_{U}^{b}} T^{b}$ to be the $\mathbb{C}$-scheme fibre product, so that $T^{a b}$ is a smooth $\mathbb{C}$-scheme and the projections $\Pi_{T^{a}}: T^{a b} \rightarrow T^{a}, \Pi_{T^{b}}$ : $T^{a b} \rightarrow T^{b}$ are étale. Define $e^{a b}=e^{a} \circ \Pi_{T^{a}}: T^{a} \rightarrow \mathbb{C}$. Then

$$
\begin{aligned}
e^{a b} & =e^{a} \circ \Pi_{T^{a}}=g \circ \pi_{V}^{a} \circ \Pi_{T^{a}}=f \circ \pi_{U}^{a} \circ \Pi_{T^{a}} \\
& =f \circ \pi_{U}^{b} \circ \Pi_{T^{b}}=g \circ \pi_{V}^{b} \circ \Pi_{T^{b}}=e^{b} \circ \Pi_{T^{b}} .
\end{aligned}
$$

Write $Q^{a b}=\operatorname{Crit}\left(e^{a b}\right)$. Then $\left.\Pi_{T^{a}}\right|_{Q^{a b}}: Q^{a b} \rightarrow Q^{a}$ and $\left.\Pi_{T^{b}}\right|_{Q^{a b}}: Q^{a b} \rightarrow Q^{b}$ are étale. Now $\pi_{U}^{a} \circ \Pi_{T^{a}}=\pi_{U}^{b} \circ \Pi_{T^{b}}$ and $\left.\Phi \circ \pi_{U}^{a}\right|_{Q^{a(2)}}=\left.\pi_{V}^{a}\right|_{Q^{a(2)}}$ imply that

$$
\begin{aligned}
\left.\left(\pi_{V}^{a} \circ \Pi_{T^{a}}\right)\right|_{Q^{a b(2)}}=\left.\left.\pi_{V}^{a}\right|_{Q^{a(2)}} \circ \Pi_{T^{a}}\right|_{Q^{a b(2)}} & \\
& =\left.\left.\left.\Phi\right|_{X^{(2)}} \circ \pi_{U}^{a}\right|_{Q^{a(2)}} \circ \Pi_{T^{a}}\right|_{Q^{a b(2)}}=\left.\left.\Phi\right|_{X^{(2)}} \circ\left(\pi_{U}^{a} \circ \Pi_{T^{a}}\right)\right|_{Q^{a b(2)}} \\
& =\left.\left.\Phi\right|_{X^{(2)}} \circ\left(\pi_{U}^{b} \circ \Pi_{T^{b}}\right)\right|_{Q^{a b(2)}}=\left.\left.\left.\Phi\right|_{X^{(2)}} \circ \pi_{U}^{a}\right|_{Q^{b(2)}} \circ \Pi_{T^{b}}\right|_{Q^{a b(2)}} \\
& =\left.\left.\pi_{V}^{a}\right|_{Q^{b(2)}} \circ \Pi_{T^{b}}\right|_{Q^{a b(2)}}=\left.\left(\pi_{V}^{b} \circ \Pi_{T^{b}}\right)\right|_{Q^{a b(2)}} .
\end{aligned}
$$


Hence $\left.\left(\pi_{V}^{a} \circ \Pi_{T^{a}}\right)\right|_{Q^{a b}}=\left.\left(\pi_{V}^{b} \circ \Pi_{T^{b}}\right)\right|_{Q^{a b}}$. Moreover, as $\left.T Q^{a b(2)}\right|_{Q^{a b}}=\left.T\left(T^{a b}\right)\right|_{Q^{a b}}$, we see that $\left.\mathrm{d}\left(\pi_{V}^{a} \circ \Pi_{T^{a}}\right)\right|_{Q^{a b}}=\left.\mathrm{d}\left(\pi_{V}^{b} \circ \Pi_{T^{b}}\right)\right|_{Q^{a b}}$, so that

$$
\left.\left.\mathrm{d}\left(\pi_{V}^{b} \circ \Pi_{T^{b}}\right)\right|_{Q^{a b}} ^{-1} \circ \mathrm{d}\left(\pi_{V}^{a} \circ \Pi_{T^{a}}\right)\right|_{Q^{a b}}=\mathrm{id}:\left.\left.T\left(T^{a b}\right)\right|_{Q^{a b}} \longrightarrow T\left(T^{a b}\right)\right|_{Q^{a b}} .
$$

So $\operatorname{det}\left(\left.\left.\mathrm{d}\left(\pi_{V}^{b} \circ \Pi_{T^{b}}\right)\right|_{Q^{a b}} ^{-1} \circ \mathrm{d}\left(\pi_{V}^{a} \circ \Pi_{T^{a}}\right)\right|_{Q^{a b}}\right)=1$. Thus, applying Theorem 3.1 with $T^{a b}, V, Q^{a b}, \pi_{V}^{a} \circ \Pi_{T^{a}}, \pi_{V}^{b} \circ \Pi_{T^{b}}, e^{a b}, f$ in place of $V, W, X, \Phi, \Psi, f, g$ gives

$$
\mathcal{P} \mathcal{V}_{\pi_{V}^{a} \circ \Pi_{T^{a}}}=\mathcal{P} \mathcal{V}_{\pi_{V}^{b} \circ \Pi_{T^{b}}}:\left.\mathcal{P} \mathcal{V}_{T^{a b}, e^{a b}}^{\bullet} \longrightarrow\left(\pi_{V}^{a} \circ \Pi_{T^{a}}\right)\right|_{Q^{a b}} ^{*}\left(\mathcal{P} \mathcal{V}_{V, g}^{\bullet}\right)
$$

Now

$$
\begin{aligned}
\left.\Pi_{T^{a}}\right|_{Q^{a b}} ^{*} & \left(\Omega^{a}\right)=\left.\left.\Pi_{T^{a}}\right|_{Q^{a b}} ^{*}\left(\mathcal{P} \mathcal{V}_{\pi_{V}^{a}}\right) \circ \Pi_{T^{a}}\right|_{Q^{a b}} ^{*}\left(\mathcal{P} \mathcal{V}_{\pi_{U}^{a}}\right)^{-1} \\
& =\left[\left.\Pi_{T^{a}}\right|_{Q^{a b}} ^{*}\left(\mathcal{P} \mathcal{V}_{\pi_{V}^{a}}\right) \circ \mathcal{P} \mathcal{V}_{\Pi_{T^{a}}}\right] \circ\left[\left.\Pi_{T^{a}}\right|_{Q^{a b}} ^{*}\left(\mathcal{P} \mathcal{V}_{\pi_{U}^{a}}\right) \circ \mathcal{P} \mathcal{V}_{\Pi_{T^{a}}}\right]^{-1} \\
& =\mathcal{P} \mathcal{V}_{\pi_{V}^{a} \circ \Pi_{T^{a}}} \circ \mathcal{P} \mathcal{V}_{\pi_{U}^{a} \circ \Pi_{T^{a}}}^{-1}=\mathcal{P} \mathcal{V}_{\pi_{V}^{b} \circ \Pi_{T^{b}}} \circ \mathcal{P} \mathcal{V}_{\pi_{U}^{b} \circ \Pi_{T^{b}}}^{-1} \\
& =\left[\left.\Pi_{T^{b}}\right|_{Q^{a b}} ^{*}\left(\mathcal{P} \mathcal{V}_{\pi_{V}^{a}}\right) \circ \mathcal{P} \mathcal{V}_{\Pi_{T^{b}}}\right] \circ\left[\left.\Pi_{T^{b}}\right|_{Q^{a b}} ^{*}\left(\mathcal{P} \mathcal{V}_{\pi_{U}^{b}}\right) \circ \mathcal{P} \mathcal{V}_{\Pi_{T^{b}}}\right]^{-1} \\
& =\left.\left.\Pi_{T^{b}}\right|_{Q^{a b}} ^{*}\left(\mathcal{P} \mathcal{V}_{\pi_{V}^{b}}\right) \circ \Pi_{T^{b}}^{*}\right|_{Q^{a b}}\left(\mathcal{P} \mathcal{V}_{\pi_{U}^{b}}\right)^{-1}=\left.\Pi_{T^{b}}\right|_{Q^{a b}} ^{*}\left(\Omega^{b}\right),
\end{aligned}
$$

using (4.16) in the first and seventh steps, (2.18) in the third and fifth, and (4.19) and $\pi_{U}^{a} \circ \Pi_{T^{a}}=\pi_{U}^{b} \circ \Pi_{T^{b}}$ in the fourth. Therefore Theorem[2.7(i) applied to the étale open cover $\left\{\left.\pi_{U}^{a}\right|_{Q^{a}}: Q^{a} \rightarrow X\right\}_{a \in A}$ of $X$ shows that there is a unique isomorphism $\Omega_{\Phi}$ in (4.2) with $\left.\pi_{U}^{a}\right|_{Q^{a}} ^{*}\left(\Omega_{\Phi}\right)=\Omega^{a}$ for all $a \in A$.

Suppose $\left\{\left(T^{a}, \ldots, Q^{a}\right): a \in A\right\}$ and $\left\{\left(T^{\prime a}, \ldots, Q^{\prime a}\right): a \in A^{\prime}\right\}$ are alternative choices above, yielding morphisms $\Omega_{\Phi}$ and $\Omega_{\Phi}^{\prime}$ in (4.2). By running the same construction using the family $\left\{\left(T^{a}, \ldots, Q^{a}\right): a \in A\right\} \amalg\left\{\left(T^{\prime a}, \ldots, Q^{\prime a}\right): a \in A^{\prime}\right\}$, we get a third morphism $\Omega_{\Phi}^{\prime \prime}$ in (4.2), such that $\left.\pi_{U}^{a}\right|_{Q^{a}} ^{*}\left(\Omega_{\Phi}\right)=\Omega^{a}=\left.\pi_{U}^{a}\right|_{Q^{a}} ^{*}\left(\Omega_{\Phi}^{\prime \prime}\right)$ for $a \in A$, giving $\Omega_{\Phi}=\Omega_{\Phi}^{\prime \prime}$, and $\left.\pi_{U}^{\prime a}\right|_{Q^{\prime}} ^{*}\left(\Omega_{\Phi}^{\prime}\right)=\Omega^{\prime a}=\left.\pi_{U}^{\prime a}\right|_{Q^{\prime a}} ^{*}\left(\Omega_{\Phi}^{\prime \prime}\right)$ for $a \in A^{\prime}$, which forces $\Omega_{\Phi}^{\prime}=\Omega_{\Phi}^{\prime \prime}$. Thus $\Omega_{\Phi}=\Omega_{\Phi}^{\prime}$, so $\Omega_{\Phi}$ is independent of the choice of $\left\{\left(T^{a}, \ldots, Q^{a}\right): a \in A\right\}$ above.

Let $T, \pi_{U}, \pi_{V}, e, Q$ be as in Theorem 4.2 Applying the argument above using the family $\left\{\left(T^{a}, \ldots, Q^{a}\right): a \in A\right\} \amalg\left\{\left(T, \pi_{U}, \pi_{V}, e, Q\right)\right\}$ shows that $\Omega_{\Phi}$ satisfies $\left.\pi_{U}\right|_{Q} ^{*}\left(\Omega_{\Phi}\right)=\mathcal{P} \mathcal{V}_{\pi_{V}} \circ \mathcal{P} \mathcal{V}_{\pi_{U}}^{-1}$, by (4.16). Thus (4.3) holds.

To show that (4.4)-(4.5) commute, we can combine equations (2.16)-(2.17), (4.16) and $\left.\pi_{U}^{a}\right|_{Q^{a}} ^{*}\left(\Omega_{\Phi}\right)=\Omega^{a}$ to show that $\left.\pi_{U}^{a}\right|_{Q^{a}} ^{*}$ applied to (4.4)-(4.5) commute in $\operatorname{Perv}\left(Q^{a}\right)$ for each $a \in A$, so (4.4) -4.5) commute by Theorem 2.7(i).

Suppose there exists an étale morphism $\Xi: U \rightarrow V$ with $f=g \circ \Xi: U \rightarrow \mathbb{C}$ and $\left.\Xi\right|_{X^{(3)}}=\Phi: X^{(3)} \rightarrow Y^{(3)}$. Then as we have to prove, we have

$$
\mathcal{P} \mathcal{V}_{\Xi}=\mathrm{id}_{X}^{*}\left(\Omega_{\Phi}\right) \circ \mathcal{P} \mathcal{V}_{\mathrm{id}_{U}}=\Omega_{\Phi} \circ \mathrm{id}_{\mathcal{P} \mathcal{V}_{U, f}}=\Omega_{\Phi}
$$

where in the first step we use (4.3) with $T=U, \pi_{U}=\operatorname{id}_{U}, \pi_{V}=\Xi, e=f$, and $Q=X$, and in the second we use $\mathcal{P} \mathcal{V}_{\mathrm{id}_{U}}=\operatorname{id}_{\mathcal{P} \mathcal{V}_{U, f}^{\bullet}}$ from Definition 2.15.

Suppose $W$ is another smooth $\mathbb{C}$-scheme, $h: W \rightarrow \mathbb{C}$ is regular, $Z=\operatorname{Crit}(h)$, and $\Psi: Y^{(3)} \rightarrow Z^{(3)}$ is an isomorphism with $h^{(3)} \circ \Psi=g^{(3)}$. Let $x \in X$, and set 
$y=\Phi(x) \in Y$. Proposition 4.3 for $x, \Phi$ gives a smooth $T$ and étale $\pi_{U}: T \rightarrow U$, $\pi_{V}: T \rightarrow V$ with $e:=f \circ \pi_{U}=g \circ \pi_{V}$ and $Q:=\operatorname{Crit}(e)$, such that $\left.\pi_{U}\right|_{Q^{(2)}}:$ $Q^{(2)} \rightarrow X^{(2)}$ is an étale open neighbourhood of $x$, and $\left.\Phi \circ \pi_{U}\right|_{Q^{(2)}}=\left.\pi_{V}\right|_{Q^{(2)}}$. Proposition 4.3 for $y, \Psi$ gives smooth $\tilde{T}$ and étale $\tilde{\pi}_{V}: \tilde{T} \rightarrow V, \tilde{\pi}_{W}: \tilde{T} \rightarrow W$ with $\tilde{e}:=g \circ \tilde{\pi}_{V}=h \circ \tilde{\pi}_{W}$ and $\tilde{Q}:=\operatorname{Crit}(\tilde{e})$, such that $\tilde{\pi}_{V} \mid \tilde{Q}^{(2)}: \tilde{Q}^{(2)} \rightarrow Y^{(2)}$ is an étale open neighbourhood of $y$, and $\Psi \circ \tilde{\pi}_{V}\left|\tilde{Q}^{(2)}=\tilde{\pi}_{W}\right| \tilde{Q}^{(2)}$.

Define $\hat{T}=T \times_{\pi_{V}, V, \tilde{\pi}_{V}} \tilde{T}$ with projections $\Pi_{T}: \hat{T} \rightarrow T, \Pi_{\tilde{T}}: \hat{T} \rightarrow \tilde{T}$. Then $\hat{T}$ is smooth and $\Pi_{T}, \Pi_{\tilde{T}}$ are étale, as $T, \tilde{T}, V$ are smooth and $\pi_{V}, \tilde{\pi}_{V}$ étale. Define $\hat{\pi}_{U}=\pi_{U} \circ \Pi_{T}: \hat{T} \rightarrow U$ and $\hat{\pi}_{W}=\tilde{\pi}_{W} \circ \Pi_{\tilde{T}}: \hat{T} \rightarrow W$. Then $\hat{\pi}_{U}, \hat{\pi}_{W}$ are étale. Set $\hat{e}=f \circ \hat{\pi}_{U}: \hat{T} \rightarrow \mathbb{C}$, and write $\hat{Q}=\operatorname{Crit}(\hat{e})$. Then

$$
\hat{e}=f \circ \hat{\pi}_{U}=f \circ \pi_{U} \circ \Pi_{T}=g \circ \pi_{V} \circ \Pi_{T}=g \circ \tilde{\pi}_{V} \circ \Pi_{\tilde{T}}=h \circ \tilde{\pi}_{W} \circ \Pi_{\tilde{T}}=h \circ \hat{\pi}_{W} .
$$

Also $\left.\hat{\pi}_{U}\right|_{\hat{Q}^{(2)}}: \hat{Q}^{(2)} \rightarrow X^{(2)}$ is an étale open neighbourhood of $x$, and

$$
\begin{aligned}
\left.(\Psi \circ \Phi) \circ \hat{\pi}_{U}\right|_{\hat{Q}^{(2)}} & =\left.\left.\Psi \circ \Phi \circ \pi_{U}\right|_{Q^{(2)}} \circ \prod_{T}\left|\hat{Q}^{(2)}=\Psi \circ \pi_{V}\right|_{Q^{(2)}} \circ \Pi_{T}\right|_{\hat{Q}^{(2)}} \\
& =\Psi \circ \tilde{\pi}_{V}\left|\tilde{Q}^{(2)} \circ \Pi_{\tilde{T}}\right| \hat{Q}^{(2)}=\tilde{\pi}_{W}\left|\tilde{Q}^{(2)} \circ \Pi_{\tilde{T}}\right| \hat{Q}^{(2)}=\hat{\pi}_{W} \mid \hat{Q}^{(2)} .
\end{aligned}
$$

Thus we may apply (4.3) for $\Omega_{\Phi}$ with $T, \pi_{U}, \pi_{V}, \ldots, Q$, and for $\Omega_{\Psi}$ with $\tilde{T}, \tilde{\pi}_{V}, \tilde{\pi}_{W}, \ldots, \tilde{Q}$, and for $\Omega_{\Psi \circ \Phi}$ with $\hat{T}, \hat{\pi}_{U}, \hat{\pi}_{W}, \ldots, \hat{Q}$. This yields

$$
\begin{gathered}
\left.\pi_{U}\right|_{Q} ^{*}\left(\Omega_{\Phi}\right)=\mathcal{P} \mathcal{V}_{\pi_{V}} \circ \mathcal{P} \mathcal{V}_{\pi_{U}}^{-1},\left.\quad \tilde{\pi}_{V}\right|_{\tilde{Q}^{2}} ^{*}\left(\Omega_{\Psi}\right)=\mathcal{P} \mathcal{V}_{\tilde{\pi}_{W}} \circ \mathcal{P} \mathcal{V}_{\tilde{\pi}_{V}}^{-1}, \\
\left.\hat{\pi}_{U}\right|_{\hat{Q}} ^{*}\left(\Omega_{\Psi \circ \Phi}\right)=\mathcal{P} \mathcal{V}_{\hat{\pi}_{W}} \circ \mathcal{P} \mathcal{V}_{\hat{\pi}_{U}}^{-1} .
\end{gathered}
$$

Now

$$
\begin{aligned}
& \left.\hat{\pi}_{U}\right|_{\hat{Q}} ^{*}\left(\Omega_{\Psi \circ \Phi}\right)=\mathcal{P} \mathcal{V}_{\hat{\pi}_{W}} \circ \mathcal{P} \mathcal{V}_{\hat{\pi}_{U}}^{-1}=\mathcal{P} \mathcal{V}_{\tilde{\pi}_{W} \circ \Pi_{\tilde{T}}} \circ \mathcal{P} \mathcal{V}_{\pi_{U} \circ \Pi_{T}}^{-1} \\
& =\left[\left.\Pi_{\tilde{T}}\right|_{\hat{Q}} ^{*}\left(\mathcal{P} \mathcal{V}_{\tilde{\pi}_{W}}\right) \circ \mathcal{P} \mathcal{V}_{\Pi_{\tilde{T}}}\right] \circ\left[\left.\Pi_{T}\right|_{\hat{Q}} ^{*}\left(\mathcal{P} \mathcal{V}_{\pi_{U}}\right) \circ \mathcal{P} \mathcal{V}_{\Pi_{T}}\right]^{-1} \\
& =\left.\left.\left.\Pi_{\tilde{T}}\right|_{\hat{Q}} ^{*}\left(\mathcal{P} \mathcal{V}_{\tilde{\pi}_{W}} \circ \mathcal{P} \mathcal{V}_{\tilde{\pi}_{V}}^{-1}\right) \circ \Pi_{\tilde{T}}\right|_{\hat{Q}} ^{*}\left(\mathcal{P} \mathcal{V}_{\tilde{\pi}_{V}}\right) \circ \mathcal{P} \mathcal{V}_{\Pi_{\tilde{T}}} \circ \mathcal{P} \mathcal{V}_{\Pi_{T}}^{-1} \circ \Pi_{T}\right|_{\hat{Q}} ^{*}\left(\mathcal{P} \mathcal{V}_{\pi_{U}}^{-1}\right) \\
& =\left.\left.\Pi_{\tilde{T}}\right|_{\hat{Q}} ^{*}\left(\left.\tilde{\pi}_{V}\right|_{\tilde{Q}} ^{*}\left(\Omega_{\Psi}\right)\right) \circ \mathcal{P} \mathcal{V}_{\tilde{\pi}_{V} \circ \Pi_{\tilde{T}}} \circ \mathcal{P} \mathcal{V}_{\Pi_{T}}^{-1} \circ \Pi_{T}\right|_{\hat{Q}} ^{*}\left(\mathcal{P} \mathcal{V}_{\pi_{U}}^{-1}\right) \\
& =\left.\left[\left.\left.\tilde{\pi}_{V}\right|_{\tilde{Q}} \circ \Pi_{\tilde{T}}\right|_{\hat{Q}}\right]^{*}\left(\Omega_{\Psi}\right) \circ \mathcal{P} \mathcal{V}_{\pi_{V} \circ \Pi_{T}} \circ \mathcal{P} \mathcal{V}_{\Pi_{T}}^{-1} \circ \Pi_{T}\right|_{\hat{Q}} ^{*}\left(\mathcal{P} \mathcal{V}_{\pi_{U}}^{-1}\right) \\
& =\left.\left.\left[\left.\left.\pi_{V}\right|_{Q} \circ \Pi_{T}\right|_{\hat{Q}}\right]^{*}\left(\Omega_{\Psi}\right) \circ \Pi_{T}\right|_{\hat{Q}} ^{*}\left(\mathcal{P} \mathcal{V}_{\pi_{V}}\right) \circ \mathcal{P} \mathcal{V}_{\Pi_{T}} \circ \mathcal{P} \mathcal{V}_{\Pi_{T}}^{-1} \circ \Pi_{T}\right|_{\hat{Q}} ^{*}\left(\mathcal{P} \mathcal{V}_{\pi_{U}}^{-1}\right) \\
& =\left.\left[\left.\left.\left.\Phi\right|_{X} \circ \pi_{U}\right|_{Q} \circ \Pi_{T}\right|_{\hat{Q}}\right]^{*}\left(\Omega_{\Psi}\right) \circ \Pi_{T}\right|_{\hat{Q}} ^{*}\left(\mathcal{P} \mathcal{V}_{\pi_{V}} \circ \mathcal{P} \mathcal{V}_{\pi_{U}}^{-1}\right) \\
& =\left.\left.\left(\pi_{U} \circ \Pi_{T}\right)\right|_{\hat{Q}} ^{*}\left(\left.\Phi\right|_{X} ^{*}\left(\Omega_{\Psi}\right)\right) \circ \Pi_{T}\right|_{\hat{Q}} ^{*}\left(\left.\pi_{U}\right|_{Q} ^{*}\left(\Omega_{\Phi}\right)\right)=\left.\hat{\pi}_{U}\right|_{\hat{Q}} ^{*}\left(\left.\Phi\right|_{X} ^{*}\left(\Omega_{\Psi}\right) \circ \Omega_{\Phi}\right) \text {, }
\end{aligned}
$$

using (4.21) in the first, fifth and ninth steps, (2.18) in the third, fifth and seventh, $\pi_{V} \circ \Pi_{T}=\tilde{\pi}_{V} \circ \Pi_{\tilde{T}}$ in the sixth and seventh, and $\left.\Phi \circ \pi_{U}\right|_{Q}=\left.\pi_{V}\right|_{Q}$ in the eighth. Thus, for each $x \in X$, we have constructed an étale open neighbourhood $\left.\hat{\pi}_{U}\right|_{\hat{Q}}: \hat{Q} \rightarrow X$ such that $\left.\hat{\pi}_{U}\right|_{\hat{Q}} ^{*}$ applied to (4.6) holds. Equation (4.6) follows by Theorem 2.7(i). Finally, if $U=V, f=g, X=Y$ and $\Phi=\operatorname{id}_{X^{(3)}}$ then $\Omega_{\operatorname{id}_{X}(3)}=\operatorname{id}_{\mathcal{P} \mathcal{V}_{U, f}}$ follows by taking $\Xi=\operatorname{id}_{U}$ in the fourth paragraph of the theorem. This proves Theorem 4.2 for perverse sheaves on $\mathbb{C}$-schemes. 


\section{3 $\mathscr{D}$-modules and mixed Hodge modules}

Once again, the proof of Proposition 4.3 is completely algebraic, so applies in the other contexts of $2.6-2.10$. Theorem 4.2 then follows for our other contexts from that and the general framework of $\$ 2.5$.

\section{$5 \quad$ Stabilizing vanishing cycles}

To set up notation for our main result, which is Theorem 5.4 below, we need the following theorem, which is proved in Joyce [23, Prop.s 2.22, $2.23 \&$ 2.25].

Theorem 5.1 (Joyce [23]). Let $U, V$ be smooth $\mathbb{C}$-schemes, $f: U \rightarrow \mathbb{C}, g:$ $V \rightarrow \mathbb{C}$ be regular, and $X=\operatorname{Crit}(f), Y=\operatorname{Crit}(g)$ as $\mathbb{C}$-subschemes of $U, V$. Let $\Phi: U \hookrightarrow V$ be a closed embedding of $\mathbb{C}$-schemes with $f=g \circ \Phi: U \rightarrow \mathbb{C}$, and suppose $\left.\Phi\right|_{X}: X \rightarrow V \supseteq Y$ is an isomorphism $\left.\Phi\right|_{X}: X \rightarrow Y$. Then:

(i) For each $x \in X \subseteq U$ there exist smooth $\mathbb{C}$-schemes $U^{\prime}, V^{\prime}$, a point $x^{\prime} \in U^{\prime}$ and morphisms $\iota: U^{\prime} \rightarrow U, \jmath: V^{\prime} \rightarrow V, \Phi^{\prime}: U^{\prime} \rightarrow V^{\prime}, \alpha: V^{\prime} \rightarrow U$ and $\beta: V^{\prime} \rightarrow \mathbb{C}^{n}$, where $n=\operatorname{dim} V-\operatorname{dim} U$, such that $\iota\left(x^{\prime}\right)=x$, and $\iota, \jmath$ and $\alpha \times \beta: V \rightarrow U \times \mathbb{C}^{n}$ are étale, and the following diagram commutes

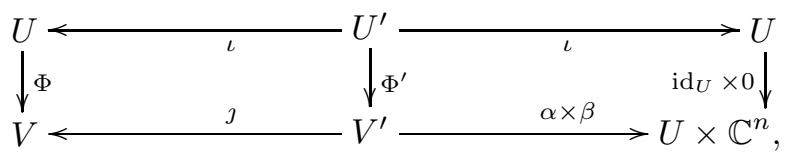

and $g \circ \jmath=f \circ \alpha+\left(z_{1}^{2}+\cdots+z_{n}^{2}\right) \circ \beta: V^{\prime} \rightarrow \mathbb{C}$. Thus, setting $f^{\prime}:=f \circ \iota: U^{\prime} \rightarrow \mathbb{C}$, $g^{\prime}:=g \circ \jmath: V^{\prime} \rightarrow \mathbb{C}, X^{\prime}:=\operatorname{Crit}\left(f^{\prime}\right) \subseteq U^{\prime}$, and $Y^{\prime}:=\operatorname{Crit}\left(g^{\prime}\right) \subseteq V^{\prime}$, then $f^{\prime}=g^{\prime} \circ \Phi^{\prime}: U^{\prime} \rightarrow \mathbb{C}$, and $\left.\Phi^{\prime}\right|_{X^{\prime}}: X^{\prime} \rightarrow Y^{\prime},\left.\iota\right|_{X^{\prime}}: X^{\prime} \rightarrow X,\left.\jmath\right|_{Y^{\prime}}: Y^{\prime} \rightarrow Y$, $\left.\alpha\right|_{Y^{\prime}}: Y^{\prime} \rightarrow X$ are étale. We also require that $\left.\Phi \circ \alpha\right|_{Y^{\prime}}=\left.\jmath\right|_{Y^{\prime}}: Y^{\prime} \rightarrow Y$.

(ii) Write $N_{U V}$ for the normal bundle of $\Phi(U)$ in $V$, regarded as an algebraic vector bundle on $U$ in the exact sequence of vector bundles on $U$ :

$$
0 \longrightarrow T U \stackrel{\mathrm{d} \Phi}{\longrightarrow} \Phi^{*}(T U) \stackrel{\Pi_{U V}}{\longrightarrow} N_{U V} \longrightarrow 0
$$

Then there exists a unique $q_{U V} \in H^{0}\left(\left.S^{2} N_{U V}^{*}\right|_{X}\right)$ which is a nondegenerate quadratic form on $\left.N_{U V}\right|_{X}$, such that whenever $U^{\prime}, V^{\prime}, \iota, \jmath, \Phi^{\prime}, \beta, n, X^{\prime}$ are as in (i), writing $\left\langle\mathrm{d} z_{1}, \ldots, \mathrm{d} z_{n}\right\rangle_{U^{\prime}}$ for the trivial vector bundle on $U^{\prime}$ with basis $\mathrm{d} z_{1}, \ldots, \mathrm{d} z_{n}$, there is a natural isomorphism $\hat{\beta}:\left\langle\mathrm{d} z_{1}, \ldots, \mathrm{d} z_{n}\right\rangle_{U^{\prime}} \rightarrow \iota^{*}\left(N_{U V}^{*}\right)$ making the following diagram commute:

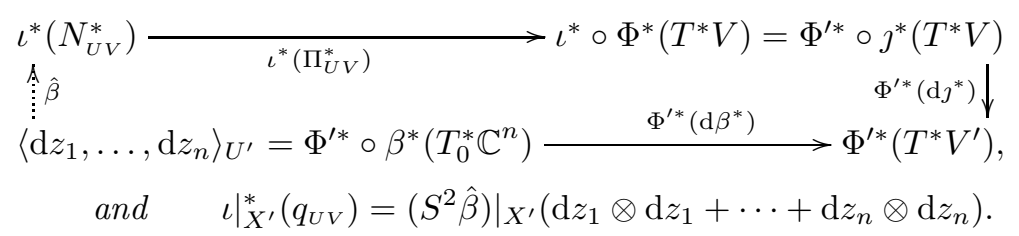


(iii) Now suppose $W$ is another smooth $\mathbb{C}$-scheme, $h: W \rightarrow \mathbb{C}$ is regular, $Z=\operatorname{Crit}(h)$ as a $\mathbb{C}$-subscheme of $W$, and $\Psi: V \hookrightarrow W$ is a closed embedding of $\mathbb{C}$-schemes with $g=h \circ \Psi: V \rightarrow \mathbb{C}$ and $\left.\Psi\right|_{Y}: Y \rightarrow Z$ an isomorphism. Define $N_{V W}, q_{V W}$ and $N_{U W}, q_{U W}$ using $\Psi: V \hookrightarrow W$ and $\Psi \circ \Phi: U \hookrightarrow W$ as in (ii) above. Then there are unique morphisms $\gamma_{U V W}, \delta_{U V W}$ which make the following diagram of vector bundles on $U$ commute, with straight lines exact:

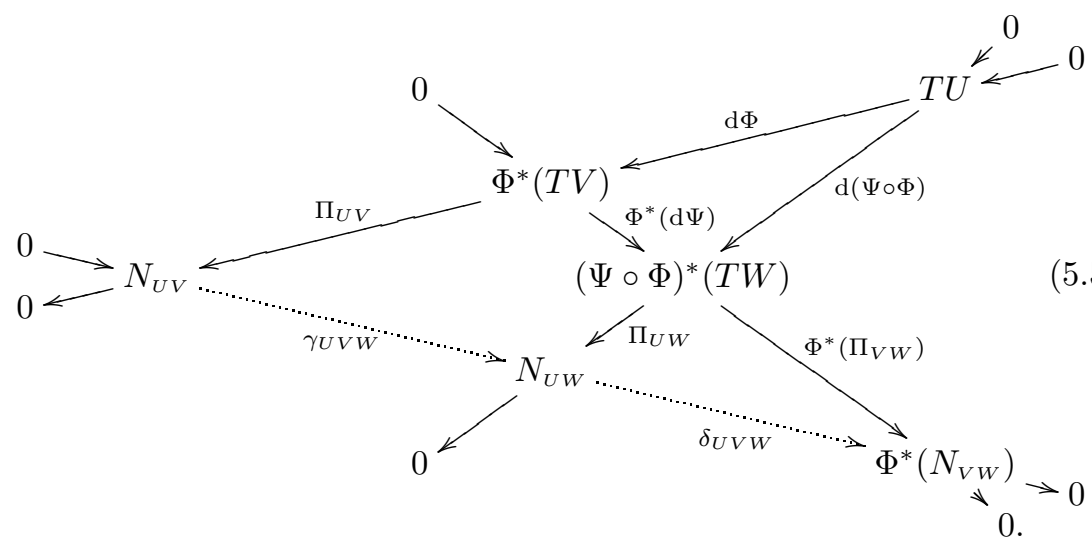

Restricting to $X$ gives an exact sequence of vector bundles:

$$
\left.\left.\left.0 \rightarrow N_{U V}\right|_{X} \stackrel{\left.\gamma_{U V W}\right|_{X}}{\longrightarrow} N_{U W}\right|_{X} \stackrel{\left.\delta_{U V W}\right|_{X}}{\longrightarrow} \Phi\right|_{X} ^{*}\left(N_{V W}\right) \rightarrow 0 .
$$

Then there is a natural isomorphism of vector bundles on $X$

$$
\left.\left.\left.N_{U W}\right|_{X} \cong N_{U V}\right|_{X} \oplus \Phi\right|_{X} ^{*}\left(N_{V W}\right),
$$

compatible with the exact sequence (5.6), which identifies

$$
\begin{aligned}
q_{U W} & \left.\cong q_{U V} \oplus \Phi\right|_{X} ^{*}\left(q_{V W}\right) \oplus 0 \quad \text { under the splitting } \\
\left.S^{2} N_{U W}\right|_{X} ^{*} & \left.\left.\cong S^{2} N_{U V}\right|_{X} ^{*} \oplus \Phi\right|_{X} ^{*}\left(\left.S^{2} N_{V W}^{*}\right|_{Y}\right) \oplus\left(\left.\left.N_{U V}^{*}\right|_{X} \otimes \Phi\right|_{X} ^{*}\left(N_{V W}^{*}\right)\right) .
\end{aligned}
$$

(iv) Analogues of (i)-(iii) hold for complex analytic spaces, replacing the smooth $\mathbb{C}$-schemes $U, V, W$ by complex manifolds, the regular functions $f, g, h$ by holomorphic functions, the $\mathbb{C}$-schemes $X, Y, Z$ by complex analytic spaces, the étale open sets $\iota: U^{\prime} \rightarrow U, \jmath: V^{\prime} \rightarrow V$ by complex analytic open sets $U^{\prime} \subseteq U$, $V^{\prime} \subseteq V$, and with $\alpha \times \beta: V^{\prime} \rightarrow U \times \mathbb{C}^{n}$ a biholomorphism with a complex analytic open neighbourhood of $(x, 0)$ in $U \times \mathbb{C}^{n}$.

Following [23, Def.s $2.26 \& 2.34]$, we define:

Definition 5.2. Let $U, V$ be smooth $\mathbb{C}$-schemes, $f: U \rightarrow \mathbb{C}, g: V \rightarrow \mathbb{C}$ be regular, and $X=\operatorname{Crit}(f), Y=\operatorname{Crit}(g)$ as $\mathbb{C}$-subschemes of $U, V$. Suppose $\Phi: U \hookrightarrow V$ is a closed embedding of $\mathbb{C}$-schemes with $f=g \circ \Phi: U \rightarrow \mathbb{C}$ and $\left.\Phi\right|_{X}: X \rightarrow Y$ an isomorphism. Then Theorem [5.1(ii) defines the normal 
bundle $N_{U V}$ of $U$ in $V$, a vector bundle on $U$ of $\operatorname{rank} n=\operatorname{dim} V-\operatorname{dim} U$, and a nondegenerate quadratic form $q_{U V} \in H^{0}\left(\left.S^{2} N_{U V}^{*}\right|_{X}\right)$. Taking top exterior powers in the dual of (5.2) gives an isomorphism of line bundles on $U$

$$
\rho_{U V}: K_{U} \otimes \Lambda^{n} N_{U V}^{*} \stackrel{\cong}{\longrightarrow} \Phi^{*}\left(K_{V}\right),
$$

where $K_{U}, K_{V}$ are the canonical bundles of $U, V$.

Write $X^{\text {red }}$ for the reduced $\mathbb{C}$-subscheme of $X$. As $q_{U V}$ is a nondegenerate quadratic form on $\left.N_{V W}\right|_{X}$, its determinant $\operatorname{det}\left(q_{V W}\right)$ is a nonzero section of $\left.\left(\Lambda^{n} N_{V W}^{*}\right)\right|_{X} ^{\otimes^{2}}$. Define an isomorphism of line bundles on $X^{\text {red: }}$

$$
J_{\Phi}=\rho_{U V}^{\otimes^{2}} \circ\left(\left.\operatorname{id}_{\left.K_{U}^{2}\right|_{X^{\text {red }}}} \otimes \operatorname{det}\left(q_{U V}\right)\right|_{X^{\text {red }}}\right):\left.\left.K_{U}^{\otimes^{2}}\right|_{X^{\text {red }}} \stackrel{\cong}{\longrightarrow} \Phi\right|_{X^{\text {red }}} ^{*}\left(K_{V}^{\otimes^{2}}\right) .
$$

Since principal $\mathbb{Z} / 2 \mathbb{Z}$-bundles $\pi: P \rightarrow X$ in the sense of Definition 2.9 are an (étale or complex analytic) topological notion, and $X^{\text {red }}$ and $X$ have the same topological space (even in the étale or complex analytic topology), principal $\mathbb{Z} / 2 \mathbb{Z}$-bundles on $X^{\text {red }}$ and on $X$ are equivalent. Define $\pi_{\Phi}: P_{\Phi} \rightarrow X$ to be the principal $\mathbb{Z} / 2 \mathbb{Z}$-bundle which parametrizes square roots of $J_{\Phi}$ on $X^{\text {red }}$. That is, (étale or complex analytic) local sections $s_{\alpha}: X \rightarrow P_{\Phi}$ of $P_{\Phi}$ correspond to local isomorphisms $\alpha:\left.\left.K_{U}\right|_{X^{\text {red }}} \rightarrow \Phi\right|_{X \text { red }} ^{*}\left(K_{V}\right)$ on $X^{\text {red }}$ with $\alpha \otimes \alpha=J_{\Phi}$.

Now suppose $W$ is another smooth $\mathbb{C}$-scheme, $h: W \rightarrow \mathbb{C}$ is regular, $Z=$ $\operatorname{Crit}(h)$ as a $\mathbb{C}$-subscheme of $W$, and $\Psi: V \hookrightarrow W$ is a closed embedding of $\mathbb{C}$-schemes with $g=h \circ \Psi: V \rightarrow \mathbb{C}$ and $\left.\Psi\right|_{Y}: Y \rightarrow Z$ an isomorphism. Then Theorem 5.1(iii) applies, and from (5.7)-(5.8) we can deduce that

$$
\begin{aligned}
& J_{\Psi \circ \Phi}=\left.\Phi\right|_{X^{\text {red }}} ^{*}\left(J_{\Psi}\right) \circ J_{\Phi}:\left.\left.K_{U}^{\otimes^{2}}\right|_{X^{\text {red }}} \stackrel{\cong}{\longrightarrow}(\Psi \circ \Phi)\right|_{X^{\text {red }}} ^{*}\left(K_{W}^{\otimes^{2}}\right) \\
&=\left.\Phi\right|_{X^{\text {red }}} ^{*}\left[\left.\Psi\right|_{Y^{\text {red }}} ^{*}\left(K_{W}^{\otimes^{2}}\right)\right] .
\end{aligned}
$$

For the principal $\mathbb{Z} / 2 \mathbb{Z}$-bundles $\pi_{\Phi}: P_{\Phi} \rightarrow X, \pi_{\Psi}: P_{\Psi} \rightarrow Y, \pi_{\Psi \circ \Phi}: P_{\Psi \circ \Phi} \rightarrow X$, equation (5.10) implies that there is a canonical isomorphism

$$
\Xi_{\Psi, \Phi}:\left.P_{\Psi \circ \Phi} \stackrel{\cong}{\longrightarrow} \Phi\right|_{X} ^{*}\left(P_{\Psi}\right) \otimes_{\mathbb{Z} / 2 \mathbb{Z}} P_{\Phi}
$$

It is also easy to see that these $\Xi_{\Psi, \Phi}$ have an associativity property under triple compositions, that is, given another smooth $\mathbb{C}$-scheme $T$, regular $e: T \rightarrow \mathbb{C}$ with $Q:=\operatorname{Crit}(e)$, and $\Upsilon: T \hookrightarrow U$ a closed embedding with $e=f \circ \Upsilon: T \rightarrow \mathbb{C}$ and $\left.\Upsilon\right|_{Q}: Q \rightarrow X$ an isomorphism, then

$$
\begin{array}{r}
\left(\operatorname{id}_{\left.(\Phi \circ \Upsilon)\right|_{Q} ^{*}\left(P_{\Psi}\right)} \otimes \Xi_{\Phi, \Upsilon}\right) \circ \Xi_{\Psi, \Phi \circ \Upsilon}=\left(\left.\Upsilon\right|_{Q} ^{*}\left(\Xi_{\Psi, \Phi}\right) \otimes \operatorname{id}_{P_{\Upsilon}}\right) \circ \Xi_{\Psi \circ \Phi, \Upsilon}: \\
\left.\left.P_{\Psi \circ \Phi \circ \Upsilon} \longrightarrow(\Phi \circ \Upsilon)\right|_{Q} ^{*}\left(P_{\Psi}\right) \otimes_{\mathbb{Z} / 2 \mathbb{Z}} \Upsilon\right|_{Q} ^{*}\left(P_{\Phi}\right) \otimes_{\mathbb{Z} / 2 \mathbb{Z}} P_{\Upsilon} .
\end{array}
$$

Analogues of all the above also work for complex manifolds and complex analytic spaces, as in Theorem 5.1(v).

The reason for restricting to $X^{\text {red }}$ above is the following [23, Prop. 2.27], whose proof uses the fact that $X^{\text {red }}$ is reduced in an essential way. 
Lemma 5.3. In Definition 5.2, the isomorphism $J_{\Phi}$ in (5.9) and the principal $\mathbb{Z} / 2 \mathbb{Z}$-bundle $\pi_{\Phi}: P_{\Phi} \rightarrow X$ depend only on $U, V, X, Y, f, g$ and $\left.\Phi\right|_{X}: X \rightarrow Y$. That is, they do not depend on $\Phi: U \rightarrow V$ apart from $\left.\Phi\right|_{X}: X \rightarrow Y$.

Using the notation of Definition 5.2, we can state our main result:

Theorem 5.4. (a) Let $U, V$ be smooth $\mathbb{C}$-schemes, $f: U \rightarrow \mathbb{C}, g: V \rightarrow \mathbb{C}$ be regular, and $X=\operatorname{Crit}(f), Y=\operatorname{Crit}(g)$ as $\mathbb{C}$-subschemes of $U, V$. Let $\Phi: U \hookrightarrow$ $V$ be a closed embedding of $\mathbb{C}$-schemes with $f=g \circ \Phi: U \rightarrow \mathbb{C}$, and suppose $\left.\Phi\right|_{X}: X \rightarrow V \supseteq Y$ is an isomorphism $\left.\Phi\right|_{X}: X \rightarrow Y$. Then there is a natural isomorphism of perverse sheaves on $X$ :

$$
\Theta_{\Phi}:\left.\mathcal{P} \mathcal{V}_{U, f}^{\bullet} \longrightarrow \Phi\right|_{X} ^{*}\left(\mathcal{P} \mathcal{V}_{V, g}^{\bullet}\right) \otimes_{\mathbb{Z} / 2 \mathbb{Z}} P_{\Phi}
$$

where $\mathcal{P} \mathcal{V}_{U, f}^{\bullet}, \mathcal{P} \mathcal{V}_{V, q}^{\bullet}$ are the perverse sheaves of vanishing cycles from $₫ 2.4$, and $P_{\Phi}$ the principal $\mathbb{Z} / 2 \mathbb{Z}$-bundle from Definition 5.2 and if $\mathcal{Q}^{\bullet}$ is a perverse sheaf on $X$ then $\mathcal{Q}^{\bullet} \otimes_{\mathbb{Z} / 2 \mathbb{Z}} P_{\Phi}$ is as in Definition 2.9. Also the following diagrams commute, where $\sigma_{U, f}, \sigma_{V, g}, \tau_{U, f}, \tau_{V, g}$ are as in (2.6)-(2.7):

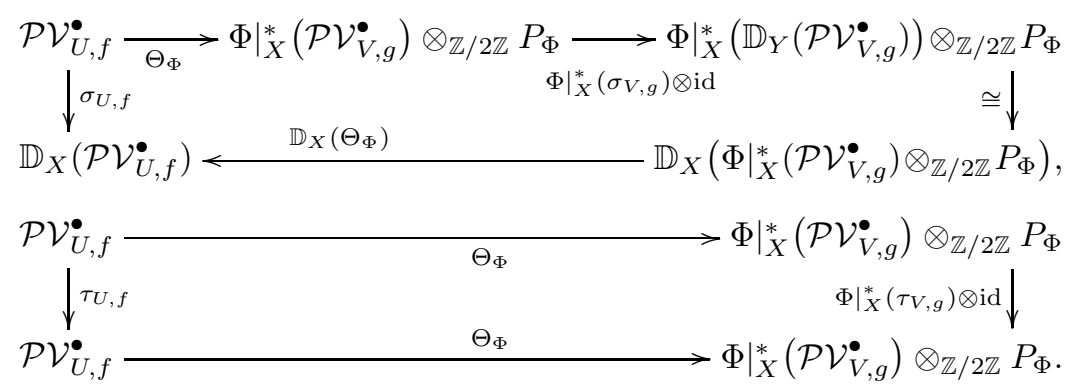

If $U=V, f=g, \Phi=\mathrm{id}_{U}$ then $\pi_{\Phi}: P_{\Phi} \rightarrow X$ is trivial, and $\Theta_{\Phi}$ corresponds to $\operatorname{id}_{\mathcal{P} V_{U, f}^{\bullet}}$ under the natural isomorphism $\operatorname{id}_{X}^{*}\left(\mathcal{P} \mathcal{V}_{U, f}^{\bullet}\right) \otimes_{\mathbb{Z} / 2 \mathbb{Z}} P_{\Phi} \cong \mathcal{P} \mathcal{V}_{U, f}^{\bullet}$.

(b) The isomorphism $\Theta_{\Phi}$ in (5.13) depends only on $U, V, X, Y, f, g$ and $\left.\Phi\right|_{X}$ : $X \rightarrow Y$. That is, if $\tilde{\Phi}: U \rightarrow V$ is an alternative choice for $\Phi$ with $\left.\Phi\right|_{X}=\left.\tilde{\Phi}\right|_{X}$ : $X \rightarrow Y$, then $\Theta_{\Phi}=\Theta_{\tilde{\Phi}}$, noting that $P_{\Phi}=P_{\tilde{\Phi}}$ by Lemma [5.3,

(c) Now suppose $W$ is another smooth $\mathbb{C}$-scheme, $h: W \rightarrow \mathbb{C}$ is regular, $Z=$ $\operatorname{Crit}(h)$, and $\Psi: V \hookrightarrow W$ is a closed embedding with $g=h \circ \Psi: V \rightarrow \mathbb{C}$ and $\left.\Psi\right|_{Y}: Y \rightarrow Z$ an isomorphism. Then Definition 5.2 defines principal $\mathbb{Z} / 2 \mathbb{Z}$ bundles $\pi_{\Phi}: P_{\Phi} \rightarrow X, \pi_{\Psi}: P_{\Psi} \rightarrow Y, \pi_{\Psi \circ \Phi}: P_{\Psi \circ \Phi} \rightarrow X$ and an isomorphism $\Xi_{\Psi, \Phi}$ in (5.11), and part (a) defines isomorphisms of perverse sheaves $\Theta_{\Phi}, \Theta_{\Psi \circ \Phi}$ on $X$ and $\Theta_{\Psi}$ on $Y$. Then the following commutes in $\operatorname{Perv}(X)$ :

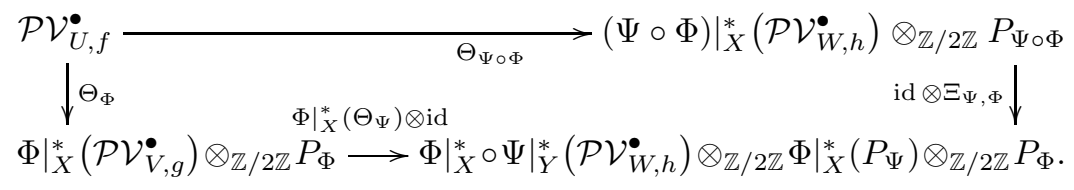

(d) The analogues of (a)-(c) also hold for $\mathscr{D}$-modules on $\mathbb{C}$-schemes, for perverse sheaves and $\mathscr{D}$-modules on complex analytic spaces, and for mixed Hodge modules on $\mathbb{C}$-schemes and complex analytic spaces, as in $\$ 2.6-\$ 2.10$, 
Example 5.5. Let $U=\mathbb{C} \backslash\{0\}$ and $V=(\mathbb{C} \backslash\{0\}) \times \mathbb{C}$ as smooth $\mathbb{C}$-schemes, define regular $f: U \rightarrow \mathbb{C}$ and $g: V \rightarrow \mathbb{C}$ by $f(x)=0$ and $g(x, y)=x^{k} y^{2}$ for fixed $k \in \mathbb{Z}$, and define $\Phi: U \rightarrow V$ by $\Phi: x \mapsto(x, 0)$, so that $f=g \circ \Phi: U \rightarrow \mathbb{C}$. Then $X:=\operatorname{Crit}(f)=U$, and $Y:=\operatorname{Crit}(g)=\left\{(x, y) \in V: k x^{k-1} y^{2}=2 x^{k} y=\right.$ $0\}=\{(x, y) \in V: y=0\}$, as $x \neq 0$. Thus $\left.\Phi\right|_{X}: X \rightarrow Y$ is an isomorphism.

In Theorem 5.1(ii), $N_{U V}^{*}$ is the trivial line bundle on $U$ with basis $\mathrm{d} y$, and $q_{U V}=x^{k} \mathrm{~d} y \otimes \mathrm{d} y$. In Definition [5.2, $\left.K_{U}\right|_{X}$ and $\left.\Phi\right|_{X} ^{*}\left(K_{V}\right)$ are the trivial line bundles on $X=X^{\text {red }}=U$ with bases $\mathrm{d} x$ and $\mathrm{d} x \wedge \mathrm{d} y$, and $J_{\Phi}$ in (5.9) maps

$$
J_{\Phi}: \mathrm{d} x \otimes \mathrm{d} x \longmapsto x^{k}(\mathrm{~d} x \wedge \mathrm{d} y) \otimes(\mathrm{d} x \wedge \mathrm{d} y) .
$$

The principal $\mathbb{Z} / 2 \mathbb{Z}$-bundle $\pi_{\Phi}: P_{\Phi} \rightarrow X$ in Definition 5.2 parametrizes $\alpha$ : $\left.\left.K_{U}\right|_{X} \rightarrow \Phi\right|_{X} ^{*}\left(K_{V}\right)$ with $\alpha \otimes \alpha=J_{\Phi}$. Writing $\alpha: \mathrm{d} x \mapsto p \mathrm{~d} x \wedge \mathrm{d} y$ for $p$ a local function on $X=\mathbb{C} \backslash\{0\}, \alpha \otimes \alpha=J_{\Phi}$ reduces to $p^{2}=x^{k}$. Thus, $P_{\Phi}$ parametrizes (étale local) square roots $p$ of $x^{k}: \mathbb{C} \backslash\{0\} \rightarrow \mathbb{C} \backslash\{0\}$.

If $k$ is even then $x^{k}$ has a global square root $p=x^{k / 2}$, so the principal $\mathbb{Z} / 2 \mathbb{Z}$ bundle $P_{\Phi}$ has a global section, and is trivial. If $k$ is odd then $x^{k}$ has no global square root on $X=\mathbb{C} \backslash\{0\}$, so $P_{\Phi}$ has no global section, and is nontrivial.

Thus, Theorem 5.4 implies that if $k$ is even then $\mathcal{P} \mathcal{V}_{V, g}^{\bullet} \cong A_{Y}[1]$ is the constant perverse sheaf on $Y$, but if $k$ is odd then $\mathcal{P} \mathcal{V}_{V, g}^{\bullet}$ is the twist of $A_{Y}$ [1] by the unique nontrivial principal $\mathbb{Z} / 2 \mathbb{Z}$-bundle on $Y \cong \mathbb{C} \backslash\{0\}$.

\subsection{Theorem 5.4(a): the isomorphism $\Theta_{\Phi}$}

Let $U, V, f, g, X, Y, \Phi$ be as in Theorem 5.4 (a), and use the notation $N_{U V}, q_{U V}$ from Theorem [5.1(ii) and $J_{\Phi}, P_{\Phi}$ from Definition 5.2. We will show that there exists a unique perverse sheaf morphism $\Theta_{\Phi}$ in (5.13) which is characterized by the property that whenever $U^{\prime}, V^{\prime}, \iota, \jmath, \Phi^{\prime}, \alpha, \beta, X^{\prime}, Y^{\prime}, f^{\prime}, g^{\prime}$ are as in Theorem 5.1(i) then the following diagram of isomorphisms in $\operatorname{Perv}\left(X^{\prime}\right)$ commutes:

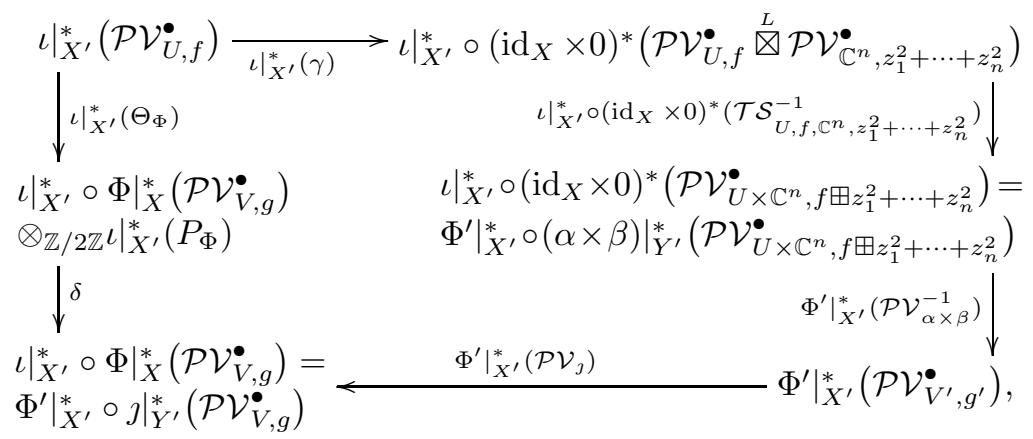

where $\mathcal{T} \mathcal{S}_{U, f, \mathbb{C}^{n}, z_{1}^{2}+\cdots+z_{n}^{2}}$ is as in (2.8), and $\gamma, \delta$ are defined as follows:

(A) $\gamma: \mathcal{P} \mathcal{V}_{U, f}^{\bullet} \rightarrow\left(\operatorname{id}_{X} \times 0\right)^{*}\left(\mathcal{P} \mathcal{V}_{U, f}^{\bullet} \stackrel{L}{\otimes} \mathcal{P} \mathcal{V}_{\mathbb{C}^{n}, z_{1}^{2}+\cdots+z_{n}^{2}}^{\bullet}\right.$ in $\operatorname{Perv}(X)$ comes from the isomorphism $\mathcal{P} \mathcal{V}_{\mathbb{C}^{n}, z_{1}^{2}+\cdots+z_{n}^{2}}^{\bullet} A_{\{0\}}$ in (2.12). 
(B) The principal $\mathbb{Z} / 2 \mathbb{Z}$-bundle $P_{\Phi} \rightarrow X$ comes from $\left(\left.N_{U V}\right|_{X^{\text {red }}},\left.q_{U V}\right|_{X^{\text {red }}}\right)$, as the bundle of square roots of $\operatorname{det}\left(\left.q_{U V}\right|_{X^{\text {red }}}\right)$. Thus, the pullback $\left.\iota\right|_{X^{\prime}} ^{*}\left(P_{\Phi}\right) \rightarrow$ $X^{\prime}$ comes from $\left(\left.\iota\right|_{X^{\prime \text { red }}} ^{*}\left(N_{U V}\right),\left.\iota\right|_{X^{\prime \text { red }}} ^{*}\left(q_{U V}\right)\right)$. Now Theorem [5.1(ii) defines $\left.\hat{\beta}\right|_{X^{\prime \text { red }}}:\left\langle\mathrm{d} z_{1}, \ldots, \mathrm{d} z_{n}\right\rangle_{X^{\prime \text { red }}} \stackrel{\cong}{\longrightarrow} \iota_{X^{\prime \text { red }}}^{*}\left(N_{U V}^{*}\right)$ identifying $\sum_{j=1}^{n} \mathrm{~d} z_{j}^{2}$ with $\iota_{X^{\text {red }}}^{*}\left(q_{U V}\right)$. Thus, $\left.\hat{\beta}\right|_{X^{\prime \text { red }}}$ induces a trivialization of $\iota_{X^{\prime}}^{*}\left(P_{\Phi}\right) \rightarrow X^{\prime}$.

Then $\delta:\left.\left.\left.\left.\iota\right|_{X^{\prime}} ^{*} \circ \Phi\right|_{X} ^{*}\left(\mathcal{P} \mathcal{V}_{V, g}^{\bullet}\right) \otimes_{\mathbb{Z} / 2 \mathbb{Z}} \iota_{X^{\prime}}^{*}\left(P_{\Phi}\right) \rightarrow \iota\right|_{X^{\prime}} ^{*} \circ \Phi\right|_{X} ^{*}\left(\mathcal{P} \mathcal{V}_{V, g}^{\bullet}\right)$ in $\operatorname{Perv}\left(X^{\prime}\right)$ comes from this trivialization of the principal $\mathbb{Z} / 2 \mathbb{Z}$-bundle $\left.\iota\right|_{X^{\prime}} ^{*}\left(P_{\Phi}\right) \rightarrow X^{\prime}$.

Since Theorem 5.1(i) holds for each $x \in X$, we may choose a family $\left\{\left(U_{a}^{\prime}\right.\right.$, $\left.\left.V_{a}^{\prime}, \iota_{a}, \jmath_{a}, \Phi_{a}^{\prime}, \alpha_{a}, \beta_{a}, f_{a}^{\prime}, g_{a}^{\prime}, X_{a}^{\prime}, Y_{a}^{\prime}\right): a \in A\right\}$, such that $U_{a}^{\prime}, V_{a}^{\prime}, \ldots, Y_{a}^{\prime}$ satisfy Theorem 5.1(i) for each $a \in A$, and $\left\{\left.\iota_{a}^{\prime}\right|_{X_{a}^{\prime}}: X_{a}^{\prime} \rightarrow X\right\}_{a \in A}$ is an étale open cover of $X$. For each $a \in A$, define an isomorphism

$$
\Theta_{a}:\left.\left.\left.\iota_{a}\right|_{X_{a}^{\prime}} ^{*}\left(\mathcal{P} \mathcal{V}_{U, f}^{\bullet}\right) \longrightarrow \iota_{a}\right|_{X_{a}^{\prime}} ^{*} \circ \Phi\right|_{X} ^{*}\left(\mathcal{P} \mathcal{V}_{V, g}^{\bullet}\right)
$$

to make the following diagram of isomorphisms commute:

$$
\begin{aligned}
& \left.\left.\iota_{a}\right|_{X_{a}^{\prime}} ^{*}\left(\mathcal{P} \mathcal{V}_{U, f}^{\bullet}\right) \underset{\left.\iota_{a}\right|_{X_{a}^{\prime}} ^{*}(\gamma)}{\longrightarrow} \iota_{a}\right|_{X_{a}^{\prime}} ^{*} \circ\left(\operatorname{id}_{X} \times 0\right)^{*}\left(\mathcal{P} \mathcal{V}_{U, f}^{\bullet} \stackrel{L}{\otimes} \mathcal{P} \mathcal{V}_{\mathbb{C}^{n}, z_{1}^{2}+\cdots+z_{n}^{2}}^{\bullet}\right) \\
& \left.\downarrow \Theta_{a} \quad \iota_{a}\right|_{X_{a}^{\prime}} ^{*}{ }^{\circ}(\mathrm{id} X \times 0)^{*}\left(\mathcal{T S}_{U, f, \mathrm{C}^{n}, z_{1}^{2}+\cdots+z_{n}^{2}}^{-1} \downarrow\right. \\
& \left.\left.\left.\iota_{a}\right|_{X_{a}^{\prime}} ^{*} \circ \Phi\right|_{X} ^{*}\left(\mathcal{P} \mathcal{V}_{V, g}^{\bullet}\right) \quad \iota_{a}\right|_{X_{a}^{\prime}} ^{*} \circ\left(\operatorname{id}_{X} \times 0\right)^{*}\left(\mathcal{P} \mathcal{V}_{U \times \mathbb{C}^{n}, f \boxplus z_{1}^{2}+\cdots+z_{n}^{2}}^{\bullet}\right)= \\
& \left.\left.\left.\otimes_{\mathbb{Z} / 2 \mathbb{Z}} \iota_{a}\right|_{X_{a}^{\prime}} ^{*}\left(P_{\Phi}\right) \quad \Phi_{a}^{\prime}\right|_{X_{a}^{\prime}} ^{*} \circ\left(\alpha_{a} \times \beta_{a}\right)\right|_{Y_{a}^{\prime}} ^{*}\left(\mathcal{P} \mathcal{V}_{U \times \mathbb{C}^{n}, f \boxplus z_{1}^{2}+\cdots+z_{n}^{2}}^{\bullet}\right) \\
& \left.\left.\downarrow^{\delta_{a}} \quad \Phi^{\prime}\right|_{X_{a}^{\prime}} ^{*}\left(\mathcal{P} \mathcal{V}_{j a}\right) \quad \Phi_{a}^{\prime}\right|_{X_{a}^{\prime}} ^{*}\left(\mathcal{P} \mathcal{V}_{\alpha_{a} \times \beta_{a}}^{-1}\right) \mid \\
& \left.\begin{array}{l}
\left.\left.\iota_{a}\right|_{X_{a}^{\prime}} ^{*} \circ \Phi\right|_{X} ^{*}\left(\mathcal{P} \mathcal{V}_{V, g}^{\bullet}\right)=\left.\Phi_{a}^{\prime}\right|_{X_{a}^{\prime}} ^{*}\left(\mathcal{P} \mathcal{V}_{\jmath_{a}}\right) \\
\left.\left.\Phi_{a}^{\prime}\right|_{X_{a}^{\prime}} ^{*} \circ \jmath_{a}\right|_{Y_{a}^{\prime}} ^{*}\left(\mathcal{P} \mathcal{V}_{V, g}^{\bullet}\right)
\end{array} \Phi_{a}^{\prime}\right|_{X_{a}^{\prime}} ^{*}\left(\mathcal{P} \mathcal{V}_{V_{a}^{\prime}, g_{a}^{\prime}}^{\bullet}\right),
\end{aligned}
$$

where $\gamma$ is as in (A), and $\delta_{a}$ defined as in (B) above.

For $a, b \in A$, define $U_{a b}^{\prime}=U_{a}^{\prime} \times_{\iota_{a}, U, \iota_{b}} U_{b}^{\prime}$ and $V_{a b}^{\prime}=V_{a}^{\prime} \times_{\jmath_{a}, V, \jmath_{b}} V_{b}^{\prime}$, with projections $\Pi_{U_{a}^{\prime}}: U_{a b}^{\prime} \rightarrow U_{a}^{\prime}, \Pi_{U_{b}^{\prime}}: U_{a b}^{\prime} \rightarrow U_{b}^{\prime}, \Pi_{V_{a}^{\prime}}: V_{a b}^{\prime} \rightarrow V_{a}^{\prime}, \Pi_{V_{b}^{\prime}}: V_{a b}^{\prime} \rightarrow V_{b}^{\prime}$. Then $U_{a b}^{\prime}, V_{a b}^{\prime}$ are smooth and $\Pi_{U_{a}^{\prime}}, \Pi_{U_{b}^{\prime}}, \Pi_{V_{a}^{\prime}}, \Pi_{V_{b}^{\prime}}$ étale. The universal property of $V_{a}^{\prime} \times_{\jmath_{a}, V, \jmath_{b}} V_{b}^{\prime}$ gives a unique morphism $\Phi_{a b}^{\prime}: U_{a b}^{\prime} \rightarrow V_{a b}^{\prime}$ with

$$
\Pi_{V_{a}^{\prime}} \circ \Phi_{a b}^{\prime}=\Phi_{a}^{\prime} \circ \Pi_{U_{a}^{\prime}} \text { and } \Pi_{V_{b}^{\prime}} \circ \Phi_{a b}^{\prime}=\Phi_{b}^{\prime} \circ \Pi_{U_{b}^{\prime}} .
$$

Set $f_{a b}^{\prime}=f_{a}^{\prime} \circ \Pi_{U_{a}^{\prime}}: U_{a b}^{\prime} \rightarrow \mathbb{C}, g_{a b}^{\prime}=g_{a}^{\prime} \circ \Pi_{V_{a}^{\prime}}: V_{a b}^{\prime} \rightarrow \mathbb{C}$ and $X_{a b}^{\prime}=$ $\operatorname{Crit}\left(f_{a b}^{\prime}\right) \subseteq U_{a b}^{\prime}, Y_{a b}^{\prime}=\operatorname{Crit}\left(g_{a b}^{\prime}\right) \subseteq V_{a b}^{\prime}$. As for (4.17) we have

$$
\begin{aligned}
f_{a b}^{\prime} & =f_{a}^{\prime} \circ \Pi_{U_{a}^{\prime}}=f \circ \iota_{a} \circ \Pi_{U_{a}^{\prime}}=f \circ \iota_{b} \circ \Pi_{U_{b}^{\prime}}=f_{b}^{\prime} \circ \Pi_{U_{b}^{\prime}}, \\
g_{a b}^{\prime} & =g_{a}^{\prime} \circ \Pi_{V_{a}^{\prime}}=g \circ \jmath_{a} \circ \Pi_{V_{a}^{\prime}}=g \circ \jmath_{b} \circ \Pi_{V_{b}^{\prime}}=g_{b}^{\prime} \circ \Pi_{V_{b}^{\prime}} \\
& =\left(f \boxplus z_{1}^{2}+\cdots+z_{n}^{2}\right) \circ\left(\alpha_{a} \times \beta_{a}\right) \circ \Pi_{V_{a}^{\prime}} \\
& =\left(f \boxplus z_{1}^{2}+\cdots+z_{n}^{2}\right) \circ\left(\alpha_{b} \times \beta_{b}\right) \circ \Pi_{V_{b}^{\prime}} .
\end{aligned}
$$


Apply Theorem 3.1] with $V_{a b}^{\prime}, U \times \mathbb{C}^{n},\left(\alpha_{a} \times \beta_{a}\right) \circ \Pi_{V_{a}^{\prime}},\left(\alpha_{b} \times \beta_{b}\right) \circ \Pi_{V_{b}^{\prime}}, g_{a b}^{\prime}$, and $f \boxplus z_{1}^{2}+\cdots+z_{n}^{2}$ in place of $V, W, \Phi, \Psi, f, g$. The analogue of $\left.\Phi\right|_{X}=\left.\Psi\right|_{X}$ is

$$
\begin{aligned}
\left(\alpha_{a}\right. & \left.\times \beta_{a}\right)\left.\circ \Pi_{V_{a}^{\prime}}\right|_{Y_{a b}^{\prime}}=\left.\left(\left.\left(\left.\left.\Phi\right|_{X} ^{-1} \circ \Phi\right|_{X} \circ \alpha_{a}\right)\right|_{Y_{a}^{\prime}} \times 0\right) \circ \Pi_{V_{a}^{\prime}}\right|_{Y_{a b}^{\prime}} \\
& =\left(\left.\left.\Phi\right|_{X} ^{-1} \circ \jmath_{a} \circ \Pi_{V_{a}^{\prime}}\right|_{Y_{a b}^{\prime}}\right) \times 0=\left(\left.\left.\Phi\right|_{X} ^{-1} \circ \jmath_{b} \circ \Pi_{V_{b}^{\prime}}\right|_{Y_{a b}^{\prime}}\right) \times 0 \\
& =\left.\left(\left.\left(\left.\left.\Phi\right|_{X} ^{-1} \circ \Phi\right|_{X} \circ \alpha_{b}\right)\right|_{Y_{b}^{\prime}} \times 0\right) \circ \Pi_{V_{b}^{\prime}}\right|_{Y_{a b}^{\prime}}=\left.\left(\alpha_{b} \times \beta_{b}\right) \circ \Pi_{V_{b}^{\prime}}\right|_{Y_{a b}^{\prime}},
\end{aligned}
$$

using $\left.\Phi\right|_{X}: X \rightarrow Y$ an isomorphism and $\left.\beta_{a}\right|_{Y_{a}^{\prime}}=0$ in the first step, $\left.\jmath_{a}\right|_{Y_{a}^{\prime}}=$ $\left.\left.\Phi\right|_{X} \circ \alpha_{a}\right|_{Y_{a}^{\prime}}$ in the second, $\jmath_{a} \circ \Pi_{V_{a}^{\prime}}=\jmath_{b} \circ \Pi_{V_{b}^{\prime}}$ in the third, $\left.\jmath_{b}\right|_{Y_{b}^{\prime}}=\left.\left.\Phi\right|_{X} \circ \alpha_{b}\right|_{Y_{b}^{\prime}}$ in the fourth, and $\left.\beta_{b}\right|_{Y_{b}^{\prime}}=0$ in the fifth. Thus Theorem 3.1 gives

$$
\begin{gathered}
\mathcal{P} \mathcal{V}_{\left(\alpha_{a} \times \beta_{a}\right) \circ \Pi_{V_{a}^{\prime}}}=\operatorname{det}\left[\left.\left.\mathrm{d}\left(\left(\alpha_{b} \times \beta_{b}\right) \circ \Pi_{V_{b}^{\prime}}\right)\right|_{Y_{a b}^{\text {red }}} ^{-1} \circ \mathrm{d}\left(\left(\alpha_{a} \times \beta_{a}\right) \circ \Pi_{V_{a}^{\prime}}\right)\right|_{Y_{a b}^{\prime r e d}}\right] \\
\mathcal{P} \mathcal{V}_{\left(\alpha_{b} \times \beta_{b}\right) \circ \Pi_{V_{b}^{\prime}}}:\left.\mathcal{P} \mathcal{V}_{V_{a b}^{\prime}, g_{a b}^{\prime}} \longrightarrow\left(\alpha_{a} \times \beta_{a}\right) \circ \Pi_{V_{a}^{\prime}}\right|_{Y_{a b}^{\prime}} ^{*}\left(\mathcal{P} \mathcal{V}_{U \times \mathbb{C}^{n}, f \boxplus z_{1}^{2}+\cdots+z_{n}^{2}}^{\bullet}\right)
\end{gathered}
$$

in $\operatorname{Perv}\left(Y_{a b}^{\prime}\right)$, where $\operatorname{det}[\cdots]$ maps $Y_{a b}^{\prime \text { red }} \rightarrow\{ \pm 1\}$.

Consider the morphisms

$$
\begin{gathered}
\left.\Pi_{U_{a}^{\prime}}\right|_{X_{a b}^{\prime}} ^{*}\left(\delta_{a}\right),\left.\Pi_{U_{b}^{\prime}}\right|_{X_{a b}^{\prime}} ^{*}\left(\delta_{b}\right):\left.\left.\left(\Phi \circ \iota_{a} \circ \Pi_{U_{a}^{\prime}}\right)\right|_{X_{a b}^{\prime}} ^{*}\left(\mathcal{P} V_{V, g}^{\bullet}\right) \otimes_{\mathbb{Z} / 2 \mathbb{Z}}\left(\iota_{a} \circ \Pi_{U_{a}^{\prime}}\right)\right|_{X_{a b}^{\prime}} ^{*}\left(P_{\Phi}\right) \\
\left.\longrightarrow\left(\Phi \circ \iota_{a} \circ \Pi_{U_{a}^{\prime}}\right)\right|_{X_{a b}^{\prime}} ^{*}\left(\mathcal{P} \mathcal{V}_{V, g}^{\bullet}\right) .
\end{gathered}
$$

As in (B) above, these are defined using two different trivializations of the principal $\mathbb{Z} / 2 \mathbb{Z}$-bundle $\left.\left(\iota_{a} \circ \Pi_{U_{a}^{\prime}}\right)\right|_{X_{a b}^{\prime}} ^{*}\left(P_{\Phi}\right) \rightarrow X_{a b}^{\prime}$, defined using

$$
\left.\Pi_{U_{a}^{\prime}}\right|_{X_{a b}^{\prime r e d}} ^{*}\left(\hat{\beta}_{a}\right),\left.\Pi_{U_{b}^{\prime}}\right|_{X_{a b}^{\prime r e d}} ^{*}\left(\hat{\beta}_{b}\right):\left.\left\langle\mathrm{d} z_{1}, \ldots, \mathrm{d} z_{n}\right\rangle_{X_{a b}^{\prime r e d}} \longrightarrow\left(\iota_{a} \circ \Pi_{U_{a}^{\prime}}\right)\right|_{X_{a b}^{\prime r e d}} ^{*}\left(N_{U V}^{*}\right),
$$

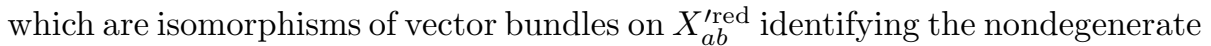
quadratic forms $\sum_{j=1}^{n} \mathrm{~d} z_{j}^{2}$ on $\left\langle\mathrm{d} z_{1}, \ldots, \mathrm{d} z_{n}\right\rangle_{X_{a b}^{\prime r e d}}$ and $\left.\left(\iota_{a} \circ \Pi_{U_{a}^{\prime}}\right)\right|_{X_{a b}^{\prime r e d}} ^{*}\left(q_{U V}\right)$ on $\left.\left(\iota_{a} \circ \Pi_{U_{a}^{\prime}}\right)\right|_{X_{a b}^{\prime r e d}} ^{*}\left(N_{U V}^{*}\right)$, for $\hat{\beta}_{a}, \hat{\beta}_{b}$ as in (5.3). Thus we see that

$$
\left.\Pi_{U_{a}^{\prime}}\right|_{X_{a b}^{\prime}} ^{*}\left(\delta_{a}\right)=\left.\operatorname{det}\left[\left.\left.\Pi_{U_{a}^{\prime}}\right|_{X_{a b}^{\prime r e d}} ^{*}\left(\hat{\beta}_{a}\right) \circ \Pi_{U_{b}^{\prime}}\right|_{X_{a b}^{\prime r e d}} ^{*}\left(\hat{\beta}_{b}\right)^{-1}\right] \cdot \Pi_{U_{b}^{\prime}}\right|_{X_{a b}^{\prime}} ^{*}\left(\delta_{b}\right),
$$

where $\operatorname{det}[\cdots]$ maps $X_{a b}^{\prime \text { red }} \rightarrow\{ \pm 1\}$ since both isomorphisms in (5.22) identify the same nondegenerate quadratic forms.

We have an exact sequence of vector bundles on $X_{a b}^{\text {red }}$ :

$$
\left.\left.\left.0 \longrightarrow T U_{a b}^{\prime}\right|_{X_{a b}^{\prime r e d}} \longrightarrow \Phi_{a b}^{\prime}\right|_{X_{a b}^{\prime r e d}} ^{*}\left(T V_{a b}^{\prime}\right) \longrightarrow\left(\iota_{a} \circ \Pi_{U_{a}^{\prime}}\right)\right|_{X_{a b}^{\prime r e d}} ^{*}\left(N_{U V}\right) \longrightarrow 0 .
$$

Choosing a local splitting of this sequence, we may identify

$$
\begin{aligned}
& \left.\Phi_{a b}^{\prime}\right|_{X_{a b}^{\prime r \text { red }}} ^{*}\left[\left.\left.\mathrm{~d}\left(\left(\alpha_{b} \times \beta_{b}\right) \circ \Pi_{V_{b}^{\prime}}\right)\right|_{Y_{a b}^{\prime r \text { red }}} ^{-1} \circ \mathrm{d}\left(\left(\alpha_{a} \times \beta_{a}\right) \circ \Pi_{V_{a}^{\prime}}\right)\right|_{Y_{a b}^{\prime r e d}}\right] \\
& \cong\left(\begin{array}{cc}
\operatorname{id}_{\left.T U_{a b}^{\prime}\right|_{X_{a b}^{\prime r e d}}} & * \\
0 & \left(\left.\left.\Pi_{U_{a}^{\prime}}\right|_{X_{a b}^{\text {red }}} ^{* \text { red }}\left(\hat{\beta}_{a}\right) \circ \Pi_{U_{b}^{\prime}}\right|_{X_{a b}^{\prime r \text { red }}} ^{*}\left(\hat{\beta}_{b}\right)^{-1}\right)^{*}
\end{array}\right) .
\end{aligned}
$$


Therefore

$$
\begin{gathered}
\left.\Phi_{a b}^{\prime}\right|_{X_{a b}^{\prime r e d}} ^{*}\left(\operatorname{det}\left[\left.\left.\mathrm{d}\left(\left(\alpha_{b} \times \beta_{b}\right) \circ \Pi_{V_{b}^{\prime}}\right)\right|_{Y_{a b}^{\prime \text { red }}} ^{-1} \circ \mathrm{d}\left(\left(\alpha_{a} \times \beta_{a}\right) \circ \Pi_{V_{a}^{\prime}}\right)\right|_{Y_{a b}^{\prime \text { red }}}\right]\right) \\
=\operatorname{det}\left[\left.\left.\Pi_{U_{a}^{\prime}}\right|_{X_{a b}^{\prime r \text { red }}} ^{*}\left(\hat{\beta}_{a}\right) \circ \Pi_{U_{b}^{\prime}}\right|_{X_{a b}^{\prime r e d}} ^{*}\left(\hat{\beta}_{b}\right)^{-1}\right]: X_{a b}^{\prime \text { red }} \longrightarrow\{ \pm 1\} .
\end{gathered}
$$

Now

$$
\begin{aligned}
& \left.\Pi_{U_{a}^{\prime}}\right|_{X_{a b}^{\prime}} ^{*}\left(\Theta_{a}\right)=\left.\left.\left.\Pi_{U_{a}^{\prime}}\right|_{X_{a b}^{\prime}} ^{*}\left(\delta_{a}^{-1}\right) \circ\left(\Phi_{a}^{\prime} \circ \Pi_{U_{a}^{\prime}}\right)\right|_{X_{a b}^{\prime}} ^{*}\left(\mathcal{P} \mathcal{V}_{\jmath_{a}}\right) \circ\left(\Phi_{a}^{\prime} \circ \Pi_{U_{a}^{\prime}}\right)\right|_{X_{a b}^{\prime}} ^{*}\left(\mathcal{P} \mathcal{V}_{\alpha_{a} \times \beta_{a}}^{-1}\right) \\
& \left.\left.\circ\left(\left(\operatorname{id}_{X} \times 0\right) \circ \iota_{a} \circ \Pi_{U_{a}^{\prime}}\right)\right|_{X_{a b}^{\prime}} ^{*}\left(\mathcal{T} \mathcal{S}_{U, f, \mathbb{C}^{n}, \Sigma_{j} z_{j}^{2}}^{-1}\right) \circ\left(\iota_{a} \circ \Pi_{U_{a}^{\prime}}\right)\right|_{X_{a b}^{\prime}} ^{*}(\gamma) \\
& =\left.\left.\left.\left.\Pi_{U_{a}^{\prime}}\right|_{X_{a b}^{\prime}} ^{*}\left(\delta_{a}^{-1}\right) \circ\left(\Pi_{V_{a}^{\prime}} \circ \Phi_{a b}^{\prime}\right)\right|_{X_{a b}^{\prime}} ^{*}\left(\mathcal{P} \mathcal{V}_{J_{a}}\right) \circ \Phi_{a b}^{\prime}\right|_{X_{a b}^{\prime}} ^{*}\left(\mathcal{P} \mathcal{V}_{\Pi_{V_{a}^{\prime}}}\right) \circ \Phi_{a b}^{\prime}\right|_{X_{a b}^{\prime}} ^{*}\left(\mathcal{P} \mathcal{V}_{\Pi_{V_{a}^{\prime}}}^{-1}\right) \\
& \left.\left.\left.\circ\left(\Pi_{V_{a}^{\prime}} \circ \Phi_{a b}^{\prime}\right)\right|_{X_{a b}^{\prime}} ^{*}\left(\mathcal{P} \mathcal{V}_{\alpha_{a} \times \beta_{a}}^{-1}\right) \circ\left(\left(\mathrm{id}_{X} \times 0\right) \circ \iota_{a} \circ \Pi_{U_{a}^{\prime}}\right)\right|_{X_{a b}^{\prime}} ^{*}\left(\mathcal{T S}_{U, f, \mathbb{C}^{n}, \Sigma_{j} z_{j}^{2}}^{-1}\right) \circ\left(\iota_{a} \circ \Pi_{U_{a}^{\prime}}\right)\right|_{X_{a b}^{\prime}} ^{*}(\gamma) \\
& =\left.\left.\left.\Pi_{U_{a}^{\prime}}\right|_{X_{a b}^{\prime}} ^{*}\left(\delta_{a}^{-1}\right) \circ \Phi_{a b}^{\prime}\right|_{X_{a b}^{\prime}} ^{*}\left(\mathcal{P} \mathcal{V}_{\jmath_{a} \circ \Pi_{V_{a}^{\prime}}}\right) \circ \Phi_{a b}^{\prime}\right|_{X_{a b}^{\prime}} ^{*}\left(\mathcal{P} \mathcal{V}_{\left(\alpha_{a} \times \beta_{a}\right) \circ \Pi_{V_{a}^{\prime}}}^{-1}\right) \\
& \left.\left.\circ\left(\left(\operatorname{id}_{X} \times 0\right) \circ \iota_{a} \circ \Pi_{U_{a}^{\prime}}\right)\right|_{X_{a b}^{\prime}} ^{*}\left(\mathcal{T} \mathcal{S}_{U, f, \mathbb{C}^{n}, \Sigma_{j} z_{j}^{2}}^{-1}\right) \circ\left(\iota_{a} \circ \Pi_{U_{a}^{\prime}}\right)\right|_{X_{a b}^{\prime}} ^{*}(\gamma) \\
& =\operatorname{det}\left[\left.\left.\Pi_{U_{a}^{\prime}}\right|_{X_{a b}^{\prime r e d}} ^{*}\left(\hat{\beta}_{a}\right) \circ \Pi_{U_{b}^{\prime}}\right|_{X_{a b}^{\prime r e d}} ^{*}\left(\hat{\beta}_{b}\right)^{-1}\right]^{-1} \text {. } \\
& \left.\Phi_{a b}^{\prime}\right|_{X_{a b}^{\prime r e d}} ^{*}\left(\operatorname{det}\left[\left.\left.\mathrm{d}\left(\left(\alpha_{b} \times \beta_{b}\right) \circ \Pi_{V_{b}^{\prime}}\right)\right|_{Y_{a b}^{\prime \text { red }}} ^{-1} \circ \mathrm{d}\left(\left(\alpha_{a} \times \beta_{a}\right) \circ \Pi_{V_{a}^{\prime}}\right)\right|_{Y_{a b}^{\prime r e d}}\right]\right) \text {. } \\
& \left.\left.\left.\Pi_{U_{b}^{\prime}}\right|_{X_{a b}^{\prime}} ^{*}\left(\delta_{b}^{-1}\right) \circ \Phi_{a b}^{\prime}\right|_{X_{a b}^{\prime}} ^{*}\left(\mathcal{P} \mathcal{V}_{\jmath_{b}} \circ \Pi_{V_{b}^{\prime}}\right) \circ \Phi_{a b}^{\prime}\right|_{X_{a b}^{\prime}} ^{*}\left(\mathcal{P} \mathcal{V}_{\left(\alpha_{b} \times \beta_{b}\right) \circ \Pi_{V_{b}^{\prime}}}^{-1}\right) \\
& \left.\left.\circ\left(\left(\operatorname{id}_{X} \times 0\right) \circ \iota_{b} \circ \Pi_{U_{b}^{\prime}}\right)\right|_{X_{a b}^{\prime}} ^{*}\left(\mathcal{T} \mathcal{S}_{U, f, \mathbb{C}^{n}, \Sigma_{j} z_{j}^{2}}^{-1}\right) \circ\left(\iota_{b} \circ \Pi_{U_{b}^{\prime}}\right)\right|_{X_{a b}^{\prime}} ^{*}(\gamma) \\
& =\left.\left.\left.\left.\Pi_{U_{b}^{\prime}}\right|_{X_{a b}^{\prime}} ^{*}\left(\delta_{b}^{-1}\right) \circ\left(\Pi_{V_{b}^{\prime}} \circ \Phi_{a b}^{\prime}\right)\right|_{X_{a b}^{\prime}} ^{*}\left(\mathcal{P} \mathcal{V}_{J_{b}}\right) \circ \Phi_{a b}^{\prime}\right|_{X_{a b}^{\prime}} ^{*}\left(\mathcal{P} \mathcal{V}_{\Pi_{V_{b}^{\prime}}}\right) \circ \Phi_{a b}^{\prime}\right|_{X_{a b}^{\prime}} ^{*}\left(\mathcal{P} \mathcal{V}_{\Pi_{V_{b}^{\prime}}}^{-1}\right) \\
& \left.\left.\left.\circ\left(\Pi_{V_{b}^{\prime}} \circ \Phi_{a b}^{\prime}\right)\right|_{X_{a b}^{\prime}} ^{*}\left(\mathcal{P} \mathcal{V}_{\alpha_{b} \times \beta_{b}}^{-1}\right) \circ\left(\left(\operatorname{id}_{X} \times 0\right) \circ \iota_{b} \circ \Pi_{U_{b}^{\prime}}\right)\right|_{X_{a b}^{\prime}} ^{*}\left(\mathcal{T S}_{U, f, \mathbb{C}^{n}, \Sigma_{j} z_{j}^{2}}^{-1}\right) \circ\left(\iota_{b} \circ \Pi_{U_{b}^{\prime}}\right)\right|_{X_{a b}^{\prime}} ^{*}(\gamma) \\
& =\left.\left.\left.\Pi_{U_{b}^{\prime}}\right|_{X_{a b}^{\prime}} ^{*}\left(\delta_{b}^{-1}\right) \circ\left(\Phi_{b}^{\prime} \circ \Pi_{U_{b}^{\prime}}\right)\right|_{X_{a b}^{\prime}} ^{*}\left(\mathcal{P} \mathcal{V}_{J_{b}}\right) \circ\left(\Phi_{b}^{\prime} \circ \Pi_{U_{b}^{\prime}}\right)\right|_{X_{a b}^{\prime}} ^{*}\left(\mathcal{P} \mathcal{V}_{\alpha_{b} \times \beta_{b}}^{-1}\right) \\
& \left.\left.\circ\left(\left(\operatorname{id}_{X} \times 0\right) \circ \iota_{b} \circ \Pi_{U_{b}^{\prime}}\right)\right|_{X_{a b}^{\prime}} ^{*}\left(\mathcal{T} \mathcal{S}_{U, f, \mathbb{C}^{n}, \Sigma_{j} z_{j}^{2}}^{-1}\right) \circ\left(\iota_{b} \circ \Pi_{U_{b}^{\prime}}\right)\right|_{X_{a b}^{\prime}} ^{*}(\gamma)=\left.\Pi_{U_{b}^{\prime}}\right|_{X_{a b}^{\prime}} ^{*}\left(\Theta_{b}\right) \text {, }
\end{aligned}
$$

using (5.18) in the first and seventh steps, (5.19) in the second and sixth, (2.18) in the third, (5.21), (5.23), $\iota_{a} \circ \Pi_{U_{a}^{\prime}}=\iota_{b} \circ \Pi_{U_{b}^{\prime}}$ and $\jmath_{a} \circ \Pi_{V_{a}^{\prime}}=\jmath_{b} \circ \Pi_{V_{b}^{\prime}}$ in the fourth, and (2.18) and (5.24) in the fifth. Therefore Theorem 2.7(i) applied to the étale open cover $\left\{\left.\iota_{a}\right|_{X_{a}^{\prime}}: X_{a}^{\prime} \rightarrow X\right\}_{a \in A}$ of $X$ shows that there is a unique isomorphism $\Theta_{\Phi}$ in (5.13) with $\left.\iota_{a}\right|_{X_{a}^{\prime}} ^{*}\left(\Theta_{\Phi}\right)=\Theta_{a}$ for all $a \in A$.

Suppose $\left\{\left(U_{a}^{\prime}, \ldots, Y_{a}^{\prime}\right): a \in A\right\}$ and $\left\{\left(\tilde{U}_{a}^{\prime}, \ldots, \tilde{Y}_{a}^{\prime}\right): a \in \tilde{A}\right\}$ are alternative choices above, yielding morphisms $\Theta_{\Phi}$ and $\tilde{\Theta}_{\Phi}$ in (5.13). By running the same construction using the family $\left\{\left(U_{a}^{\prime}, \ldots, Y_{a}^{\prime}\right): a \in A\right\} \amalg\left\{\left(\tilde{U}_{a}^{\prime}, \ldots, \tilde{Y}_{a}^{\prime}\right): a \in \tilde{A}\right\}$, we can show that $\Theta_{\Phi}=\tilde{\Theta}_{\Phi}$, so $\Theta_{\Phi}$ is independent of the choice of $\left\{\left(U_{a}^{\prime}, \ldots, Y_{a}^{\prime}\right)\right.$ : $a \in A\}$ above. Let $U^{\prime}, V^{\prime}, \iota, \jmath, \Phi^{\prime}, \alpha, \beta, X^{\prime}, Y^{\prime}, f^{\prime}, g^{\prime}$ be as in Theorem 5.1(i). Constructing $\Theta_{\Phi}$ using $\left\{\left(U_{a}^{\prime}, \ldots, Y_{a}^{\prime}\right): a \in A\right\} \amalg\left\{\left(U^{\prime}, \ldots, Y^{\prime}\right)\right\}$, we see from (5.18) that (5.17) commutes. This completes the construction of $\Theta_{\Phi}$.

To see that (5.14)-(5.15) commute, in the situation of (5.17) we show that Verdier duality and monodromy operators commute with each morphism in (5.17). Going clockwise from the top left corner, $\left.\iota\right|_{X^{\prime}} ^{*}(\gamma)$ is compatible with 
Verdier duality and monodromy because of the commutative diagrams
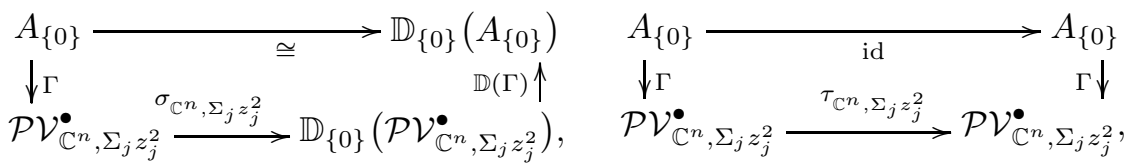

where $\Gamma: A_{\{0\}} \rightarrow \mathcal{P} \mathcal{V}_{\mathbb{C}^{n}, \Sigma_{j} z_{j}^{2}}$ is the isomorphism used to define $\gamma$ in (A) above. Equations (2.9)-(2.10) imply that $\iota_{X^{\prime}}^{*} \circ\left(\operatorname{id}_{X} \times 0\right)^{*}\left(\mathcal{T} \mathcal{S}_{U, f, \mathbb{C}^{n}, \Sigma_{j} z_{j}^{2}}^{-1}\right)$ is compatible with Verdier duality and monodromy, and (2.16)-(2.17) imply that $\left.\Phi^{\prime}\right|_{X^{\prime}} ^{*}\left(\mathcal{P} \mathcal{V}_{\jmath}\right),\left.\Phi^{\prime}\right|_{X^{\prime}} ^{*}\left(\mathcal{P} \mathcal{V}_{\alpha \times \beta}^{-1}\right)$ are. Also $\delta$ is compatible with Verdier duality and monodromy, since these do not affect the trivialization of $\left.\iota\right|_{X^{\prime}} ^{*}\left(P_{\Phi}\right) \rightarrow X^{\prime}$ used to define $\delta$ in (B) above.

Thus by (5.17) we see that $\left.\iota\right|_{X^{\prime}} ^{*}\left(\Theta_{\Phi}\right)$ is compatible with Verdier duality and monodromy, that is, $\iota_{X^{\prime}}^{*}$ applied to (5.14) an étale open cover of $X$ by such $\left.\iota\right|_{X^{\prime}}: X^{\prime} \rightarrow X$, Theorem 2.7(i) implies that (5.14) - (5.15) commute.

Finally, if $U=V, f=g$ and $\Phi=\operatorname{id}_{U}$ then $J_{\Phi}=\mathrm{id}:\left.\left.K_{U}^{2}\right|_{X^{\text {red }}} \rightarrow K_{U}^{2}\right|_{X^{\text {red }}}$ in (5.9), which has a natural square root $\alpha=$ id $:\left.\left.K_{U}\right|_{X^{\text {red }}} \rightarrow K_{U}\right|_{X^{\text {red }}}$, so $\pi_{\Phi}: P_{\Phi} \rightarrow X$ is trivial in Definition [5.2. In (5.17) we may put $U^{\prime}=V^{\prime}=U$, $\iota=\jmath=\alpha=\operatorname{id}_{U}, n=0, \beta=0, X^{\prime}=Y^{\prime}=X, f^{\prime}=g^{\prime}=f$, and then each morphism in (5.17) is essentially the identity on $\mathcal{P V}_{U, f}^{\bullet}$, so $\Theta_{\Phi}=\operatorname{id}_{X}^{*}\left(\Theta_{\Phi}\right)=$ $\operatorname{id}_{\mathcal{P} \mathcal{V}_{U, f}}$. This proves Theorem 5.4(a).

\subsection{Theorem 5.4(b): $\Theta_{\Phi}$ depends only on $\left.\Phi\right|_{X}: X \rightarrow Y$}

Suppose $\Phi, \tilde{\Phi}: U \rightarrow V$ are alternative choices in Theorem 5.4(a) with $\left.\Phi\right|_{X}=$ $\left.\tilde{\Phi}\right|_{X}: X \rightarrow Y$, so that $P_{\Phi}=P_{\tilde{\Phi}}$ by Lemma [5.3. Fix $x \in X$, let $a \neq b$ be labels, and let $U_{a}^{\prime}, V_{a}^{\prime}, \iota_{a}, \jmath_{a}, \Phi_{a}^{\prime}, \alpha_{a}, \beta_{a}, X_{a}^{\prime}, Y_{a}^{\prime}, f_{a}^{\prime}, g_{a}^{\prime}$ be as in Theorem [5.1(i) for $x, \Phi$ and $U_{b}^{\prime}, V_{b}^{\prime}, \ldots, g_{b}^{\prime}$ as in Theorem 5.1 (i) for $x, \tilde{\Phi}$. As in 55.1, define $\Theta_{a}, \Theta_{b}$ and $U_{a b}^{\prime}, V_{a b}^{\prime}, \Pi_{U_{a}^{\prime}}, \Pi_{U_{b}^{\prime}}, \Pi_{V_{a}^{\prime}}, \Pi_{V_{b}^{\prime}}, \Phi_{a b}^{\prime}, f_{a b}^{\prime}, g_{a b}^{\prime}, X_{a b}^{\prime}, Y_{a b}^{\prime}$, and follow the proof in $\$ 5.1$ from (5.19) as far as (5.25).

This proof does not actually need $U_{a}^{\prime}, \ldots, g_{a}, \Theta_{a}$ and $U_{b}^{\prime}, \ldots, g_{b}, \Theta_{b}$ to be defined using the same $\Phi: U \rightarrow V$, it only uses in (5.20)-(5.22) that $\left.\Phi\right|_{X}$ : $X \rightarrow Y$ is the same for $U_{a}^{\prime}, \ldots, \Theta_{a}$ and $U_{b}^{\prime}, \ldots, \Theta_{b}$. Thus we can apply it with $U_{a}^{\prime}, \ldots, \Theta_{a}$ defined using $\Phi$, and $U_{b}^{\prime}, \ldots, \Theta_{b}$ defined using $\tilde{\Phi}$. Hence

$$
\begin{aligned}
\left.\left(\iota_{a} \circ \Pi_{U_{a}^{\prime}}\right)\right|_{X_{a b}^{\prime}} ^{*}\left(\Theta_{\Phi}\right) & =\left.\Pi_{U_{a}^{\prime}}\right|_{X_{a b}^{\prime}} ^{*}\left(\Theta_{a}\right)=\left.\Pi_{U_{b}^{\prime}}\right|_{X_{a b}^{\prime}} ^{*}\left(\Theta_{b}\right) \\
& =\left.\left(\iota_{b} \circ \Pi_{U_{b}^{\prime}}\right)\right|_{X_{a b}^{\prime}} ^{*}\left(\Theta_{\tilde{\Phi}}\right)=\left.\left(\iota_{a} \circ \Pi_{U_{a}^{\prime}}\right)\right|_{X_{a b}^{\prime}} ^{*}\left(\Theta_{\tilde{\Phi}}\right),
\end{aligned}
$$

using $\left.\iota_{a}\right|_{X_{a}^{\prime}} ^{*}\left(\Theta_{\Phi}\right)=\Theta_{a}$ in the first step, (5.25) in the second, $\left.\iota_{b}\right|_{X_{b}^{\prime}} ^{*}\left(\Theta_{\tilde{\Phi}}\right)=\Theta_{b}$ in the third, and $\iota_{a} \circ \Pi_{U_{a}^{\prime}}=\iota_{b} \circ \Pi_{U_{b}^{\prime}}$ in the fourth. As such $\left.\iota_{a} \circ \Pi_{U_{a}^{\prime}}\right|_{X_{a b}^{\prime}}: X_{a b}^{\prime} \rightarrow X$ form an étale open cover of $X$, this implies that $\Theta_{\Phi}=\Theta_{\tilde{\Phi}}$ by Theorem [2.7(i). 


\subsection{Theorem 5.4(c): composition of the $\Theta_{\Phi}$}

Let $U, V, W, f, g, h, X, Y, Z, \Phi, \Psi$ be as in Theorem 5.4(c). Let $x \in X$, and set $y=\Phi(x) \in Y$. Apply Theorem 5.1(i) to $U, V, f, g, X, Y, \Phi, x$ to get $\mathbb{C}$-schemes $U^{\prime}, V^{\prime}$, a point $x^{\prime} \in U^{\prime}$, morphisms $\iota: U^{\prime} \rightarrow U, \jmath: V^{\prime} \rightarrow V, \Phi^{\prime}: U^{\prime} \rightarrow V^{\prime}$, $\alpha: V^{\prime} \rightarrow U$ and $\beta: V^{\prime} \rightarrow \mathbb{C}^{m}$ where $m=\operatorname{dim} V-\operatorname{dim} U$, and $f^{\prime}:=f \circ \iota: U^{\prime} \rightarrow$ $\mathbb{C}, g^{\prime}:=g \circ \jmath: V^{\prime} \rightarrow \mathbb{C}, X^{\prime}:=\operatorname{Crit}\left(f^{\prime}\right) \subseteq U^{\prime}, Y^{\prime}:=\operatorname{Crit}\left(g^{\prime}\right) \subseteq V^{\prime}$, satisfying conditions including $\iota, \jmath, \alpha \times \beta$ étale, (5.1) commutes, and $\iota\left(x^{\prime}\right)=x$.

Similarly, apply Theorem [5.1(i) to $V, W, g, h, Y, Z, \Psi, y$ to get $\mathbb{C}$-schemes $\tilde{V}, \tilde{W}$, a point $\tilde{y} \in \tilde{V}$, morphisms $\tilde{\iota}: \tilde{V} \rightarrow V, \tilde{\jmath}: \tilde{W} \rightarrow W, \tilde{\Psi}: \tilde{V} \rightarrow \tilde{W}$, $\tilde{\alpha}: \tilde{W} \rightarrow V$ and $\tilde{\beta}: \tilde{W} \rightarrow \mathbb{C}^{n}$ where $n=\operatorname{dim} W-\operatorname{dim} V$, and $\tilde{g}:=g \circ \tilde{\iota}: \tilde{V} \rightarrow \mathbb{C}$, $\tilde{h}:=h \circ \tilde{\jmath}: \tilde{W} \rightarrow \mathbb{C}, \tilde{Y}:=\operatorname{Crit}(\tilde{g}) \subseteq \tilde{V}, \tilde{Z}:=\operatorname{Crit}(\tilde{h}) \subseteq \tilde{W}$, satisfying conditions.

Define $\hat{U}=U^{\prime} \times_{\Phi \circ \iota, V, \tilde{\imath}} \tilde{V}$ and $\hat{W}=V^{\prime} \times_{\jmath, V, \tilde{\alpha}} \tilde{W}$, with projections $\Pi_{U^{\prime}}: \hat{U} \rightarrow$ $U^{\prime}, \Pi_{\tilde{V}}: \hat{U} \rightarrow \tilde{V}, \Pi_{V^{\prime}}: \hat{W} \rightarrow V^{\prime}, \Pi_{\tilde{W}}: \hat{W} \rightarrow \tilde{W}$. As $x^{\prime} \in U^{\prime}$ and $\tilde{y} \in \tilde{V}$ with $\Phi \circ \iota\left(x^{\prime}\right)=y=\tilde{\iota}(\tilde{y})$, there exists $\hat{x} \in \hat{U}$ with $\Pi_{U^{\prime}}(\hat{x})=x^{\prime}$ and $\Pi_{\tilde{V}}(\hat{x})=\tilde{y}$. Set $\hat{f}:=f^{\prime} \circ \Pi_{U^{\prime}}: \hat{U} \rightarrow \mathbb{C}$ and $\hat{h}:=\tilde{h} \circ \Pi_{\tilde{W}}: \hat{W} \rightarrow \mathbb{C}$, and $\hat{X}:=\operatorname{Crit}(\hat{f}) \subseteq \hat{U}$, $\hat{Z}:=\operatorname{Crit}(\hat{h}) \subseteq \hat{W}$. The morphisms $\Phi^{\prime} \circ \Pi_{U^{\prime}}: \hat{U} \rightarrow V^{\prime}, \tilde{\Psi} \circ \Pi_{\tilde{V}}: \hat{U} \rightarrow \tilde{W}$ satisfy

$$
\jmath \circ\left(\Phi^{\prime} \circ \Pi_{U^{\prime}}\right)=\Phi \circ \iota \circ \Pi_{U^{\prime}}=\tilde{\iota} \circ \Pi_{\tilde{V}}=\tilde{\alpha} \circ\left(\tilde{\Psi} \circ \Pi_{\tilde{V}}\right) .
$$

Hence there exists a unique morphism $\widehat{\Psi \circ \Phi} \Phi: \hat{U} \rightarrow \hat{W}$ such that $\Pi_{V^{\prime}} \circ$ $\widehat{\Psi \circ \Phi}=\Phi^{\prime} \circ \Pi_{U^{\prime}}$ and $\Pi_{\tilde{W}} \circ \Psi \circ \Phi=\tilde{\Psi} \circ \Pi_{\tilde{V}}$. Then the following diagram

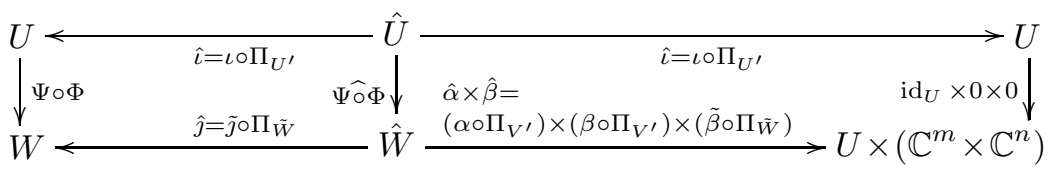

is the analogue of (5.1) for $U, W, f, h, X, Z, \Psi \circ \Phi, x$, and the conclusions of Theorem 5.1(i) hold. Thus (5.17) holds for $\Theta_{\Phi}$ using $U^{\prime}, V^{\prime}, X^{\prime}, Y^{\prime} \iota, \jmath, \Phi^{\prime}, \alpha, \beta, m$, and for $\Theta_{\Psi}$ using $\tilde{V}, \tilde{W}, \tilde{Y}, \tilde{Z}, \tilde{\iota}, \tilde{\jmath}, \tilde{\Psi}, \tilde{\alpha}, \tilde{\beta}, n$, and for $\Theta_{\Psi \circ \Phi}$ using $\tilde{U}, \hat{W}, \hat{X}, \hat{Z}, \hat{\iota}$, $\hat{\jmath}, \Psi \circ \Phi, \hat{\alpha}, \hat{\beta}, m+n$. 
We have a commutative diagram in $\operatorname{Perv}(\hat{X})$ :

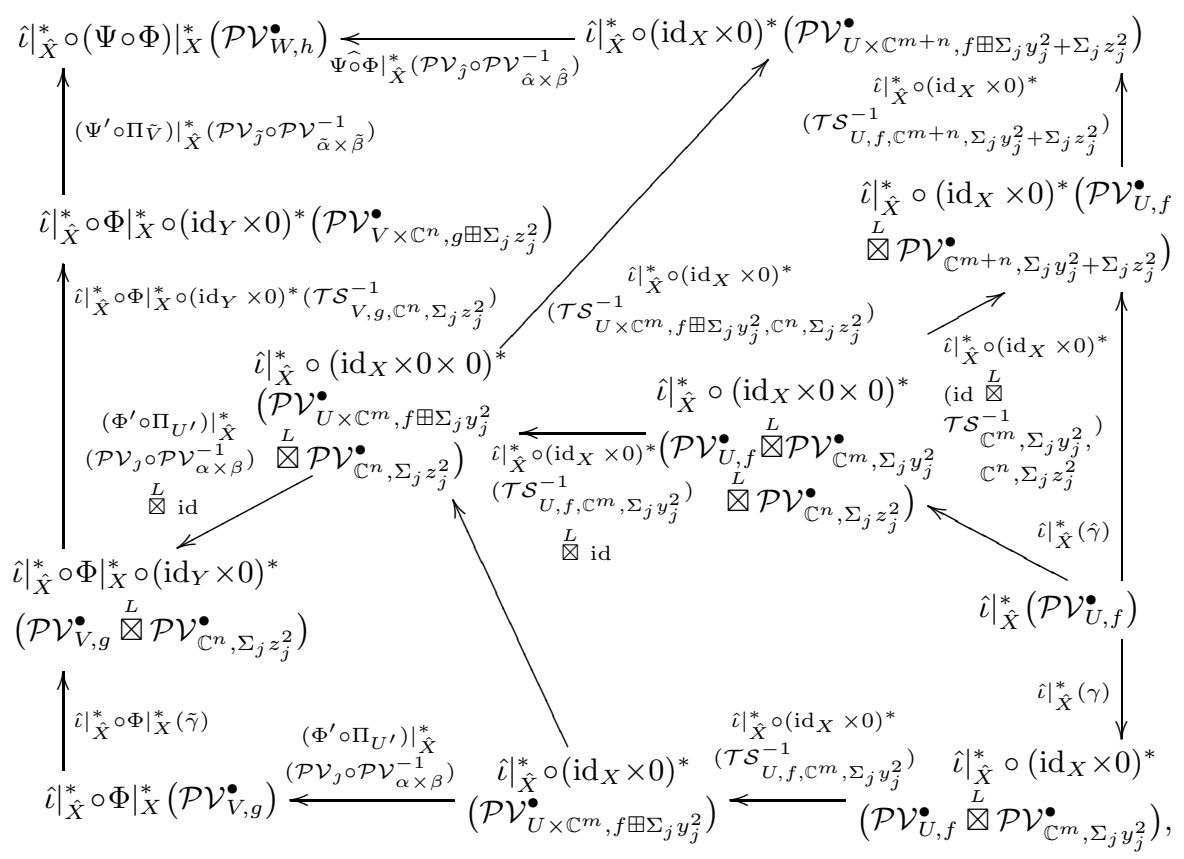

where the top right quadrilateral commutes because of associativity in the Thom-Sebastiani Theorem for $\mathcal{P} \mathcal{V}_{V, f}^{\bullet}$, Theorem 2.13 Also we have

$$
\begin{aligned}
& \left.\left.\left.(\Psi \circ \Phi \circ \hat{\imath})\right|_{\hat{X}} ^{*}\left(\mathcal{P} \mathcal{V}_{W, h}^{\bullet}\right) \longleftarrow \hat{\delta}_{\hat{\delta}}(\Psi \circ \Phi \circ \hat{\imath})\right|_{\hat{X}} ^{*}\left(\mathcal{P} \mathcal{V}_{W, h}^{\bullet}\right) \otimes_{\mathbb{Z} / 2 \mathbb{Z}} \hat{\imath}\right|_{\hat{X}} ^{*}\left(P_{\Psi \circ \Phi}\right) \\
& \uparrow \Pi_{\tilde{V}} l_{\hat{X}}^{*}(\tilde{\delta}) \quad \operatorname{id} \otimes \hat{\imath}_{\hat{X}}^{*}\left(\Xi_{\Psi, \Phi)} \downarrow\right. \\
& \left.\left.(\Psi \circ \Phi \circ \hat{\imath})\right|_{\hat{X}} ^{*}\left(\mathcal{P} \mathcal{V}_{W, h}^{\bullet}\right) \quad(\Psi \circ \Phi \circ \hat{\imath})\right|_{\hat{X}} ^{*}\left(\mathcal{P} \mathcal{V}_{W, h}^{\bullet}\right) \\
& \left.\left.\left.\otimes_{\mathbb{Z} / 2 \mathbb{Z}}\left(\tilde{\iota} \circ \Pi_{\tilde{V}}\right)\right|_{\hat{X}} ^{*}\left(P_{\Psi}\right) \quad \otimes_{\mathbb{Z} / 2 \mathbb{Z}}\left(\tilde{\iota} \circ \prod_{\tilde{V}}\right)\right|_{\hat{X}} ^{*}\left(P_{\Psi}\right) \otimes_{\mathbb{Z} / 2 \mathbb{Z}} \hat{\iota}\right|_{\hat{X}} ^{*}\left(P_{\Phi}\right) \\
& \left.\left.\uparrow(\Phi \circ \hat{\imath})\right|_{\hat{X}} ^{*}\left(\Theta_{\Psi}\right) \quad(\Phi \circ \hat{\imath})\right|_{\hat{X}} ^{*}\left(\Theta_{\Psi}^{-1}\right) \otimes \mathrm{id}
\end{aligned}
$$

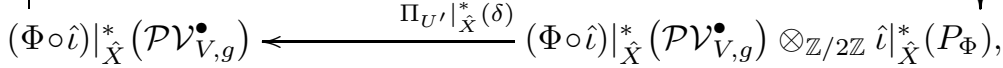

which commutes because the trivializations of $\left.\iota\right|_{X^{\prime}}\left(P_{\Phi}\right),\left.\tilde{\iota}\right|_{\tilde{X}}\left(P_{\Psi}\right),\left.\hat{\iota}\right|_{\hat{X}}\left(P_{\Psi \circ \Phi}\right)$ used to define $\delta, \tilde{\delta}, \hat{\delta}$ are compatible with $\Xi_{\Psi, \Phi}$.

Combining (5.26) and (5.27) with $\left.\Pi_{U^{\prime}}\right|_{\hat{X}} ^{*}$ applied to (5.17) for $\Theta_{\Phi}$, and $\left.\Pi_{\tilde{V}}\right|_{\hat{X}} ^{*}$ applied to (5.17) for $\Theta_{\Psi}$, and (5.17) for $\Theta_{\Psi \circ \Phi}$, we can show that the following 
diagram commutes in $\operatorname{Perv}(\hat{X})$ :

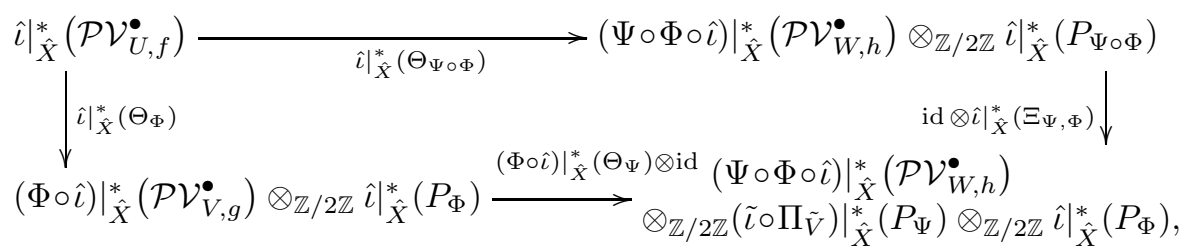

which is $\left.\hat{\imath}\right|_{\hat{X}} ^{*}$ applied to (5.16). Since such $\left.\hat{\imath}\right|_{\hat{X}}: \hat{X} \rightarrow X$ form an étale cover of $X$, equation (5.16) commutes by Theorem 2.7(i). This proves Theorem 5.4(c).

\section{4 $\mathscr{D}$-modules and mixed Hodge modules}

By Theorem 5.1(iv),(v), the earlier parts of that result hold for our other contexts in 2.6 2.10. Once again, the proofs of Theorem 5.4(a)-(c) then carry over to the other contexts using the general framework of 2.5 , now also making use of property (vii).

\section{Perverse sheaves on oriented d-critical loci}

\subsection{Background material on d-critical loci}

Here are some of the main definitions and results on d-critical loci, from Joyce [23. Th.s 2.1, 2.20, 2.28 \& Def.s 2.5, 2.18, 2.31]. For the algebraic case we work with $\mathbb{C}$-schemes.

Theorem 6.1. Let $X$ be a $\mathbb{C}$-scheme. Then there exists a sheaf $\mathcal{S}_{X}$ of $\mathbb{C}$ vector spaces on $X$, unique up to canonical isomorphism, which is uniquely characterized by the following two properties:

(i) Suppose $R \subseteq X$ is Zariski open, $U$ is a smooth $\mathbb{C}$-scheme, and $i: R \hookrightarrow U$ is a closed embedding. Then we have an exact sequence of sheaves of $\mathbb{C}$-vector spaces on $R$ :

$$
\left.0 \longrightarrow I_{R, U} \longrightarrow i^{-1}\left(\mathcal{O}_{U}\right) \stackrel{i^{\sharp}}{\longrightarrow} \mathcal{O}_{X}\right|_{R} \longrightarrow 0,
$$

where $\mathcal{O}_{X}, \mathcal{O}_{U}$ are the sheaves of regular functions on $X, U$, and $i^{\sharp}$ is the morphism of sheaves of $\mathbb{C}$-algebras on $R$ induced by $i$.

There is an exact sequence of sheaves of $\mathbb{C}$-vector spaces on $R$ :

$$
\left.0 \longrightarrow \mathcal{S}_{X}\right|_{R} \stackrel{\iota_{R, U}}{\longrightarrow} \frac{i^{-1}\left(\mathcal{O}_{U}\right)}{I_{R, U}^{2}} \longrightarrow \mathrm{d}_{\longrightarrow} \frac{i^{-1}\left(T^{*} U\right)}{I_{R, U} \cdot i^{-1}\left(T^{*} U\right)}
$$

where d maps $f+I_{R, U}^{2} \mapsto \mathrm{d} f+I_{R, U} \cdot i^{-1}\left(T^{*} U\right)$. 
(ii) Let $R \subseteq S \subseteq X$ be Zariski open, $U, V$ be smooth $\mathbb{C}$-schemes, $i: R \hookrightarrow U$, $j: S \hookrightarrow V$ closed embeddings, and $\Phi: U \rightarrow V$ a morphism with $\Phi \circ i=$ $\left.j\right|_{R}: R \rightarrow V$. Then the following diagram of sheaves on $R$ commutes:

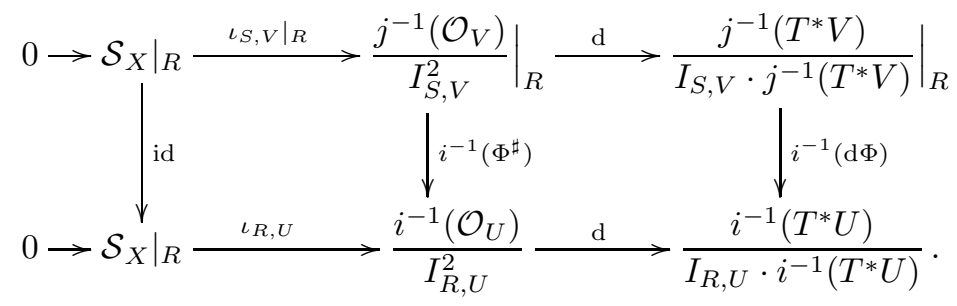

Here $\Phi: U \rightarrow V$ induces $\Phi^{\sharp}: \Phi^{-1}\left(\mathcal{O}_{V}\right) \rightarrow \mathcal{O}_{U}$ on $U$, so we have

$$
i^{-1}\left(\Phi^{\sharp}\right):\left.j^{-1}\left(\mathcal{O}_{V}\right)\right|_{R}=i^{-1} \circ \Phi^{-1}\left(\mathcal{O}_{V}\right) \longrightarrow i^{-1}\left(\mathcal{O}_{U}\right),
$$

a morphism of sheaves of $\mathbb{C}$-algebras on $R$. As $\Phi \circ i=\left.j\right|_{R}$, equation (6.2) maps $\left.I_{S, V}\right|_{R} \rightarrow I_{R, U}$, and so maps $\left.I_{S, V}^{2}\right|_{R} \rightarrow I_{R, U}^{2}$. Thus (6.2) induces the morphism in the second column of (6.1). Similarly, $\mathrm{d} \Phi: \Phi^{-1}\left(T^{*} V\right) \rightarrow$ $T^{*} U$ induces the third column of (6.1).

There is a natural decomposition $\mathcal{S}_{X}=\mathcal{S}_{X}^{0} \oplus \mathbb{C}_{X}$, where $\mathbb{C}_{X}$ is the constant sheaf on $X$ with fibre $\mathbb{C}$, and $\mathcal{S}_{X}^{0} \subset \mathcal{S}_{X}$ is the kernel of the composition

$$
\mathcal{S}_{X} \stackrel{\beta_{X}}{\longrightarrow} \mathcal{O}_{X} \stackrel{i_{X}^{\sharp}}{\longrightarrow} \mathcal{O}_{X^{\mathrm{red}}},
$$

with $X^{\mathrm{red}}$ the reduced $\mathbb{C}$-subscheme of $X$, and $i_{X}: X^{\mathrm{red}} \hookrightarrow X$ the inclusion.

The analogue of all the above also holds for complex analytic spaces.

Definition 6.2. An algebraic $d$-critical locus over $\mathbb{C}$ is a pair $(X, s)$, where $X$ is a $\mathbb{C}$-scheme, and $s \in H^{0}\left(\mathcal{S}_{X}^{0}\right)$ for $\mathcal{S}_{X}^{0}$ as in Theorem 6.1, satisfying the condition that for each $x \in X$, there exists a Zariski open neighbourhood $R$ of $x$ in $X$, a smooth $\mathbb{C}$-scheme $U$, a regular function $f: U \rightarrow \mathbb{A}^{1}=\mathbb{C}$, and a closed embedding $i: R \hookrightarrow U$, such that $i(R)=\operatorname{Crit}(f)$ as $\mathbb{C}$-subschemes of $U$, and $\iota_{R, U}\left(\left.s\right|_{R}\right)=i^{-1}(f)+I_{R, U}^{2}$.

Similarly, a complex analytic d-critical locus is a pair $(X, s)$, where $X$ is a complex analytic space, and $s \in H^{0}\left(\mathcal{S}_{X}^{0}\right)$ for $\mathcal{S}_{X}$ as in Theorem 6.1 such that each $x \in X$ has an open neighbourhood $R \subset X$ with a closed embedding $i: R \hookrightarrow U$ into a complex manifold $U$ and a holomorphic function $f: U \rightarrow \mathbb{C}$, such that $i(R)=\operatorname{Crit}(f)$, and $\iota_{R, U}\left(\left.s\right|_{R}\right)=i^{-1}(f)+I_{R, U}^{2}$.

In both cases we call the quadruple $(R, U, f, i)$ a critical chart on $(X, s)$.

Let $(X, s)$ be a d-critical locus (either algebraic or complex analytic), and $(R, U, f, i)$ be a critical chart on $(X, s)$. Let $U^{\prime} \subseteq U$ be (Zariski) open, and set $R^{\prime}=i^{-1}\left(U^{\prime}\right) \subseteq R, i^{\prime}=\left.i\right|_{R^{\prime}}: R^{\prime} \hookrightarrow U^{\prime}$, and $f^{\prime}=\left.f\right|_{U^{\prime}}$. Then $\left(R^{\prime}, U^{\prime}, f^{\prime}, i^{\prime}\right)$ is also a critical chart on $(X, s)$, and we call it a subchart of $(R, U, f, i)$. As a shorthand we write $\left(R^{\prime}, U^{\prime}, f^{\prime}, i^{\prime}\right) \subseteq(R, U, f, i)$. 
Let $(R, U, f, i),(S, V, g, j)$ be critical charts on $(X, s)$, with $R \subseteq S \subseteq X$. An embedding of $(R, U, f, i)$ in $(S, V, g, j)$ is a locally closed embedding $\Phi: U \hookrightarrow V$ such that $\Phi \circ i=\left.j\right|_{R}$ and $f=g \circ \Phi$. As a shorthand we write $\Phi:(R, U, f, i) \hookrightarrow$ $(S, V, g, j)$. If $\Phi:(R, U, f, i) \hookrightarrow(S, V, g, j)$ and $\Psi:(S, V, g, j) \hookrightarrow(T, W, h, k)$ are embeddings, then $\Psi \circ \Phi:(R, U, f, i) \hookrightarrow(T, W, h, k)$ is also an embedding.

Theorem 6.3. Let $(X, s)$ be a d-critical locus (either algebraic or complex analytic), and $(R, U, f, i),(S, V, g, j)$ be critical charts on $(X, s)$. Then for each $x \in R \cap S \subseteq X$ there exist subcharts $\left(R^{\prime}, U^{\prime}, f^{\prime}, i^{\prime}\right) \subseteq(R, U, f, i),\left(S^{\prime}, V^{\prime}, g^{\prime}, j^{\prime}\right) \subseteq$ $(S, V, g, j)$ with $x \in R^{\prime} \cap S^{\prime} \subseteq X$, a critical chart $(T, W, h, k)$ on $(X, s)$, and embeddings $\Phi:\left(R^{\prime}, U^{\prime}, f^{\prime}, i^{\prime}\right) \hookrightarrow(T, W, h, k), \Psi:\left(S^{\prime}, V^{\prime}, g^{\prime}, j^{\prime}\right) \hookrightarrow(T, W, h, k)$.

Theorem 6.4. Let $(X, s)$ be a d-critical locus (either algebraic or complex analytic), and $X^{\text {red }} \subseteq X$ the associated reduced $\mathbb{C}$-scheme or reduced complex analytic space. Then there exists an (algebraic or holomorphic) line bundle $K_{X, s}$ on $X^{\mathrm{red}}$ which we call the canonical bundle of $(X, s)$, which is natural up to canonical isomorphism, and is characterized by the following properties:

(i) If $(R, U, f, i)$ is a critical chart on $(X, s)$, there is a natural isomorphism

$$
\iota_{R, U, f, i}:\left.\left.K_{X, s}\right|_{R^{\mathrm{red}}} \longrightarrow i^{*}\left(K_{U}^{\otimes^{2}}\right)\right|_{R^{\mathrm{red}}},
$$

where $K_{U}=\Lambda^{\operatorname{dim} U} T^{*} U$ is the canonical bundle of $U$ in the usual sense.

(ii) Let $\Phi:(R, U, f, i) \hookrightarrow(S, V, g, j)$ be an embedding of critical charts on $(X, s)$. Then (5.9) defines an isomorphism of line bundles on $\operatorname{Crit}(f)^{\mathrm{red}}$ :

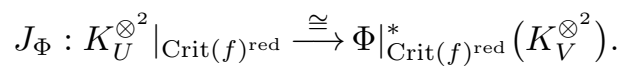

Since $i: R \rightarrow \operatorname{Crit}(f)$ is an isomorphism with $\Phi \circ i=\left.j\right|_{R}$, this gives

$$
\left.i\right|_{R^{\text {red }}} ^{*}\left(J_{\Phi}\right):\left.\left.i\right|_{R^{\text {red }}} ^{*}\left(K_{U}^{\otimes^{2}}\right) \stackrel{\cong}{\longrightarrow} j\right|_{R^{\text {red }}} ^{*}\left(K_{V}^{\otimes^{2}}\right),
$$

and we must have

$$
\iota_{S, V, g, j}||_{R^{\mathrm{red}}}=\left.i\right|_{R^{\mathrm{red}}} ^{*}\left(J_{\Phi}\right) \circ \iota_{R, U, f, i}:\left.\left.K_{X, s}\right|_{R^{\mathrm{red}}} \longrightarrow j^{*}\left(K_{V}^{\otimes^{2}}\right)\right|_{R^{\mathrm{red}}} .
$$

Definition 6.5. Let $(X, s)$ be a d-critical locus (either algebraic or complex analytic), and $K_{X, s}$ its canonical bundle from Theorem 6.4. An orientation on $(X, s)$ is a choice of square root line bundle $K_{X, s}^{1 / 2}$ for $K_{X, s}$ on $X^{\text {red }}$. That is, an orientation is an (algebraic or holomorphic) line bundle $L$ on $X^{\text {red }}$, together with an isomorphism $L^{\otimes^{2}}=L \otimes L \cong K_{X, s}$. A d-critical locus with an orientation will be called an oriented d-critical locus.

In [9, Th. 6.6] we show that algebraic d-critical loci are classical truncations of objects in derived algebraic geometry known as -1-shifted symplectic derived schemes, introduced by Pantev, Toën, Vaquié and Vezzosi 42. 
Theorem 6.6 (Bussi, Brav and Joyce [9]). Suppose $(\boldsymbol{X}, \omega)$ is a-1-shifted symplectic derived scheme in the sense of Pantev et al. [42] over $\mathbb{C}$, and let $X=$ $t_{0}(\boldsymbol{X})$ be the associated classical $\mathbb{C}$-scheme of $\boldsymbol{X}$. Then $X$ extends naturally to an algebraic d-critical locus $(X, s)$. The canonical bundle $K_{X, s}$ from Theorem 6.4 is naturally isomorphic to the determinant line bundle $\left.\operatorname{det}\left(\mathbb{L}_{\boldsymbol{X}}\right)\right|_{X^{\text {red }}}$ of the cotangent complex $\mathbb{L}_{\boldsymbol{X}}$ of $\boldsymbol{X}$.

Now Pantev et al. [4] show that derived moduli schemes of coherent sheaves, or complexes of coherent sheaves, on a Calabi-Yau 3-fold $Y$ have -1-shifted symplectic structures. Using this, in [9, Cor. 6.7] we deduce:

Corollary 6.7. Suppose $Y$ is a Calabi-Yau 3 -fold over $\mathbb{C}$, and $\mathcal{M}$ is a classical moduli $\mathbb{C}$-scheme of simple coherent sheaves in $\operatorname{coh}(Y)$, or simple complexes of coherent sheaves in $D^{b} \operatorname{coh}(Y)$, with (symmetric) obstruction theory $\phi: \mathcal{E}^{\bullet} \rightarrow$ $\mathbb{L}_{\mathcal{M}}$ as in Behrend 2], Thomas [52, or Huybrechts and Thomas [22]. Then $\mathcal{M}$ extends naturally to an algebraic d-critical locus $(\mathcal{M}, s)$. The canonical bundle $K_{\mathcal{M}, s}$ from Theorem 6.4 is naturally isomorphic to $\left.\operatorname{det}\left(\mathcal{E}^{\bullet}\right)\right|_{\mathcal{M}^{\mathrm{red}}}$.

Here we call $F \in \operatorname{coh}(Y)$ simple if $\operatorname{Hom}(F, F)=\mathbb{C}$, and $F^{\bullet} \in D^{b} \operatorname{coh}(Y)$ simple if $\operatorname{Hom}\left(F^{\bullet}, F^{\bullet}\right)=\mathbb{C}$ and $\operatorname{Ext}^{<0}\left(F^{\bullet}, F^{\bullet}\right)=0$. Thus, d-critical loci will have applications in Donaldson-Thomas theory for Calabi-Yau 3-folds [24, 32, 33, 52. Orientations on $(\mathcal{M}, s)$ are closely related to orientation data in the work of Kontsevich and Soibelman 32,33 .

Pantev et al. 42. also show that derived intersections $L \cap M$ of algebraic Lagrangians $L, M$ in an algebraic symplectic manifold $(S, \omega)$ have -1 -shifted symplectic structures, so that Theorem 6.6 gives them the structure of algebraic d-critical loci. Bussi [10, §3] will prove a complex analytic version of this:

Theorem 6.8 (Bussi [10]). Suppose $(S, \omega)$ is a complex symplectic manifold, and $L, M$ are complex Lagrangian submanifolds in $S$. Then the intersection $X=L \cap M$, as a complex analytic subspace of $S$, extends naturally to a complex analytic d-critical locus $(X, s)$. The canonical bundle $K_{X, s}$ from Theorem 6.4 is naturally isomorphic to $\left.\left.K_{L}\right|_{X^{\text {red }}} \otimes K_{M}\right|_{X^{\text {red }}}$.

\subsection{The main result, and applications}

Here is our main result, which will be proved in $\sqrt[6.3]{6.4}$,

Theorem 6.9. Let $(X, s)$ be an oriented algebraic d-critical locus over $\mathbb{C}$, with orientation $K_{X, s}^{1 / 2}$. Then for any well-behaved base ring $A$, such as $\mathbb{Z}, \mathbb{Q}$ or $\mathbb{C}$, there exists a perverse sheaf $P_{X, s}^{\bullet}$ in $\operatorname{Perv}(X)$ over $A$, which is natural up to canonical isomorphism, and Verdier duality and monodromy isomorphisms

$$
\Sigma_{X, s}: P_{X, s}^{\bullet} \longrightarrow \mathbb{D}_{X}\left(P_{X, s}^{\bullet}\right), \quad \mathrm{T}_{X, s}: P_{X, s}^{\bullet} \longrightarrow P_{X, s}^{\bullet},
$$

which are characterized by the following properties:

(i) If $(R, U, f, i)$ is a critical chart on $(X, s)$, there is a natural isomorphism

$$
\omega_{R, U, f, i}:\left.P_{X, s}^{\bullet}\right|_{R} \longrightarrow i^{*}\left(\mathcal{P} \mathcal{V}_{U, f}^{\bullet}\right) \otimes_{\mathbb{Z} / 2 \mathbb{Z}} Q_{R, U, f, i},
$$


where $\pi_{R, U, f, i}: Q_{R, U, f, i} \rightarrow R$ is the principal $\mathbb{Z} / 2 \mathbb{Z}$-bundle parametrizing local isomorphisms $\alpha:\left.K_{X, s}^{1 / 2} \rightarrow i^{*}\left(K_{U}\right)\right|_{R^{\text {red }}}$ with $\alpha \otimes \alpha=\iota_{R, U, f, i}$, for $\iota_{R, U, f, i}$ as in (6.3). Furthermore the following commute in $\operatorname{Perv}(R)$ :

$$
\begin{aligned}
& \left.P_{X, s}^{\bullet}\right|_{R} i^{*}\left(\mathcal{P} \mathcal{V}_{U, f}^{\bullet}\right) \otimes_{\mathbb{Z} / 2 \mathbb{Z}} Q_{R, U, f, i} \\
& \left.\sum_{X, s}\right|_{R} \quad i^{*}\left(\sigma_{U, f}\right) \otimes \operatorname{id}_{Q_{R, U, f, i}} \downarrow \\
& \begin{aligned}
\mathbb{D}_{R}\left(\left.P_{X, s}^{\bullet}\right|_{R}\right) \longleftarrow \mathbb{D}_{R}\left(\omega_{R, U, f, i}\right) & i^{*} \\
& \left(\mathbb{D}_{\operatorname{Crit}(f)}\left(\mathcal{P} \mathcal{V}_{U, f}^{\bullet}\right)\right) \otimes_{\mathbb{Z} / 2 \mathbb{Z}} Q_{R, U, f, i} \\
& \cong \mathbb{D}_{R}\left(i^{*}\left(\mathcal{P} \mathcal{V}_{U, f}^{\bullet}\right) \otimes_{\mathbb{Z} / 2 \mathbb{Z}} Q_{R, U, f, i}\right),
\end{aligned}
\end{aligned}
$$

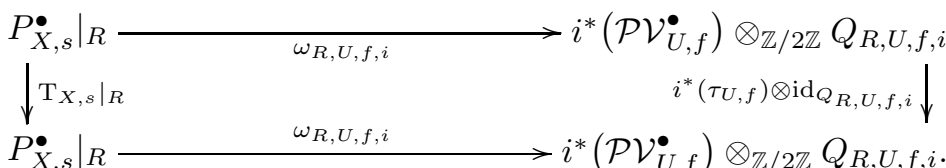

(ii) Let $\Phi:(R, U, f, i) \hookrightarrow(S, V, g, j)$ be an embedding of critical charts on $(X, s)$. Then there is a natural isomorphism of principal $\mathbb{Z} / 2 \mathbb{Z}$-bundles

$$
\Lambda_{\Phi}:\left.Q_{S, V, g, j}\right|_{R} \stackrel{\cong}{\cong} i^{*}\left(P_{\Phi}\right) \otimes_{\mathbb{Z} / 2 \mathbb{Z}} Q_{R, U, f, i}
$$

on $R$, for $P_{\Phi}$ as in Definition 5.2, defined as follows: local isomorphisms

$$
\begin{gathered}
\alpha:\left.\left.K_{X, s}^{1 / 2}\right|_{R^{\mathrm{red}}} \longrightarrow i^{*}\left(K_{U}\right)\right|_{R^{\mathrm{red}}, \quad \beta:\left.\left.K_{X, s}^{1 / 2}\right|_{R^{\mathrm{red}}} \longrightarrow j^{*}\left(K_{V}\right)\right|_{R^{\mathrm{red}}},} \quad, \quad \gamma:\left.\left.i^{*}\left(K_{U}\right)\right|_{R^{\mathrm{red}}} \longrightarrow j^{*}\left(K_{V}\right)\right|_{R^{\mathrm{red}}}
\end{gathered}
$$

with $\alpha \otimes \alpha=\iota_{R, U, f, i}, \beta \otimes \beta=\left.\iota_{S, V, g, j}\right|_{R^{\mathrm{red}}}, \gamma \otimes \gamma=\left.i\right|_{R^{\text {red }}} ^{*}\left(J_{\Phi}\right)$ correspond to local sections $s_{\alpha}: R \rightarrow Q_{R, U, f, i}, s_{\beta}:\left.R \rightarrow Q_{S, V, g, j}\right|_{R}, s_{\gamma}: R \rightarrow i^{*}\left(P_{\Phi}\right)$. Equation (6.4) shows that $\beta=\gamma \circ \alpha$ is a possible solution for $\beta$, and we define $\Lambda_{\Phi}$ in (6.9) such that $\Lambda_{\Phi}\left(s_{\beta}\right)=s_{\gamma} \otimes_{\mathbb{Z} / 2 \mathbb{Z}} s_{\alpha}$ if and only if $\beta=\gamma \circ \alpha$. Then the following diagram commutes in $\operatorname{Perv}(R)$, for $\Theta_{\Phi}$ as in (5.13):

$$
\begin{aligned}
& \left.P_{X, s}^{\bullet}\right|_{R} \longrightarrow i^{*}\left(\mathcal{P} \mathcal{V}_{U, f}^{\bullet}\right) \otimes_{\mathbb{Z} / 2 \mathbb{Z}} Q_{R, U, f, i} \\
& \left|\omega_{S, V, g, j}\right|_{R} \quad i^{*}\left(\Theta_{\Phi}\right) \otimes \operatorname{id}_{Q_{R, U, f, i}} \downarrow \\
& \left.j^{*}\left(\mathcal{P} \mathcal{V}_{V, g}^{\bullet}\right)\right|_{R} \quad \stackrel{\operatorname{id}_{j^{*}\left(\mathcal{P} V_{V, g}^{\bullet}\right.} \otimes \Lambda_{\Phi}}{\longrightarrow} i^{*}\left(\Phi^{*}\left(\mathcal{P} \mathcal{V}_{V, g}^{\bullet}\right) \otimes_{\mathbb{Z} / 2 \mathbb{Z}} P_{\Phi}\right) \\
& \left.\otimes_{\mathbb{Z} / 2 \mathbb{Z}} Q_{S, V, g, j}\right|_{R} \longrightarrow \otimes_{\mathbb{Z} / 2 \mathbb{Z}} Q_{R, U, f, i} \text {. }
\end{aligned}
$$

The analogues of all the above also hold for $\mathscr{D}$-modules on oriented algebraic $d$-critical loci over $\mathbb{C}$, for perverse sheaves and $\mathscr{D}$-modules on oriented complex analytic d-critical loci, and for mixed Hodge modules on oriented algebraic $d$ critical loci over $\mathbb{C}$ and oriented complex analytic d-critical loci, as in $\$ 2.6-\$ 2.10$.

Remark 6.10. This sheaf-theoretic result is compatible with the motivic result of Bussi, Joyce and Meinhardt in [11. Given $(X, s)$ an oriented algebraic d-critical locus over $\mathbb{C}$, 11 proves the existence of a natural motivic element 
$M F_{X, s} \in \overline{\mathcal{M}}_{X}^{\hat{\mu}}$ in a version of the relative Grothendieck ring of varieties over $X$, equivariant with respect to suitable actions of the group $\hat{\mu}$ of all roots of unity (for detailed definitions, see [11]). Since the mixed Hodge module realization factorizes over the additional relation one has to impose in [11] on the Grothendieck group, the ring $\mathcal{M}_{X}^{\hat{\mu}}$ has a map to $K_{0}\left(\operatorname{MHM}\left(X ; T_{s}\right)\right)$, the $K$-group of algebraic mixed Hodge modules on $X$ with a finite order automorphism (note that the Grothendieck group only sees the semisimple part $T_{s}$ of the monodromy and not the nilpotent part $N$ ). By a Cech-type argument using the corresponding comparison result of [20, Prop. 3.17], the image of $M F_{X, s}$ in $K_{0}\left(\operatorname{MHM}\left(X ; T_{s}\right)\right)$ agrees with the image of the mixed Hodge module realization of $P_{X, s}^{\bullet}$, since both sides are Zariski locally modelled by the same vanishing cycles. Thus, for example, they give the same weight polynomial for global cohomology with compact support.

From Theorem 6.6. Corollary 6.7 and Theorem 6.8 we deduce:

Corollary 6.11. Let $(\boldsymbol{X}, \omega)$ be a-1-shifted symplectic derived scheme over $\mathbb{C}$ in the sense of Pantev et al. [42, and $X=t_{0}(\boldsymbol{X})$ the associated classical $\mathbb{C}$ scheme. Suppose we are given a square root $\left.\operatorname{det}\left(\mathbb{L}_{\boldsymbol{X}}\right)\right|_{X} ^{1 / 2}$ for $\left.\operatorname{det}\left(\mathbb{L}_{\boldsymbol{X}}\right)\right|_{X}$. Then we may define $P_{\boldsymbol{X}, \omega}^{\bullet} \in \operatorname{Perv}(X)$, uniquely up to canonical isomorphism, and isomorphisms $\Sigma_{\boldsymbol{X}, \omega}: P_{\boldsymbol{X}, \omega}^{\bullet} \rightarrow \mathbb{D}_{X}\left(P_{\boldsymbol{X}, \omega}^{\bullet}\right), \mathrm{T}_{\boldsymbol{X}, \omega}: P_{\boldsymbol{X}, \omega}^{\bullet} \rightarrow P_{\boldsymbol{X}, \omega}^{\bullet}$.

The same applies for $\mathscr{D}$-modules and mixed Hodge modules on $X$.

Corollary 6.12. Let $Y$ be a Calabi-Yau 3-fold over $\mathbb{C}$, and $\mathcal{M}$ a classical moduli $\mathbb{C}$-scheme of simple coherent sheaves in $\operatorname{coh}(Y)$, or simple complexes of coherent sheaves in $D^{b} \operatorname{coh}(Y)$, with natural (symmetric) obstruction theory $\phi: \mathcal{E}^{\bullet} \rightarrow \mathbb{L}_{\mathcal{M}}$ as in Behrend [2, Thomas [52, or Huybrechts and Thomas [22. Suppose we are given a square root $\operatorname{det}\left(\mathcal{E}^{\bullet}\right)^{1 / 2}$ for $\operatorname{det}\left(\mathcal{E}^{\bullet}\right)$. Then we may define $P_{\mathcal{M}} \in \operatorname{Perv}(\mathcal{M})$, uniquely up to canonical isomorphism, and isomorphisms $\Sigma_{\mathcal{M}}$ : $P_{\mathcal{M}}^{\bullet} \rightarrow \mathbb{D}_{\mathcal{M}}\left(P_{\mathcal{M}}^{\bullet}\right), \mathrm{T}_{\mathcal{M}}: P_{\mathcal{M}}^{\bullet} \rightarrow P_{\mathcal{M}}$

The same applies for $\mathscr{D}$-modules and mixed Hodge modules on $\mathcal{M}$.

Corollary 6.13. Let $(S, \omega)$ be a complex symplectic manifold and $L, M$ complex Lagrangian submanifolds in $S$, and write $X=L \cap M$, as a complex analytic subspace of $S$. Suppose we are given square roots $K_{L}^{1 / 2}, K_{M}^{1 / 2}$ for $K_{L}, K_{M}$. Then we may define $P_{L, M}^{\bullet} \in \operatorname{Perv}(X)$, uniquely up to canonical isomorphism, and isomorphisms $\Sigma_{L, M}: P_{L, M}^{\bullet} \rightarrow \mathbb{D}_{X}\left(P_{L, M}^{\bullet}\right), \mathrm{T}_{L, M}: P_{L, M}^{\bullet} \rightarrow P_{L, M}^{\bullet}$.

The same applies for $\mathscr{D}$-modules and mixed Hodge modules on $X$.

The next two remarks discuss applications of Corollaries 6.12 and 6.13 to Donaldson-Thomas theory, and to Lagrangian Floer cohomology.

Remark 6.14. If $Y$ is a Calabi-Yau 3-fold over $\mathbb{C}$ and $\tau$ a suitable stability condition on coherent sheaves on $Y$, the Donaldson-Thomas invariants $D T^{\alpha}(\tau)$ are integers which 'count' the moduli schemes $\mathcal{M}_{\mathrm{st}}^{\alpha}(\tau)$ of $\tau$-stable coherent sheaves on $Y$ with Chern character $\alpha \in H^{\text {even }}(Y ; \mathbb{Q})$, provided there are no strictly $\tau$-semistable sheaves in class $\alpha$ on $Y$. They were defined by Thomas [52, who 
showed they are unchanged under deformations of $Y$, following a suggestion of Donaldson and Thomas 16 .

Behrend [2] showed that $D T^{\alpha}(\tau)$ may be written as a weighted Euler characteristic $\chi\left(\mathcal{M}_{\mathrm{st}}^{\alpha}(\tau), \nu\right)$, where $\nu: \mathcal{M}_{\mathrm{st}}^{\alpha}(\tau) \rightarrow \mathbb{Z}$ is a certain constructible function called the Behrend function. Joyce and Song 24] extended the definition of $D T^{\alpha}(\tau)$ to classes $\alpha$ including $\tau$-semistable sheaves (with $D T^{\alpha}(\tau) \in \mathbb{Q}$ ), and proved a wall-crossing formula for $D T^{\alpha}(\tau)$ under change of stability condition $\tau$. Kontsevich and Soibelman 32 gave a (partly conjectural) motivic generalization of Donaldson-Thomas invariants, also with a wall-crossing formula.

Corollary 6.12 is relevant to the categorification of Donaldson-Thomas theory. As in [2, §1.2], the perverse sheaf $P_{\mathcal{M}_{\mathrm{st}}^{\alpha}(\tau)}^{\bullet}$ has pointwise Euler characteristic $\chi\left(P_{\mathcal{M}_{\mathrm{st}}^{\alpha}(\tau)}\right)=\nu$. This implies that when $A$ is a field, say $A=\mathbb{Q}$, the (compactlysupported) hypercohomologies $\mathbb{H}^{*}\left(P_{\mathcal{M}_{\mathrm{st}}^{\alpha}(\tau)}^{\boldsymbol{N}^{\alpha}}\right), \mathbb{H}_{\mathrm{c}}^{*}\left(P_{\mathcal{M}_{\mathrm{st}}^{\alpha}(\tau)}^{\bullet^{\alpha}}\right)$ from (2.1) satisfy

$$
\begin{aligned}
\sum_{k \in \mathbb{Z}}(-1)^{k} \operatorname{dim} \mathbb{H}^{k}\left(P_{\mathcal{M}_{\mathrm{st}}^{\alpha}(\tau)}\right) & =\sum_{k \in \mathbb{Z}}(-1)^{k} \operatorname{dim} \mathbb{H}_{\mathrm{c}}^{k}\left(P_{\mathcal{M}_{\mathrm{st}}^{\alpha}(\tau)}\right) \\
& =\chi\left(\mathcal{M}_{\mathrm{st}}^{\alpha}(\tau), \nu\right)=D T^{\alpha}(\tau),
\end{aligned}
$$

where $\mathbb{H}^{k}\left(P_{\mathcal{M}_{\mathrm{st}}^{\alpha}(\tau)}^{\boldsymbol{c}^{\alpha}}\right) \cong \mathbb{H}_{\mathrm{c}}^{-k}\left(P_{\mathcal{M}_{\mathrm{st}}^{\alpha}(\tau)}^{\bullet^{*}}\right)^{*}$ by Verdier duality. That is, we have produced a natural graded $\mathbb{Q}$-vector space $\mathbb{H}^{*}\left(P_{\mathcal{M}_{\mathrm{st}}^{\alpha}(\tau)}\right)$, thought of as some kind of generalized cohomology of $\mathcal{M}_{\mathrm{st}}^{\alpha}(\tau)$, whose graded dimension is $D T^{\alpha}(\tau)$. This gives a new interpretation of the Donaldson-Thomas invariant $D T^{\alpha}(\tau)$.

In fact, as discussed at length in [51, §3], the first natural "refinement" or "quantization" direction of a Donaldson-Thomas invariant $D T^{\alpha}(\tau) \in \mathbb{Z}$ is not the Poincaré polynomial of this cohomology, but its weight polynomial

$$
w\left(\mathbb{H}^{*}\left(P_{\mathcal{M}_{\mathrm{st}}^{\alpha}(\tau)}\right), t\right) \in \mathbb{Z}\left[t^{ \pm \frac{1}{2}}\right],
$$

defined using the mixed Hodge structure on the cohomology of the mixed Hodge module version of $P_{\mathcal{M}_{\mathrm{st}}^{\alpha}(\tau)}$ (which exists assuming that $\mathcal{M}_{\mathrm{st}}^{\alpha}(\tau)$ is projective, for example, see Remark 2.22).

The material above is related to work by other authors. The idea of categorifying Donaldson-Thomas invariants using perverse sheaves or $\mathscr{D}$-modules is probably first due to Behrend [2, and for Hilbert schemes $\operatorname{Hilb}^{n}(Y)$ of a CalabiYau 3-fold $Y$ is discussed by Dimca and Szendrői [15] and Behrend, Bryan and Szendrői [3, §3.4], using mixed Hodge modules. Corollary 6.12 answers a question of Joyce and Song [24, Question 5.7(a)].

As in [24, 32] representations of quivers with superpotentials $(Q, W)$ give 3-Calabi-Yau triangulated categories, and one can define Donaldson-Thomas type invariants $D T_{Q, W}^{\alpha}(\tau)$ 'counting' such representations, which are simple algebraic 'toy models' for Donaldson-Thomas invariants of Calabi-Yau 3-folds. Kontsevich and Soibelman 33 explain how to categorify these quiver invariants $D T_{Q, W}^{\alpha}(\tau)$, and define an associative multiplication on the categorification to make a Cohomological Hall Algebra. This paper was motivated by the aim of extending [33] to define Cohomological Hall Algebras for Calabi-Yau 3-folds. 
The square root $\operatorname{det}\left(\mathcal{E}^{\bullet}\right)^{1 / 2}$ required in Corollary 6.12 corresponds roughly to orientation data in the work of Kontsevich and Soibelman [32, §5], 33.

In a paper written independently of our programme [9, 11, 23, Kiem and Li [31] have recently proved an analogue of Corollary 6.12 by complex analytic methods, beginning from Joyce and Song's result [24, Th. 5.4], proved using gauge theory, that $\mathcal{M}_{\mathrm{st}}^{\alpha}(\tau)$ is locally isomorphic to $\operatorname{Crit}(f)$ as a complex analytic space, for $V$ a complex manifold and $f: V \rightarrow \mathbb{C}$ holomorphic.

Remark 6.15. In the situation of Corollary 6.13, with $\operatorname{dim}_{\mathbb{C}} S=2 n$, we claim that there ought morally to be some kind of approximate comparison

$$
\mathbb{H}^{k}\left(P_{L, M}^{\bullet}\right) \approx H F^{k+n}(L, M),
$$

where $H F^{*}(L, M)$ is the Lagrangian Floer cohomology of Fukaya, Oh, Ohta and Ono [18. We can compare and contrast the two sides of (6.11) as follows:

(a) $\mathbb{H}^{*}\left(P_{L, M}^{\bullet}\right)$ is defined over any well-behaved base ring $A$, e.g. $A=\mathbb{Z}$ or $\mathbb{Q}$, but $H F^{*}(L, M)$ is defined over a Novikov ring of power series $\Lambda_{\text {nov }}$.

(b) $\mathbb{H}^{*}\left(P_{L, M}^{\bullet}\right)$ has extra structure not visible in $H F^{*}(L, M)$, from Verdier duality and monodromy operators $\Sigma_{L, M}, \mathrm{~T}_{L, M}$, plus the mixed Hodge module version has a mixed Hodge structure.

(c) $\mathbb{H}^{*}\left(P_{L, M}^{\bullet}\right)$ is defined for arbitrary complex Lagrangians $L, M$, not necessarily compact or closed in $S$, but $H F^{*}(L, M)$ is only defined for $L, M$ compact, or at least for $L, M$ closed and well-behaved at infinity.

(d) To define $H F^{*}(L, M)$ one generally assumes $L, M$ intersect transversely, or at least cleanly. But $\mathbb{H}^{*}\left(P_{L, M}^{\bullet}\right)$ is defined when $L \cap M$ is arbitrarily singular, and the construction is only really interesting for singular $L \cap M$.

(e) To define $H F^{*}(L, M)$ we need $L, M$ to be oriented and spin, to orient moduli spaces of $J$-holomorphic curves. When $L, M$ are complex Lagrangians they are automatically oriented, and spin structures on $L, M$ correspond to choices of square roots $K_{L}^{1 / 2}, K_{M}^{1 / 2}$, as used in Corollary 6.13,

Some of the authors are working on defining a 'Fukaya category' of complex Lagrangians in a complex symplectic manifold, using $\mathbb{H}^{*}\left(P_{L, M}^{\bullet}\right)$ as morphisms.

We now discuss related work. Nadler and Zaslow [40, 41, show that if $X$ is a real analytic manifold (for instance, a complex manifold), then the derived category $D_{c}^{b}(X)$ of constructible sheaves on $X$ is equivalent to a certain derived Fukaya category $D^{b} \mathcal{F}\left(T^{*} X\right)$ of exact Lagrangians in $T^{*} X$.

Let $L, M$ be complex Lagrangians in a complex symplectic manifold $(S, \omega)$. Regarding $\mathcal{O}_{L}, \mathcal{O}_{M}$ as coherent sheaves on $S$, Behrend and Fantechi 4 , Th.s 4.3 $\& 5.2]$ claim to construct canonical $\mathbb{C}$-linear (not $\mathcal{O}_{S}$-linear) differentials

$$
\mathrm{d}: \mathcal{E}_{x} t_{\mathcal{O}_{S}}^{i}\left(\mathcal{O}_{L}, \mathcal{O}_{M}\right) \longrightarrow \mathcal{E} x t_{\mathcal{O}_{S}}^{i+1}\left(\mathcal{O}_{L}, \mathcal{O}_{M}\right)
$$

with $\mathrm{d}^{2}=0$, such that $\left(\mathcal{E} x t_{\mathcal{O}_{S}}^{*}\left(\mathcal{O}_{L}, \mathcal{O}_{M}\right)\right.$, d $)$ is a constructible complex. There is a mistake in the proof of [4, Th. 4.3]. To fix this one should instead work with 
$\mathcal{E} x t_{\mathcal{O}_{S}}^{*}\left(K_{L}^{1 / 2}, K_{M}^{1 / 2}\right)$ for square roots $K_{L}^{1 / 2}, K_{M}^{1 / 2}$ as in Corollary 6.13. Also the proof of the constructibility of $\left(\mathcal{E} x t_{\mathcal{O}_{S}}^{*}\left(K_{L}^{1 / 2}, K_{M}^{1 / 2}\right)\right.$,d) in [4, Th. 5.2] depended on a result of Kapranov, which later turned out to be false.

Our $P_{L, M}^{\bullet}$ over $A=\mathbb{C}$ should be the natural perverse sheaf on $L \cap M$ conjectured by Behrend and Fantechi [4, Conj. 5.16], who also suggest there should be a spectral sequence from $\left(\mathcal{E} x t_{\mathcal{O}_{S}}^{*}\left(K_{L}^{1 / 2}, K_{M}^{1 / 2}\right)\right.$, d) $[n]$ to $P_{L, M}^{\bullet}$. (See Sabbah [44, Th. 1.1] for a related result.) In [4, §5.3], Behrend and Fantechi discuss how to define a 'Fukaya category' using their ideas.

Kashiwara and Schapira [29] develop a theory of deformation quantization modules, or $D Q$-modules, on a complex symplectic manifold $(S, \omega)$, which roughly may be regarded as symplectic versions of $\mathscr{D}$-modules. Holonomic DQmodules $\mathcal{D}^{\bullet}$ are supported on (possibly singular) complex Lagrangians $L$ in $S$. If $L$ is a smooth, closed, complex Lagrangian in $S$ and $K_{L}^{1 / 2}$ a square root of $K_{L}$, D'Agnolo and Schapira [13] show that there exists a simple holonomic DQ-module $\mathcal{D}^{\bullet}$ supported on $L$.

If $\mathcal{D}^{\bullet}, \mathcal{E}^{\bullet}$ are simple holonomic DQ-modules on $S$ supported on smooth Lagrangians $L, M$, then Kashiwara and Schapira [28] show that $R \mathscr{H} \circ \mathrm{om}\left(\mathcal{D}^{\bullet}, \mathcal{E}^{\bullet}\right)[n]$ is a perverse sheaf on $S$ over the field $\mathbb{C}((\hbar))$, supported on $X=L \cap M$. Pierre Schapira explained to the authors how to prove that $R \mathscr{H} \operatorname{om}\left(\mathcal{D}^{\bullet}, \mathcal{E}^{\bullet}\right)[n] \cong P_{L, M}^{\bullet}$, when $P_{L, M}^{\bullet}$ is defined over the base ring $A=\mathbb{C}((\hbar))$.

Now let $L, M, N$ be Lagrangians in $S$, with square roots $K_{L}^{1 / 2}, K_{M}^{1 / 2}, K_{N}^{1 / 2}$. We have a product $H F^{k}(L, M) \times H F^{l}(M, N) \rightarrow H F^{k+l}(L, N)$ from composition of morphisms in $D^{b} \mathcal{F}(S)$. So (6.11) suggests there should be a product

$$
\mathbb{H}^{k}\left(P_{L, M}^{\bullet}\right) \times \mathbb{H}^{l}\left(P_{M, N}^{\bullet}\right) \longrightarrow \mathbb{H}^{k+l+n}\left(P_{L, N}^{\bullet}\right),
$$

which would naturally be induced by a morphism in $D_{c}^{b}(S)$

$$
\mu_{L, M, N}: P_{L, M}^{\bullet} \stackrel{L}{\otimes} P_{M, N}^{\bullet} \longrightarrow P_{L, N}^{\bullet}[n] .
$$

Observe that the work of Behrend-Fantechi and Kashiwara-Schapira cited above supports the existence of (6.12)-(6.13): there are natural products

$$
\begin{aligned}
\mathcal{E} x t_{\mathcal{O}_{S}}^{k}\left(K_{L}^{1 / 2}, K_{M}^{1 / 2}\right) \otimes_{\mathcal{O}_{S}} \mathcal{E} x t_{\mathcal{O}_{S}}^{l}\left(K_{M}^{1 / 2}, K_{N}^{1 / 2}\right) & \longrightarrow \mathcal{E} x t_{\mathcal{O}_{S}}^{k+l}\left(K_{L}^{1 / 2}, K_{N}^{1 / 2}\right), \\
R \mathscr{H} \operatorname{om}\left(\mathcal{D}^{\bullet}, \mathcal{E}^{\bullet}\right) \stackrel{\otimes}{\otimes} R \mathscr{H} \operatorname{om}\left(\mathcal{E}^{\bullet}, \mathcal{F}^{\bullet}\right) & \longrightarrow R \mathscr{H} \text { om }\left(\mathcal{D}^{\bullet}, \mathcal{F}^{\bullet}\right) .
\end{aligned}
$$

But since (6.13) is a morphism of complexes, not of perverse sheaves, Theorem 2.7(i) does not apply, so we cannot construct $\mu_{L, M, N}$ by naïvely gluing data on an open cover, as we have been doing in $\sqrt{3}-\sqrt{6}$.

\subsection{Proof of Theorem 6.9 for $\mathbb{C}$-schemes}

Let $(X, s)$ be an oriented algebraic d-critical locus over $\mathbb{C}$, with orientation $K_{X, s}^{1 / 2}$. By Definition 6.2 we may choose a family $\left\{\left(R_{a}, U_{a}, f_{a}, i_{a}\right): a \in A\right\}$ of critical 
charts $\left(R_{a}, U_{a}, f_{a}, i_{a}\right)$ on $(X, s)$ such that $\left\{R_{a}: a \in A\right\}$ is a Zariski open cover of the $\mathbb{C}$-scheme $X$. Then for each $a \in A$ we have a perverse sheaf

$$
i_{a}^{*}\left(\mathcal{P} \mathcal{V}_{U_{a}, f_{a}}^{\bullet}\right) \otimes_{\mathbb{Z} / 2 \mathbb{Z}} Q_{R_{a}, U_{a}, f_{a}, i_{a}} \in \operatorname{Perv}\left(R_{a}\right),
$$

for $Q_{R_{a}, U_{a}, f_{a}, i_{a}}$ as in Theorem 6.9)(i). The idea of the proof is to use Theorem 2.7(ii) to glue the perverse sheaves (6.14) on the Zariski open cover $\left\{R_{a}: a \in A\right\}$ to get a global perverse sheaf $P_{X, s}^{\bullet}$ on $X$. Note that Theorem 2.7(ii) is written for étale open covers, but this immediately implies the simpler Zariski version.

To do this, for all $a, b \in A$ we have to construct isomorphisms

$$
\begin{aligned}
\alpha_{a b}: & {\left.\left[i_{a}^{*}\left(\mathcal{P} \mathcal{V}_{U_{a}, f_{a}}^{\bullet}\right) \otimes_{\mathbb{Z} / 2 \mathbb{Z}} Q_{R_{a}, U_{a}, f_{a}, i_{a}}\right]\right|_{R_{a} \cap R_{b}} \longrightarrow } \\
& {\left.\left[i_{b}^{*}\left(\mathcal{P} \mathcal{V}_{U_{b}, f_{b}}^{\bullet}\right) \otimes_{\mathbb{Z} / 2 \mathbb{Z}} Q_{R_{b}, U_{b}, f_{b}, i_{b}}\right]\right|_{R_{a} \cap R_{b}} \in \operatorname{Perv}\left(R_{a} \cap R_{b}\right), }
\end{aligned}
$$

satisfying $\alpha_{a a}=$ id for all $a \in A$ and

$$
\left.\left.\alpha_{b c}\right|_{R_{a} \cap R_{b} \cap R_{c}} \circ \alpha_{a b}\right|_{R_{a} \cap R_{b} \cap R_{c}}=\left.\alpha_{a c}\right|_{R_{a} \cap R_{b} \cap R_{c}} \quad \text { for all } a, b, c \in A \text {. }
$$

Fix $a, b \in A$. By applying Theorem 6.3 to the critical charts $\left(R_{a}, U_{a}, f_{a}, i_{a}\right)$, $\left(R_{b}, U_{b}, f_{b}, i_{b}\right)$ at each $x \in R_{a} \cap R_{b}$, we can choose an indexing set $D_{a b}$ and for each $d \in D_{a b}$ subcharts $\left(R_{a}^{\prime d}, U_{a}^{\prime d}, f_{a}^{\prime d}, i_{a}^{\prime d}\right) \subseteq\left(R_{a}, U_{a}, f_{a}, i_{a}\right),\left(R_{b}^{\prime d}, U_{b}^{\prime d}, f_{b}^{\prime d}, i_{b}^{\prime d}\right) \subseteq$ $\left(R_{b}, U_{b}, f_{b}, i_{b}\right)$, a critical chart $\left(S^{d}, V^{d}, g^{d}, j^{d}\right)$ on $(X, s)$, and embeddings $\Phi^{d}$ : $\left(R_{a}^{\prime d}, U_{a}^{\prime d}, f_{a}^{\prime d}, i_{a}^{\prime d}\right) \hookrightarrow\left(S^{d}, V^{d}, g^{d}, j^{d}\right), \Psi^{d}:\left(R_{b}^{\prime d}, U_{b}^{\prime d}, f_{b}^{\prime d}, i_{b}^{\prime d}\right) \hookrightarrow\left(S^{d}, V^{d}, g^{d}, j^{d}\right)$, such that $\left\{R_{a}^{\prime d} \cap R_{b}^{\prime d}: d \in D_{a b}\right\}$ is a Zariski open cover of $R_{a} \cap R_{b}$.

For each $d \in D_{a b}$, define an isomorphism

$$
\begin{gathered}
\alpha_{a b}^{d}:\left.\left[i_{a}^{*}\left(\mathcal{P} \mathcal{V}_{U_{a}, f_{a}}^{\bullet}\right) \otimes_{\mathbb{Z} / 2 \mathbb{Z}} Q_{R_{a}, U_{a}, f_{a}, i_{a}}\right]\right|_{R_{a}^{\prime d} \cap R_{b}^{\prime d}} \longrightarrow \\
{\left.\left[i_{b}^{*}\left(\mathcal{P} \mathcal{V}_{U_{b}, f_{b}}^{\bullet}\right) \otimes_{\mathbb{Z} / 2 \mathbb{Z}} Q_{R_{b}, U_{b}, f_{b}, i_{b}}\right]\right|_{R_{a}^{\prime d} \cap R_{b}^{\prime d}}}
\end{gathered}
$$

by the commutative diagram

$$
\begin{aligned}
& {\left[\left.\left.i_{a}^{*}\left(\mathcal{P} \mathcal{V}_{U_{a}, f_{a}}^{\bullet}\right) \otimes_{\mathbb{Z} / 2 \mathbb{Z}} \longrightarrow j^{d}\right|_{R_{a}^{\prime d} \cap R_{b}^{\prime d}} ^{*}\left(\mathcal{P} \mathcal{V}_{V^{d}, g^{d}}^{\bullet}\right) \otimes_{\mathbb{Z} / 2 \mathbb{Z}} i_{a}\right|_{R_{a}^{\prime d} \cap R_{b}^{\prime d}} ^{*}\left(P_{\Phi^{d}}\right)\right.} \\
& \left.Q_{R_{a}, U_{a}, f_{a}, i_{a}}\right]\left.\left.\right|_{R_{a}^{\prime d} \cap R_{b}^{\prime d}} \longrightarrow \otimes_{\left.i_{a}\right|_{R_{a}^{\prime} \cap} ^{*} \cap R_{b}^{\prime \prime}}\left(\Theta_{\Phi^{d}}\right) \quad Q_{\mathbb{Z} / 2 \mathbb{Z}} Q_{R_{a}, U_{a}, f_{a}, i_{a}}\right|_{R_{a}^{\prime d} \cap R_{b}^{\prime d}} \\
& \begin{array}{cc} 
& \otimes \operatorname{id}_{Q_{R_{a}, U_{a}, f_{a}, i_{a}}} \\
\alpha_{a b}^{d} & {\left.\left[\left(j^{d}\right)^{*}\left(\mathcal{P} \mathcal{V}_{V^{d}, g^{d}}^{\bullet}\right) \otimes_{\mathbb{Z} / 2 \mathbb{Z}} Q_{S^{d}, V^{d}, g^{d}, j^{d}}\right]\right|_{R_{a}^{\prime d} \cap R_{b}^{\prime d}} ^{-1} \downarrow}
\end{array}
\end{aligned}
$$

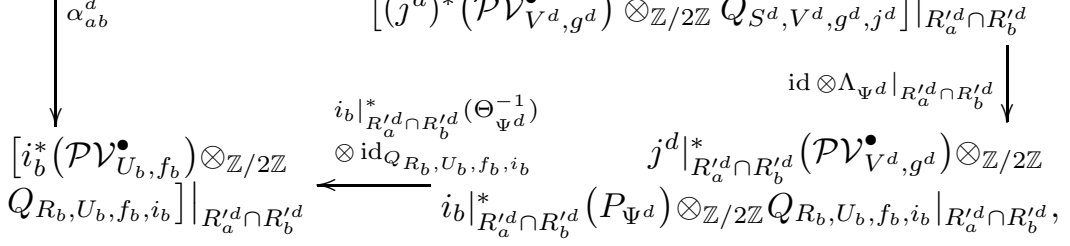

where $\Theta_{\Phi^{d}}, \Theta_{\Psi^{d}}$ are as in Theorem [5.4 and $\Lambda_{\Phi^{d}}, \Lambda_{\Psi^{d}}$ as in (6.9).

We claim that for all $d, e \in D_{a b}$ we have

$$
\left.\alpha_{a b}^{d}\right|_{R_{a}^{\prime d} \cap R_{b}^{\prime d} \cap R_{a}^{\prime e} \cap R_{b}^{\prime e}}=\left.\alpha_{a b}^{e}\right|_{R_{a}^{\prime d} \cap R_{b}^{\prime d} \cap R_{a}^{\prime e} \cap R_{b}^{\prime e}} .
$$


To see this, let $x \in R_{a}^{\prime d} \cap R_{b}^{\prime d} \cap R_{a}^{\prime e} \cap R_{b}^{\prime e}$, and apply Theorem 6.3 to the critical charts $\left(S^{d}, V^{d}, g^{d}, j^{d}\right),\left(S^{e}, V^{e}, g^{e}, j^{e}\right)$ and point $x \in S^{d} \cap S^{e}$. This gives subcharts $\left(S^{\prime d}, V^{\prime d}, g^{\prime d}, j^{\prime d}\right) \subseteq\left(S^{d}, V^{d}, g^{d}, j^{d}\right),\left(S^{\prime e}, V^{\prime e}, g^{\prime e}, j^{\prime e}\right) \subseteq\left(S^{e}, V^{e}, g^{e}, j^{e}\right)$ with $x \in S^{\prime d} \cap S^{\prime e}$, a critical chart $(T, W, h, k)$ on $(X, s)$, and embeddings $\Omega:\left(S^{\prime d}, V^{\prime d}, g^{\prime d}, j^{\prime d}\right) \hookrightarrow(T, W, h, k), \Upsilon:\left(S^{\prime e}, V^{\prime e}, g^{\prime e}, j^{\prime e}\right) \hookrightarrow(T, W, h, k)$.

Set $R^{d e}=R_{a}^{\prime d} \cap R_{b}^{\prime d} \cap R_{a}^{\prime e} \cap R_{b}^{\prime e} \cap S^{\prime d} \cap S^{\prime e}$, and consider the diagram:

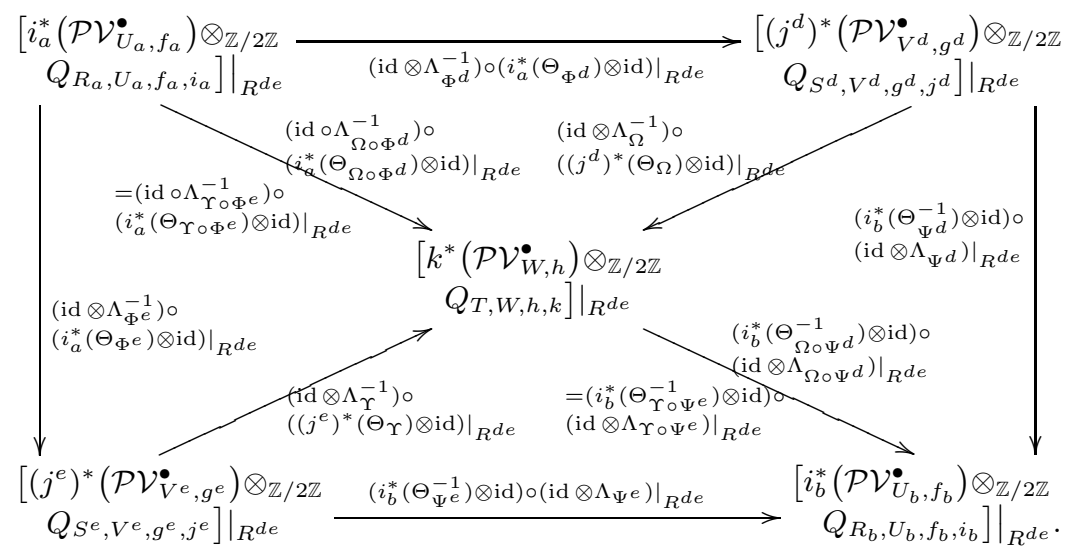

Here we have given two expressions for the top left diagonal morphism in (6.19). To see these are equal, set $R_{a}^{\prime d e}=R_{a}^{\prime d} \cap R_{a}^{\prime e} \cap S^{\prime d} \cap S^{\prime e}, U_{a}^{\prime d e}=$ $\left(\Phi^{d}\right)^{-1}\left(V^{\prime d}\right) \cap\left(\Phi^{e}\right)^{-1}\left(V^{\prime e}\right), f_{a}^{\prime d e}=\left.f_{a}\right|_{U_{a}^{\prime d e}}$, and $i_{a}^{\prime d e}=\left.i_{a}\right|_{R_{a}^{\prime d e}}$. Then $\left(R_{a}^{\prime d e}, U_{a}^{\prime d e}\right.$, $\left.f_{a}^{\prime d e}, i_{a}^{\prime d e}\right) \subseteq\left(R_{a}, U_{a}, f_{a}, i_{a}\right)$ is a subchart and

$$
\left.\Omega \circ \Phi^{d}\right|_{U_{a}^{\prime d e}},\left.\Upsilon \circ \Phi^{e}\right|_{U_{a}^{\prime d e}}:\left(R_{a}^{\prime d e}, U_{a}^{\prime d e}, f_{a}^{\prime d e}, i_{a}^{\prime d e}\right) \longrightarrow(T, W, h, k)
$$

are embeddings. As $\Omega \circ \Phi^{d} \circ i_{a}^{\prime d e}=\left.k\right|_{R_{a}^{\prime d e}}=\Upsilon \circ \Phi^{e} \circ i_{a}^{\prime d e}$, Theorem 5.4(b) gives $\left.\Theta_{\Omega \circ \Phi^{d}}\right|_{i_{a}\left(R_{a}^{\prime d e}\right)}=\left.\Theta_{\Upsilon \circ \Phi^{e}}\right|_{i_{a}\left(R_{a}^{\prime d e}\right)}$, so that $\left.i_{a}\right|_{R^{d e}} ^{*}\left(\Theta_{\Omega \circ \Phi^{d}}\right)=\left.i_{a}\right|_{R^{d e}} ^{*}\left(\Theta_{\Upsilon \circ \Phi^{e}}\right)$ as $R^{d e} \subseteq R_{a}^{\prime d e}$. Also $\Lambda_{\Omega_{\circ \Phi^{d}}}=\Lambda_{\Upsilon \circ \Phi^{e}}$ as these are defined in Theorem 6.9(ii) using $J_{\Omega \circ \Phi^{d}}, J_{\Upsilon \circ \Phi^{e}}$, which are equal by Lemma 5.3 So the two expressions are equal, and similarly for the bottom right diagonal morphism.

The upper triangle in (6.19) commutes because (5.16) gives

$$
\left.\left(\mathrm{id} \otimes \Xi_{\Omega, \Phi^{d}}\right) \circ \Theta_{\Omega \circ \Phi^{d}}\right|_{i_{a}\left(R^{d e}\right)}=\left.\left(\left.\Phi^{d}\right|_{i_{a}\left(R^{d e}\right)} ^{*}\left(\Theta_{\Omega}\right) \otimes \mathrm{id}\right) \circ \Theta_{\Phi^{d}}\right|_{i_{a}\left(R^{d e}\right)},
$$

and the definitions of $\Xi_{\Omega, \Phi^{d}}$ in (5.11) and $\Lambda_{\Omega}, \Lambda_{\Phi^{d}}, \Lambda_{\Omega \circ \Phi^{d}}$ in (6.9) imply that

$$
\begin{aligned}
& \left.\left(\left.i_{a}\right|_{R^{d e}} ^{*}\left(\Xi_{\Omega, \Phi^{d}}\right) \otimes \mathrm{id}\right) \circ \Lambda_{\Omega \circ \Phi^{d}}\right|_{R^{d e}}=\left.\left(\mathrm{id} \otimes \Lambda_{\Phi^{d}}\right) \circ \Lambda_{\Omega}\right|_{R^{d e}}: \\
& \left.\left.\left.\left.Q_{T, W, h, k}\right|_{R^{d e}} \longrightarrow j^{d}\right|_{R^{d e}} ^{*}\left(P_{\Omega}\right) \otimes_{\mathbb{Z} / 2 \mathbb{Z}} i_{a}\right|_{R^{d e}} ^{*}\left(P_{\Phi^{d}}\right) \otimes_{\mathbb{Z} / 2 \mathbb{Z}} Q_{R_{a}, U_{a}, f_{a}, i_{a}}\right|_{R^{d e}} .
\end{aligned}
$$

Similarly, the other three triangles in (6.19) commute, so (6.19) commutes.

By (6.17), the two routes round the outside of (6.19) are $\left.\alpha_{a b}^{d}\right|_{R^{d e}}$ and $\left.\alpha_{a b}^{e}\right|_{R^{d e}}$, which are equal as (6.19) commutes. As we can cover $R_{a}^{\prime d} \cap R_{b}^{\prime d} \cap R_{a}^{\prime e} \cap R_{b}^{\prime e}$ by such Zariski open $R^{d e}$, equation (6.18) follows. Therefore by the Zariski open 
cover version of Theorem 2.7)(i), there is a unique isomorphism $\alpha_{a b}$ in (6.15) such that $\left.\alpha_{a b}\right|_{R_{a}^{\prime d} \cap R_{b}^{\prime d}}=\alpha_{a b}^{d}$ for all $d \in D_{a b}$.

If $D_{a b}, R_{a}^{\prime d}, \ldots, \Phi^{d}, \Psi^{d}$ are used to define $\alpha_{a b}$ and $\tilde{D}_{a b}, \tilde{R}_{a}^{\prime d}, \ldots, \tilde{\Phi}^{d}, \tilde{\Psi}^{d}$ are alternative choices yielding $\tilde{\alpha}_{a b}$, then by our usual argument using $D_{a b} \amalg \tilde{D}_{a b}$ and both sets of data we see that $\alpha_{a b}=\tilde{\alpha}_{a b}$, so $\alpha_{a b}$ is independent of choices.

Because the $\Theta_{\Phi^{d}}, \Theta_{\Psi^{d}}$ used to define $\alpha_{a b}$ are compatible with Verdier duality and monodromy by (5.14) $-(5.15)$, and the $\Lambda_{\Phi^{d}}, \Lambda_{\Psi^{d}}$ affect only the principal $\mathbb{Z} / 2 \mathbb{Z}$-bundles rather than the perverse sheaves, we can show $\alpha_{a b}$ is compatible with Verdier duality and monodromy, in that the following commute:

$$
\begin{aligned}
& {\left[i _ { a } ^ { * } ( \mathcal { P } \mathcal { V } _ { U _ { a } , f _ { a } } ^ { \bullet } ) \otimes _ { \mathbb { Z } / 2 \mathbb { Z } } \longrightarrow \left[i_{b}^{*}\left(\mathcal{P} \mathcal{V}_{U_{b}, f_{b}}^{\bullet}\right) \otimes_{\mathbb{Z} / 2 \mathbb{Z}}\right.\right.} \\
& \left.\left.Q_{R_{a}, U_{a}, f_{a}, i_{a}}\right]\left.\right|_{R_{a} \cap R_{b}} \quad Q_{R_{b}, U_{b}, f_{b}, i_{b}}\right]\left.\right|_{R_{a} \cap R_{b}} \\
& \downarrow i_{a}^{*}\left(\sigma_{U_{a}, f_{a}}\right) \otimes \operatorname{id}_{Q_{R_{a}, U_{a}, f_{a}, i_{a}}}\left|R_{a} \cap R_{b} \quad i_{b}^{*}\left(\sigma_{U_{b}, f_{b}}\right) \otimes \operatorname{id}_{Q_{R_{b}}, U_{b}, f_{b}, i_{b}}\right| R_{a} \cap R_{b} \mid \\
& {\left[i _ { a } ^ { * } ( \mathbb { D } _ { \operatorname { C r i t } ( f _ { a } ) } ( \mathcal { P } \mathcal { V } _ { U _ { a } , f _ { a } } ^ { \bullet } ) ) \quad \left[i_{b}^{*}\left(\mathbb{D}_{\operatorname{Crit}\left(f_{b}\right)}\left(\mathcal{P} \mathcal{V}_{U_{b}, f_{b}}^{\bullet}\right)\right)\right.\right.} \\
& \left.\left.\left.\left.\otimes_{\mathbb{Z} / 2 \mathbb{Z}} Q_{R_{a}, U_{a}, f_{a}, i_{a}}\right]\left.\right|_{R_{a} \cap R_{b}}\right) \quad \otimes_{\mathbb{Z} / 2 \mathbb{Z}} Q_{R_{b}, U_{b}, f_{b}, i_{b}}\right]\left.\right|_{R_{a} \cap R_{b}}\right) \\
& \cong \mathbb{D}_{R_{a} \cap R_{b}}\left(\left[i _ { a } ^ { * } ( \mathcal { P } \mathcal { V } _ { U _ { a } , f _ { a } } ^ { \bullet } ) \quad \mathbb { D } _ { R _ { a } \cap R _ { b } } ( \alpha _ { a b } ) \cong \mathbb { D } _ { R _ { a } \cap R _ { b } } \left(\left[i_{b}^{*}\left(\mathcal{P} \mathcal{V}_{U_{b}, f_{b}}^{\bullet}\right)\right.\right.\right.\right.
\end{aligned}
$$

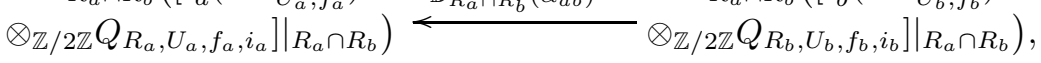

$$
\begin{aligned}
& {\left[i _ { a } ^ { * } ( \mathcal { P } \mathcal { V } _ { U _ { a } , f _ { a } } ^ { \bullet } ) \otimes _ { \mathbb { Z } / 2 \mathbb { Z } } \longrightarrow \left[i_{b}^{*}\left(\mathcal{P} \mathcal{V}_{U_{b}, f_{b}}^{\bullet}\right) \otimes_{\mathbb{Z} / 2 \mathbb{Z}}\right.\right.} \\
& \left.\left.Q_{R_{a}, U_{a}, f_{a}, i_{a}}\right]\left.\right|_{R_{a} \cap R_{b}} \quad Q_{R_{b}, U_{b}, f_{b}, i_{b}}\right]\left.\right|_{R_{a} \cap R_{b}} \\
& i_{a}^{*}\left(\tau_{U_{a}, f_{a}}\right) \otimes \mathrm{id}_{Q_{R_{a}, U_{a}, f_{a}, i_{a}}}\left|R_{a} \cap R_{b} \quad \quad i_{b}^{*}\left(\tau_{U_{b}, f_{b}}\right) \otimes \operatorname{id}_{Q_{R_{b}, U_{b}, f_{b}, i_{b}}}\right|_{R_{a} \cap R_{b}} \downarrow \\
& {\left[i _ { a } ^ { * } ( \mathcal { P } \mathcal { V } _ { U _ { a } , f _ { a } } ^ { \bullet } ) \otimes _ { \mathbb { Z } / 2 \mathbb { Z } } \quad \left[i_{b}^{*}\left(\mathcal{P V}_{U_{b}, f_{b}}^{\bullet}\right) \otimes_{\mathbb{Z} / 2 \mathbb{Z}}\right.\right.} \\
& \left.\left.Q_{R_{a}, U_{a}, f_{a}, i_{a}}\right]\left.\right|_{R_{a} \cap R_{b}} \longrightarrow Q_{R_{b}, U_{b}, f_{b}, i_{b}}\right]\left.\right|_{R_{a} \cap R_{b}} \text {. }
\end{aligned}
$$

When $a=b$ we can take $\Psi^{d}=\Phi^{d}$, so (6.17) gives $\alpha_{a a}^{d}=\mathrm{id}$, and $\alpha_{a a}=\mathrm{id}$.

To prove (6.16), let $a, b, c \in A$, and $x \in R_{a} \cap R_{b} \cap R_{c}$. Applying Theorem 6.3 twice and composing the embeddings, we can construct subcharts $\left(R_{a}^{\prime}, U_{a}^{\prime}, f_{a}^{\prime}, i_{a}^{\prime}\right) \subseteq\left(R_{a}, U_{a}, f_{a}, i_{a}\right),\left(R_{b}^{\prime}, U_{b}^{\prime}, f_{b}^{\prime}, i_{b}^{\prime}\right) \subseteq\left(R_{b}, U_{b}, f_{b}, i_{b}\right),\left(R_{c}^{\prime}, U_{c}^{\prime}, f_{c}^{\prime}, i_{c}^{\prime}\right)$ $\subseteq\left(R_{c}, U_{c}, f_{c}, i_{c}\right)$ with $x \in R_{a}^{\prime} \cap R_{b}^{\prime} \cap R_{c}^{\prime}$, a critical chart $(S, V, g, j)$ on $(X, s)$, and embeddings $\Phi:\left(R_{a}^{\prime}, U_{a}^{\prime}, f_{a}^{\prime}, i_{a}^{\prime}\right) \hookrightarrow(S, V, g, j), \Psi:\left(R_{b}^{\prime}, U_{b}^{\prime}, f_{b}^{\prime}, i_{b}^{\prime}\right) \hookrightarrow(S, V, g, j)$, $\Upsilon:\left(R_{c}^{\prime}, U_{c}^{\prime}, f_{c}^{\prime}, i_{c}^{\prime}\right) \hookrightarrow(S, V, g, j)$. Then the construction of $\alpha_{a b}$ above yields

$$
\begin{aligned}
&\left.\alpha_{a b}\right|_{R_{a}^{\prime} \cap R_{b}^{\prime} \cap R_{c}^{\prime}}=\left.\left(\left(i_{b}^{*}\left(\Theta_{\Psi}^{-1}\right) \otimes \mathrm{id}\right) \circ\left(\mathrm{id} \otimes \Lambda_{\Psi}\right) \circ\left(\mathrm{id} \otimes \Lambda_{\Phi}^{-1}\right) \circ\left(i_{a}^{*}\left(\Theta_{\Phi}\right) \otimes \mathrm{id}\right)\right)\right|_{R_{a}^{\prime} \cap R_{b}^{\prime} \cap R_{c}^{\prime}}, \\
&\left.\alpha_{b c}\right|_{R_{a}^{\prime} \cap R_{b}^{\prime} \cap R_{c}^{\prime}}=\left.\left(\left(i_{c}^{*}\left(\Theta_{\Upsilon}^{-1}\right) \otimes \mathrm{id}\right) \circ\left(\mathrm{id} \otimes \Lambda_{\Upsilon}\right) \circ\left(\mathrm{id} \otimes \Lambda_{\Psi}^{-1}\right) \circ\left(i_{b}^{*}\left(\Theta_{\Psi}\right) \otimes \mathrm{id}\right)\right)\right|_{R_{a}^{\prime} \cap R_{b}^{\prime} \cap R_{c}^{\prime}}, \\
&\left.\alpha_{a c}\right|_{R_{a}^{\prime} \cap R_{b}^{\prime} \cap R_{c}^{\prime}}=\left.\left(\left(i_{c}^{*}\left(\Theta_{\Upsilon}^{-1}\right) \otimes \mathrm{id}\right) \circ\left(\mathrm{id} \otimes \Lambda_{\Upsilon}\right) \circ\left(\mathrm{id} \otimes \Lambda_{\Phi}^{-1}\right) \circ\left(i_{a}^{*}\left(\Theta_{\Phi}\right) \otimes \mathrm{id}\right)\right)\right|_{R_{a}^{\prime} \cap R_{b}^{\prime} \cap R_{c}^{\prime}},
\end{aligned}
$$

so that $\left.\left.\alpha_{b c}\right|_{R_{a}^{\prime} \cap R_{b}^{\prime} \cap R_{c}^{\prime}} \circ \alpha_{a b}\right|_{R_{a}^{\prime} \cap R_{b}^{\prime} \cap R_{c}^{\prime}}=\left.\alpha_{a c}\right|_{R_{a}^{\prime} \cap R_{b}^{\prime} \cap R_{c}^{\prime}}$. As we can cover $R_{a} \cap R_{b} \cap$ $R_{c}$ by such Zariski open $R_{a}^{\prime} \cap R_{b}^{\prime} \cap R_{c}^{\prime}$, equation (6.16) follows by Theorem 2.7 (i).

The Zariski open cover version of Theorem 2.7(ii) now implies that there exists $P_{X, s}^{\bullet}$ in $\operatorname{Perv}(X)$, unique up to canonical isomorphism, with isomorphisms

$$
\omega_{R_{a}, U_{a}, f_{a}, i_{a}}:\left.P_{X, s}^{\bullet}\right|_{R_{a}} \longrightarrow i_{a}^{*}\left(\mathcal{P} \mathcal{V}_{U_{a}, f_{a}}^{\bullet}\right) \otimes_{\mathbb{Z} / 2 \mathbb{Z}} Q_{R_{a}, U_{a}, f_{a}, i_{a}}
$$


as in (6.6) for each $a \in A$, with $\alpha_{a b} \circ \omega_{R_{a}, U_{a}, f_{a}, i_{a}}\left|R_{a} \cap R_{b}=\omega_{R_{b}, U_{b}, f_{b}, i_{b}}\right|_{R_{a} \cap R_{b}}$ for all $a, b \in A$. Also, (6.7) -6.8) with $\left(R_{a}, U_{a}, f_{a}, i_{a}\right)$ in place of $(R, U, f, i)$ define isomorphisms $\left.\Sigma_{X, s}\right|_{R_{a}},\left.\mathrm{~T}_{X, s}\right|_{R_{a}}$ for each $a \in A$. Equations (6.20)-6.21) imply that the prescribed values for $\left.\Sigma_{X, s}\right|_{R_{a}},\left.\mathrm{~T}_{X, s}\right|_{R_{a}}$ and $\left.\Sigma_{X, s}\right|_{R_{b}},\left.\mathrm{~T}_{X, s}\right|_{R_{b}}$ agree when restricted to $R_{a} \cap R_{b}$ for all $a, b \in A$. Hence, Theorem 2.7(i) gives unique isomorphisms $\Sigma_{X, s}, \mathrm{~T}_{X, s}$ in (6.5) such that (6.7) commute with $\left(R_{a}, U_{a}, f_{a}, i_{a}\right)$ in place of $(R, U, f, i)$ for all $a \in A$.

Suppose $\left\{\left(R_{a}, U_{a}, f_{a}, i_{a}\right): a \in A\right\}$ and $\left\{\left(\tilde{R}_{a}, \tilde{U}_{a}, \tilde{f}_{a}, \tilde{\imath}_{a}\right): a \in \tilde{A}\right\}$ are alternative choices above, yielding $P_{X, s}^{\bullet}, \Sigma_{X, s}, \mathrm{~T}_{X, s}$ and $\tilde{P}_{X, s}^{\bullet}, \tilde{\Sigma}_{X, s}, \tilde{\mathrm{T}}_{X, s}$. Then applying the same construction to the family $\left\{\left(R_{a}, U_{a}, f_{a}, i_{a}\right): a \in A\right\} \amalg$ $\left\{\left(\tilde{R}_{a}, \tilde{U}_{a}, \tilde{f}_{a}, \tilde{\imath}_{a}\right): a \in \tilde{A}\right\}$ to get $\hat{P}_{X, s}^{\bullet}$, we have canonical isomorphisms $P_{X, s}^{\bullet} \cong$ $\hat{P}_{X, s}^{\bullet} \cong \tilde{P}_{X, s}^{\bullet}$, which identify $\Sigma_{X, s}, \mathrm{~T}_{X, s}$ with $\tilde{\Sigma}_{X, s}, \tilde{\mathrm{T}}_{X, s}$. Thus $P_{X, s}^{\bullet}, \Sigma_{X, s}, \mathrm{~T}_{X, s}$ are independent of choices up to canonical isomorphism.

Now fix $\left\{\left(R_{a}, U_{a}, f_{a}, i_{a}\right): a \in A\right\}, P_{X, s}^{\bullet}, \Sigma_{X, s}, \mathrm{~T}_{X, s}$ and $\omega_{R_{a}, U_{a}, f_{a}, i_{a}}$ for $a \in A$ above for the rest of the proof. Suppose $(R, U, f, i)$ is a critical chart on $(X, s)$. Running the construction above with the family $\left\{\left(R_{a}, U_{a}, f_{a}, i_{a}\right)\right.$ : $a \in A\} \amalg\{(R, U, f, i)\}$, we can suppose it yields the same (not just isomorphic) $P_{X, s}^{\bullet}, \Sigma_{X, s}, \mathrm{~T}_{X, s}$ and $\omega_{R_{a}, U_{a}, f_{a}, i_{a}}$, but it also yields a unique $\omega_{R, U, f, i}$ in (6.6) which makes (6.7)-(6.8) commute. This proves Theorem 6.9)(i).

Let $\Phi:(R, U, f, i) \hookrightarrow(S, V, g, j)$ be an embedding of critical charts on $(X, s)$. The definition of $\Lambda_{\Phi}$ in Theorem 6.9)(ii) is immediate. Run the construction above using the family $\left\{\left(R_{a}, U_{a}, f_{a}, i_{a}\right): a \in A\right\} \amalg\{(R, U, f, i),(S, V, g, j)\}$, and follow the definition of $\alpha_{a b}$ with $(R, U, f, i),(S, V, g, j)$ in place of $\left(R_{a}, U_{a}, f_{a}, i_{a}\right)$, $\left(R_{b}, U_{b}, f_{b}, i_{b}\right)$. We can take $D_{a b}=\{d\},\left(R_{a}^{\prime d}, U_{a}^{\prime d}, f_{a}^{\prime d}, i_{a}^{\prime d}\right)=(R, U, f, i),\left(R_{b}^{\prime d}\right.$, $\left.U_{b}^{\prime d}, f_{b}^{\prime d}, i_{b}^{\prime d}\right)=\left(S^{d}, V^{d}, g^{d}, j^{d}\right)=(S, V, g, j), \Phi^{d}=\Phi$ and $\Psi^{d}=\operatorname{id}_{V}$. Then (6.17) gives $\alpha_{a b}=\alpha_{a b}^{d}=\left(\mathrm{id} \otimes \Lambda_{\Phi}^{-1}\right) \circ\left(i^{*}\left(\Theta_{\Phi}\right) \otimes \mathrm{id}\right)$. Thus, $\left.\alpha_{a b} \circ \omega_{R_{a}, U_{a}, f_{a}, i_{a}}\right|_{R_{a} \cap R_{b}}=$ $\left.\omega_{R_{b}, U_{b}, f_{b}, i_{b}}\right|_{R_{a} \cap R_{b}}$ implies that (6.10) commutes, proving Theorem 6.9)(ii).

\section{4 $\mathscr{D}$-modules and mixed Hodge modules}

Once again, the proof of Theorem 6.9 carries over to our other contexts in 2.6 2.10 using the general framework of 2.5 now also making use of the Stack Property (x) for objects. For the case of mixed Hodge modules, we use Theorem 2.21 (ii) to glue the $i_{a}^{*}\left(\mathcal{H} \mathcal{V}_{U_{a}, f_{a}}^{\bullet}\right) \otimes_{\mathbb{Z} / 2 \mathbb{Z}} Q_{R_{a}, U_{a}, f_{a}, i_{a}}$ on $R_{a} \subseteq X$ for $a \in A$ with their natural strong polarizations (2.25), which are preserved by the isomorphisms $\alpha_{a b}$ in $₫ 6.3$ on overlaps $R_{a} \cap R_{b}$.

\section{A Compatibility results, by Jörg Schürmann}

In the main body of the paper, when comparing results for mixed Hodge modules to those involving perverse sheaves, we rely on the compatibility between duality and Thom-Sebastiani type isomorphisms of perverse sheaves and mixed Hodge modules. These compatibility statements cannot easily be read off from the existing literature, so we provide proofs here. 
Proposition A.1. If $X$ is a $\mathbb{C}$-scheme and $f: X \rightarrow \mathbb{C}$ is regular, then Massey's natural isomorphisms from [37. quoted as Theorem 2.11(iv) coincide with the image under the realization functor of Saito's analogous isomorphisms [45] between functors on mixed Hodge modules. There is also an analogous compatibility result for $X$ a complex analytic space equipped with an analytic function $f$.

Proof. Massey's construction in 37] of the duality isomorphisms uses the definition of the vanishing cycle functor in terms of the local cohomology of suitable real half-spaces, compare also [50. Using their notation, the compatibility comes down to compatibility of the diagram

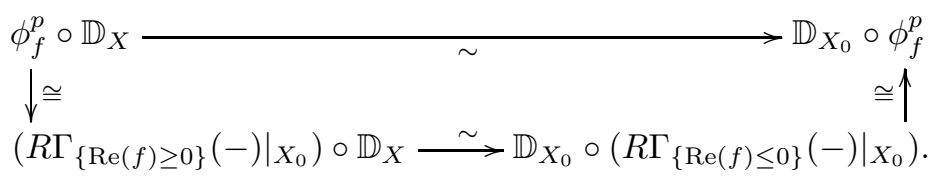

Here the upper, respectively lower horizontal isomorphisms are the ones of Saito, respectively Massey, and the vertical isomorphisms follow for example from [50, Lem. 1.3.2, p. 69]. Saito deduces his duality isomorphism in [45, Lem. 5.2.4, p. 965] from a pairing on nearby cycles induced by a pairing $F \otimes G \rightarrow$ $a_{X}^{!} A$ for $F, G \in D_{c}^{b}(X)$, with $a_{X}: X \rightarrow$ pt the constant map and $A \subset \mathbb{C}$ a coefficient field. But Massey's duality isomorphism can be also be induced from such a pairing fitting into a commutative diagram, with $L_{0}=\{\operatorname{Re}(f)=0\}$ and $j:\{\operatorname{Re}(f)=0, f \neq 0\} \rightarrow L_{0}$ the open inclusion:

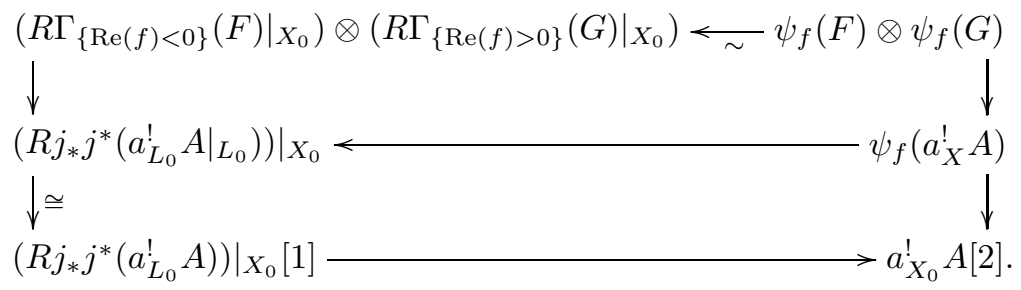

Here the isomorphism $j^{*}\left(\left.a_{X}^{!} A\right|_{L_{0}}\right) \cong j^{*}\left(a_{L_{0}}^{!} A\right)[1]$ comes from the fact that $\operatorname{Re}(f)$ has no critical points (in a stratified sense) in $X \backslash X_{0}$, locally near $X_{0}$. But then the commutativity of (A.2) implies by [45, Lem. 5.2.4, p. 965] the commutativity of (A.1), concluding the proof.

A similar compatibility question arises for the Thom-Sebastiani isomorphism. Here the precise statement is the following.

Proposition A.2. Let $f_{i}: Y_{i} \rightarrow \mathbb{C}$ be regular functions on smooth $\mathbb{C}$-schemes, for $i=1,2$. Let $f=f_{1} \boxplus f_{2}: Y_{1} \times Y_{2} \rightarrow \mathbb{C}$ be as in Theorem 2.13. Then the isomorphism (2.8) of Massey [35, 50. coincides with the image under the realization functor of Saito's analogous isomorphism (2.26) of [49] for mixed Hodge modules. 
Proof. The Thom-Sebastiani isomorphism (2.26) is constructed by Saito [49, Th. 2.6] based on the Verdier specialization [53]. First, let $f: Y \rightarrow \mathbb{C}$ be a regular function, with $X=f^{-1}(0)$ of codimension one, so that the normal cone $C_{X} Y=X \times \mathbb{C}$ becomes a trivial line bundle with $f^{\prime}: C_{X} Y \rightarrow \mathbb{C}$ given by the projection. Let $p: D_{X} Y \rightarrow \mathbb{C}$ be the deformation to the normal cone with $C_{X} Y=p^{-1}(0) \subset D_{X} Y$, with $q: D_{X} Y \rightarrow Y$ the natural map. Then $f^{\prime}$ extends to a function $g: D_{X} Y \rightarrow \mathbb{C}$, with $g=f / s$ on $p \neq 0=Y \times \mathbb{C}$ for $s$ the usual coordinate on $\mathbb{C}$. For $F \in D_{c}^{b}(Y)$, we get a commutative diagram

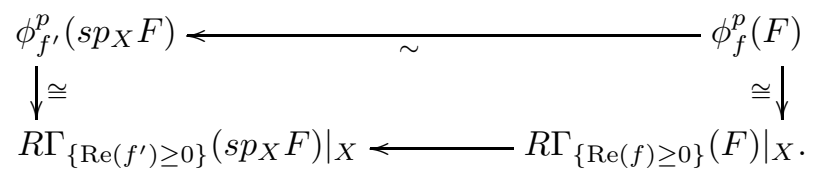

Here the monodromical sheaf complex $s p_{X} F \in D_{c}^{b}\left(C_{X} Y\right)_{\text {mon }}$ is the Verdier specialization of $F$ as in [49,53]. The upper horizontal isomorphism is the one of [49, Lem. 2.2], whereas the vertical isomorphisms are those of [50, Lem. 1.3.2, p. 69]. The lower horizontal map is defined by the natural base change morphism

$$
\left.\left.\left.R \Gamma_{\left\{\operatorname{Re}\left(f^{\prime}\right) \geq 0\right\}}\left(s p_{X} F\right)\right|_{X} \longleftarrow \psi_{p}\left(R \Gamma_{\{\operatorname{Re}(g) \geq 0\}}\left(q^{*} F\right)\right)\right|_{X} \cong R \Gamma_{\{\operatorname{Re}(f) \geq 0\}}(F)\right|_{X},
$$

where the last isomorphism follows as in [50, Lem. 1.3.3, p. 70-71].

Consider now the situation in the proposition, with

$$
f=f_{1} \boxplus f_{2}: Y=Y_{1} \times Y_{2} \rightarrow \mathbb{C},
$$

also $X_{i}=f_{i}^{-1}(0)$ and $X=f^{-1}(0)$; finally let $\mu_{f_{i}}=R \Gamma_{\left\{\operatorname{Re}\left(f_{i}\right) \geq 0\right\}}$ to shorten the notation. Let

$$
\pi: C_{X_{1}} Y_{1} \times C_{X_{2}}\left(Y_{2}\right)=\left(X_{1} \times X_{2}\right) \times \mathbb{C}^{2} \rightarrow\left(X_{1} \times X_{2}\right) \times \mathbb{C} \subset C_{X}(Y)
$$

be the map induced by addition in the fibres. Then, for $F_{i} \in D_{c}^{b}\left(Y_{i}\right)$, one gets a commutative diagram

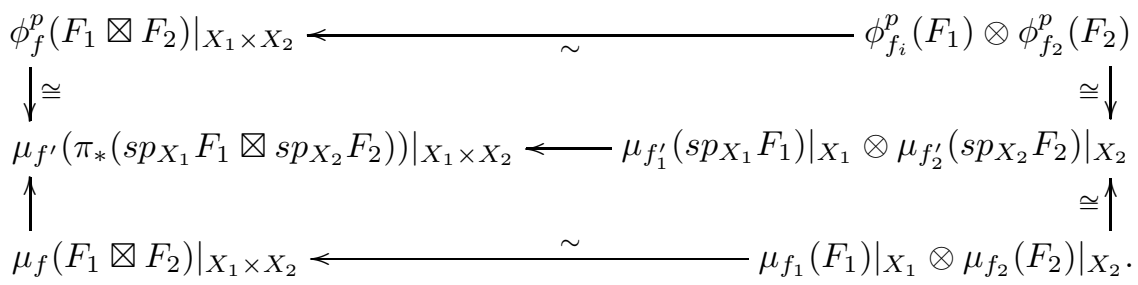

The upper horizontal and left vertical isomorphisms form the Thom-Sebastiani isomorphism (2.26) of 49, whereas the lower horizontal isomorphism is the Thom-Sebastiani isomorphism (2.8) of 35,50. This concludes the proof. 


\section{References}

[1] V.I. Arnold, S.M. Gusein-Zade and A.N. Varchenko, Singularities of differentiable maps, Volume 1, Monographs in Math. 82, Birkhäuser, 1985.

[2] K. Behrend, Donaldson-Thomas type invariants via microlocal geometry, Ann. of Math. 170 (2009), 1307-1338. math.AG/0507523.

[3] K. Behrend, J. Bryan and B. Szendrői, Motivic degree zero DonaldsonThomas invariants, Invent. Math. 192 (2013), 111-160. arXiv:0909.5088

[4] K. Behrend and B. Fantechi, Gerstenhaber and Batalin-Vilkovisky structures on Lagrangian intersections, pages 1-47 in Algebra, arithmetic, and geometry, Progr. Math. 269, Birkhäuser, Boston, MA, 2009.

[5] A.A. Beilinson, J. Bernstein and P. Deligne, Faisceaux pervers, Astérisque $100,1982$.

[6] O. Ben-Bassat, C. Brav, V. Bussi, and D. Joyce, A 'Darboux Theorem' for shifted symplectic structures on derived Artin stacks, with applications, Geometry and Topology 19 (2015), 1287-1359. arXiv:1312.0090.

[7] J.-E. Björk, Analytic $\mathscr{D}$-modules and applications, Kluwer, Dordrecht, 1993.

[8] A. Borel et al., Algebraic $\mathscr{D}$-modules, Perspectives in Mathematics 2, Academic Press, 1987.

[9] C. Brav, V. Bussi and D. Joyce, A Darboux theorem for derived schemes with shifted symplectic structure, arXiv:1305.6302, 2013.

[10] V. Bussi, Categorification of Lagrangian intersections on complex symplectic manifolds using perverse sheaves of vanishing cycles, arXiv:1404.1329, 2014 .

[11] V. Bussi, D. Joyce and S. Meinhardt, On motivic vanishing cycles of critical loci, arXiv:1305.6428, 2013.

[12] S.C. Coutinho, A primer of algebraic $\mathscr{D}$-modules, L.M.S. Student texts 33, Cambridge University Press, 1995.

[13] A. D'Agnolo and P. Schapira, Quantization of complex Lagrangian submanifolds, Advances in Math. 213 (2007), 358-379. math.AG/0506064

[14] A. Dimca, Sheaves in Topology, Universitext, Springer-Verlag, Berlin, 2004.

[15] A. Dimca and B. Szendröi, The Milnor fibre of the Pfaffian and the Hilbert scheme of four points on $\mathbb{C}^{3}$, Math. Res. Lett. 16 (2009), 1037-1055. arXiv:0904.2419. 
[16] S.K. Donaldson and R.P. Thomas, Gauge Theory in Higher Dimensions, Chapter 3 in S.A. Huggett, L.J. Mason, K.P. Tod, S.T. Tsou and N.M.J. Woodhouse, editors, The Geometric Universe, Oxford University Press, Oxford, 1998.

[17] E. Freitag and R. Kiehl, Etale cohomology and the Weil Conjecture, Ergeb. der Math. und ihrer Grenzgebiete 13, Springer-Verlag, 1988.

[18] K. Fukaya, Y.-G. Oh, H. Ohta and K. Ono, Lagrangian intersection Floer theory - anomaly and obstruction, Parts I \& II. AMS/IP Studies in Advanced Mathematics, 46.1 \& 46.2, A.M.S./International Press, 2009.

[19] T. Gaffney and H. Hauser, Characterizing singularities of varieties and of mappings, Invent. math. 81 (1985), 427-447.

[20] G. Guibert, F. Loeser and M. Merle, Iterated vanishing cycles, convolution, and a motivic analogue of a conjecture of Steenbrink, Duke Math. J. 132 (2006), 409-457.

[21] R. Hotta, T. Tanisaki and K. Takeuchi, $\mathscr{D}$-modules, perverse sheaves, and representation theory, Progr. Math. 236, Birkhäuser, Boston, MA, 2008.

[22] D. Huybrechts and R.P. Thomas, Deformation-obstruction theory for complexes via Atiyah and Kodaira-Spencer classes, Math. Ann. 346 (2010), 545-569. arXiv:0805.3527.

[23] D. Joyce, A classical model for derived critical loci, to appear in Journal of Differential Geometry, 2015. arXiv:1304.4508.

[24] D. Joyce and Y. Song, A theory of generalized Donaldson-Thomas invariants, Mem. Amer. Math. Soc. 217 (2012), no. 1020. arXiv:0810.5645.

[25] M. Kashiwara, The Riemann-Hilbert problem for holonomic systems, Publ. RIMS 20 (1984), 319-365.

[26] M. Kashiwara, $\mathscr{D}$-modules and microlocal calculus, Trans. Math. Mono. 217, A.M.S., 2003.

[27] M. Kashiwara and P. Schapira, Sheaves on manifolds, Grundlehren der Math. Wiss. 292, Springer-Verlag, Berlin, 1990.

[28] M. Kashiwara and P. Schapira, Constructibility and duality for simple modules on symplectic manifolds, Amer. J. Math. 130 (2008), 207-237. math.QA/0512047.

[29] M. Kashiwara and P. Schapira, Deformation quantization modules, Astérisque 345, 2012.

[30] R. Kiehl and R. Weissauer, Weil Conjectures, perverse sheaves and l'adic Fourier transform, Springer-Verlag, 2001. 
[31] Y.-H. Kiem and J. Li, Categorification of Donaldson-Thomas invariants via perverse sheaves, arXiv:1212.6444, 2012.

[32] M. Kontsevich and Y. Soibelman, Stability structures, motivic DonaldsonThomas invariants and cluster transformations, arXiv:0811.2435, 2008.

[33] M. Kontsevich and Y. Soibelman, Cohomological Hall algebra, exponential Hodge structures and motivic Donaldson-Thomas invariants, Commun. Number Theory Phys. 5 (2011), 231-352. arXiv:1006.2706.

[34] P. Maisonobe and Z. Mebkhout, Le théorème de comparaison pour les cycles évanescents, pages 311-389 in Éléments de la théorie des systèmes différentiels géométriques, Sémin. Congr., 8, Soc. Math. France, Paris, 2004.

[35] D. Massey, The Sebastiani-Thom isomorphism in the derived category, Compositio Math. 125 (2001), 353-362. math.AG/9908101.

[36] D. Massey, Notes on perverse sheaves and vanishing cycles, math.AG/9908107, 1999.

[37] D. Massey, Natural commuting of vanishing cycles and the Verdier dual, arXiv:0908.2799, 2009.

[38] J. Mather and S.S. Yau, Classification of isolated hypersurface singularities by their moduli algebra, Invent. Math. 69 (1982), 243-251.

[39] L. Maxim, M. Saito and J. Schürmann, Symmetric products of mixed Hodge modules, J. Math. Pures Appl. 96 (2011), 462-483.

[40] D. Nadler, Microlocal branes are constructible sheaves, Selecta Math. 15 (2009), 563-619. math.SG/0612399.

[41] D. Nadler and E. Zaslow, Constructible sheaves and the Fukaya category, J. Amer. Math. Soc. 22 (2009), 233-286. math.SG/0604379.

[42] T. Pantev, B. Toën, M. Vaquié and G. Vezzosi, Shifted symplectic structures, Publ. Math. I.H.E.S. 117 (2013), 271-328. arXiv:1111.3209.

[43] K. Rietsch, An introduction to perverse sheaves, pages 391-429 in V. Dlab and C.M. Ringel, editors, Representations of finite dimensional algebras and related topics in Lie theory and geometry, Fields Inst. Commun. 40, A.M.S., Providence, RI, 2004. math.RT/0307349

[44] C. Sabbah, On a twisted de Rham complex, II, arXiv:1012.3818, 2010.

[45] M. Saito, Modules de Hodge polarisables, Publ. RIMS 24 (1988), 849-995.

[46] M. Saito, Duality for vanishing cycle functors, Publ. RIMS 25 (1989), 889921. 
[47] M. Saito, Mixed Hodge Modules, Publ. RIMS 26 (1990), 221-333.

[48] M. Saito, D-modules on analytic spaces, Publ. RIMS 27 (1991), 291-332.

[49] M. Saito, Thom-Sebastiani Theorem for Hodge Modules, preprint, 2010.

[50] J. Schürmann, Topology of singular spaces and constructible sheaves, Monografie Matematyczne 63, Birkhäuser, Basel, 2003.

[51] B. Szendröi, Nekrasov's partition function and refined Donaldson-Thomas theory: the rank one case, SIGMA (2012) 088, 16pp. arXiv:1210.5181.

[52] R.P. Thomas, A holomorphic Casson invariant for Calabi-Yau 3-folds, and bundles on K3 fibrations, J. Diff. Geom. 54 (2000), 367-438. math.AG/9806111.

[53] J.-L. Verdier, Spécialization de faisceaux et monodromie modérée, Astérisque 101-102 (1983), 332-364.

AdDress for Christopher Brav:

Faculty of Mathematics, Higher School of Economics, 7 Vavilova Str., Moscow, Russia

E-MAIL: chris.i.brav@gmail.com.

ADDRESS FOR VITTORIA BUSSI:

ICTP, Strada Costiera 11, Trieste, Italy

E-MAIL: vbussi@ictp.it.

AdDRESS FOR DOMINIC JOYCE AND BALÁZS SZENDRŐI:

The Mathematical Institute, Woodstock Road, Oxford, UK

E-MAILS: joyce@maths.ox.ac.uk, szendroi@maths.ox.ac.uk.

ADDRESS FOR JÖRG SCHÜRMANN:

Mathematische Institut, Universität Münster, Einsteinstrasse 62, 48149 Münster, Germany.

E-MAIL: jschuerm@math.uni-muenster.de. 